Florida International University FIU Digital Commons

\title{
A Case-Only Genome-wide Association Study of Gender- and Age-specific Risk Markers for Childhood Leukemia
}

Sandeep Kumar Singh

ssing023@fiu.edu

DOI: $10.25148 /$ etd.FI15032162

Follow this and additional works at: https://digitalcommons.fiu.edu/etd

Part of the Environmental Public Health Commons

\section{Recommended Citation}

Singh, Sandeep Kumar, "A Case-Only Genome-wide Association Study of Gender- and Age-specific Risk Markers for Childhood Leukemia" (2015). FIU Electronic Theses and Dissertations. 1832.

https://digitalcommons.fiu.edu/etd/1832 


\section{FLORIDA INTERNATIONAL UNIVERSITY}

Miami, Florida

\section{A CASE-ONLY GENOME-WIDE ASSOCIATION STUDY OF GENDER- AND AGE-SPECIFIC RISK MARKERS FOR CHILDHOOD LEUKEMIA}

A dissertation submitted in partial fulfillment of

the requirements for the degree of

DOCTOR OF PHILOSOPHY

in

PUBLIC HEALTH

by

Sandeep Kumar Singh

2015 
To: Interim Dean Mark Williams

Robert Stempel College of Public Health \& Social Work

This dissertation, written by Sandeep Kumar Singh, and entitled A Case-only GenomeWide Association Study of Gender- and Age- Specific Risk Markers for Childhood Leukemia, having been approved in respect to style and intellectual content, is referred to you for judgment.

We have read this dissertation and recommend that it be approved.

Boubakari Ibrahimou

$\begin{array}{r}\hline \text { Helen Tempest } \\ \hline \text { Wehmet T. Dorak, Co-Major Professor } \\ \hline \text { Stanislaw F. Wnuk, Co-Major Professor }\end{array}$

Date of Defense: March 26, 2015

The dissertation of Sandeep Kumar Singh is approved.

Interim Dean Mark Williams Robert Stempel College of Public Health \& Social Work

Dean Lakshmi N. Reddi University Graduate School

Florida International University, 2015 
C Copyright 2015 by Sandeep Kumar Singh

All rights reserved. 


\section{DEDICATION}

I dedicate this dissertation to my entire family, especially to the three beautiful ladies of my life: my wife Anusha, my daughter Aanya, and my late grandmother Pannavati Singh. My parents never left my side and are very special. Without support and encouragement of my late grandfather, Bhagwati Prasad Singh, this dissertation was not possible. 


\section{ACKNOWLEDGMENTS}

I am using this opportunity to express my gratitude to everyone who supported me throughout my dissertation. I am thankful for their inspiring guidance, invaluable constructive criticism and friendly advice during the whole course work. I am sincerely grateful to them for sharing their truthful and illuminating views on a number of issues related to this dissertation.

I express my warm thanks to my major Professor, Dr. Stanislaw F. Wnuk and comajor Dr. Mehmet T. Dorak for all of their guidance and support and most important of all for believing in me. I would also like to thank the members of my dissertation committee, Dr. Wasim Maziak, Dr. Helen Tempest, and Dr. Boubakari Ibrahimou for their time and efforts in guiding my research. This dissertation had not been possible without guidance of Dr. Jacob L. McCauley, Dr Marcus E. Cooke, Dr. O. Dale Williams, Dr. Deodutta Roy and Dr. Tan Li.

I would also like to thank the Robert Stempel College of Public Health and Social Work, Department of Environmental and Occupational Health, and the Department of Epidemiology for supporting me through graduate assistantship. I am also grateful to FIU's University Graduate School for providing me the Dissertation Year Fellowship.

Last, but not least, I would like to thank all my friends for their support, especially Anshul Saxena, Abhishek Verma, Soumyadeep Mukharjee and Dominic Lomando for their constant help and support. 


\title{
ABSTRACT OF THE DISSERTATION \\ A CASE-ONLY GENOME-WIDE ASSOCIATION STUDY OF GENDER- AND AGE-SPECIFIC RISK MARKERS FOR CHILDHOOD LEUKEMIA
}

\author{
by \\ Sandeep Kumar Singh \\ Florida International University, 2015 \\ Miami, Florida

\begin{abstract}
Professor Stanislaw F. Wnuk, Co-Major Professor
Professor Mehmet T. Dorak, Co-Major Professor
\end{abstract}

Males and age group 1 to 5 years show a much higher risk for childhood acute lymphoblastic leukemia (ALL). We performed a case-only genome-wide association study (GWAS), using the Illumina Infinium HumanCoreExome Chip, to unmask genderand age-specific risk variants in 240 non-Hispanic white children with ALL recruited at Texas Children's Cancer Center, Houston, Texas. Besides statistically most significant results, we also considered results that yielded the highest effect sizes. Existing experimental data and bioinformatic predictions were used to complement results, and to examine the biological significance of statistical results.

Our study identified novel risk variants for childhood ALL. The SNP, rs4813720 (RASSF2), showed the statistically most significant gender-specific associations $(\mathrm{P}<2 \mathrm{x}$ $\left.10^{-6}\right)$. Likewise, rs 10505918 (SOX5) yielded the lowest $\mathrm{P}$ value $\left(\mathrm{P}<1 \times 10^{-5}\right)$ for agespecific associations, and also showed the statistically most significant association with age-at-onset $\left(\mathrm{P}<1 \times 10^{-4}\right)$. Two SNPs, rs12722042 and 12722039, from the HLA-DQA1 region yielded the highest effect sizes (odds ratio $(O R)=15.7 ; \mathrm{P}=0.002$ ) for gender- 
specific results, and the SNP, rs17109582 (OR = 12.5; $\mathrm{P}=0.006)$, showed the highest effect size for age-specific results. Sex chromosome variants did not appear to be involved in gender-specific associations.

The HLA-DQA1 SNPs belong to DQA1*01:07and confirmed previously reported male-specific association with DQA1*01:07. Twenty one of the SNPs identified as risk markers for gender- or age-specific associations were located in the transcription factor binding sites and 56 SNPs were non-synonymous variants, likely to alter protein function. Although bioinformatic analysis did not implicate a particular mechanism for gender- and age-specific associations, RASSF2 has an estrogen receptor-alpha binding site in its promoter. The unknown mechanisms may be due to lack of interest in gender- and agespecificity in associations. These results provide a foundation for further studies to examine the gender- and age-differential in childhood ALL risk. Following replication and mechanistic studies, risk factors for one gender or age group may have a potential to be used as biomarkers for targeted intervention for prevention and maybe also for treatment. 


\section{TABLE OF CONTENTS}

CHAPTER

PAGE

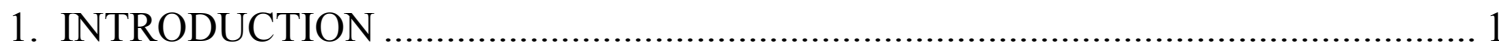

1.1. Descriptive epidemiology of childhood acute lymphoblastic leukemia ................. 1

1.2. Gender difference in childhood acute lymphoblastic leukemia.............................. 2

1.3. Age peak in childhood acute lymphoblastic leukemia ......................................... 3

1.4. Ethnic differences in childhood acute lymphoblastic leukemia ............................ 4

1.5. Risk factors contributing to childhood acute lymphoblastic leukemia .................... 4

1.6. Statement of the problem and public health significance .................................... 6

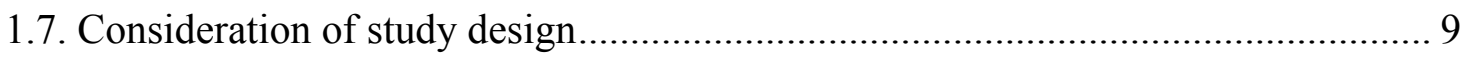

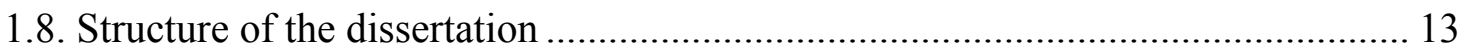

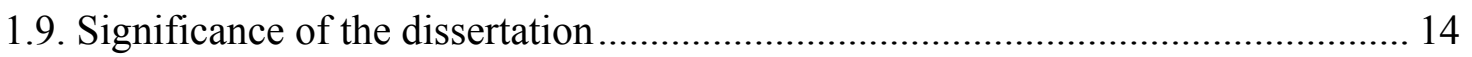

1.9.1. Better understanding of gender- and age-disparities in childhood acute

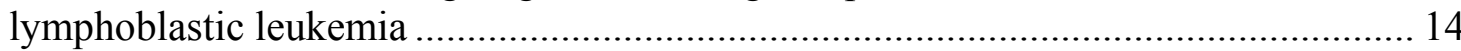

1.9.2. Guidance for new strategies for studying other complex diseases ................... 16

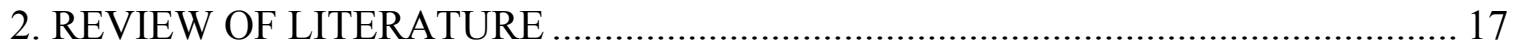

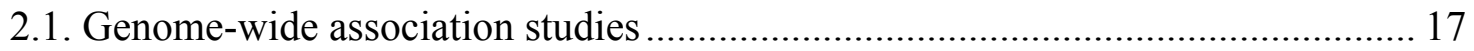

2.2. Gender disparity in cancer incidence .............................................................. 19

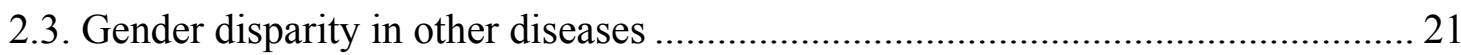

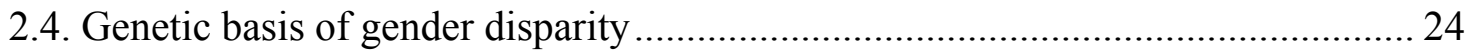

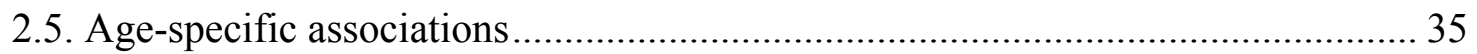

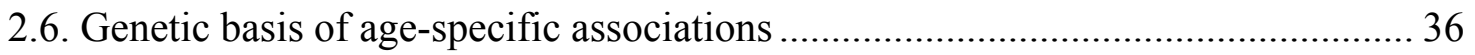

3. PROJECT AIMS AND HYPOTHESIS.............................................................. 41

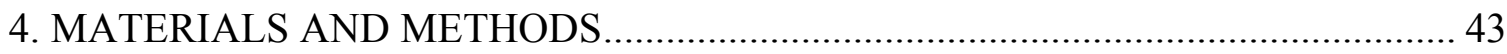

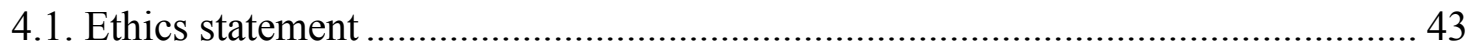

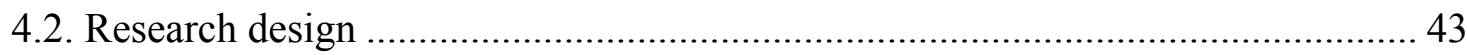

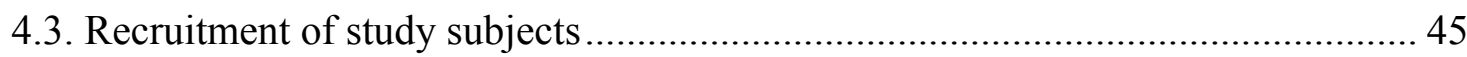

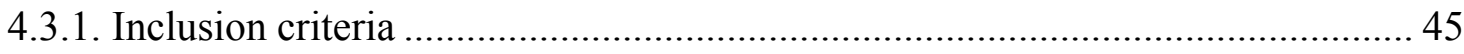

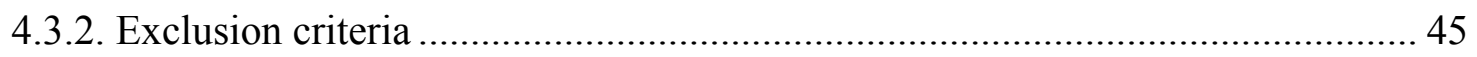

4.4. Statistical power calculation for statistical interaction when gender-specific associations differ in direction (qualitative interaction) ......................................... 46 
4.5. Statistical power calculation for statistical interaction when gender-specific associations differ in magnitude (quantitative interaction) ..................................... 47

4.6. Statistical power calculation for time-to-event analysis ..................................... 48

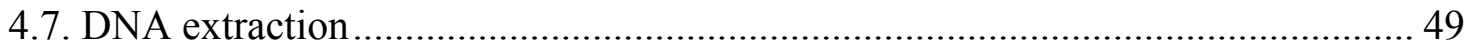

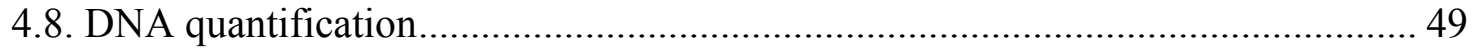

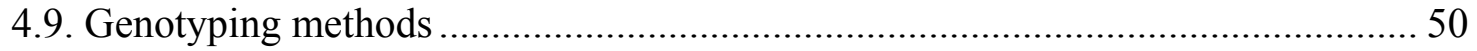

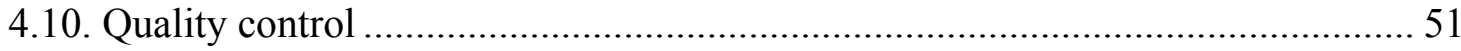

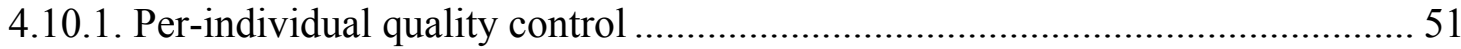

4.10.1.1. Identification of individuals with discordant gender information ................. 53

4.10.1.2. Identification of individuals with excessive missing rate or outlying

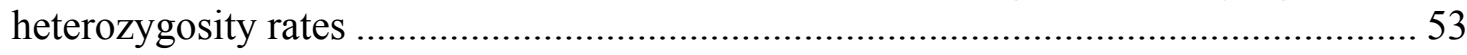

4.10.1.3. Identification of duplicated or related individuals .................................. 53

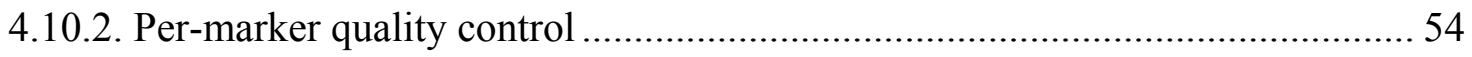

4.10.2.1. Identification of SNPs with an excessive missing genotyping rate .............. 54

4.10.2.2. Removal of markers with a very low minor allele frequency ....................... 54

4.10.2.3. Identification of SNPs with significantly different missing genotype rates

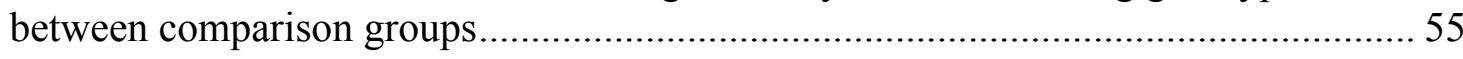

4.10.2.4. Identification of SNPs showing a significant deviation from Hardy-

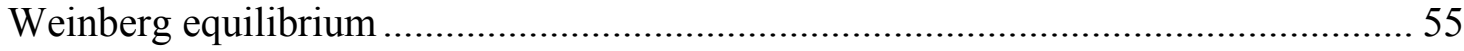

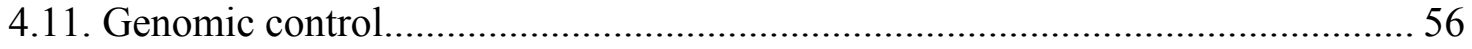

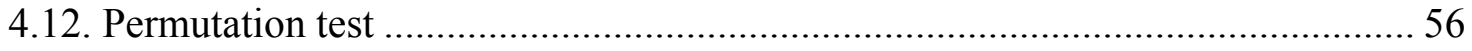

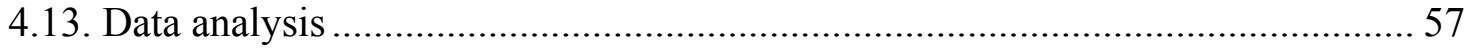

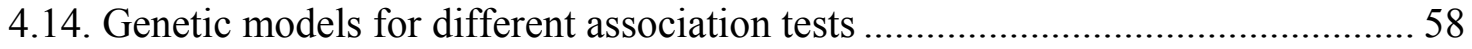

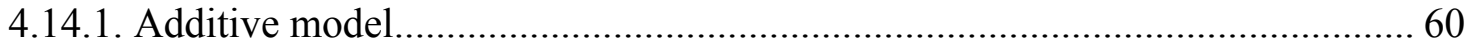

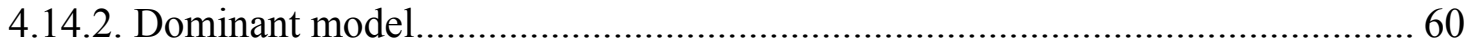

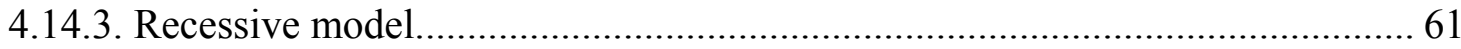

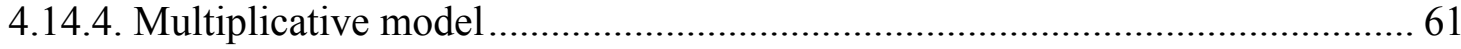

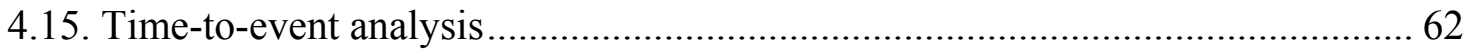

4.16. Bioinformatic and empirical screening of data for functional annotation of

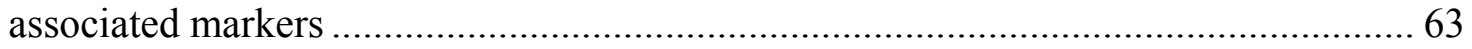

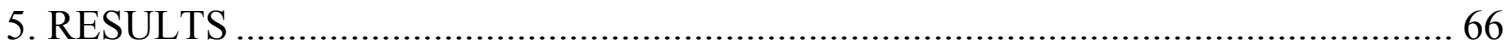

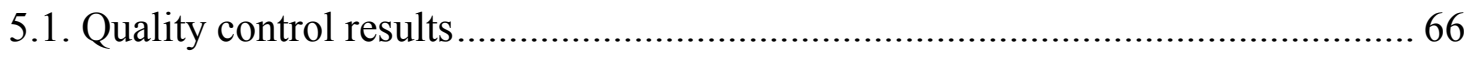

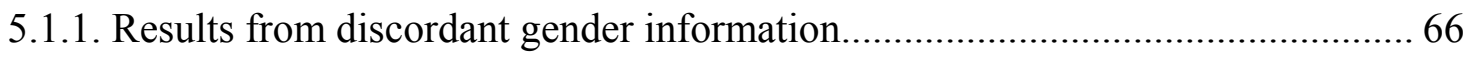

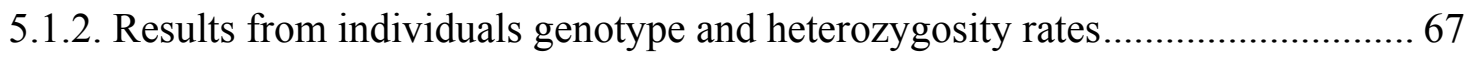




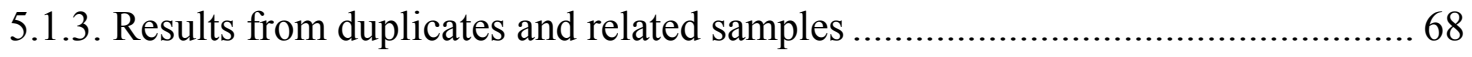

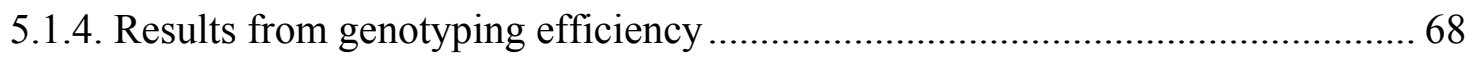

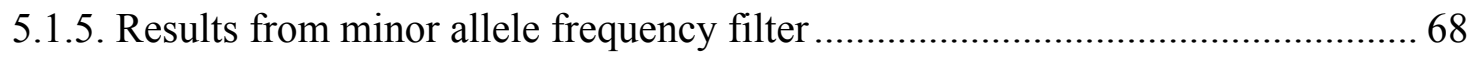

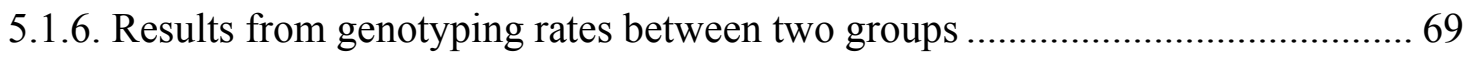

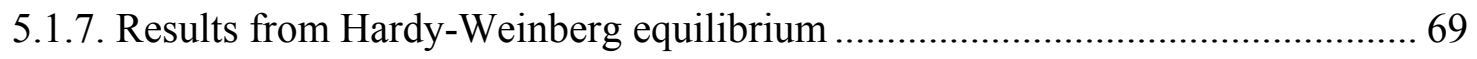

5.2. Characteristics of childhood ALL study subjects ............................................... 71

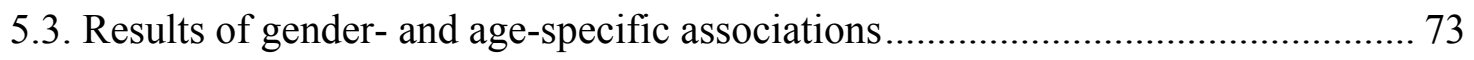

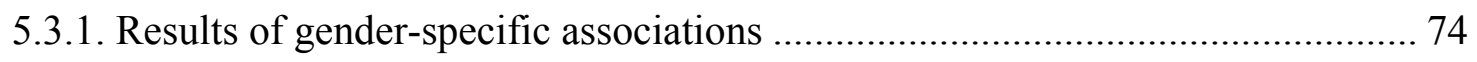

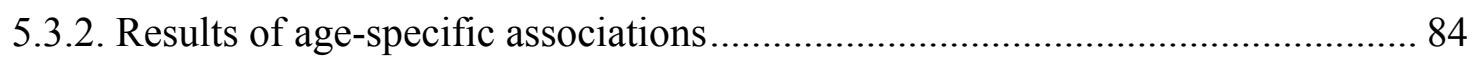

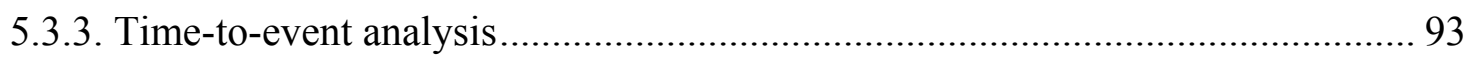

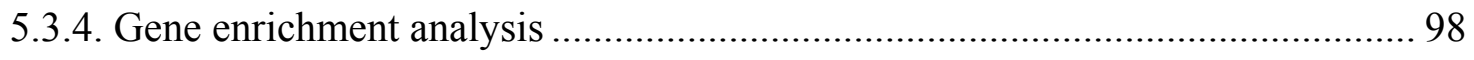

5.3.5. Previously identified gender- and age-specific associations .............................. 98

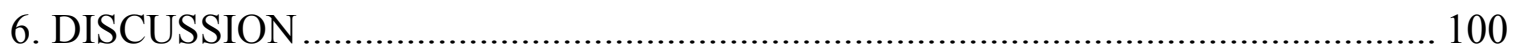

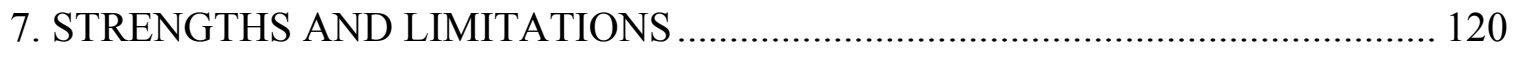

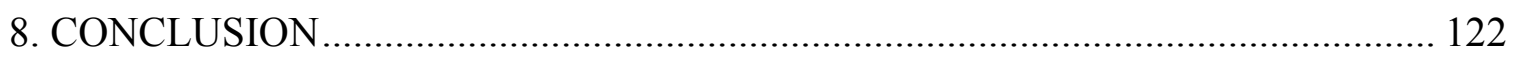

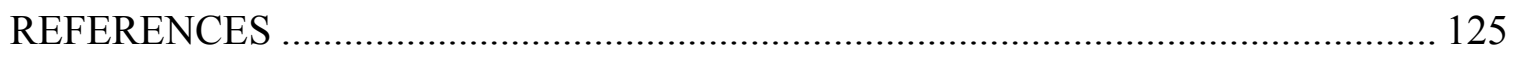

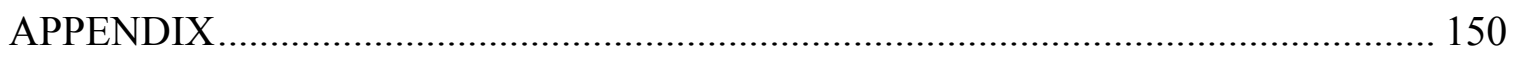

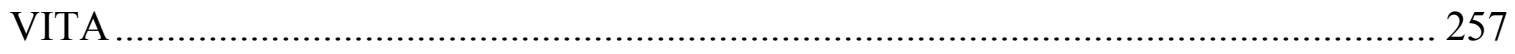




\section{LIST OF TABLES}

TABLE

PAGE

Table 1. Risk factors (known, suggestive or with limited evidence) associated with childhood acute lymphoblastic ...................................................................... 5

Table 2. Odds ratio calculation for association of disease with gender, by genotype ...... 10

Table 3. Odds ratio calculation for association of disease with gender and genotype,

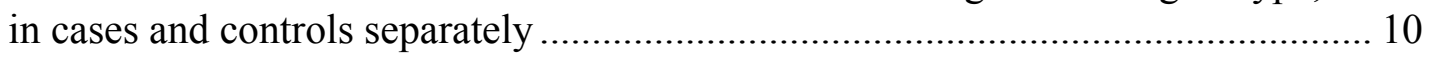

Table 4. Odds ratio for association of disease with gender and genotype, in case-only

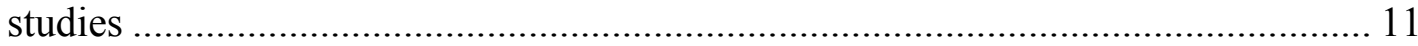

Table 5. Odds ratio for association of disease with age and genotype, in case-only studies 12

Table 6. SNPs associated with gender-specificity in childhood acute lymphoblastic

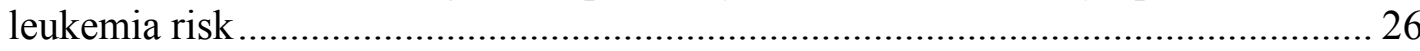

Table 7. SNPs associated with childhood acute lymphoblastic leukemia risk in previous genome-wide association studies with gender- and age-specificity

Table 8. Descriptive epidemiology of childhood acute lymphoblastic leukemia by age-at-diagnosis

Table 9. Statistical power calculations for the detection of interactions when associations differ in direction

Table 10. Statistical power calculations for association differing in magnitude 48

Table 11. Statistical power calculation for time-to-event analysis

Table 12. Summary of variables for gender-specific association 58

Table 13. Summary of variables for age-specific association 58

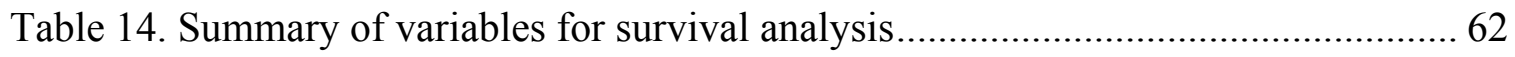

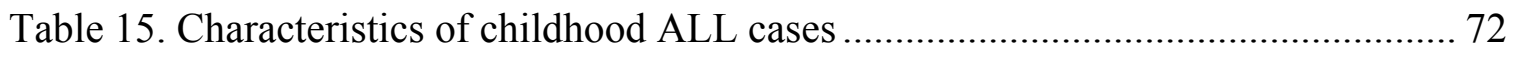

Table 16. Characteristics of male and female childhood acute lymphoblastic leukemia cases ..... 
Table 17. SNPs showed gender-specific associations in childhood ALL risk at the significance level $<1 \times 10^{-4}$

Table 18. SNPs showed gender-specific associations in childhood ALL risk with the highest effect sizes $(\mathrm{OR}>10 ; \mathrm{P}$ value $<0.05)$

Table 19. SNPs showed age-specific associations in childhood ALL risk at the significance level $<1 \times 10^{-4}$

Table 20. SNPs showed age-specific associations in childhood ALL risk with the highest effect sizes $(\mathrm{OR}>9$; $\mathrm{P}$ value $<0.05)$

Table 21. Time-to-event analysis of identified gender- and age-specific childhood ALL risk markers at the significance level $<1 \times 10^{-4}$. 


\section{LIST OF FIGURES}

FIGURE

PAGE

Figure 1. Childhood acute lymphoblastic leukemia incidence rates from 1975 to $2010 \ldots .2$

Figure 2. Gender differences in the incidence rate of childhood acute lymphoblastic leukemia 3

Figure 3. Age-specific incidence rates in childhood acute lymphoblastic leukemia by single year of age-at-diagnosis

Figure 4. United States childhood cancer survivors 7

Figure 5. Genetic variants may be either protective or risk markers for childhood ALL depending on the presence or absence of environmental risk factors.....

Figure 6. Difference in the direction of a gender-specific association for childhood ALL

Figure 7. Difference in the magnitude of a gender-specific association for childhood ALL 31

Figure 8. A flowchart explaining method section

Figure 9. Statistical power calculation for the detection of interactions using the case-only design 46

Figure 10. A flow chart of quality control steps used before the gender- and agespecific analysis

Figure 11. Common genetic risk models use in a genetic association study. 59

Figure 12. Quality control steps, and samples and markers exclusion 70

Figure 13. Q-Q plot for gender-specific association analysis for childhood ALL risk.

Figure 14. Manhattan plot for gender-specific association analysis for childhood acute lymphoblastic leukemia risk

Figure 15. Genome view of single nucleotide polymorphisms or associated genes in gender-specific associations based on $\mathrm{P}$ values 
Figure 16. Genome view of single nucleotide polymorphisms or associated genes in gender-specific associations based on effect size ............................................... 78

Figure 17. Q-Q plot for age-specific association analysis for childhood ALL risk......... 85

Figure 18. Genome view of single nucleotide polymorphisms or associated genes in

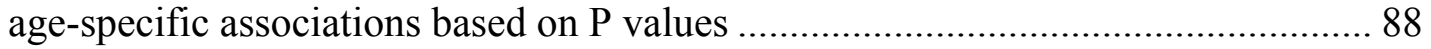

Figure 19. Genome view of single nucleotide polymorphisms or associated genes in

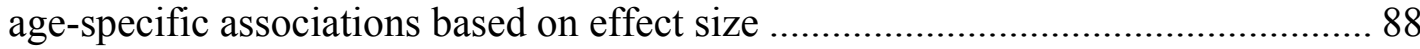

Figure 20. Manhattan plot for age-specific association analysis for childhood acute

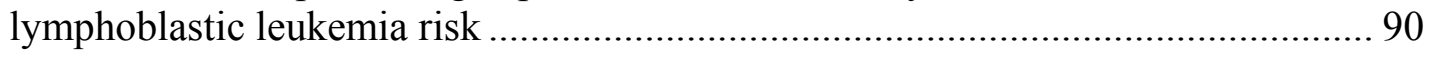

Figure 21. A flow chart explaining marker selection for time-to-event analysis ............ 93

Figure 22. Genome view of single nucleotide polymorphisms or associated genes with

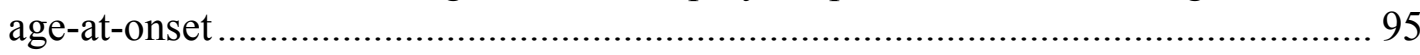

Figure 23. Kaplan-Meier plot estimates for age-at-diagnosis in childhood ALL with

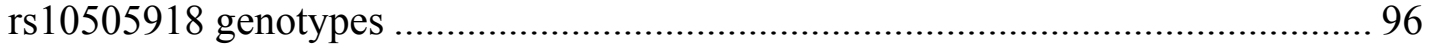

Figure 24. Kaplan-Meier plot estimates for age-at-diagnosis in childhood ALL with rs2949827 genotypes 


\section{ABBREVIATIONS AND ACRONYMS}

ALL

$\mathrm{AD}$

$\mathrm{BCM}$

CI

COSMIC

CLL

CML

CTD

CD

DNA

eQTL

FDA

F-SNP

GWAS

GC

HR

HWE

HCC

HLA

HIHG

IARC
Acute lymphoblastic leukemia

Alzheimer disease

Baylor College of Medicine

Confidence interval

Catalogue of Somatic Mutations in Cancers

Chronic lymphocytic leukemia

Chronic myeloid leukemia

Comparative Toxicogenomics Database

Crohn disease

Deoxyribonucleic acid

Expression quantitative trait locus

Food and Drug Administration

Functional Single Nucleotide Polymorphism

Genome-wide association study

Genomic control

Hazard ratio

Hardy-Weinberg equilibrium

Hepatocellular carcinoma

Human leukocyte antigens

Hussman Institute for Human Genomics

International Agency for Research on Cancer 
IBS

$\mathrm{IOM}$

IRB

IRR

LCL

MAF

NCI

$\mathrm{NIH}$

NHGRI

OR

PASS

PAH

QC

RA

RNA

SNP

SD

SEER

T1D

TXCCC
Identity by state

Institute of Medicine

Institutional Review Board

Incidence rate ratio

Lymphoblastoid cell line

Minor allele frequency

National Cancer Institute

National Institute Health

National Human Genome Research Institute

Odds ratio

Power analysis and sample size

Poly aromatic hydrocarbon

Quality control

Rheumatoid arthritis

Ribonucleic acid

Single nucleotide polymorphism

Standard deviation

Surveillance Epidemiology and End Result

Type 1 diabetes

Texas Children's Cancer Center 


\section{INTRODUCTION}

Acute lymphoblastic leukemia (ALL) is a cancer of white blood cells ${ }^{1}$. ALL is the most common form of childhood cancer in most parts of the world, except in Africa ${ }^{2}$.

Although ALL is relatively rare, its incidence rate has significantly increased $(0.8 \%$ per year) since 1975 in the United States ${ }^{3-5}$. The reasons for the increasing incidence rate are still unknown. Change in environmental factors may be responsible for some increase ${ }^{2}$. Unfortunately, childhood cancers are not public health priorities in most part of the world despite being a major cause of childhood death ${ }^{2-4}$. Unlike adult cancers, preventable risk factors for childhood cancers are mostly unknown ${ }^{3}$. Incomplete knowledge of childhood ALL etiology, unknown environmental risk factors, and known but understudied gender, age, and race/ethnicity differences signify the need for more research. For more than 95\% of cases, childhood ALL etiology is still unknown ${ }^{1-4}$, which makes formulating policies to reduce childhood ALL incidence impossible. Therefore, focused research to unmask the causes of childhood ALL is crucial.

\subsection{Descriptive epidemiology of childhood acute lymphoblastic leukemia}

Cancers are one of the major causes of death among children in the United States of America $^{2-4}$. Leukemia alone represents one-third of all childhood cancers of which $80 \%$ are ALL, with an annual incidence rate of 38 cases per $10^{6}$ children per year under age 14 , or 31 cases per $10^{6}$ children per year under age 19 in the United States ${ }^{2-5}$. Although treatment is now more successful, there is an increase in annual incidence rate by $0.6 \%$ per year from 2006 to 2010 in children aged 14 years and younger ${ }^{2-4}$. This trend has been consistent since 1975 (Figure 1). 


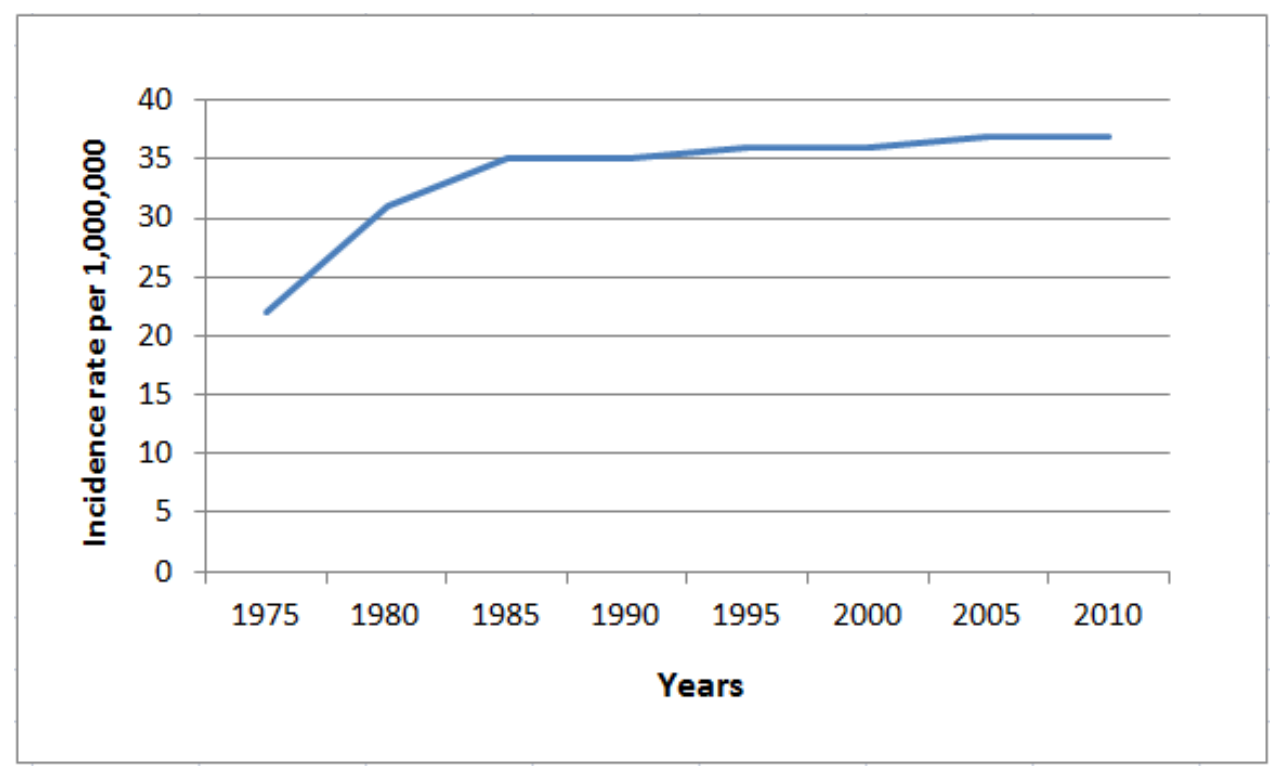

Figure 1. Childhood acute lymphoblastic leukemia incidence rates from 1975 to 2010

Age-adjusted Surveillance, Epidemiology, and End Results cancer-incidence rates, in childhood ALL, age 0-14 years. Incidence rates are per 1,000,000 per year and are age-adjusted to the United States standard population. $\mathrm{X}$-axis represents years and $\mathrm{Y}$-axis represents incidence rate per 1,000,000 per year.

Source: National Cancer Institute, Surveillance, Epidemiology, and End Results ${ }^{5}$

Furthermore, survivors of childhood ALL still suffer from late effects like relapse, social and behavioral issues, and secondary malignancies ${ }^{6-16}$. Thus, prevention is of paramount importance.

\subsection{Gender difference in childhood acute lymphoblastic leukemia}

Gender difference in cancers, whether it is childhood ${ }^{17-23}$ or adult ${ }^{4,24-26}$, specifically in hematological cancers ${ }^{27}$, is well known. Descriptive epidemiology consistently shows male predominance in childhood ALL (Figure 2) 2-4, 19, 21, 22, 26-30 . Males experience around 20\% higher incidence and worse prognosis than females in all ethnicities ${ }^{2-4,28}$. Moreover, the 5-year and 30-year survival rates are worse in male children with ALL, even after adjusting for age-at-diagnosis, race, year of diagnosis, and 
cell type ${ }^{31}$. Furthermore, relapse and secondary malignancies have been consistently reported more frequently in males ${ }^{31}$. Males are subject to more intense treatment protocols in childhood ALL as a result of having more severe disease ${ }^{23}$.

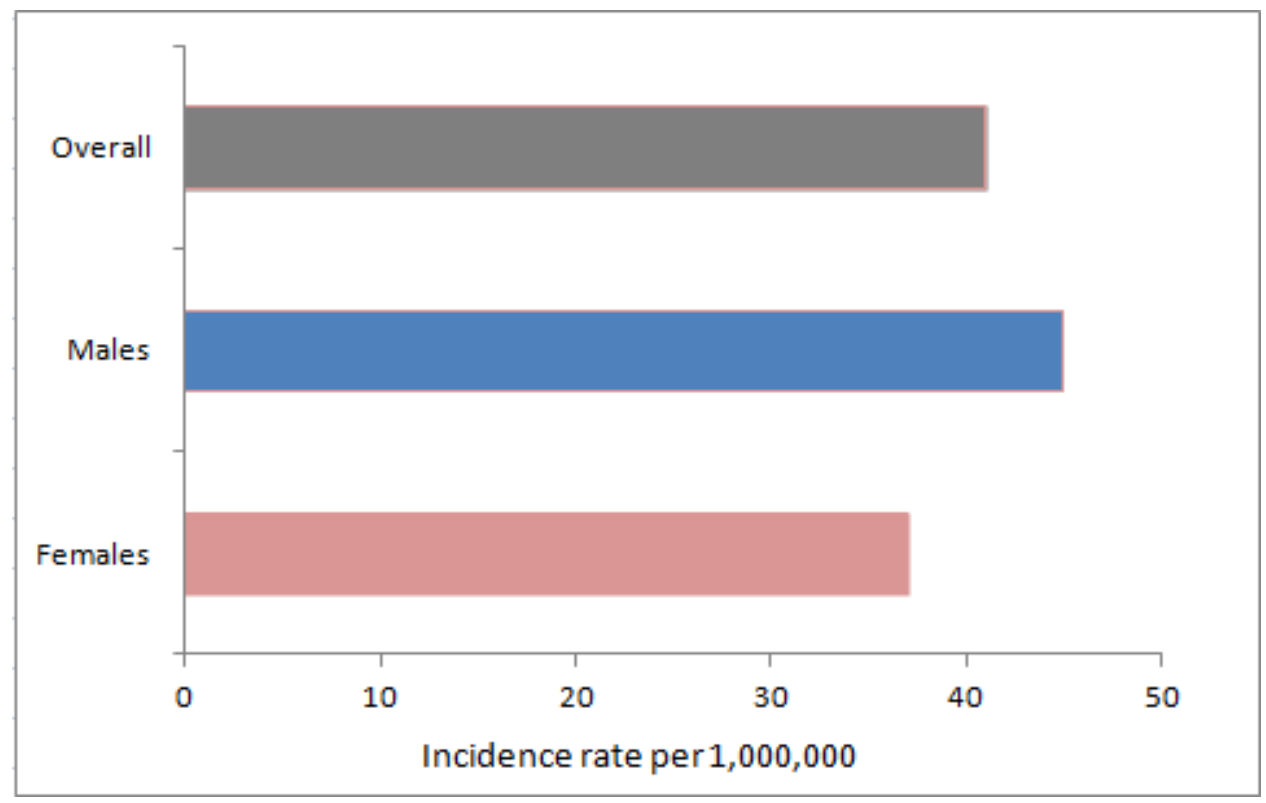

Figure 2. Gender differences in the incidence rate of childhood acute lymphoblastic leukemia Age-adjusted Surveillance, Epidemiology, and End Results cancer-incidence rates, 2006-2010, in childhood ALL, age 0-14 years. Incidence rates are per 1,000,000 per year and are age-adjusted to the United States standard population. Males show higher incidence rates. $\mathrm{X}$-axis represents incidence rate per 1,000,000 per year and $\mathrm{Y}$-axis represents both genders and overall incidence rates.

Source: National Cancer Institute, Surveillance, Epidemiology, and End Results ${ }^{5}$

\subsection{Age peak in childhood acute lymphoblastic leukemia}

Childhood ALL risk is highest among age group 1-5 years ${ }^{2,3,5}$ (Figure 3) with an annual incidence rate of 72 cases per $10^{6}$ children per year. Precursor B-cell leukemia is the most common type of childhood ALL in the age group 1-5 years. In contrast, infants $<1$ year of age suffer from a morphologically different type of leukemia that is more aggressive and shows a less favorable outcome ${ }^{1}$. 


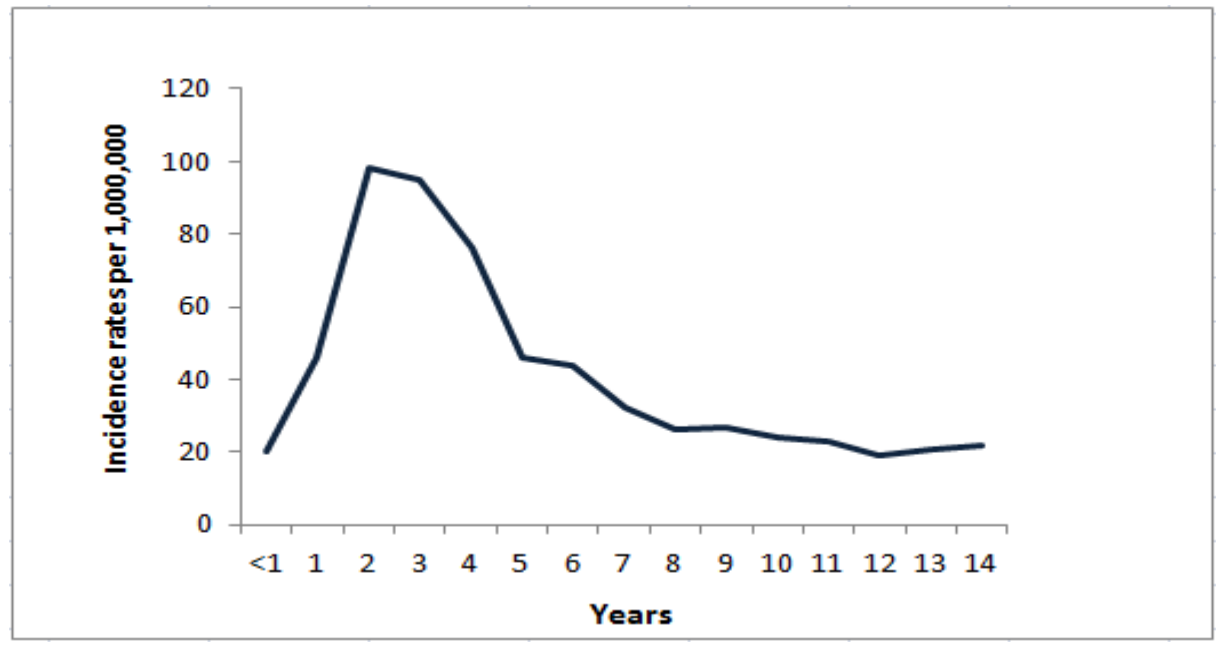

Figure 3. Age-specific incidence rates in childhood acute lymphoblastic leukemia by single year of age-at-diagnosis

Age-adjusted Surveillance, Epidemiology, and End Results cancer-incidence rates, 2006-2010, in childhood ALL, age 0-14 years. Incidence rates are per 1,000,000 per year and are age-adjusted to the United States standard population. Y-axis represents incidence rate per 1,000,000 per year and $\mathrm{X}$-axis represents age in years.

Source: National Cancer Institute, Surveillance, Epidemiology, and End Results ${ }^{5}$

\subsection{Ethnic differences in childhood acute lymphoblastic leukemia}

ALL risk is higher in Hispanics and Non-Hispanic White children than in any other ethnicity $^{30,32}$. African-Americans have the lowest risk although the mortality rate is higher in African American and Hispanic children ${ }^{3,33-35}$.

\subsection{Risk factors contributing to childhood acute lymphoblastic leukemia}

Although the pathogenic events behind the development of childhood ALL risk are still ambiguous, several congenital syndromes, such as Down syndrome, show significant associations with childhood ALL risk $^{1,36}$. The only known environmental risk factor is ionizing radiation ${ }^{1}$. Descriptive epidemiology consistently showed male gender $(\mathrm{M}: \mathrm{F}=1.2)$, age peak (1-5 years), White race (White: African American $=2.0)$ and to a 
Table 1. Risk factors (known, suggestive or with limited evidence) associated with childhood acute lymphoblastic

\begin{tabular}{|l|l|l|}
\hline Known & Suggestive & Limited evidence \\
\hline Gender (M:F $=1.2)$ & Maternal fetal loss & $\begin{array}{l}\text { Paternal smoking before } \\
\text { conception }\end{array}$ \\
\hline $\begin{array}{l}\text { Congenital disorders, ataxia-telangiectasia, } \\
\text { neurofibromatosis, Fanconi anemia and } \\
\text { Nijmegen breakage syndrome } \\
\text { pregnancy }\end{array}$ & $\begin{array}{l}\text { Mother older than 35 years at } \\
\text { Hydrocarbon } \\
\text { Paint } \\
\text { Motor vehicle exhaust }\end{array}$ \\
\hline White: African American $=2.0$ & & 60-Hz magnetic fields $>0.4$ uT \\
\hline Age peak ( 1-5 y) & & Postnatal chloramphenicol use \\
\hline Down syndrome & & Family history \\
\hline $\begin{array}{l}\text { Ionizing radiation } \\
\text { diagnostic, } \text { in } \text { utero }\end{array}$ & & $\begin{array}{l}\text { Decreased risk associated with } \\
\text { breastfeeding }\end{array}$ \\
\hline Birth weight $>$ 4000 g & & \\
\hline Higher socioeconomic status & & \\
\hline
\end{tabular}

$\mathrm{M}: \mathrm{F}$ indicates male-to-female ratio of incidence

Source: Linet MS et al. Interpreting epidemiological research: lessons from studies of childhood cancer. Pediatrics 2003; 112:218-232 22. 
degree, birth weight $\left(>4000 \mathrm{~g}\right.$ ) as risk factors for childhood ALL ${ }^{22}$. Several other epidemiological studies have found environmental exposure such as parental occupation $^{37,38}$, smoking, tobacco \& alcohol use ${ }^{38,39}$, maternal reproductive history ${ }^{40}$, maternal $\operatorname{diet}^{41}$, exposure to pesticides or solvents ${ }^{42,43}$, and exposure to residential powerfrequency magnetic fields ${ }^{44}$ to be associated with childhood ALL risk with inconsistent results $^{22}$. Most of these exposures are either maternal, during pregnancy, or early childhood. Studies suggest that the incidence of ALL is higher in developed countries than developing and under developed countries ${ }^{45}$. A list of risk factors that are known, suggestive or with limited evidence is provided in Table 1.

The types of cancers that most frequently develop in children are different from the most common adult cancers ${ }^{3,4}$. For example, although leukemia, lymphoma, and brain/other nervous system cancers account for more than half of all childhood cancers, they account for less than $10 \%$ of cancer cases in adults. In addition, etiologic differences and genomic variations within the same cancer type suggest that the childhood and adult cancers may be discrete diseases ${ }^{46}$. Because more than $95 \%$ of causal risk factors, including environmental risk factors, for childhood ALL are still unknown ${ }^{1}$, ${ }^{36}$, the current focus is on unraveling genetic risk markers ${ }^{36}$ to develop a predictive model and learn more about disease causation for preventive and therapeutic purposes.

\subsection{Statement of the problem and public health significance}

Among all childhood cancers, ALL alone accounts for more than $30 \%$ of cases in children up to 19 years of age, with approximately 3000 to 3500 cases diagnosed annually in the U.S. ${ }^{2-4}$. Over the past few decades there has been tremendous progress in 
early diagnosis and treatment of childhood cancers in the U.S. ${ }^{6,19}$ yet childhood cancers are still the leading cause of disease-related mortality ${ }^{3,4}$ Childhood leukemia is the most costly pediatric cancer to treat and average annual hospitalization cost is around $\$ 385.8$ million $^{47}$. The survival rate of childhood ALL has improved with new treatments, and current 5 year survival rate is around $90 \% \%^{5}$. According to Surveillance, Epidemiology, and End, Result (SEER) data (Figure 4), as of 2010, there were around 379,112 childhood and adolescent cancer survivors (diagnosed at age 0-19 years) and around 60,489 of them were ALL survivors ${ }^{2,3}$.

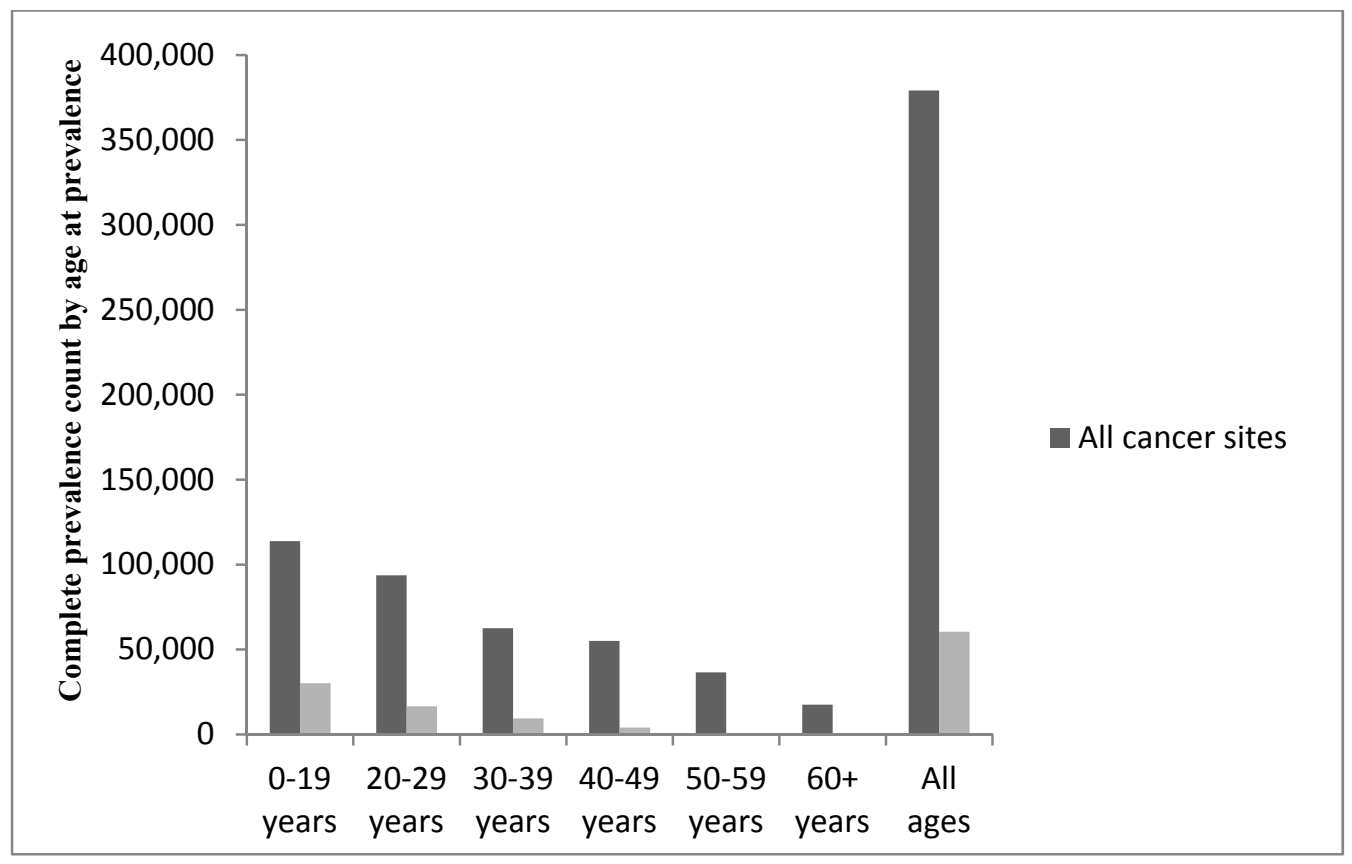

Figure 4. United States childhood cancer survivors

Number of people previously diagnosed with cancer as children in the United States and alive January 1, 2010. Y-axis represents complete prevalence count by age-at-prevalence and X-axis represents age group in years.

Source: National Cancer Institute, Surveillance, Epidemiology, and End Results ${ }^{5}$ 
As of January $1^{\text {st }} 2010$, more than $70 \%$ of childhood cancer survivors are 20 years of age and older and the majority of them are childhood ALL survivors. It has been estimated that, approximately 1 out of every 530 young adults between the ages of 20 years and 39 years is a childhood cancer survivor ${ }^{2,3}$. Childhood ALL survivors will soon be a significant proportion of young adults in the U.S., this suggests an immediate step towards prevention to reduce the increasing burden on public health is necessary.

Family burden, in the form of grief and financial burden starts at the time of diagnosis, when parents learn that their child has cancer and will go through necessary harm in order to survive ${ }^{7}$. It is important to recognize that "children are not little adults". Their bodies are still developing during illness and treatment. Therefore, cancer treatment affects them unequally over their life compared with adults. Although survival statistics have improved dramatically, the literature suggests that preventable suffering is a part of a childhood survivors' life ${ }^{8}$. It significantly affects the quality of life of these children and their families. The extreme cost of survival for some treatable cancers is one of gross mental impairment. Even after treatment, these children suffer from poor motor functioning and compromised social well-being for decades of life ${ }^{7}$.

For cancers, which show higher survival probabilities, such as childhood ALL, a significant proportion of survivors will have chronic and life altering side effects as a result of treatment ${ }^{7,8}$. Childhood cancer survivors continue to have social and behavioral difficulties ${ }^{9}$. Two-thirds of childhood ALL survivors also experience one or more permanent side effects of treatment such as heart dysfunction, neurocognitive impairment, osteonecrosis, osteoporosis, reproductive issues, severe growth retardation, permanent hearing loss, obesity or second malignant neoplasm ${ }^{6,8,10-16}$. 
Every year, more than 200,000 children under the age of 15 years are diagnosed with cancer all over the world ${ }^{8}$. Low-income countries represent four-fifths of all childhood cancers, and in the absence of best therapies and/or a very high cost, the mortality rate is significantly high $(>80 \%)$ in comparison with that of developed countries $(<20 \%)^{21,48}$. Therefore, an urgent action toward prevention is highly recommended by the International Society of Pediatric Oncology to reduce the mortality, life-long burden of survivors, burden on the health care system and society ${ }^{17}$.

A few genome-wide association studies (GWAS) have been completed in childhood ALL ${ }^{49-54}$. These studies yielded somewhat consistent associations, but with small effect sizes. Thus, the explained proportion of genetic susceptibility is very low. A recent re-analysis, however, showed that by considering all genotyped single nucleotide polymorphisms (SNPs) simultaneously, $24 \%$ of the total variation in precursor B-cell ALL risk is accounted by common genetic variation ${ }^{55}$. This result provides support for the involvement of many more SNPs than already identified in susceptibility to precursor B-cell ALL. The reasons for failure to obtain individual significant results are much debated ${ }^{56}$. One of the reasons that is most relevant to the current work is the possibility of associations masked by effect modification (interactions with other genes or environmental factors, gender or age) $)^{57}$.

\subsection{Consideration of study design}

We used a case-only design to see associations differing by gender and age groups. This study design is more efficient than other traditional epidemiological studies at detecting such effect modifications, which were the main focus of this study ${ }^{58}$. 
Recruitment of cases in a rare disease like childhood ALL is a challenge ${ }^{59}$, and hence, preferring a statistically powerful research design is more practical.

The case-only design is a transformation of the conventional case-control design to detect statistical interactions. An assumption of the case-only design, requires genotype and gender/age to be independent in the population i.e., genotype frequencies should not differ between genders or age groups in a healthy population. When this assumption is met, it is easy why a case-only study can be used instead of a case-control study $^{59}$.

Table 2. Odds ratio calculation for association of disease with gender, by genotype

\begin{tabular}{|l|c|c|c|c|}
\hline \multirow{2}{*}{ Gender } & \multicolumn{2}{|c|}{ Risk genotype positive } & \multicolumn{2}{c|}{ Risk genotype negative } \\
\cline { 2 - 5 } & Cases & Controls & Cases & Controls \\
\hline Male & $\mathrm{a}$ & $\mathrm{b}$ & $\mathrm{e}$ & $\mathrm{f}$ \\
\hline Female & $\mathrm{c}$ & $\mathrm{d}$ & $\mathrm{g}$ & $\mathrm{h}$ \\
\hline Odds ratio & \multicolumn{2}{|c|}{$\mathrm{ad} / \mathrm{bc}$} & \multicolumn{2}{|c|}{$\mathrm{eh} / \mathrm{fg}$} \\
\hline
\end{tabular}

Table 3. Odds ratio calculation for association of disease with gender and genotype, in cases and controls separately

\begin{tabular}{|l|c|c|c|c|}
\hline \multirow{2}{*}{ Gender } & \multicolumn{2}{|c|}{ Cases } & \multicolumn{2}{c|}{ Controls } \\
\cline { 2 - 5 } & $\begin{array}{c}\text { Risk } \\
\text { genotype } \\
\text { positive }\end{array}$ & $\begin{array}{c}\text { Risk } \\
\text { genotype } \\
\text { negative }\end{array}$ & $\begin{array}{c}\text { Risk } \\
\text { genotype } \\
\text { positive }\end{array}$ & $\begin{array}{c}\text { Risk } \\
\text { genotype } \\
\text { negative }\end{array}$ \\
\hline Male & $\mathrm{a}$ & $\mathrm{e}$ & $\mathrm{b}$ & $\mathrm{f}$ \\
\hline Female & $\mathrm{c}$ & $\mathrm{g}$ & $\mathrm{d}$ & $\mathrm{h}$ \\
\hline Odds ratio & \multicolumn{2}{|c|}{ ag/ce } & \multicolumn{2}{c|}{ bh/df } \\
\hline
\end{tabular}

In a traditional case-control study, interaction will be measured by their ratio adfg/bceh (Table 2), which is basically the ratio of two odds ratios (ORs) (ad/bc and 
$\mathrm{eh} / \mathrm{fg}$ ). Now, the same data is rearranged in Table 3. Again, interaction will be measured by the ratio agdf/cebh, which is the ratio of two ORs (ag/ce and bh/df) and equivalent to the OR derived in Table 2. According to the main assumption, genotype and gender/age should not be correlated ${ }^{60}$. In case of a rare disease, such as childhood ALL, disease-free controls would be representative of the normal population and second OR bh/df should be one, meaning genotype frequencies between two genders and age groups will be similar. This is the simplest rationale to explain the case-only design. It suggests that under the required assumption of test of independence, it is possible to estimate interactions from the cases-only design ${ }^{60}$.

In this scenario, we can estimate a statistical interaction from the association between genotypes and gender or age in the case-only study design, under the assumption that exposures and genotypes are independent ${ }^{60,61}$. There is no reason to believe that healthy males and female controls ${ }^{62}$, and healthy controls in two age groups (1-5 years and 6-10 years) have different genotype frequencies. The preference of the case-only design over the case-control is also due to its greater precision in estimating interaction (i.e., smaller standard errors) ${ }^{60}$ and smaller variance based on four cells of individuals (Table 4) instead of eight cells of individuals in the case-control design (Table 2) ${ }^{63}$.

Table 4. Odds ratio for association of disease with gender and genotype, in case-only studies

\begin{tabular}{|l|c|c|}
\hline \multirow{2}{*}{ Gender } & \multicolumn{2}{|c|}{ Cases } \\
\cline { 2 - 3 } & $\begin{array}{c}\text { Risk genotype } \\
\text { positive }\end{array}$ & $\begin{array}{c}\text { Risk genotype } \\
\text { negative }\end{array}$ \\
\hline Male & $\mathrm{a}$ & $\mathrm{e}$ \\
\hline Female & $\mathrm{c}$ & $\mathrm{g}$ \\
\hline Odds ratio & \multicolumn{2}{|c|}{$\mathrm{ag} / \mathrm{ce}$} \\
\hline
\end{tabular}


This study design is only good to calculate OR for interactions ${ }^{61,64}$. It cannot calculate OR for the main effects of genotype (ORg) or gender/age (ORe) individually. Our main interest is in gender- and age-specific risk markers, and we were not interested in the main effects.

The statistical power of the case-control design and the case-only design was compared in a study by hypothetical (200 cases and 400 controls vs 200 cases) and empirical data (four case-control studies) ${ }^{65}$. Although results showed similar ORs for interaction in both study designs, the case-only design had a much narrower $95 \%$ confidence interval. Similarly, the four case-control studies also showed no substantial difference in OR for interaction, but confidence intervals were narrower in the case-only analysis. It suggests that the case-only study design has greater statistical power for detecting gender-genotype or age-genotype interactions with smaller sample size in comparison to a case-control study design ${ }^{65}$.

Table 5. Odds ratio for association of disease with age and genotype, in case-only studies

\begin{tabular}{|l|c|c|}
\hline \multirow{2}{*}{ Age } & \multicolumn{2}{|c|}{ Cases } \\
\cline { 2 - 3 } & $\begin{array}{c}\text { Risk genotype } \\
\text { positive }\end{array}$ & $\begin{array}{c}\text { Risk genotype } \\
\text { negative }\end{array}$ \\
\hline Age (1 to 5 years) & $\mathrm{a}$ & $\mathrm{e}$ \\
\hline Age (6 to 10 years) & $\mathrm{c}$ & $\mathrm{g}$ \\
\hline Odds ratio & \multicolumn{2}{|c|}{ ag/ce } \\
\hline
\end{tabular}

A case-only study was also used to examine the age-specific associations with childhood ALL. In this scenario, we used the same approach as it has been explained earlier for gender (Table 5). Again, in the case of effect modification by age, the case- 
only approach provides more statistical power than traditional case-control design when the case group is split into two age groups.

\subsection{Structure of the dissertation}

This dissertation is written in a traditional style which contains eight chapters including this chapter. Following this chapter, Chapter 2 comprehensively reviews literature relating to gender- and age-specific associations in general, including childhood ALL. The purpose of the literature review is to provide insight that genetic architecture can significantly contribute to gender- and age-specific risk associations in childhood ALL. Chapter 3 explains hypothesis and specific aims of the study.

Chapter 4 provides an overview of the methods used for the gender- and agespecific associations in childhood ALL. It mainly addresses: rationale for quality controls (QCs) to assess the sample, genotyping method, and generated results, statistical analyses used to identify gender- and age-specific associations. Bioinformatic and empirical analyses were also performed for all identified markers to assess their functionality as explained in Chapter 4.

All gender- and age-specific association results and bioinformatic analyses of identified markers are provided in Chapter 5. Relevant tables with interaction ORs and interaction $\mathrm{P}$ values are provided at the end of Chapter 5. Chapter 6 provides a detailed discussion of identified genetic markers or genes and their possible relevance to genderor age-specific associations. Limitations and strengths of the present study are provided in Chapter 7. Conclusion is explained in Chapter 8. 
All tables and figures are provided in the relevant section of the chapters or in the appendix. All references are provided at the end of the dissertation.

\subsection{Significance of the dissertation}

This dissertation mainly focused on gender- and age-specific risk markers. This was the first GWAS of childhood ALL specifically addressing these issues. Gender difference is an underappreciated and understudied research area in cancer research ${ }^{26}$. Findings from this dissertation may inform future studies of childhood ALL as well as other diseases, which show significant gender and age differences.

In majority of developing and underdeveloped countries, childhood cancers are not considered a major public health issue because of the high numbers of deaths from infectious diseases ${ }^{66}$. Most importantly these countries also represent a higher percentage of younger population. Recent studies suggest that childhood cancers are among top five leading cause of death in children in developing and underdeveloped countries ${ }^{2}$. Even

countries like U.S. invest little on childhood cancer prevention ${ }^{67}$. Childhood cancers, gender and age difference in cancers are major public health issues and medical concern. Therefore, this research and the results from this project addressed a major public health issue aiming to elucidate currently unknown risk factors for childhood ALL.

\subsubsection{Better understanding of gender- and age-disparities in childhood acute lymphoblastic leukemia}

Although descriptive epidemiological data have shown a significant role of gender and age specificity in childhood ALL, to date there have been few examples of gender- and age-specific associations. Our main interest was, therefore, the detection of 
association differing in directions between males and females (risk vs protection), means risk association for one gender and protective association for another. Interaction with gender implies statistically significant differences in male- and female-specific ORs which is basically a difference in magnitude (for example, an $\mathrm{OR}=1.5 \mathrm{in}$ females and 3.5 in males and $\left.\mathrm{P}_{\text {interaction }}<0.05\right)$. This type of quantitative interaction in the overall analysis would yield an OR that is approximately the average of the two gender-specific ORs. We were interested in qualitative interactions that would be missed in the overall analysis (like in previous GWASs) ${ }^{50-53,68}$. A qualitative interaction is a result of two ORs differs in their directions (risk vs protection) rather than being in the same direction (differ in magnitude). Cross-over effect (association differing in direction) usually results in an $\mathrm{OR}$ close to the unity $(\mathrm{OR}=1.0)$ with no statistical significance in the overall analysis. Such SNPs are typically dismissed for any further consideration. Genderspecific associations, differing in direction, are difficult to identify, even in secondary analysis, unless all of the raw data are re-analyzed. Associations differing in direction are simple examples of an effect modification where a polymorphism shows a risk association in one gender and a protective association in the other. Our main objective was to unravel associations differing in direction that would have remained undetected in the overall analysis.

A list of previously identified childhood ALL risk markers in GWAS are reported in the appendix Table 1. Our long-term aspirations are to learn more about the gender and age disparity and exploit this information to implement preventive and therapeutic measures. Because males are more prone to develop ALL and more likely to have unfavorable clinical outcome, it may appear that only males will benefit from the results. 
This is not our thinking, and we believe that any information on disease pathogenesis will have multiple beneficiaries including other people with other childhood and adult cancers that show differential distribution between genders and among age groups.

\subsubsection{Guidance for new strategies for studying other complex diseases}

The case-only design circumvents several limitations of the traditional casecontrol design in interaction analysis. Successful application of this approach may be conducive, not only for childhood ALL, but also for research on other rare, gender-and age-specific disorders. It is well known that the time course and cost of recruiting subjects is hard for a prospective cohort study in case of rare diseases. Therefore, this project could provide guidance for new strategies to investigate an unidentified component of genetic susceptibility and effect modification in more common complex diseases. 


\section{REVIEW OF LITERATURE}

Descriptive epidemiological data have consistently provided evidence for gender and age differences in distribution of nearly all cancers ${ }^{18,24,69}$. Males generally show higher susceptibility, mortality and worst survival in most cancers. Studies that exploited gender differential for etiological clues identified several gender-specific risk markers ${ }^{68 \text {, }}$ ${ }^{70-74}$. Similarly, studies that used age differential for clues also unmasked risk markers for early or late-onset of diseases ${ }^{75-78}$. Prior to the present study, there was no GWAS in childhood ALL that has used a gender- and age-specific approach to identify markers for childhood ALL susceptibility. Furthermore, a time-to-event analysis approach was used to unravel risk markers that might be associated with early-onset of childhood ALL risk.

\subsection{Genome-wide association studies}

The National Institute of Health (NIH) defines GWAS as a study of common genetic variations across the entire genome designed to identify genetic associations with observable traits $^{79,80}$. A GWAS uses high-throughput genotyping technology. It examines thousands of polymorphisms to relate them to a clinical condition or measurable traits ${ }^{80}$. It is a hypothesis-free approach to scan markers across the whole genome. There are millions of genetic variants in the human genome. A traditional case-control study design is the most common approach in GWASs. GWASs also use stringent QC protocols to reduce the error in the study ${ }^{81}$. These QC protocols assess the sample, genotyping methods and generate results for potential causes of systematic error ${ }^{82}$. All QCs measured in this case-only GWAS are explained in Chapter 4. 
The human genome contains around 19,881 genes ${ }^{83,84}$. These genes are discrete segments of deoxyribonucleic acid (DNA) that convey information to protein via ribonucleic acid (RNA). Genetic variation refers to DNA sequence polymorphism ${ }^{83}$. An allele is one of the several forms of a DNA segment (e.g. a gene or locus).

There are many forms and levels of genetic variations ${ }^{83}$. The most common genetic variation that has been examined in this study is called a SNP. For example, the same nucleotide position may be occupied by a guanine $(\mathrm{G})$ in one individual and a cytosine (C) in another. An allele, which occurs less frequent in a population is called a minor allele. In the example given below, $\mathrm{C}$ is the minor allele, and polymorphism will be represented as $\mathrm{G}>\mathrm{C}$ polymorphism.

\section{Subject 1: ACTGACTCAT \\ Subject 2: $\quad$ ACTCACTCAT}

In this instance, the two nucleotides represent two different alleles. These SNPs occur in every 100 to 300 nucleotides on average in human genome. It has been estimated that number of SNPs in the human genome is up to 40 million. The most common variation of a SNP has two alleles. There are three possible genotypes from a SNP with two alleles. Genotype is the genetic constitution of an organism specifying the particular alleles at a defined locus in the genome. It is basically two alleles inherited at a specific locus. Human genome contains 22 pairs of autosomal chromosomes and a pair of sex chromosomes. Males have one $\mathrm{X}$ and one $\mathrm{Y}$ chromosome, and females have one pair of $\mathrm{X}$ chromosomes. So, the genotype is the combination of two alleles, one from each chromosome. For example, the three genotypes in a population are: 
Subject $1=\mathrm{GG}$ (wild-type), both chromosomes have $\mathrm{G}$ allele and $\mathrm{G}$ allele is commonly present in the population

\section{ACTGACTCAT \\ ACTGACTCAT}

Subject $2=$ GC (heterozygote), one chromosome has G allele and another chromosome has $\mathrm{C}$ allele.

\section{ACTGACTCAT \\ ACTCACTCAT}

Subject $3=\mathrm{CC}$ (homozygous variant), both chromosomes have $\mathrm{C}$ alleles, and $\mathrm{C}$ allele is less frequently present in the population.

\section{ACTCACTCAT \\ ACTCACTCAT}

The wild-type genotype is the referent group in a statistical analysis. The other two genotypes may be included in the analysis as they are (gene dosage analysis) or may be pooled depending on the genetic model. Genetic models are explained in Chapter 4. Most genetic associations are mediated by gene expression changes due to genetic variation and their effect may be opposite in males and female ${ }^{83,85,86}$.

\subsection{Gender disparity in cancer incidence}

Gender is an important risk factor in disease pathogenesis ${ }^{24,32,68,87,88}$. Studies clearly suggest biological, physiological and behavioral differences between males and females, including differences in the incidence, progression, mortality and survival of many diseases ${ }^{24,26,32,86}$. Males have a higher life time probability of developing cancer than females $(43.9 \% \text { vs } 38.0 \%)^{4}$. The National Cancer Institute (NCI) SEER database shows that males consistently have a higher incidence (males: 542.3 vs females 418.8 per 
$100,000)$ and mortality rates (males: 215.3 vs females: 149.7 per 100,000$)^{4,24}$. Incidence rates were age-standardized to the 2000 US standard population and are for all cancers for 2006-2010. Males also show worse survival than females. Cook et $\mathrm{al}^{24}$ in a recent study used SEER data to calculate gender-specific incidence rates and male-to-female incidence rate-ratios (IRR) for specific cancer sites for the period 1975 to 2004. Only five cancers (breast, gall bladder, thyroid, pertitoneum/omentum/mesentry, and anus/anal canal/anorectum) were significantly higher in females when compared with males ${ }^{4}$. The leading cancers also show a higher male-to-female (M:F) ratio: lung and bronchus: 1.45; colorectal cancers: 1.32 ; urinary bladder: 4.05 ; non-Hodgkin lymphoma (NHL): $1.43{ }^{4}$. Males consistently show higher cancer risk in 32 of 35 cancer sites, excluding sexspecific cancers, across geographical regions ${ }^{25}$.

Likewise, for all childhood cancers, males show a higher incidence rate than females $(178.0 \text { vs } 160.1 \text { per } 1,000,000)^{3}$. Incidence rates were age-standardized to the 2000 US standard population and are for all cancers for 2006-2010. These observations suggest the importance of analyzing results by gender, and how it may provide a fundamental understanding of human physiology, pathology and pharmacology that will ultimately benefit both genders ${ }^{88}$. Cancer epidemiology has been more concerned with disease etiology than disease heterogeneity and failed to examine gender as a major risk factor $^{24}$. Adjusting the results for gender does not explain gender difference in susceptibility of a disease, it rules out confounding by gender ${ }^{26}$. Stratified analysis by gender is one approach to unravel a gender-specific association, which can be confirmed by a statistical interaction analysis. Thus, gender-specific approach may provide important etiological clues in disease pathogenesis, progression, and treatment. Examples 
include liver, colon and thyroid cancer. Higher interleukin-6 (IL6) levels may be one reason for higher incidence of hepatocellular carcinoma (HCC) in males ${ }^{89,90}$. A study on endogenous hormones suggests the protective role of estrogen in women through suppressing IL6 mediated inflammation of the liver. On the contrary, testosterone can increase liver cell proliferation in men. Likewise, males again show higher incidence rate than females to develop colon cancer ${ }^{4}$. Estrogen plays a protective role in women for the development of colon cancer, and estrogen replacement therapy further reduces the incidence of colorectal cancer in postmenopausal women ${ }^{91,92}$. The androgen receptor expression in thyroid follicular cells may be responsible for higher incidence of thyroid follicular cancer in females ${ }^{93,94}$. These physiological differences elucidate the importance of gender difference in cancer. Thus, it would be difficult to predict a disease risk with the same accuracy in both genders, if results are not analyzed by gender ${ }^{68}$.

\subsection{Gender disparity in other diseases}

Gender differences are also common in other diseases. Like cancers, susceptibility to infections is greater in males from birth ${ }^{95,96}$. For example, incidence of septicemia, meningitis and tuberculosis is higher in males from infancy to childhood. Moreover, males have a poorer immune response than females to several bacterial and viral infections ${ }^{87,97-101}$. Gender differences in infections are similar to cancer. Immune surveillance mechanisms are similar against cancers and infections. This suggests that the difference in immune system activity between males and females may play a significant role in gender differences. As in cancers, only few infections show higher morbidity and

mortality rates in females, such as pertussis and mycoplasma pneumonia ${ }^{102,103}$. Nearly all 
human diseases show gender-specific differences in prevalence, age of onset and/or severity. In utero loss is also more prominent with the male fetus ${ }^{104}$. In comparison to males, average life span of females is longer ${ }^{105}$, and not only they are healthier, but the survival rates from infectious diseases, sepsis, trauma or injury are also higher ${ }^{106,107}$.

The immunological disadvantage of being males in comparison to females is well known $^{96,108}$. Lower production of antibodies has been noted in males in comparison to females. In general, females have a better innate and adaptive immune system ${ }^{109}$. These also contribute to higher incidence of autoimmune and inflammatory diseases in females. For example, autoimmune thyroid diseases (Hashimoto thyroiditis and Graves' disease), systemic lupus erythrmatosus, Sjogren syndrome, multiple sclerosis, primary biliary cirrohsis and celiac disease are more common in females ${ }^{110}$. The X chromosome contains the largest number of immune-related genes in the whole human genome ${ }^{96}$. The difference in $\mathrm{X}$ chromosome numbers results in immunological differences in males and females despite the presence of dosage compensation mechanisms in females ${ }^{96,111}$. X-linked primary immunodeficiencies are typical examples of gender difference and the disadvantage of being male ${ }^{96}$. Mutations or polymorphisms of an X chromosome-linked gene phenotypically expressed in males, who then show functional impairment of the respective proteins leading to diseases such as primary immunodeficiencies ${ }^{96,111}$.

Environmental and occupational exposures are commonly believed to play a significant role in gender differences in diseases. The male excess in cancers may be due to some environmental factors, including smoking, diet and life style, but environmental variations alone cannot explain gender difference in cancer risk ${ }^{26}$. Thus, genetic factors 
may shed some light on male excess in cancers. The examples given above suggest that physiological differences, immune system, $\mathrm{X}$ chromosome number, environmental risk factors and genetics may affect the disease susceptibility with gender specificity. Most of the endogenous causes of gender differences in disease are still unknown. In comparison to most of these endogenous causes, such as sex hormones and immune functions, the genetic contribution to gender difference, until recently, is an understudied research area. A stratified result by gender would produce more meaningful results, if one gender has a higher risk.

To understand the biology of sex and gender difference, the Institute of Medicine (IOM) formed a committee in November $1999^{112}$. The committee issued three common messages:

1- Sex matters.

2- The study of sex difference is evolving into a mature science.

3- Barriers to the advancement of knowledge about sex differences in health and illness exist and must be eliminated.

It is important to understand that gender differences in health and disease need scientific inquiry in all aspects of biomedical, health-related and epidemiological research. The committee made several recommendations including ${ }^{112}$ :

1- Promote research on sex at the cellular level. Determine how genetic sex differences influence other level of biological information, including susceptibility to disease, and develop systems that can identify and distinguish between the effects of genes and the effects of hormones. 
2- Study sex differences from womb to tomb. Inclusion of sex as a variable in basic research design.

3- Investigate natural variations. Examine genetic variability, disorders of sex differentiation, reproductive status, and environmental influences to better understand human health.

4- Monitor sex differences and similarities for all human diseases that affect both sexes.

Therefore, further studies designed to look for possible gender differences in all clinical and epidemiological research has been encouraged.

\subsection{Genetic basis of gender disparity}

The role of sex hormones, oxidative stress, chromosomal telomere length, iron level, and immune function are thought to be the reasons for gender differential in human diseases $^{113}$. Genetic contribution to gender differences, however, has rarely been explored. Although the role of X chromosome is mainly hypothesized for gender-specific gene expressions, autosomal chromosomes show gender-specific gene expression patterns $^{86,96,114}$. It has been assumed that autosomal genome is similar between males and females. However, studies suggest that genetic variations within autosomal genomes affect anatomical, behavioral and physiological traits differently in males and females ${ }^{86}$, 114-117. Thus, potential of a polymorphism to show a gender-specific association does not depend on its genomic location ${ }^{26}$.

Recent evidence suggests that the regulatory genome is sexually dimorphic ${ }^{118}$ i.e., there is a phenotypic difference between the two genders. Most phenotypic sexual 
dimorphisms, including gender-specific effects on diseases, are a result of gender-specific differences in gene expression regulation. Sexually dimorphic gene expression at messenger RNA level has been observed in a wide range of organisms, including primates, rodents, fish, worms and flies ${ }^{114,118-121}$. A mouse study on gender-specific gene expression identified thousands of gender-biased genes in autosomes ${ }^{119}$. Thus, mammalian genomes are enriched with gender-biased genes that show gender-specific expression. These animal models also suggest that genotype gender-interactions are prevalent and that genetic contribution to disease etiology will be masked if genderspecific analysis is overlooked.

There are, however, very few examples of documented genetic basis behind a gender differential in humans, presumably due to lack of analysis by gender. Studies that have used gender-specific approaches have so far successfully unmasked a number of gender-specific risk markers in childhood ALL (Table 6) ${ }^{122-131}$ and in other diseases ${ }^{132-}$ ${ }^{138}$. For example, two ARID5B (AT rich interactive domain 5B (MRF-1 like)) polymorphisms (rs10994982 and rs 10740055), previously identified as a risk marker in childhood ALL in GWAS ${ }^{51,52}$ (Table 6), yielded significant gender-specific associations $\left(\mathrm{P}_{\text {interaction }}=0.01 \text { and } \mathrm{P}_{\text {interaction }}=0.03 \text {, respectively }\right)^{139}$ (Table 7). These SNPs were significantly associated with risk only in males $(\mathrm{OR}=3.79$ and $\mathrm{OR}=4.35$, respectively; ORs for females were: 1.03 and 1.37). Other ARID5B polymorphisms did not show any statistically significant interactions, but males had a higher risk (Table 7). However, one major limitation of this study is selection of controls, as controls are mainly adults (median age 30.1 years vs 4.2 years in cases) and recruited at locations different from childhood ALL cases. Thus, gender-specific results might be affected by selection bias, 
Table 6. SNPs associated with gender-specificity in childhood acute lymphoblastic leukemia risk

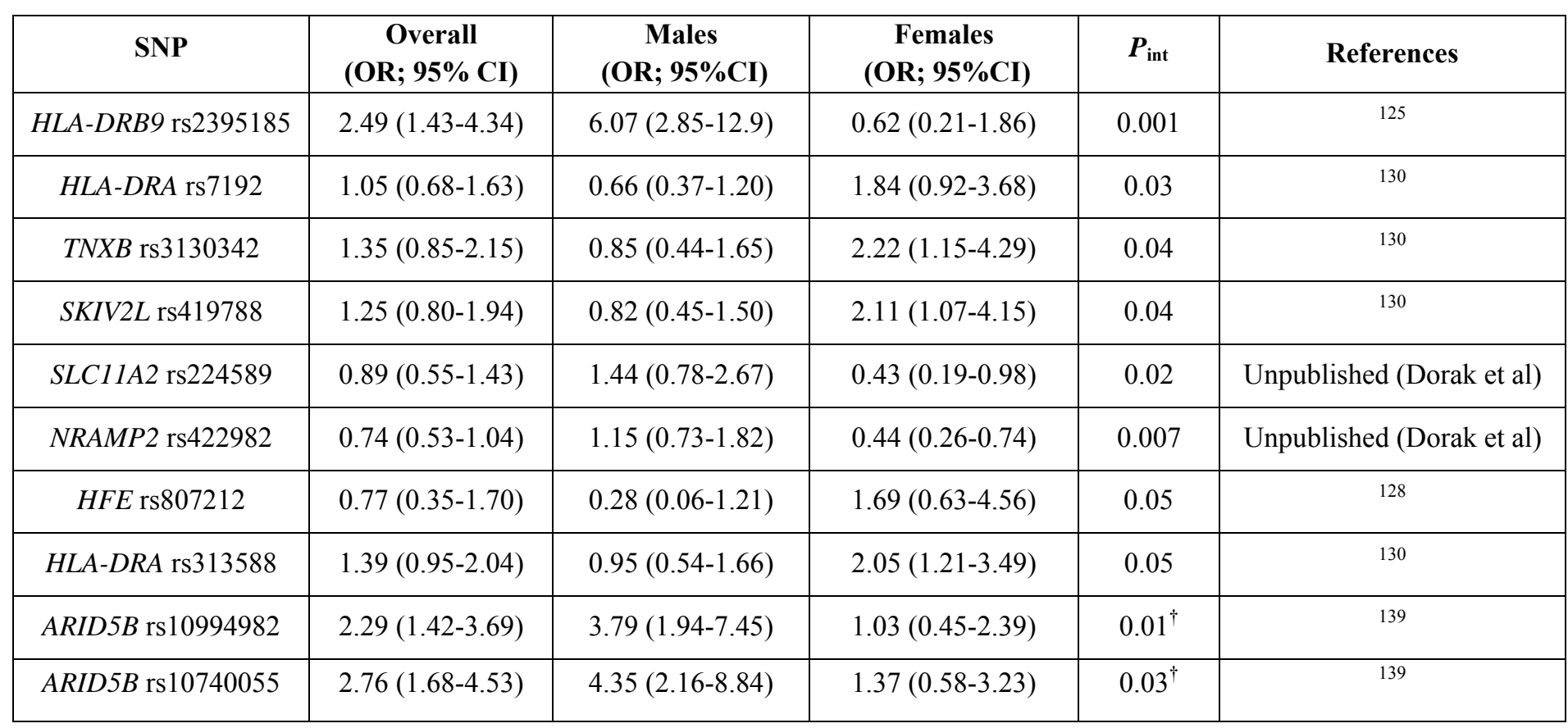

$\mathrm{SNP}=$ single nucleotide polymorphism; $\mathrm{OR}=$ odds ratio; $\mathrm{CI}=$ confidence interval; $P_{\mathrm{int}}=\mathrm{P}$ value for interaction

${ }^{\dagger} \mathrm{P}$ values denotes the Mantel-Haenszel $\chi^{2}$ test of homogeneity for risk differences between males and females 
Table 7. SNPs associated with childhood acute lymphoblastic leukemia risk in previous genome-wide association studies with gender- and age-specificity

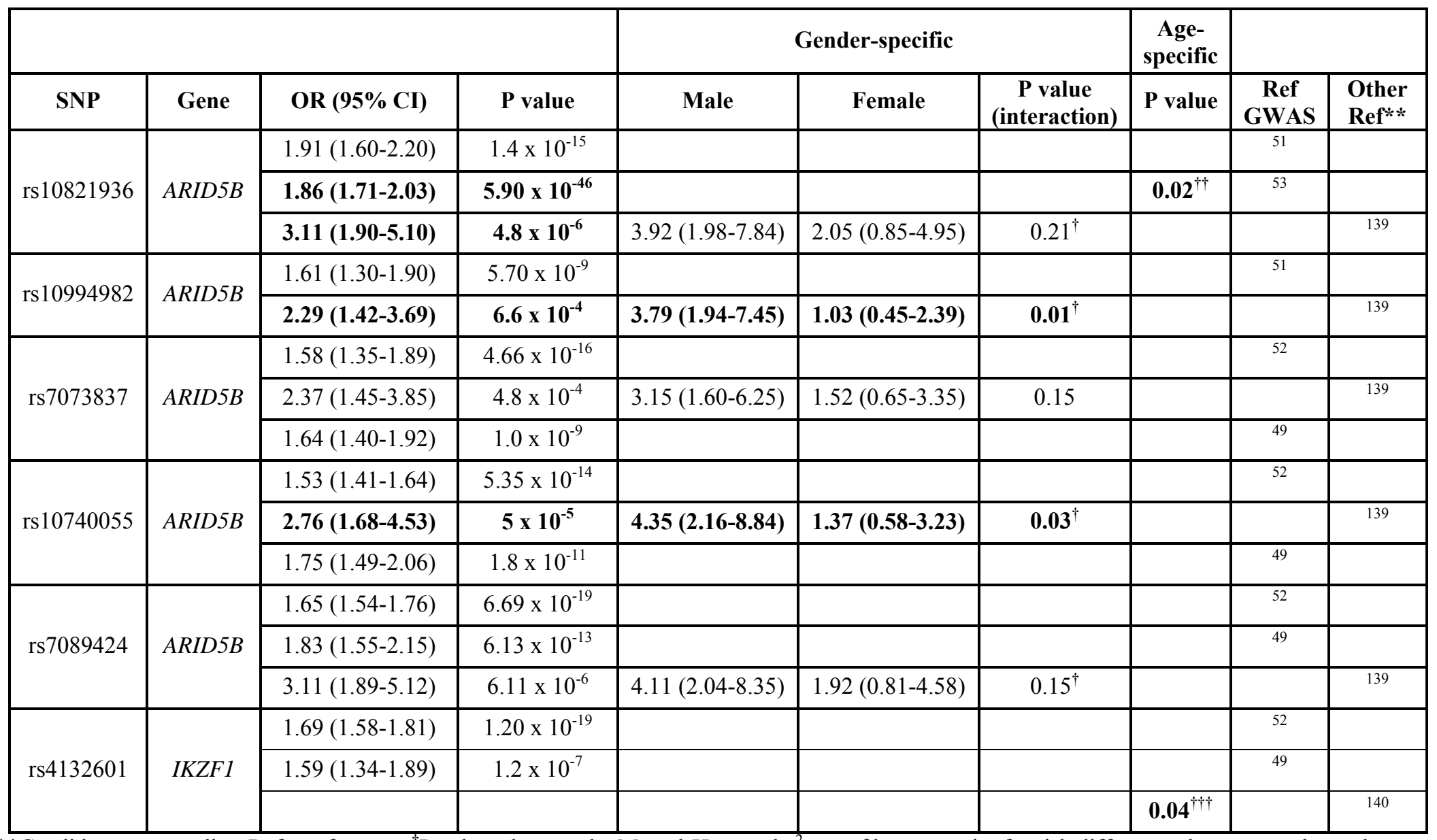

**Candidate gene studies, Ref = reference; ${ }^{\dagger} \mathrm{P}$ values denotes the Mantel-Haenszel $\chi^{2}$ test of homogeneity for risk differences between males and females; ${ }^{\dagger}$ Comparison between age group before $10 \mathrm{y}$ and older than $10 \mathrm{y} ;{ }^{\dagger \dagger}$ Recessive model (TT+GT vs GG) yielded significant association (3.83 year vs 5.58 year) 
as these controls may have different environmental exposures than childhood ALL cases. Re-analysis of GWAS datasets revealed 12 polymorphisms in: coronary artery diseases (CAD; rs7865618), Crohn disease (CD; rs3792106 and 6431654), rheumatoid arthritis (RA; rs3134926, rs4947244 and rs3132666) and type1 diabetes (T1D; rs2763979, rs4148873, rs3131622, rs480092, rs3134926 and rs12660883) that show gender-specific associations with increased disease risk in only one gender ${ }^{68}$. A study on rs3792106 and CD successfully replicated gender-specific association (OR heterogeneity $\mathrm{P}$ value 0.04 ; OR for females $=1.48, \mathrm{P}=6.9 \times 10^{-13} ; \mathrm{OR}$ for male $\left.=1.22, \mathrm{P}=0.01\right)^{141}$. These examples emphasize the importance of gender-specific analysis.

Gender differences play a critical role in drug efficacy and safety profile, and are well recognized in drug pharmacokinetics and pharmacodynamics ${ }^{142}$. Women are at far greater risk than men of developing unfavorable outcome to some drugs ${ }^{88,142-144}$. Many drugs for cardiovascular disorders show significant gender differences in their pharmacokinetics profiling $^{142}$. For example, CYP (cytochrome P450) genes show significant gender-specific activity differences. CYP1A2, CYP2E1 and CYP2D6 genes, all have higher activity in males, but CYP3A4 has higher activity in females. These results suggest that gender plays a significant role on CYP genes expression levels ${ }^{142}$.

Another example of a gender-specific association is glucocorticoid treatment. Expression of specific genes is altered in response to glucocorticoid treatment, but in opposite directions in males and females ${ }^{145}$. It has been suggested that anti-inflammatory actions of glucocorticoid are more effective in males. These studies suggest that physiological phenomenon, such as sex hormones and immune system, that differs 
between males and females provide the basis for gender-specific genetic associations with disease susceptibility ${ }^{86,88}$.

The translation of gender-specific gene expression differences at the protein level is imperative ${ }^{26}$. Studies that compared the proteome of males and females like transcriptomic studies, also identified prominent gender differences ${ }^{117}$. Likewise, a study unmasked remarkable gender differences in serum metabolite concentrations. A total of 102 out of 131 metabolites were statistically different between males and females $(\mathrm{P}<$ $3.8 \times 10^{-4}$; Bonferroni correction threshold) and were correlated with genetic variants in metabolism-related genes ${ }^{146}$. It suggests sexual dimorphism is also present at the metabolomic and proteomic level. To date, no systematic survey has addressed genderspecific associations in cancer ${ }^{26}$. Thus, gender-specific analysis may help to identify some of the missing heritability in GWAS ${ }^{56,68}$. Sexual dimorphism in gene expression may result in such associations (Figure 5), which has been recognized as a common occurrence $^{26,86,147,148}$.

It has been suggested that "one potential source of genetic variation that may contribute to missing heritability is the associations which differ in magnitude and/or direction between males and females" ${ }^{57}$. 


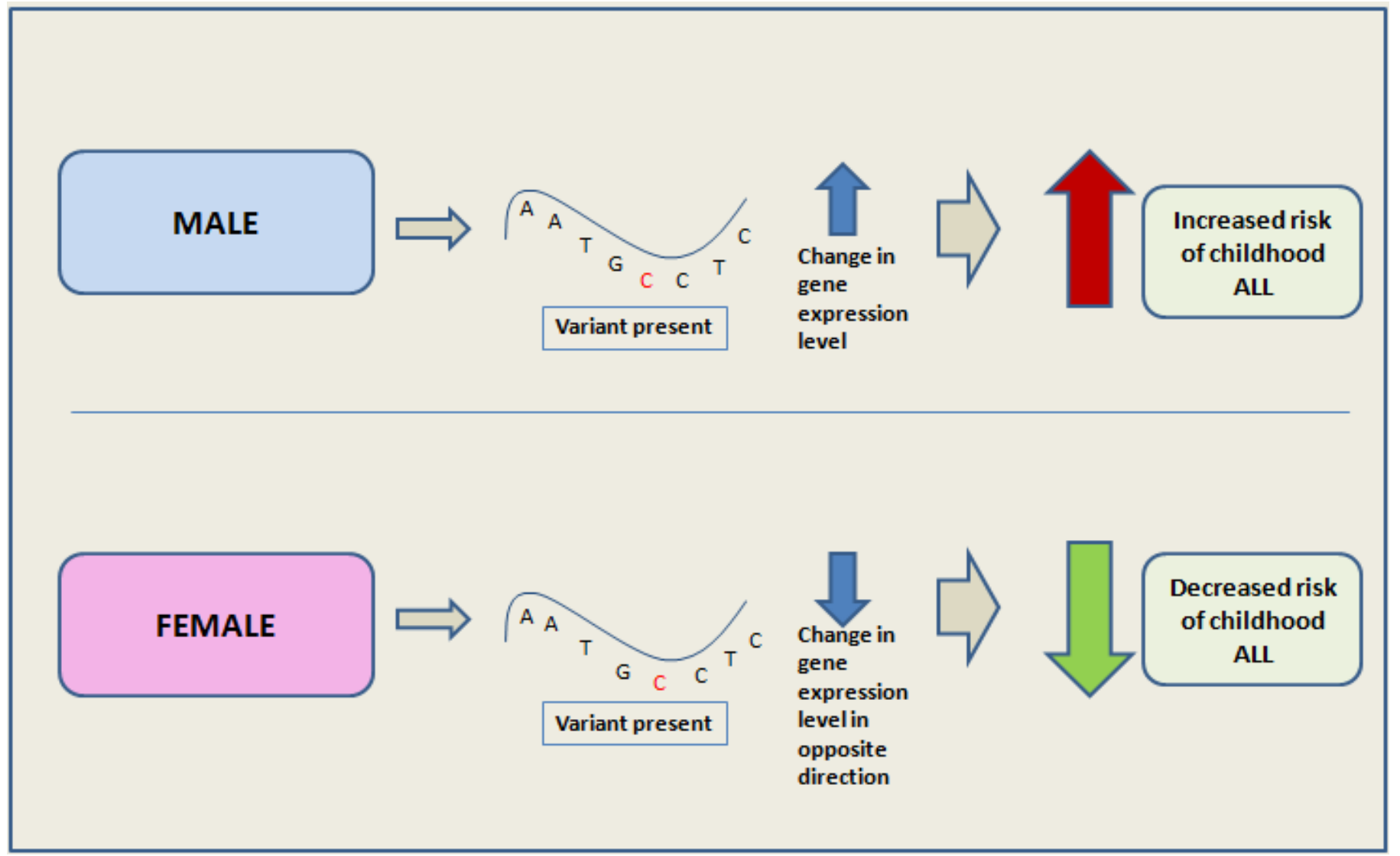

Figure 5. Genetic variants may be either protective or risk markers for childhood ALL depending on the presence or absence of environmental risk factors

Note: Most genetic associations are mediated by gene expression changes due to genetic variation and their effect may be different in males and females. 


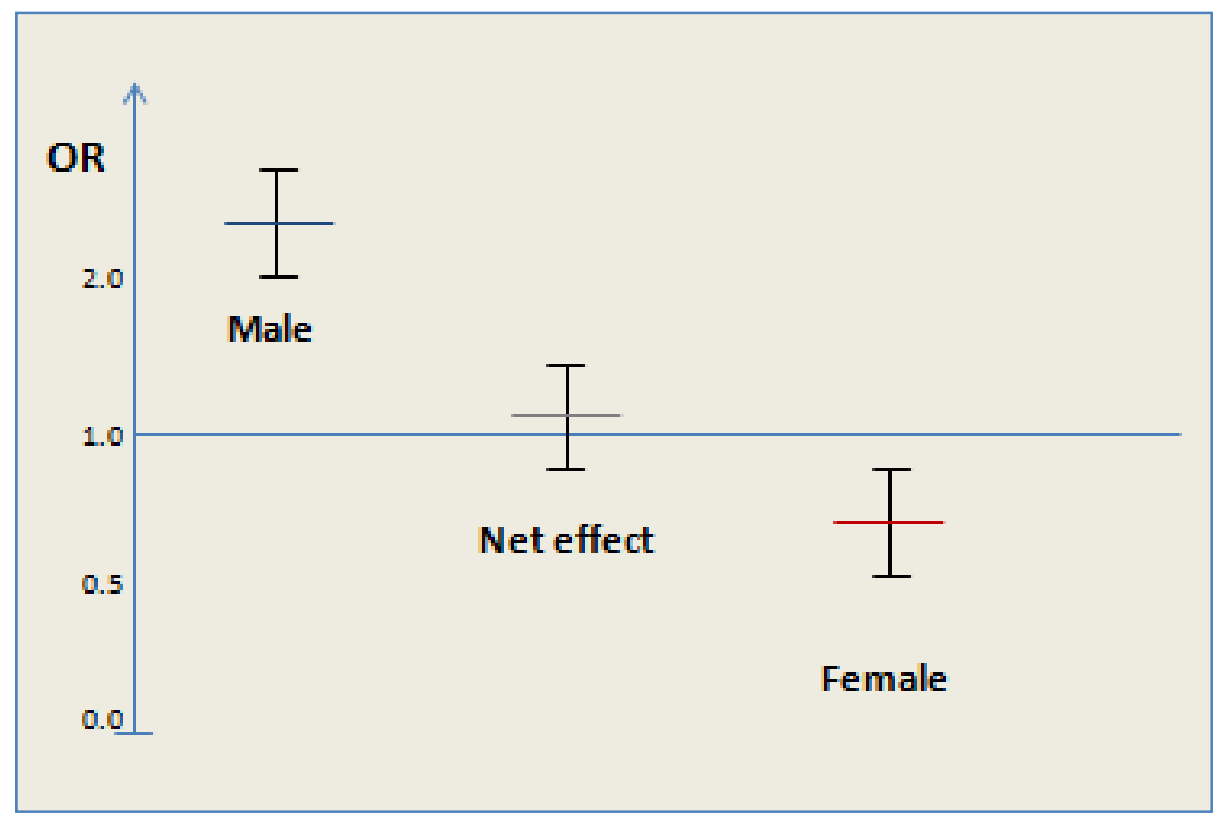

Figure 6. Difference in the direction of a gender-specific association for childhood ALL Note: Males show a risk association and females show a protective association after stratified analysis by gender. Net effect (odds ratio) is close to one and statistically nonsignificant. $\mathrm{OR}=$ odds ratio

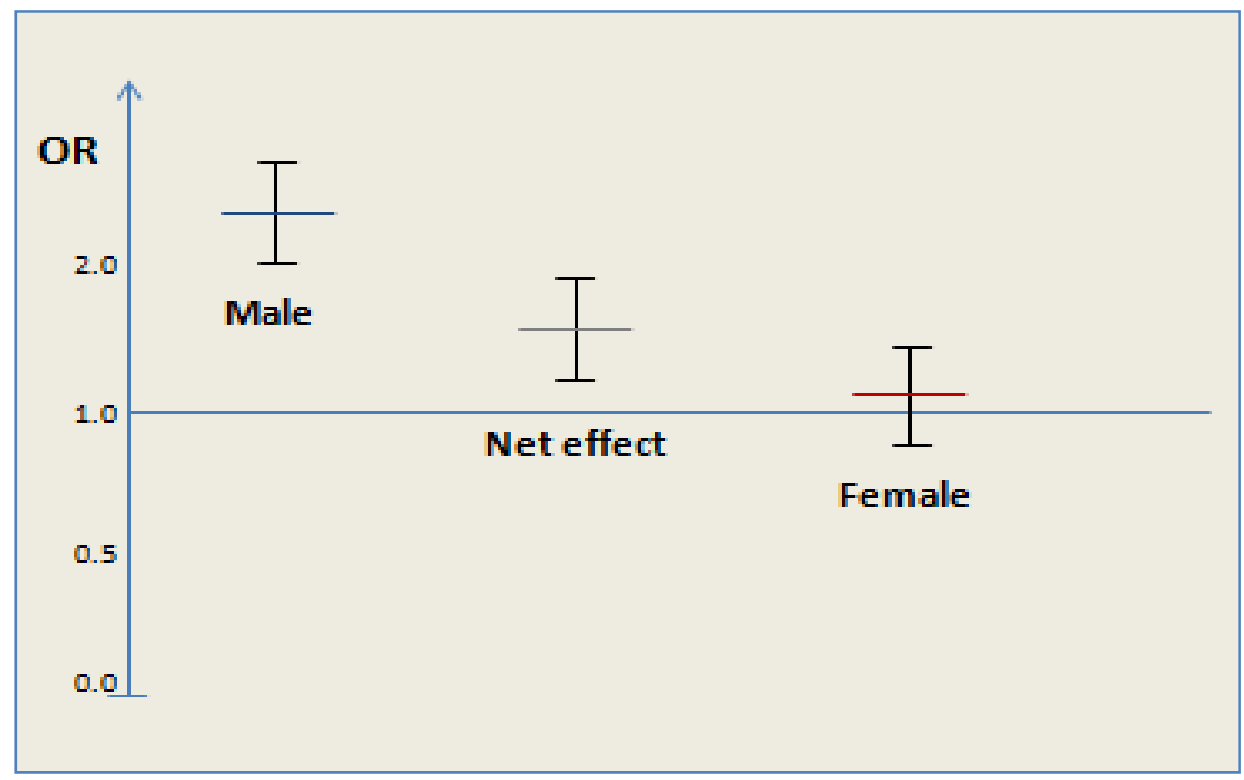

Figure 7. Difference in the magnitude of a gender-specific association for childhood ALL Note: Males show a risk association and females do not show any association after stratified analysis by gender. Net effect (odds ratio) shows an average of two odds ratio. OR $=$ odds ratio 
In gene expression or genetic association studies, two kinds of gender differences are possible ${ }^{26}$ : associations differing in direction (Figure 6) or differing in magnitude (Figure 7). The main interest of the present study was to identify the associations that differ in direction between males and females. This type of association is called "sexually antagonistic interaction", "cross-over effect" or "qualitative interaction". The examples stated below may arise from known differences in human physiologic functions and gene expression levels between males and females ${ }^{26}$.

Several examples of associations differing in direction between males and females in genetic associations have been reported ${ }^{86}$. The most remarkable one differing in direction concerns the genetic regulation of genetic recombination rates in the human genome $^{149}$. A GWAS designed to examine the genetic basis of recombination rate differences has shown an association differing in direction between males and females in RNF212 (ring finger protein 212) SNPs ${ }^{149}$. Two RNF212 SNPs (rs3796619 and rs1670533) are associated with higher recombination rate in males. The same two SNPs were associated with low recombination rate in females. These associations were replicated successfully in an independent sample in the same study ${ }^{149}$. It was suggested that if the frequency of the risk markers changes, the average recombination rate will increase for one gender and decrease for the other, but the gender-averaged recombination rate of the population can stay relatively constant. The presence of similar robust and replicated associations in IFNG (interferon gamma) polymorphism (rs2069727) in asthma further confirms the existence of associations differing in direction in two genders. The IFNG association in asthma is particularly intriguing in that the association is with the heterozygous genotype, but in opposite directions in males and 
females, heterozygote advantage in females and heterozygote disadvantage in males $\left(P_{\text {int }}\right.$ $=0.008)^{70}$. Most importantly, this SNP did not show a significant main effect in overall analysis. The same SNP also yielded gender-specific association in childhood ALL risk $^{130}$.

Since most genetic associations are due to the correlation of associated SNPs with gene expression levels, it is of interest to know whether associations differing in directions may be due to sexually dimorphic gene expression ${ }^{26}$. A recent comprehensive survey of HapMap population samples for correlation between SNPs and gene expression levels by gender revealed that $12 \%$ to $15 \%$ of autosomal SNPs function in a genderbiased manner ${ }^{118}$. It was concluded that such signals are likely to be diluted when both genders are analyzed simultaneously. This study highlighted the importance of considering each gender separately in genomic studies to uncover new disease and trait variants. In another study, the effect of serotonin-related gene polymorphisms on the central nervous serotonergic function has showed associations differing in direction between two genders: 5HTTLPR (rs479554, SL6A4; solute carrier family 6) short/short genotype is associated with higher 5-hydroxyindoleacetic acid (5-HIAA) levels in women but with lower levels in men ${ }^{150}$. A gender-genotype interaction was found $\left(\mathrm{P}_{\text {interaction }}=\right.$ 0.04). However, the sample size was small $(\mathrm{n}=165)$. A GWAS meta-analysis identified gender differential in associations with sex hormone-binding globulin levels. The loci 4q13.2-UGT2B15 (rs293428, $\left.\mathrm{P}_{\text {interaction }}=0.003\right), 17 \mathrm{p} 13.1-S H B G\left(\mathrm{rs} 12150660, \mathrm{P}_{\text {interaction }}=\right.$ $0.02)$ and $\mathrm{Xq} 22.3-T D G F 3\left(\mathrm{rs} 1573036, \mathrm{P}_{\text {interaction }}=0.02\right)$ were stronger in men whereas 8q21.12-ZBTB10 (rs440837, $\left.\mathrm{P}_{\text {interaction }}=0.02\right)$ was stronger in women. This study underscored the gender-differentiated effects in complex trait variance ${ }^{151}$. Thus, in 
addition to physiological differences in males and females, there are also differences in the correlations between genetic variants and gene expression levels that suggest feasibility of gender-specific associations we investigated in the present study.

There are also examples of gender-specific associations that are only evident in one gender (male-specific or female-specific). A GWAS on schizophrenia identified a female-specific association with RELN (reelin) gene polymorphism (rs7341475; $\mathrm{P}_{\text {interaction }}$ $\left.=1.8 \times 10^{-4}\right)^{72}$. This polymorphism was further analyzed in four additional populations in the same study, and showed a female-specific association $\left(\mathrm{OR}=1.58, \mathrm{P}=8.8 \times 10^{-7}\right)$ with a significant gender-genotype interaction $\left(\mathrm{P}_{\text {interaction }}=1.6 \times 10^{-5}\right)$. Similarly, LYPLAL1 polymorphism (rs2605100) in waist-hip ratio ${ }^{152}$ also shows a female-specific association. These examples provide the proof of principle that heterogeneity in genetic associations between two genders exist ${ }^{57}$. Thus, it is evident that gender could be considered as an environmental variable in a gene and environment interaction analysis.

In this study, we addressed the gender differences, by treating gender as an environmental factor, to obtain clues about the etiology of childhood ALL pathogenesis. Although the NIH, the Food and Drug Administration (FDA), and the IOM have highly recommended the use of gender-specific analysis, to date gender-specificity remains underappreciated $^{12,153}$. We expected that the proposed study may shed light on the influence of genetic factors in gender related differences in disease pathophysiology. In the absence of known environmental risk factors, genetic markers have the potential to provide etiological clues, and gender-specific analysis is a powerful approach in a disease which shows gender effect ${ }^{68}$. 


\subsection{Age-specific associations}

Like gender, age also plays a significant role in childhood cancer risk ${ }^{22}$. Epidemiological data consistently show a unique age distribution for childhood ALL. Childhood ALL incidence shows highest peak between 1 and 5 years of age, with 72 cases per 1,000,000 per year in the U.S. In contrast, overall incidence rate of childhood ALL is around 23 cases per 1,000,000 per year under age 14, excluding the age group of 1 to 5 years. Overall incidence rate of childhood ALL risk in different age groups are shown in Table 8 . Tumors of the nervous system also show a higher incidence rate in children 0 to 4 years ${ }^{5}$.

Table 8. Descriptive epidemiology of childhood acute lymphoblastic leukemia by age-atdiagnosis

\begin{tabular}{|c|c|c|c|}
\hline Age at diagnosis (y) & Incidence rate* $^{*}$ & Number of cases (n) & \% of cases \\
\hline$<\mathbf{1}$ & 20.0 & 119 & 2.87 \\
\hline $\mathbf{1 - 5}$ & 72.2 & 2091 & 50.41 \\
\hline $\mathbf{6 - 1 0}$ & 30.6 & 868 & 20.93 \\
\hline $\mathbf{1 1 - 1 4}$ & 21.3 & 514 & 12.39 \\
\hline $\mathbf{1 5 - 1 9}$ & 17.8 & 556 & 13.40 \\
\hline
\end{tabular}

*Rate per 1,000,000 per year.

Source: National Cancer Institute, Surveillance, Epidemiology, and End Results ${ }^{5}$

There is significant heterogeneity in the age-of-disease-onset of childhood ALL (Table 8). Around 50\% of childhood ALL occurs between 1 and 5 years. This heterogeneity in age groups suggests that certain factors (genetics or environmental) may influence the severity and early-onset of ALL risk ${ }^{154}$. To understand the relationship between age and childhood ALL risk, direct assessment of the age variation at the population level is important ${ }^{155}$. Just like gender-specific associations may be missed in the overall analysis, age-specific ones should also be unmasked by stratification. An 
association with a small effect size in the overall analysis may become stronger in a certain age group. Statistical interaction analysis is one way to identify age-specific associations or effect modification by age.

\subsection{Genetic basis of age-specific associations}

Cancer in genetically predisposed people occurs at an early age ${ }^{36,156}$. Rare hereditary cancer syndromes, such as Li-Fraumeni syndrome, accelerate the development of several adult cancers ${ }^{134,157}$. Just like the effect of the TP53 (tumor protein 53) mutation in Li-Fraumeni syndrome on age-of-onset, genetic polymorphisms may also accelerate or delay the development of childhood ALL. Any age-specific association, which we aim to find would have been missed in previous studies and would contribute to disease biology in childhood ALL risk. One of the main purposes of GWAS is to find genetic variants that may provide some clues about disease biology related to disease phenotype ${ }^{68}$. Thus, a variant showing a larger effect size for one age group may explain a greater proportion of the population attributable risk estimates, which could improve early detection, treatment and prevention ${ }^{158,159}$.

Several studies have provided empirical evidence for early- or late- onset of diseases, along with severity and their associations with genetic markers. One of the classic examples is late-onset Alzheimer disease (AD) and its association with apolipoprotein $\mathrm{E}(A P O E)$ allele ${ }^{75,76}$. With increasing number of APOE type 4 alleles, mean age-at-onset decreases from 84 to 68 years $^{75}$. APOE codes for the major carrier protein in the brain ${ }^{160}$, and is an important regulator of lipids ${ }^{161}$. It is hypothesized that the presence of APOE type 4 alleles leads to excess amyloid formation in brain $^{162}$. A few 
studies in multiple sclerosis also yielded an association with APOE type 4 alleles and disease severity ${ }^{160,163,164}$. A study on African American females has yielded an earlier age-of-onset of multiple sclerosis (MS) in people with the APOE risk genotype ${ }^{164}$. However, a few of these studies suffer from low statistical power, as the sample size was low $^{163,165}$.

The SNP309 (rs2279744) variant allele, present in the MDM2 (mouse double minute 2) oncogene (cancer causing), is more common in early-onset (AA $=85.2$ months vs $\mathrm{AB}+\mathrm{BB}=70.5$ months $)$ childhood ALL in a multiethnic sample $(\mathrm{P}=0.04)^{154}$. Likewise, the same association with age-at-onset $(\mathrm{P}=0.002)$ is successfully replicated in another childhood ALL samples with gender-specificity in a non-Hispanic white population ${ }^{126}$. Results showed a trend toward an earlier age at diagnosis in females (AA = 59 months vs $\mathrm{AB}=44$ months vs $\mathrm{BB}=32$ months) (133). Studies found that $M D M 2$ expression level is regulated by estrogen signaling in the presence of rs2279744 variant allele $^{134,166,167}$. It has been suggested that rs2279744 variant allele may play a significant role through negatively regulating TP53. TP53 encoded molecules play an important role in genome surveillance (133). MDM2 is a known key negative regulator of TP53 protein, and dysregulation of MDM2 may significantly impact the TP53 functions, and increase tumorigenesis, including leukemia, via apoptotic pathway. Likewise, recent studies have revealed that variant allele of MDM2 rs2279744 can also accelerate tumorigenesis and increase the risk of several cancers, through estrogen-signaling pathway, exclusively in females ${ }^{167}$. Estrogen levels in children are negligible, which suggests in utero exposure of estrogen in childhood ALL risk (133). 
A study on colorectal cancer, in two independent groups, showed that women who carry the variant allele of rs2279744 have a nine year earlier (at 61 years vs 70 years) onset than women who carried the wild-type genotype $(\mathrm{P}=0.001)^{168}$. Results were not statistically significant for men. The authors suggest that estrogen influences variant allele to accelerate colorectal tumor formation in women at a younger age. Likewise, MDM4 polymorphism (rs1563828) had shown an association with an early age-of-onset of estrogen-receptor negative breast cancer in two independent cohorts ${ }^{169}$. Women who possess homozygous variant (TT) develop estrogen-receptor negative breast cancer at an earlier age than homozygous wild-type (CC) and heterozygote (CT) (first cohort: $\mathrm{P}=$ $0.018 \&$ second cohort: $\mathrm{P}=0.006)$. In both cohorts, age-at-diagnosis is accelerated by 5 years (43.8 years vs 48.8 years) and 3.8 years (37.2 years vs 41.0 years), respectively. An human leukocyte antigen (HLA) study on chronic myeloid leukemia (CML) found the age-related effects of HLA-DRB3 and HLA-DRB4 homozygosity ${ }^{170}$. The presence of DRB4 homozygous genotype was associated with risk $(\mathrm{OR}=3.36 ; P=0.01)$ and homozygosity for $\mathrm{DRB} 3$ was protective $(\mathrm{OR}=0.51 ; P=0.007)$. DRB4 homozygosity rate was high and DRB3 homozygosity rate was low in patients with early-onset CML. The age-related changes in their frequencies shown a significant separation $(P=0.02)$ in three age groups (18-32 years, 33-42 years and 43-60 years). The presence of similar associations of PTPN2 (protein tyrosine phosphatase, non-receptor 2) polymorphisms (rs2542151) in $\mathrm{T} 1 \mathrm{D}^{78}$ and hemochromatosis HFE (hemochromatosis) gene variants in colorectal carcinoma ${ }^{171}$ further confirms the genetic effect on age-at-onset of cancers. It is only natural that future association studies should pay attention to these clues. 
A recent study on IKZF1 (IKORAS family zinc finger one) polymorphisms, previously identified as risk markers in childhood $\mathrm{ALL}^{52}$, yielded a significant association between rs4132601 with age at diagnosis $(\mathrm{P}=0.04)^{140}$. The mean age-atonset decreases from 5.58 to 3.83 years $(\mathrm{TT}+\mathrm{TG}=5.58$ vs $\mathrm{GG}=3.83)$ with the possession of two G alleles. The most recent childhood ALL GWAS ${ }^{53}$ compared the variant allele frequency of rs 10821936 between cases that diagnosed ALL before 10 years of age to those that diagnosed at an older age $(>10 \mathrm{y})$. Results showed an association with non-Hispanic whites $(\mathrm{P}=0.02)$ and Hispanics $(\mathrm{P}=0.007)$. This study also yielded a trend for decreasing ORs with increasing age (less than $5 \mathrm{y}, 5$ to $10 \mathrm{y}$ and more than $10 \mathrm{y})$ for the same polymorphism: 2.0 (1.9-2.2), 1.8 (1.6-2.02) and $1.5(1.3-$ 1.7), respectively. Therefore, there are also examples of what we aim to show, namely, age-specific genetic associations in childhood ALL.

Although there are many reports of association with gender and age in cancer susceptibility, they are no more than statistical correlations with yet unknown biological significance and most have not been replicated. These studies need to be confirmed in a much larger population to rule out chance findings. A few of these studies also suffer from multiple comparisons. Most of these associations are identified in a case-control study design, specifically gender-specific associations. A few studies have used convenient samples as a control group, which suggest selection bias. Thus, it is important that controls should be representative of the general population. Future studies should consider the case-only design to avoid problems related to selection bias and insufficient statistical power ${ }^{60}$. Strong effects play an important role in public health interventions or risk profiling ${ }^{158,159}$. We proposed to identify the strongest associations that interact with 
gender and/or age which would have yielded no main effects in the overall analysis in a case-control study. In the present study, we used gender and age as effect modifiers.

Although multiple GWAS have been published in childhood ALL ${ }^{49-54}$, results are consistent but the magnitude of effect is small as in most GWAS. These studies only used an overall analysis approach. Prior to the present study, no GWAS in childhood ALL has used gender- or age-specific analysis. The presence of gender- and age-specific associations underlines the need to conduct a dedicated study on gender- and age-specific analysis to identify the previously missed associations in GWAS and candidate gene studies. The aim of this study was to fill these gaps in knowledge by performing a comprehensive GWAS of genetic susceptibility in childhood ALL to uncover otherwise masked gender- and age-specific associations. 


\section{PROJECT AIMS AND HYPOTHESIS}

Despite multiple published GWAS in childhood ALL, identified genetic susceptibility represents a small fraction, and leaves a large proportion to be defined. We exploited an underutilized design to unravel strong genetic risk markers.

We hypothesized that age and gender differences in childhood ALL risk have a genetic basis, and the effect modification by gender and age can be exploited to unmask yet unknown genetic risk markers.

To assess the validity of this hypothesis, the proposed study had two specific aims:

Specific Aim 1: To examine gender-specific associations that differs in their directions in males and females to gain insight into the pathophysiology of gender differences and childhood ALL pathogenesis.

Specific Aim 2: To examine the relationships of genetic risk markers with age-of-onset of ALL to gain insight into the pathophysiology of age peak in childhood ALL.

The main purpose of this dissertation was to identify gender- and age-specific associations. In case of gender-specific associations we are more interested in the associations which differ in direction (explained in Chapter 2). No study has explored gender-specific associations in childhood ALL GWASs, but all of them only considered the overall group. Likewise, age-specific associations may have been missed in the analyses of the overall sample. For age-specific associations, the peak age (1-5 years) group was compared with the rest of the cases (6-10 years). A time-to-event analysis was also performed for the whole case group. These differences in childhood ALL should be able to provide some clues on disease pathogenesis. The amalgamation of gender- and 
age-specific genome-based knowledge related to disease susceptibility into public health may contribute to disease prevention, health promotion and risk reduction ${ }^{172}$. Bioinformatic analyses were also performed to examine the functionality of the markers that have shown associations. Likewise, empirical data screening was performed to check whether these markers regulate expression of any genes or interfere with the genome biology in any way. The aim of this effort was to identify causal risk factors and gain insight into mechanism of their associations with age-at-onset or gender differential of childhood ALL. 


\section{MATERIALS AND METHODS}

This study was based on two specific aims to identify gender- and age-specific associations with childhood ALL risk. Genome-wide microarray chips were used for all genotyping. Logistic regression, using Plink software, was used to identify gender- and age-specific associations. A time-to-event analysis was performed for the whole group to explore association with age-at-onset.

\subsection{Ethics statement}

Institutional review board (IRB) approval was granted by Florida International University, Office of Research Integrity (IRB-13-0546). A copy of IRB from Baylor College of Medicine (BCM) was also submitted to Office of Research Integrity, Florida International University. All samples received from the primary study site were deidentified, and no one at the FIU site could identify any subject.

\subsection{Research design}

The traditional case-control approach is commonly used in genetic association studies for rare diseases such as childhood ALL. In spite of numerous advantages over other classical epidemiological study designs, case-control study design has several limitations, particularly recruitment of an appropriate control group. The case-only design (explained in Chapter 1) is more efficient than other traditional epidemiological studies at detecting statistical interactions, which was the main focus of this project ${ }^{58}$. Recruitment of cases in a rare disease like childhood ALL is a challenge ${ }^{59}$ and hence, preferring a statistically powerful research design was more practical and is used in the present study. A flow chart explaining whole method section is delineated in Figure 8. 


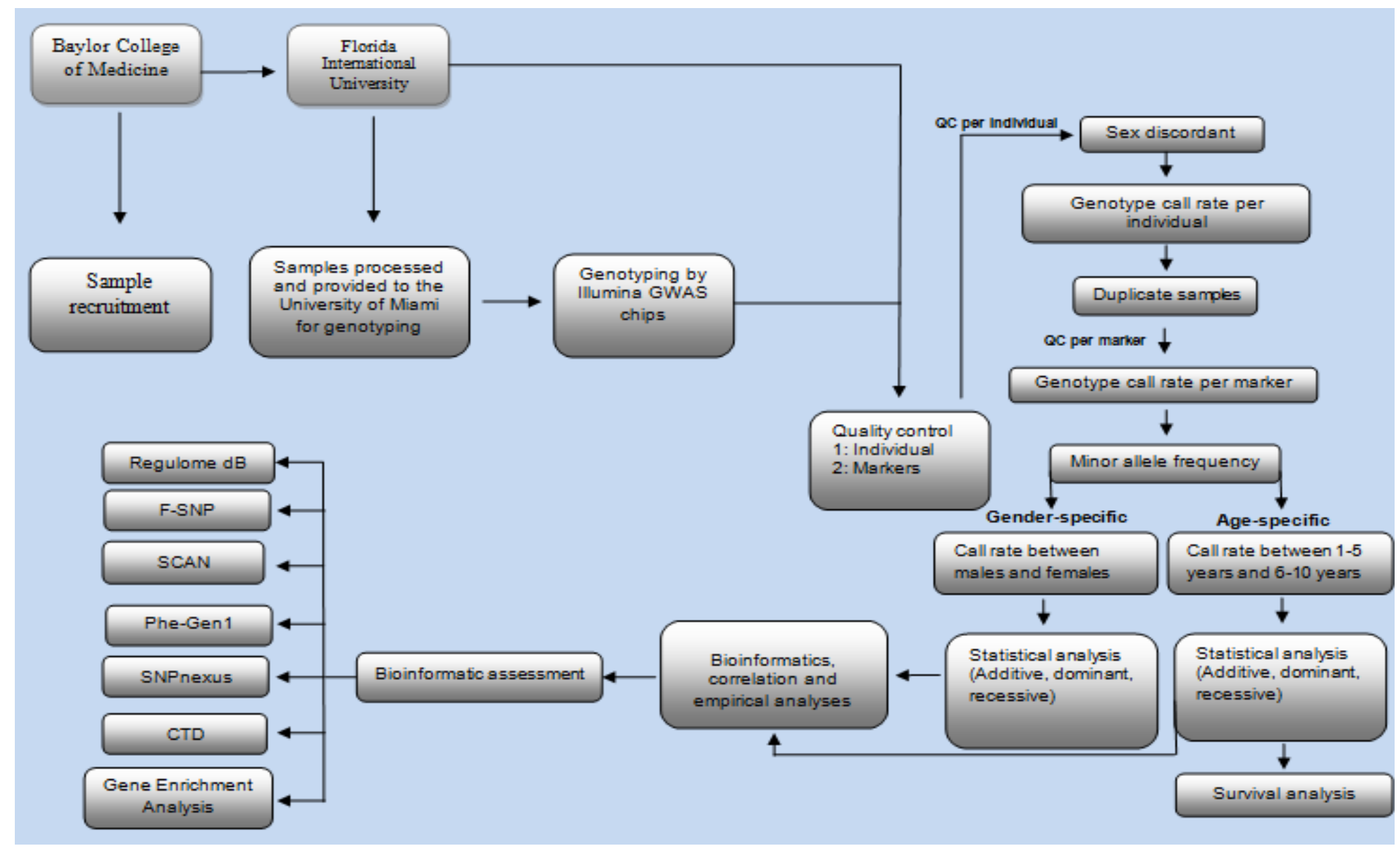

Figure 8. A flowchart explaining method section 


\subsection{Recruitment of study subjects}

A case-control study design was used to recruit the childhood ALL samples in the parent study ${ }^{131}$. In the present study, only cases were used for GWAS to assess the gender- and age-specific analysis. The cases were incident cases of childhood ALL consecutively recruited in Texas Children's Cancer Center (TXCCC), BCM, Pediatric Hematology/Oncology Clinic, Houston, Texas from 2007 to 2012. All newly diagnosed childhood (age 1-18 years) ALL cases and their parents were approached for informed consent and the participation rate was $95 \%$. At the time of enrollment, no case had any other disease. The proposed study used 240 cases for case-only analysis. All of these cases were non-Hispanic whites aged 1 to 10 years. Demographic characteristics of childhood ALL cases collected through questionnaires and are explained in result section (Chapter 5).

\subsubsection{Inclusion criteria}

1- All incident cases diagnosed with acute lymphoblastic leukemia confirmed by immunophenotyping

2- Age 1-18 y in the parent study, but only subjects age 1 to 10 years of age were included in this study nested within the parent study

3- All samples were recruited from TXCCC, BCM, Pediatric Hematology/Oncology Clinic, Houston, Texas

\subsubsection{Exclusion criteria}

1- The exclusion criteria was refusal to participate or diagnosed with any other disease 


\subsection{Statistical power calculation for statistical interaction when gender-specific associations differ in direction (qualitative interaction)}

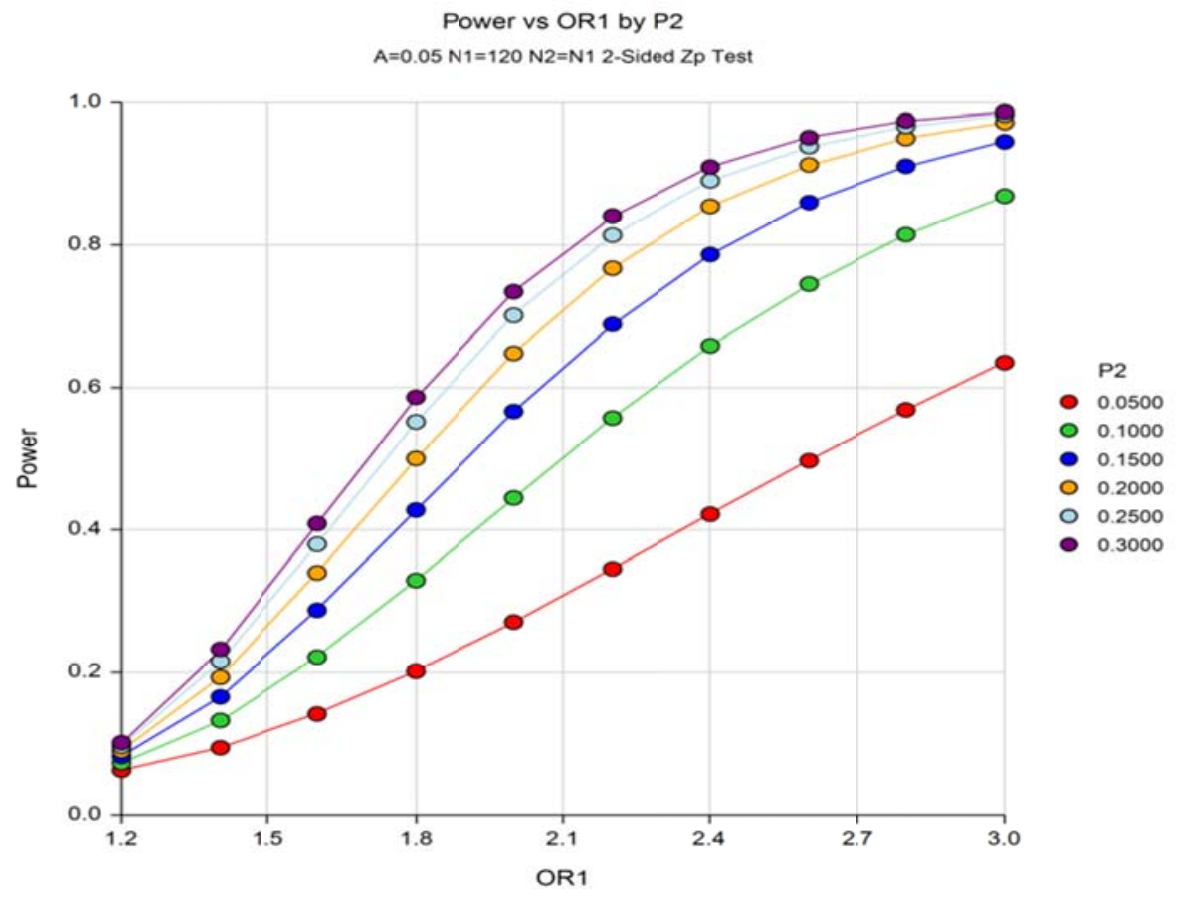

Figure 9. Statistical power calculation for the detection of interactions using the case-only design $\mathrm{X}$-axis is odds ratio, $\mathrm{Y}$-axis is statistical power. Each curve is for a different risk genotype frequency (in the dominant model, the risk genotypes are $\mathrm{AB}+\mathrm{BB}$ where $\mathrm{A}$ is the common and $\mathrm{B}$ is the variant allele). $\mathrm{N}=$ 240

We performed statistical power calculation using power analysis and sample size (PASS) software for the case-only design for various risk genotype frequencies and ORs (Figure $9 \&$ Table 9). For 240 cases, assuming equal number of males and females, to achieve $80 \%$ statistical power for risk genotype frequency of $30 \%$, minimum OR should be 2.2; and for risk genotype frequency of $10 \%$, OR should be at least 2.8 for the association to reach statistical significance. Risk genotype frequency 0.10 corresponds to 0.05 variant allele frequency in the dominant risk model ( $\mathrm{AA}$ vs $\mathrm{AB}+\mathrm{BB})$. 
Table 9. Statistical power calculations for the detection of interactions when associations differ in direction

\begin{tabular}{|c|c|c|c|c|c|c|}
\hline Power & $\begin{array}{c}\text { Sample } \\
\text { size N1 } \\
\text { males }\end{array}$ & $\begin{array}{c}\text { Sample } \\
\text { size N2 } \\
\text { females }\end{array}$ & $\begin{array}{c}\text { Genotype } \\
\text { frequency } \\
\text { in male } \\
\text { cases }\end{array}$ & $\begin{array}{c}\text { Genotype } \\
\text { frequency in } \\
\text { female cases }\end{array}$ & $\begin{array}{c}\text { OR for } \\
\text { interaction }\end{array}$ & $\begin{array}{c}\text { Alpha } \\
(\boldsymbol{\alpha})\end{array}$ \\
\hline 0.8147 & 120 & 120 & 0.24 & 0.10 & 2.8 & 0.05 \\
\hline 0.8686 & 120 & 120 & 0.25 & 0.10 & 3.0 & 0.05 \\
\hline 0.7865 & 120 & 120 & 0.30 & 0.15 & 2.4 & 0.05 \\
\hline 0.8596 & 120 & 120 & 0.31 & 0.15 & 2.6 & 0.05 \\
\hline 0.7673 & 120 & 120 & 0.35 & 0.20 & 2.2 & 0.05 \\
\hline 0.8543 & 120 & 120 & 0.38 & 0.20 & 2.4 & 0.05 \\
\hline 0.8139 & 120 & 120 & 0.42 & 0.25 & 2.2 & 0.05 \\
\hline 0.8407 & 120 & 120 & 0.49 & 0.30 & 2.2 & 0.05 \\
\hline
\end{tabular}

$\mathrm{OR}=$ odds ratio

\subsection{Statistical power calculation for statistical interaction when gender-specific} associations differ in magnitude (quantitative interaction)

We also performed a statistical power calculation for various genotype risk frequencies and effect size for 240 samples (Table 10). However, to achieve $80 \%$ statistical power for risk genotype frequency of $10 \%$ and minimum OR should be 1.59 , and sample size should be 686 in each arm; and for risk genotype frequency $30 \%$ and OR 1.55, sample size should be 356 in each arm. Thus, with a sample size of a total of 240 subjects, we did not have sufficient statistical power to detect quantitative interactions, which we did not aim to detect. 
Table 10. Statistical power calculations for association differing in magnitude

\begin{tabular}{|c|c|c|c|c|c|c|}
\hline Power & $\begin{array}{c}\text { Sample } \\
\text { size N1 } \\
\text { male }\end{array}$ & $\begin{array}{c}\text { Sample } \\
\text { size N2 } \\
\text { female }\end{array}$ & $\begin{array}{c}\text { Genotype } \\
\text { frequency } \\
\text { in male } \\
\text { cases }\end{array}$ & $\begin{array}{c}\text { Genotype } \\
\text { frequency } \\
\text { in female } \\
\text { cases }\end{array}$ & $\begin{array}{c}\text { OR for } \\
\text { interaction }\end{array}$ & $\begin{array}{c}\text { Alpha } \\
(\boldsymbol{\alpha})\end{array}$ \\
\hline 0.22 & 120 & 120 & 0.15 & 0.10 & 1.59 & 0.05 \\
\hline 0.58 & 120 & 120 & 0.20 & 0.10 & 2.25 & 0.05 \\
\hline 0.29 & 120 & 120 & 0.22 & 0.15 & 1.60 & 0.05 \\
\hline 0.49 & 120 & 120 & 0.25 & 0.15 & 1.89 & 0.05 \\
\hline 0.43 & 120 & 120 & 0.30 & 0.20 & 1.71 & 0.05 \\
\hline 0.35 & 120 & 120 & 0.35 & 0.20 & 2.15 & 0.05 \\
\hline 0.39 & 120 & 120 & 0.35 & 0.25 & 1.62 & 0.05 \\
\hline 0.37 & 120 & 120 & 0.40 & 0.30 & 1.55 & 0.05 \\
\hline
\end{tabular}

$\mathrm{OR}=$ odds ratio

\subsection{Statistical power calculation for time-to-event analysis}

We used STATA v13 for statistical power analysis to compare two survival rates (wild-type vs allele positivity). Here we used the dominant genetic risk model (AA as referent vs $\mathrm{AB}+\mathrm{BB}$ as risk genotypes) for statistical power calculation. We calculated statistical power for a range of genotype frequencies. Table 11 represents statistical power for time-to-event analysis for a sample size of 240 based on a range of risk genotype frequencies. The study had $80 \%$ statistical power for the statistical significance threshold of 0.05 , for hazard ratios (HR) ranging between 1.43 and 1.90 for a range of risk genotype frequencies. 
Table 11. Statistical power calculation for time-to-event analysis

\begin{tabular}{|c|c|c|c|}
\hline Power & $\begin{array}{c}\text { Alpha } \\
(\boldsymbol{\alpha})\end{array}$ & $\begin{array}{c}\text { Risk genotype } \\
\text { frequency }\end{array}$ & $\begin{array}{c}\text { Hazard } \\
\text { Ratio }\end{array}$ \\
\hline 0.80 & 0.05 & 0.05 & 1.90 \\
\hline 0.80 & 0.05 & 0.10 & 1.65 \\
\hline 0.80 & 0.05 & 0.15 & 1.55 \\
\hline 0.80 & 0.05 & 0.20 & 1.50 \\
\hline 0.80 & 0.05 & 0.25 & 1.47 \\
\hline 0.80 & 0.05 & 0.30 & 1.45 \\
\hline 0.80 & 0.05 & 0.35 & 1.44 \\
\hline 0.80 & 0.05 & 0.40 & 1.43 \\
\hline
\end{tabular}

\subsection{DNA extraction}

Genomic DNA was extracted from blood samples using the Qiagen Blood Mini Kit (Qiagen, Valencia, California) according to manufacturer's protocol at TXCCC, BCM. Only DNA samples with study IDs without any personal identifiers were received from TXCCC, BCM, Pediatric Hematology/Oncology Clinic, Houston, Texas.

Established childhood ALL risk associations identified in $\mathrm{GWAS}^{51,52}$ have been replicated to validate the parent case-control study sample using TaqMan allelic discrimination assays at FIU $^{131}$.

\subsection{DNA quantification}

DNA samples were quantified via the ND-8000 spectrophotometer and DNA quality was evaluated via gel electrophoresis on a $0.8 \%$ agarose gel at the Hussman Institute for Human Genomics (HIHG), University of Miami. The concentration for all qualified samples was normalized to 50ng/ul and samples were arrayed in Matrix $0.5 \mathrm{ml}$ 2D barcoded tubes in racks of 96 . 


\subsection{Genotyping methods}

Genotypings for 240 childhood ALL cases were achieved by Illumina Infinium HumanCoreExome Chip (Illumina, San Diego, CA, USA). Genotyping was performed at the HIHG, University of Miami on the Illumina iScan array scanner system.

Samples that passed the above exclusion criteria (DNA quantification) were genotyped using Illumina Infinium HumanCoreExome Chip, that interrogates approximately 500,000 markers. The samples were processed according to Illumina Procedures for processing of the Infinium HD assay. In brief, on day one of the procedure 200ng of DNA were denatured and neutralized in preparation for isothermal amplification in an overnight step. On day two, the amplified products were fragmented, precipitated and re-suspended in hybridization buffer. It was then applied to the Infinium HumanCoreExome Chip and the chips were incubated overnight in the Illumina hybridization oven. During this incubation the DNA sample was allowed to anneal to locus-specific 50mers which were covalently attached to beads assembled into microwells on the ExomeChips substrate. On day 3, unhybridized DNA was washed away. Then during a single-base extension of the oligos on the ExomeChips, using the captured DNA as a template, fluorescent labels were incorporated on the ExomeChips and the genotypes were determined. The ExomeChips were then scanned on the Illumina iScan.

The above protocol was automated using the Tecan EVO-1 to further enhance the efficiency and consistency of the assay. Samples were processed in batches of 96. In order to ensure reproducibility of results one quality control DNA sample (CEPH DNA) was repeated in each 96 well plate. Genotype concordance was checked for replicate samples following each genotyping run. Data were extracted by the Illumina ${ }^{\circledR}$ Genome 
Studio software from data files created by the Illumina iscan. No additional pre-Plink QC steps was performed on either the SNPs or the samples.

All post Plink QC steps and association analysis of the genotype data were conducted with the dedicated GWAS analysis software Plink ${ }^{173}$. Plink (http://pngu.mgh.harvard.edu/ purcell/plink) is a free toolset for whole genome association analysis. Most analyses were performed on a Linux-based computer.

\subsection{Quality control}

Genotyping errors have the potential to introduce bias in a genetic association study. QCs are required to remove subjects and genetic markers that do not yield satisfactory results as they may generate false-positive results. 240 samples were genotyped at the UM. All standard GWAS QC steps ${ }^{82,173-177}$ were applied before the analysis. A flowchart of all performed QC steps is given in Figure 10. Results of all QC steps are provided in Chapter 5.

\subsubsection{Per-individual quality control}

Per-individual QC step screens genotypes to identify subjects that may introduce bias if not removed. Poorly genotyped (low call rate) individuals will increase error in the study and may significantly affect the results. All steps performed in this study are explained below. 


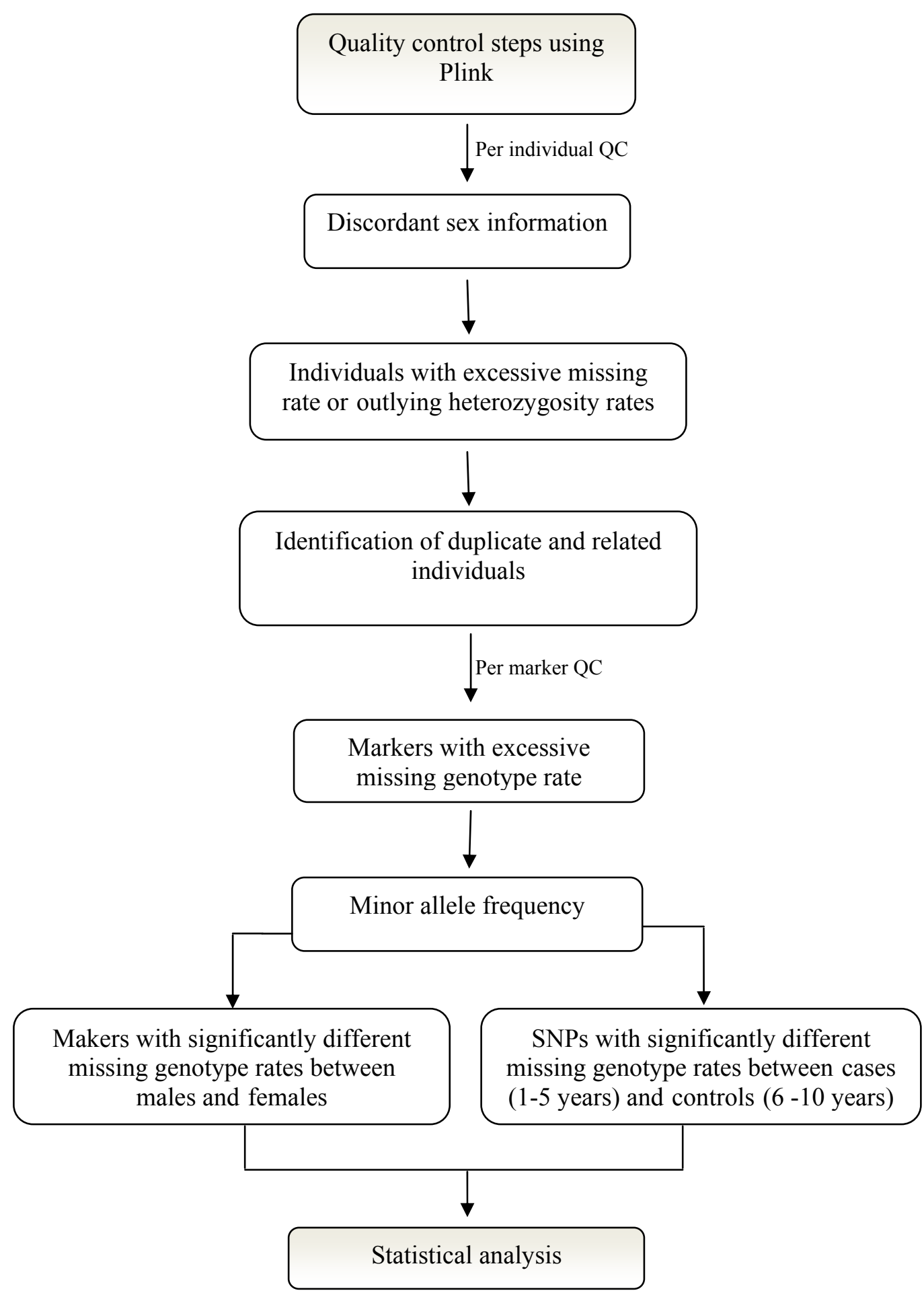

Figure 10. A flow chart of quality control steps used before the gender- and age-specific analysis 


\subsubsection{Identification of individuals with discordant gender information}

This was the first step of QC, performed to identify subjects that have

inconclusive/contradictory gender information ${ }^{82,173}$, as it can lead to spurious associations. In a case-control study, samples that show the wrong gender information are suggested to be excluded from further QC and statistical analysis. Subjects were appropriately recoded or removed, if information was inconclusive, for further analyses.

\subsubsection{Identification of individuals with excessive missing rate or outlying heterozygosity rates}

This QC step was used to identify and exclude individuals with too much missing genotype data and outlying heterozygosity rate. Genotype accuracy and genotype call rate can be significantly affected by variations in DNA quality ${ }^{82}$. A high genotype failure rate and heterozygosity rate suggest poor DNA sample quality. A high heterozygosity rate also suggests DNA contamination. We examined the distribution of missing genotypes in entire data set, and set a threshold of $1 \%$. It means that individuals with more than $1 \%$ missing genotypes would be removed from further QC steps.

\subsubsection{Identification of duplicated or related individuals}

In a population based study, it is important that all samples should be unrelated. Presence of duplicate, first- or second-degree relatives will introduce bias in the study as their genotypes will be overrepresented. This step was used to identify all related and duplicate individuals for removal. A metric (identity by state, IBS) for each pair of individuals was calculated to identify duplicate samples. IBS is defined as, at a locus, two 
individuals who have an identical nucleotide sequence or the same allele. An IBS score 1 suggests relatedness between two samples.

\subsubsection{Per-marker quality control}

Like low quality individual samples, low quality markers can also threaten internal validity of the results. Exclusion of these markers is necessary to avoid spurious associations. The QCs used for marker exclusions are explained below.

\subsubsection{Identification of SNPs with an excessive missing genotyping rate}

Like individuals with a high rate of poor genotypes, we also removed the markers that had high genotyping failure rate. It is important to remove only poorly characterized markers because every removed marker is a potentially missed disease variant ${ }^{82}$. We used a 99\% threshold to remove markers after carefully scrutinizing the data set. It means to include only those SNPs for further analysis which have at least 99\% genotyping call rate. Acceptable genotyping call rates in GWAS are 1 to $5 \%$.

\subsubsection{Removal of markers with a very low minor allele frequency}

In this step of QC, we excluded polymorphisms that had a low minor allele frequency (MAF). These SNPs are mostly non-informative as they show little variation in the sample set being analyzed. Even power to detect an association will also be low. We used a stringent threshold for MAF. SNPs with MAF $\leq 0.03$ in the data set were removed. 


\subsubsection{Identification of SNPs with significantly different missing genotype rates between comparison groups}

Substantial differences in genotyping rates between males and females may confound the result if the difference is caused by DNA sample quality. To reduce confounding, the exclusion of markers with substantially different genotyping rates between these groups is crucial. Likewise, for age-specific association, we also checked differential missing rates between two age groups (1-5 years and 6-10 years). All markers that showed significant difference between two groups were removed from further QC, and gender-and age-specific analyses. We used a threshold of $<0.05$ after scrutinizing the data set.

\subsubsection{Identification of SNPs showing a significant deviation from Hardy- Weinberg equilibrium}

Hardy-Weinberg equilibrium (HWE) refers to genotype frequencies being close to their expected frequencies estimated from allele frequencies. Goodness-of-fit test is used to test the agreement between observed and expected frequencies. In a case-control study, markers which significantly deviate from HWE in controls are usually excluded from analysis since this finding is an indication of genotyping error. Since this is a case-

only study, HWE test has not been used as a QC step to detect genotyping error before the analysis, but markers that have shown associations, were retrospectively assessed for their conformity to HWE in the whole group of subjects and in males and females, or in age groups separately for gross violations that would indicate genotyping errors rather than valid associations. 


\subsection{Genomic control}

The genomic control (GC) is a method to control for population stratification in a genetic association study to reduce type I error ${ }^{176,178,179}$. Population stratification may confound results, if not adjusted. A population in a genetic association study may arise from two distinct sub-populations which may differ in their genetic ancestry. The presence of systematic differences in allele frequencies between these two subpopulations may confound the result. There are thousands of neutral loci, known as genomic controls used to correct for population stratification in GWASs. The GC first estimates an inflation factor $(\lambda)$, then adjust all of the test statistics downward by $\lambda$. Inflation factor $\lambda>1$ indicates population substructure and/or genotyping error. Plink was used to adjust results for genomic control. In this study, only non-Hispanic whites were used for gender- and age-specific associations. We still adjusted results for GC to avoid any confounding by population stratification.

\subsection{Permutation test}

Permutation testing, also known as randomization testing, is a type of statistical significance test that is not influenced by multiple comparisons ${ }^{180}$. Rearrangement of the phenotype status, such as case-control status, of each sample is the first step of this test. The second step is to compute association analysis after each rearrangement of the casecontrol status. This whole procedure is performed by predefined number of times, 10,000 times in the present study. Test statistics is calculated for actual data set, and each permuted data set for predefined number of times. The procedure keeps the $\mathrm{P}$ value after

each permutation. At the end, it compares the distribution of permuted $\mathrm{P}$ values with the 
$\mathrm{P}$ value yielded by the original data set. This comparison estimates the proportion of chance findings if the study was repeated predefined number of times under the null hypothesis. These results are free from multiple comparison issues, as each permutation on each SNP assesses the role of chance, and can be performed on thousand of SNPs independently. In this study, for gender-specific association analysis, the rearrangement of phenotype status involved males and females. Likewise, for age-specific analysis, the shuffle was performed between age group 1-5 years (coded as one) and 6-10 years (coded as two).

\subsection{Data analysis}

All data analyses were performed using Plink and Stata v13 (StataCorp, College Station, TX). For the comparison of continuous variables, results were expressed as means and standard deviations (SD) and student t-test (for means) was performed. Dichotomous or categorical variables were summarized as a table with counts and percentages for comparison, and Pearson's chi-squared and fisher exact test were performed.

Unconditional logistic regression was used to calculate OR and 95\% CI for gender- and age-specific analyses. The main interest was detecting the genotype frequency differences between males and females cases. We used logistic regression after coding males and females as " 2 " and " 1 " as if they were cases and controls, respectively. Similarly, in age-specific analysis, we compared different genotype frequencies between two age groups (1-5 years vs 6-10 years). Logistic regression was also used for agespecific analysis after coding age group 1-5 years as "2" and 6-10 years as "1". 
Definition of variables for gender-specific and age-specific are summarized in respective tables (Tables 12 and 13). We used GC to adjust for population heterogeneity ${ }^{178,}{ }^{179}$, however, all of these samples were non-Hispanic whites.

Table 12. Summary of variables for gender-specific association

\begin{tabular}{|l|l|l|}
\hline Variables & Classification & Measurement \\
\hline Outcome variable & & \\
\hline Gender & Dichotomous & 2 (male) or 1 (Female) \\
\hline Explanatory variable & & \\
\hline Genotype & Categorical & AA, AB, BB \\
\hline Confounding variable & & \\
\hline Age & Continuous & years \\
\hline
\end{tabular}

Table 13. Summary of variables for age-specific association

\begin{tabular}{|l|l|l|}
\hline Variables & Classification & Measurement \\
\hline Outcome variable & & \\
\hline Age & Dichotomous & $2(1$ to 5 y) or $1(6$ to 10 y) \\
\hline Explanatory variable & & \\
\hline Genotype & Categorical & AA, AB, BB \\
\hline Confounding variable & & \\
\hline Gender & Categorical & 1 (Females) or 2 (Male) \\
\hline
\end{tabular}

\subsection{Genetic models for different association tests}

There are several genetic models (Figure 11) used in the analysis of a genetic association study ${ }^{181}$. These genetic models have similarities to the models used in 


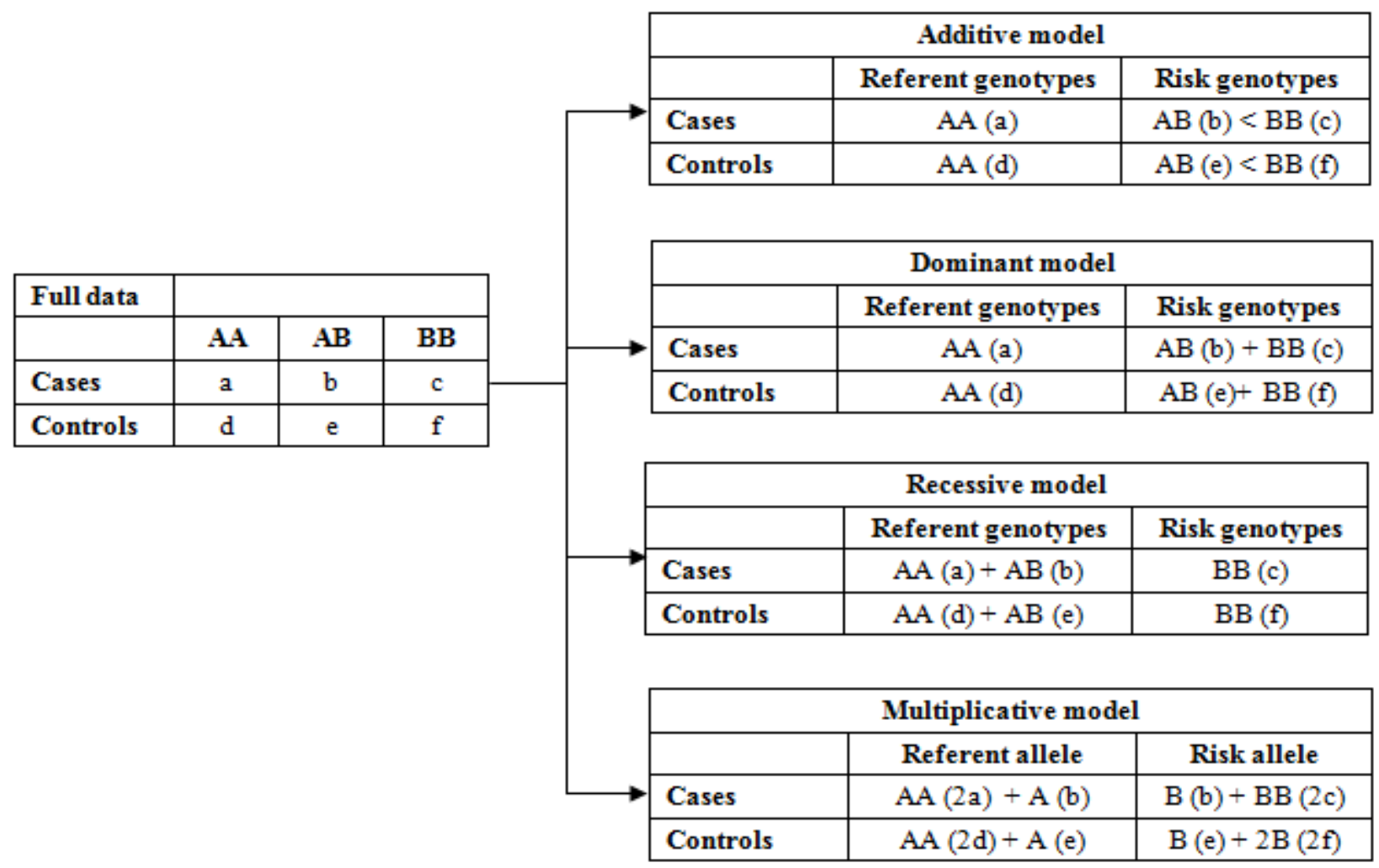

Figure 11. Common genetic risk models use in a genetic association study

Here, A and B are two alleles of a SNP. AA is a wild-type, AB is a heterozygote and BB is a variant genotype.

Adapted from: Lewis CM, Knight J. Introduction to genetic association studies. Cold Spring Harb Protoc 2012; 2012(3): 297-306 
environmental epidemiological studies. An additive genetic model was used gender- and age-specific association analyses. X-chromosome analysis was based on multiplicative genetic model. Time-to-event analysis genetic models were based on Kaplan-Meier plot. The counts for three genotypes of a SNP can be analyzed by a 2 x 3 contingency table. For a SNP with allele A and B, genotypes will be AA (wild-type), AB (heterozygote) and BB (variant).

\subsubsection{Additive model}

The most common genetic model used in the analysis of an association study is known as an additive model (Figure 11). This model is tested by Cochran-Armitage trend test which assesses gene-dosage effect. This model assumes that having two copies of minor allele (BB) will have double the effect of having a single copy of minor allele (AB). This genetic model is very similar to a basic epidemiological model (no smoking vs 5 cigarettes/day vs 10 cigarettes/day). This test has one degree of freedom. These data can be analyzed by logistic regression in which the genotypes are represented as 0 (AA), $1(\mathrm{AB})$ and $2(\mathrm{BB})$.

\subsubsection{Dominant model}

This model (Figure 11) suggests that even a single copy of the variant allele has an effect on phenotype. We categorized individuals into two groups (AA vs $\mathrm{AB}+\mathrm{BB}$ ) based on whether they have at least one minor allele B (either AB or BB) or no copies of minor allele (AA). It is a $2 \times 2$ table test with one degree of freedom. This model is also very similar to basic epidemiological model, for example presence or absence of exposure. 


\subsubsection{Recessive model}

In the recessive genetic risk model, two copies of variant allele (BB) is associated with risk (Figure 11). In other words, heterozygosity $(\mathrm{AB})$ is not associated with risk. Here, we categorized individuals in two groups ( $\mathrm{AA}+\mathrm{AB}$ vs $\mathrm{BB})$. It is tested in a $2 \times 2$ table test with 1 degree of freedom.

\subsubsection{Multiplicative model}

In case of X-chromosome analysis, genotype-based models are not practical for statistical analysis. Females have two copies of X chromosomes and males have one copy of $\mathrm{X}$ chromosome. The most appropriate test is allelic association test. In allelic association test (Figure 11) the unit of test is the allele (A and B) rather than individual, as each individual contributes two alleles for autosomal chromosomes. Allele counts for $\mathrm{X}$-chromosome are obtained in a different way for $\mathrm{X}$ chromosome polymorphisms, and are given below.

In case of $\mathrm{X}$ chromosome analysis

Females have a pair of $\mathrm{X}$ chromosome means two copies of alleles (AA or AB or BB).

$\mathrm{AA}=$ two A alleles

$\mathrm{AB}=$ one $\mathrm{A}$ and one $\mathrm{B}$ allele

$\mathrm{BB}=$ two $\mathrm{B}$ alleles

Males have one $\mathrm{X}$ chromosome, means one copy of allele (A or B).

$\mathrm{A}=1$ allele

$\mathrm{B}=1$ allele 


\subsection{Time-to-event analysis}

Time-to-event analysis (survival analysis) was performed on each SNP that has shown an association in gender- and age-specific analysis. The top 100 results based on the $\mathrm{P}$ value and the effect size were used for time-to-event analysis. Kaplan-Meier plots were used to generate the survival curves between two or three groups, cases possessing different genotypes (wild-type vs heterozygote vs homozygous variant). It also provided clues for appropriate genetic model (additive, dominant or recessive) to test the statistical significance between three or two curves (depending on the genetic risk model).

Log-rank test was used to test the statistical difference between survival times (time to having leukemia) between two (dominant or recessive model) or three (additive model) groups. Cox-proportion hazard model were used to calculate the $\mathrm{HR}^{182,183}$. Definition of variables for survival analysis is summarized in Table 14.

Table 14. Summary of variables for survival analysis

\begin{tabular}{|l|l|l|}
\hline Variables & Classification & Measurement \\
\hline Outcome variable & & \\
\hline Time-to-event & Continuous & years \\
\hline Explanatory variable & & \\
\hline Genotype & Categorical & AA, AB, BB \\
\hline Confounding variable & & \\
\hline Gender & Categorical & 1 (Female) or 2 (Male) \\
\hline
\end{tabular}


All statistical tests were two-tailed and threshold for statistical significance was 0.05. We performed a permutation test for gender- and age-specific associations which is not influenced by multiple comparisons.

\subsection{Bioinformatic and empirical screening of data for functional annotation of associated markers}

To assess the functionality of identified gender- and age-specific associated SNPs, several bioinformatic tools were used. The main purpose was to examine whether identified variants are causative or proxy markers. RegulomeDB ${ }^{184}$ (http://regulomedb.org/) and Functional Single Nucleotide Polymorphism (F-SNP) ${ }^{185}$ (http://compbio.cs.queensu.ca/F-SNP/) were the two main databases used for this purpose. RegulomeDB predicts a score for a variant from 1 to 7 . A score of 1 signifies the highest functionality of the variant, and 7 the least functional variant. To assess functionality, RegulomeDB uses high throughput, computational predictions and other sources such as experimental results. F-SNP database generates a score between 0 and 1 . A score of 1 suggests a high functional score and 0 suggests least functional score. This database incorporates information obtained from 16 other bioinformatic tools and the functional effects of SNPs. These databases identify and focus on SNPs with potential harmful effect on human health.

Two SNPs, close to each other, on the same chromosome may be statistically correlated. These highly correlated SNPs will not provide any additional information for statistical analysis. It means that SNP1 and SNP2 will give the same information, such as effect size (OR) and P value. These highly correlated SNPs, included in the GWAS chip, 
were identified through Plink analysis, to avoid redundancy. All top SNPs that show correlations are reported in the appendix.

Most of the time, genetic association studies yield associations with nearby correlated SNPs, known as indirect associations. The second step of correlation analysis was to identify primary causal SNP, which was not included in the GWAS chip. We generated a list of SNPs, using HaploReg (http://www.broadinstitute.org/mammals/haploreg/haploreg.php), for all gender- and agespecific risk variants, which were correlated. Bioinformatic and empirical analyses were performed to identify potentially causal SNPs.

We also used empirical analysis using SNP and Copy Number Annotation (SCAN) database ${ }^{186}$ (http://www.scandb.org/newinterface/index.html). For each SNP, SCAN database provides information from experimental data on gene expression regulation. Gene expression is a quantitative trait and an important intermediate phenotype that link the genetic polymorphisms and phenotype (here, ALL). Any genetic variant that regulates gene expression levels of mRNA and protein is known as an expression quantitative trait locus (eQTL). The correlations between SNPs and gene expressions ultimately modify disease susceptibility. Thus, eQTL analysis was used to evaluate whether the identified gender- and age-specific variants influence expression levels of genes. We searched SCAN database and only explored the eQTL effects of identified variants in lymphoblastoid cell lines (LCLs) in the Caucasian population. The $P$ value was $\leq 1 \times 10^{-5}$.

Phenotype-Genotype Integrator (PheGenI) database (http://www.ncbi.nlm.nih.gov/gap/phegeni) was used to increase chances of findings of 
susceptible candidate genes ${ }^{187}$. The PheGenI, merges National Human Genome Research Institute (NHGRI) GWAS catalog data with several other databases housed at the National Center for Biotechnology Information. SNPnexus database (http://www.snpnexus.org/) was used to retrieve additional information, such as genomic mapping (using $\mathrm{dbSNP})$, gene/protein consequences, effect on protein function, regulatory elements, and phenotype and disease association ${ }^{188}$.

Gene enrichment analysis, also known as pathway-based analysis, is a powerful approach to identify disease causal genes from genomic, proteomic and bioinformatics dataset. It looks an identified gene list, here gender- and age-specific genes, and finds out whether any group of genes belonging to the same pathway are overrepresented in the list. We used the Gene Ontoloy Consortium (http://geneontology.org/) for gene enrichment analysis ${ }^{189}$. Bonferroni correction was used for multiple comparisons. The Comparative Toxicogenomics Database (CTD) was used to identify chemical-genedisease relationships ${ }^{190}$. The $\mathrm{CTD}$ also provides insight into chemical-gene, gene-disease and chemical-disease interactions. It nearly contains 15 million toxicogenomic relationships. 


\section{RESULTS}

The project, which investigated gender- and age-specific genetic associations with childhood ALL risk using a GWAS design has yielded positive results as presented in this chapter.

\subsection{Quality control results}

The main aim of QC was to minimize potential bias and error by excluding samples and markers, which failed at QC steps. These excluded samples were also compared with samples analyzed in this study to check whether their exclusion introduced any bias. All steps of QC, explained in Chapter 4, and their results, are explained below. Four samples were excluded after agarose gel DNA quantification based on lower DNA concentration.

\subsubsection{Results from discordant gender information}

The first step of QC was used to check the self-reported gender information of each individual using genetic data. Plink uses $\mathrm{X}$ chromosome data to determine gender empirically. This calculation is based on the difference between $\mathrm{X}$ chromosome numbers

in males (one $\mathrm{X}$ chromosome) and females (a pair of X chromosomes) ${ }^{82}$. Males cannot be heterozygous $(\mathrm{AB})$ for any marker. Genotype-calling algorithm for this QC step counts heterozygote as a missing genotype. A female marked as a male in the data set will show a large amount of missing data. This step of QC gives a score between 0 and 1 . In an ideal situation, we expect homozygosity rate one for males and $<0.2$ for a female subject. However, a score of $\leq 0.2$ suggests a female, and a score of $\geq 0.8$ suggests a male. Scores 
between 0.2 and 0.8 suggest ambiguity in gender information. In the case of discrepancy between self-reported sex and genetically determined sex, the genetically determined sex was used in the analysis. Seven males $(\geq 0.8)$ and six females $(\leq 0.2)$ were re-coded on re-examination of their gender in the original dataset. Four samples scored between 0.2 and 0.8, and were removed from further QC steps due to ambiguous gender information which could not be verified. However, most of these gender discordant samples also failed further QC steps suggesting that the ambiguity was due to poor DNA sample quality. Inclusion of individuals, with discordant gender information, could have yielded spurious associations, and could have hampered the first aim of the study. The idea with gender QC is not just to get the genders right, but also to rule out any sample handling/labeling problems.

\subsubsection{Results from individuals genotype and heterozygosity rates}

The aim of this QC step was to identify individuals who had low genotype call (genotyping) rates and higher heterozygosity rates. Low genotype call rates and higher heterozygosity rates are indicative of poor DNA quality. Aberrant genotype calling would increase errors in the study, and those samples should be excluded. We excluded twenty samples that had the genotype failure rate $\geq 1 \%$ and /or the heterozygosity rate outside of

\pm 3 standard deviations from the mean. A graph showing how the heterozygosity rate and genotype call rate correlate is presented in the appendix (Figure 1). These excluded samples were further analyzed to check if there is any substantial difference between them and the rest of the samples (explained in 5.2). 


\subsubsection{Results from duplicates and related samples}

This step of QC was used to identify duplicates and samples from closely related individuals to avoid overrepresentation of their genotypes which would introduce bias in the study. Plink calculates a metric for each pair of individuals and generates a score between 0 and 1. A score of one suggests the presence of a duplicate sample in the data set. A total of five samples were excluded from further analysis as they scored more than $98 \%$ ( $>0.98)$ with another sample. All other samples had very low scores $(<0.14)$. Among these five samples, one DNA sample was repeated in each 96 well plate (total three), and one sample was used a duplicate. Our results yielded one more sample as a duplicate.

\subsubsection{Results from genotyping efficiency}

This step of QC was used to examine the markers genotyping efficiency. Marker genotype efficiency (genotyping rate per marker) indicates marker quality. This QC step was used to identify and exclude poorly genotyped markers. These markers are likely to increase false associations, if not excluded. We used a $\geq 99 \%$ threshold to remove markers after carefully scrutinizing the data set. This suggests that markers with $1 \%$ or more missing data were excluded. A total of 4,710 out of 538,449 markers failed this QC step, and were removed from further analysis.

\subsubsection{Results from minor allele frequency filter}

Removal of markers based on a low allele frequency rate was the next step of the QC. Although this step is a part of QC, it does not mean that quality of markers was low. These SNPs are mostly non-informative as they show little variation in the sample set 
being analyzed. The statistical power to detect an association will also be low. The main aim of this step was not to hamper the quality of the subsequent analysis. A stringent threshold $(\leq 0.03)$ was used to filter rare alleles. A total of 259,565 markers had minor allele frequency $\leq 0.03$ and were excluded from further analysis.

\subsubsection{Results from genotyping rates between two groups}

The next step of QC was to identify markers that show significant differences in genotyping rates between two groups (males vs females and age groups 1-5 y vs 6-10 y). To minimize confounding, markers that showed significant difference in their genotyping rates between two groups were excluded from further analysis. We used a threshold of $>$ 0.05 after critically scrutinizing the data set. A total of 569 SNPs had significantly different $(>0.05)$ genotyping rates between two genders and were removed. Likewise, 217 SNPs showed a significant difference between the two age groups and were excluded from analysis.

\subsubsection{Results from Hardy-Weinberg equilibrium}

In this study, HWE was used retrospectively to assess their conformity in the whole group of subjects in gender- and age-specific analyses. A significant deviation from HWE in healthy controls suggests genotyping error, and must be excluded from analysis. After critically examining the data set we used a threshold of $\mathrm{P}<10^{-3}$. None of the top one hundred polymorphisms in gender- and age-specific associations showed a significant deviation from HWE in gender- and age-specific analyses.

Genotype call rates were greater than $99.9 \%$ for all childhood ALL cases after all QC steps were implemented for gender- and age-specific association analyses. This 
translates into $<0.01$ missing data points. Figure 12 explains a brief summary of all QC steps and results. Differences between samples that were excluded from final analysis and samples that were retained are reported in the next section.

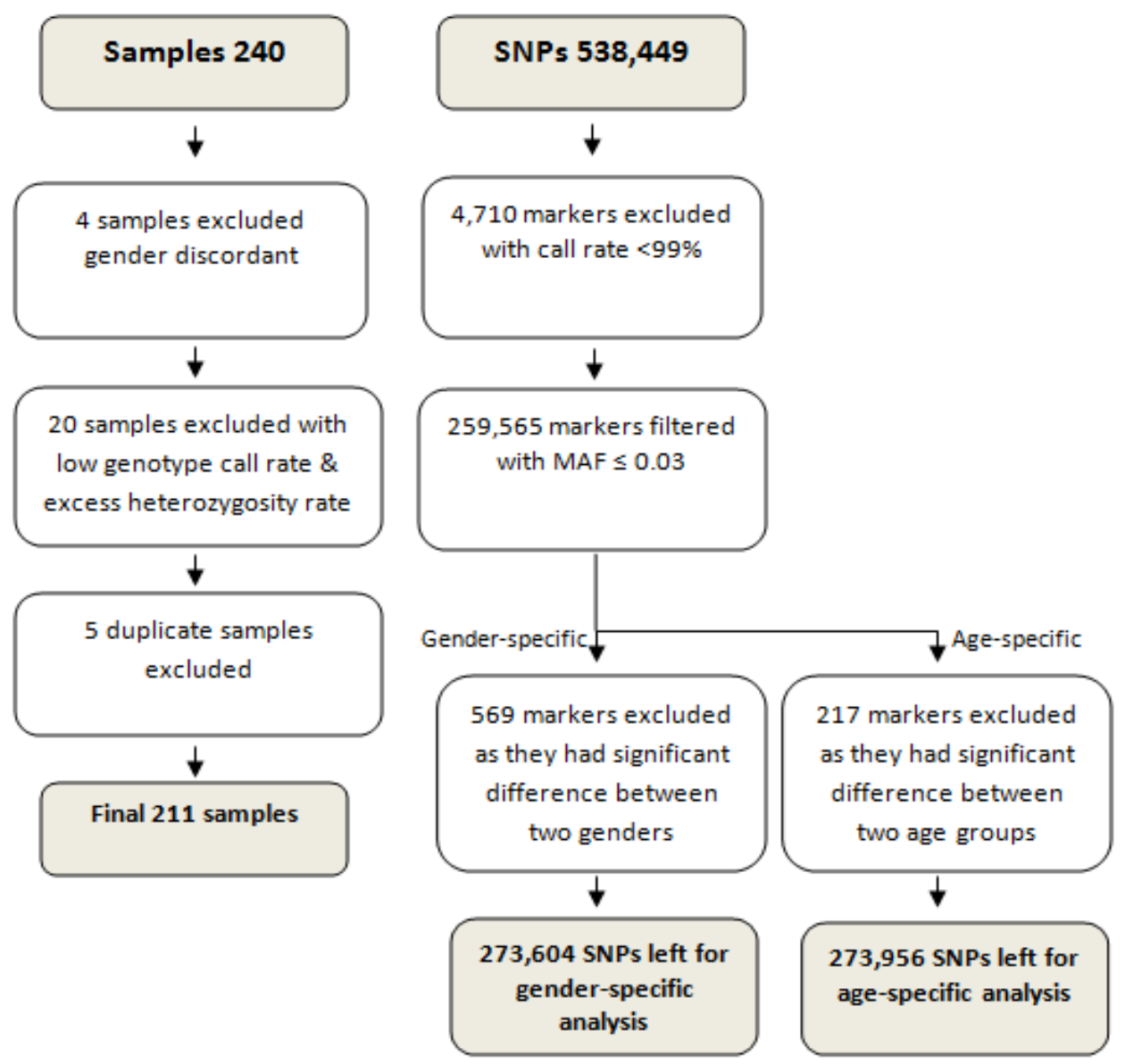

Figure 12. Quality control steps, and samples and markers exclusion 


\subsection{Characteristics of childhood ALL study subjects}

All of these samples identified themselves as non-Hispanic white. The final sample included 117 males (55.5\%) and 94 females (45.5\%) (Table 17). This study was restricted to age group 1-10 years. There was no statistically significant difference $(\mathrm{P}=$ $0.10)$ between two genders in their age distribution (Table 18). The mean ages in males (4.44 years) and females $(4.70$ years $)$ were not different $(\mathrm{P}=0.45)$. For age-specific analysis, 143 samples $(67.8 \%)$ were age $1-5$ years and 68 samples $(32.2 \%)$ were age $6-10$ years. Male-to-female ratios were not different $(\mathrm{P}=0.42)$ between the two age groups. Individuals excluded after QC were compared with individuals retained for final analysis to check any substantial difference between the two groups. A total of 37 individuals were excluded from final analysis, including individuals who were excluded before genotyping. There were no significant differences between the mean ages $(\mathrm{P}=0.67$; samples excluded: 4.35 years vs samples included: 4.56 years) or male-to-female ratios between the two groups $(\mathrm{P}=0.45)$. Thus, there appeared to be no substantial difference between the subgroup of excluded samples and the rest of the sample to implicate any selection bias. 
Table 15. Characteristics of childhood ALL cases

\begin{tabular}{|c|c|}
\hline Gender & \\
\hline Male & $117(55.5 \%)$ \\
\hline Female & $94(45.5 \%)$ \\
\hline Age (year) & \\
\hline Mean (SD) & $4.56(2.46)$ \\
\hline Median (IQR) & $4(3-6)$ \\
\hline Age (year) & \\
\hline 1 & $17(8.10 \%)$ \\
\hline 2 & $28(13.30 \%)$ \\
\hline 3 & $45(21.30 \%)$ \\
\hline 4 & $29(13.70 \%)$ \\
\hline 5 & $24(11.40 \%)$ \\
\hline 6 & $17(8.10 \%)$ \\
\hline 7 & $17(8.10 \%)$ \\
\hline 8 & $15(7.10 \%)$ \\
\hline 9 & $13(6.20 \%)$ \\
\hline 10 & $6(2.80 \%)$ \\
\hline Age groups & \\
\hline $1-5$ year & $143(67.8 \%)$ \\
\hline $6-10$ year & $68(32.2 \%)$ \\
\hline Ethnicity & Non-Hispan \\
\hline
\end{tabular}

SD: standard deviation, IQR: Inter quartile range, ALL: acute lymphoblastic leukemia 
Table 16. Characteristics of male and female childhood acute lymphoblastic leukemia cases

\begin{tabular}{|c|c|c|c|}
\hline Age/Gender & Male & Female & P value \\
\hline $\mathbf{1}$ & $13(11.1 \%)$ & $4(4.3 \%)$ & \\
\hline $\mathbf{2}$ & $12(10.3 \%)$ & $16(17.0 \%)$ & \\
\hline $\mathbf{3}$ & $31(26.5 \%)$ & $14(14.9 \%)$ & \\
\hline $\mathbf{5}$ & $15(12.8 \%)$ & $14(14.9 \%)$ & \\
\hline $\mathbf{6}$ & $11(9.4 \%)$ & $13(13.8 \%)$ & \\
\hline $\mathbf{7}$ & $6(5.1 \%)$ & $11(11.7 \%)$ & \\
\hline $\mathbf{8}$ & $10(8.5 \%)$ & $7(7.4 \%)$ & \\
\hline $\mathbf{9}$ & $6(5.1 \%)$ & $9(9.6 \%)$ & \\
\hline $\mathbf{1 0}$ & $9(7.7 \%)$ & $4(4.3 \%)$ & \\
\hline \multicolumn{4}{|r|}{} \\
\hline Age (year) & $4(3.4 \%)$ & $2(2.1 \%)$ & $\mathbf{0 . 1 0}$ \\
\hline Mean (SD) & $4.44(2.56)$ & $4.70(2.33)$ & $\mathbf{0 . 4 5}$ \\
\hline Median (IQR) & $4(3-6)$ & $4(3-6)$ & $\mathbf{0 . 3 1}$ \\
\hline
\end{tabular}

SD: standard deviation, IQR: Inter quartile range

\subsection{Results of gender- and age-specific associations}

As routinely done in GWAS, the additive genetic risk model was used for genderand age-specific analyses using logistic regression. In addition, permutation testing $(10,000$ permutations; $\mathrm{P} \leq 0.05$ for statistical significance) was performed for top one hundred results, using Plink, to eliminate false-positives due to chance. These results are, therefore, free from multiple comparison issues as the permutation test assesses the role of chance on each SNP. Thus, no other statistical approach was used to correct these 
results for multiple comparisons. Permutation $\mathrm{P}$ values alongside the logistic regression results are reported in all tables.

\subsubsection{Results of gender-specific associations}

A. Q-Q plot for gender-specific association unadjusted and before QC
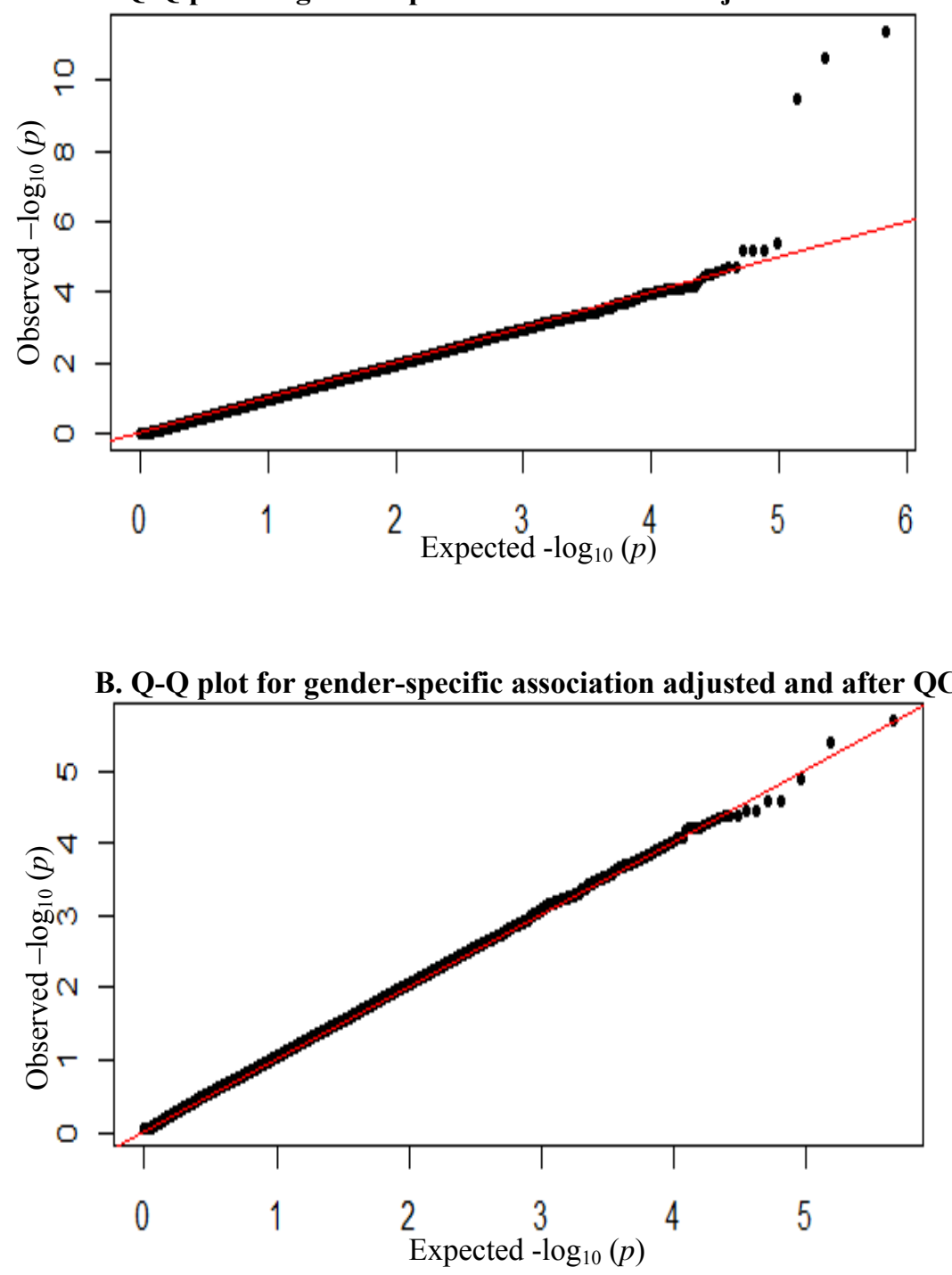

Figure 13. Q-Q plot for gender-specific association analysis for childhood ALL risk

The $\mathrm{x}$-axis represents expected $P$ value on logarithmic scale (genomic control), and the $\mathrm{y}$-axis represents observed $P$ value on logarithmic scale (GC);. A: Q-Q plot is unadjusted for GC. B: Q-Q plot is adjusted for genomic control. 
The additive genetic risk model was used for analysis of genotype distribution differences between male and female cases for each SNP, and results were adjusted for potential population stratification using the genomic control (GC) method. A quantilequantile (Q-Q) plot (Figure 13) was used to explore systematic errors, and to generate a visual representation of observed $\mathrm{P}$ values of GWAS results against expected $\mathrm{P}$ values. The genomic inflation factor for gender-specific analysis was one. A score of one indicates no confounding by population stratification. Manhattan plot was generated using the results for the additive model (Figure 14).

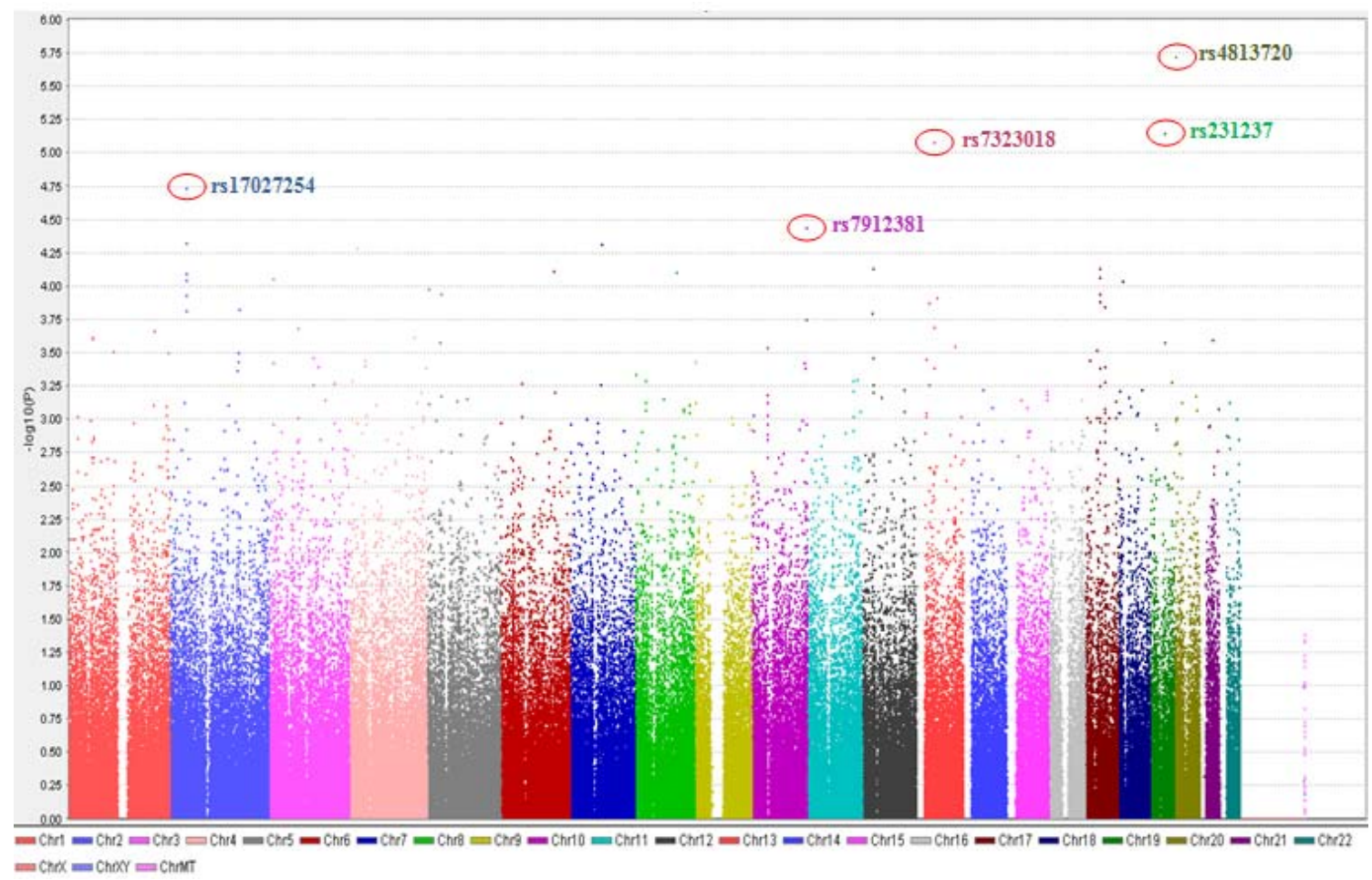

Figure 14. Manhattan plot for gender-specific association analysis for childhood acute lymphoblastic leukemia risk

The $\mathrm{x}$-axis represents position on each chromosome and the $\mathrm{y}$-axis shows the $\mathrm{P}$ value on logarithm scale from the additive genetic model. 
The Manhattan plot visualizes the distribution of association results across the genome and with their statistical significance. Here, the X-axis represents SNPs position on each chromosome and the $\mathrm{Y}$-axis shows $\mathrm{P}$ values on a log scale.

When results were ranked by their $P$ values, the smallest $P$ value was $1.9 \times 10^{-6}$ $\left(\right.$ RASSF2, rs4813720, $\left.\mathrm{OR}_{\text {interaction }}=0.29\right)($ Table 17). Appendix Table 2 shows results for the top one hundred gender-specific associations based on the lowest $\mathrm{P}$ value. Altogether 18 SNPs showed associations with a $P$ value of $<1 \times 10^{-4}$ (Figure 15). Of the top 100 ranking associations, the maximum number of gender-specific associations was found on chromosome $17(\mathrm{n}=15)$. Several genomic loci, such as 17q12 (ACACA) and 2p22.1, yielded multiple associations. However, some of these associations (rs7583193 and rs 17027254; rs1899286, rs6732320 and rs11687208; rs8081866, rs2542653, rs2542660 and rs12948120) were due to correlated SNPs (Appendix Table 3). 
Table 17. SNPs showed gender-specific associations in childhood ALL risk at the significance level $<1 \times 10^{-4}$

\begin{tabular}{|c|c|c|c|c|c|c|c|c|c|}
\hline \multirow[b]{2}{*}{ SNP } & \multirow[b]{2}{*}{ Gene/region } & \multirow[b]{2}{*}{$\mathrm{Chr}^{\dagger}$} & \multirow[b]{2}{*}{$\begin{array}{l}\text { Minor } \\
\text { allele }\end{array}$} & \multirow[b]{2}{*}{ Feature } & \multicolumn{2}{|c|}{$\begin{array}{l}\text { Minor allele } \\
\text { frequency }\end{array}$} & \multirow[b]{2}{*}{$\mathrm{OR}_{\text {allele }}(95 \% \mathrm{CI}) *$} & \multirow[b]{2}{*}{ P value** } & \multirow[b]{2}{*}{$\begin{array}{c}\text { P value } \\
(\text { permutation })^{\dagger \dagger}\end{array}$} \\
\hline & & & & & Males & Females & & & \\
\hline rs4813720 & RASSF2 & 20 & $\mathrm{~A}$ & 5upstream & 0.23 & 0.47 & $0.29(0.17-0.48)$ & $1.90 \times 10^{-06}$ & $1.0 \times 10^{-4}$ \\
\hline rs231237 & HSPB6 & 19 & A & 5upstream & 0.42 & 0.21 & $3.00(1.86-4.85)$ & $7.15 \times 10^{-06}$ & $1.0 \times 10^{-4}$ \\
\hline rs7323018 & $13 q 14.1$ & 13 & $\mathrm{G}$ & intergenic & 0.17 & 0.36 & $0.31(0.18-0.52)$ & $8.40 \times 10^{-06}$ & $1.0 \times 10^{-4}$ \\
\hline rs17027254 & $2 \mathrm{p} 22$ & 2 & $\mathrm{~T}$ & intergenic & 0.19 & 0.39 & $0.37(0.23-0.58)$ & $1.86 \times 10^{-05}$ & $1.0 \times 10^{-4}$ \\
\hline rs7912381 & $10 \mathrm{q} 26.3$ & 10 & $\mathrm{G}$ & intergenic & 0.39 & 0.21 & $2.78(1.71-4.51)$ & $3.68 \times 10^{-05}$ & $1.0 \times 10^{-4}$ \\
\hline rs11687208 & $2 \mathrm{p} 22$ & 2 & $\mathrm{C}$ & intergenic & 0.21 & 0.40 & $0.40(0.26-0.62)$ & $4.81 \times 10^{-05}$ & $1.0 \times 10^{-4}$ \\
\hline rs798292 & MAGI2 & 7 & G & intronic & 0.16 & 0.35 & $0.38(0.24-0.61)$ & $4.88 \times 10^{-05}$ & $1.5 \times 10^{-4}$ \\
\hline rs13107783 & $4 p 15.3$ & 4 & $\mathrm{C}$ & intergenic & 0.32 & 0.52 & $0.40(0.26-0.62)$ & $5.16 \times 10^{-05}$ & $2.0 \times 10^{-4}$ \\
\hline rs4470624 & $4 p 15.3$ & 4 & $\mathrm{~T}$ & intergenic & 0.32 & 0.52 & $0.40(0.25-0.62)$ & $5.16 \times 10^{-05}$ & $2.0 \times 10^{-4}$ \\
\hline rs1849374 & $12 \mathrm{p} 11.2$ & 12 & A & intergenic & 0.44 & 0.25 & $2.48(1.58-3.88)$ & $7.35 \times 10^{-05}$ & $2.0 \times 10^{-4}$ \\
\hline rs12948120 & ACACA & 17 & $\mathrm{C}$ & intronic & 0.29 & 0.49 & $0.44(0.29-0.66)$ & $7.46 \times 10^{-05}$ & $3.0 \times 10^{-4}$ \\
\hline rs6904762 & $6 q 23$ & 6 & G & intergenic & 0.27 & 0.46 & $0.41(0.26-0.64)$ & $1.00 \times 10^{-05}$ & $1.0 \times 10^{-4}$ \\
\hline rs506389 & $8 \mathrm{q} 22.3$ & 8 & $\mathrm{~A}$ & intergenic & 0.28 & 0.11 & $2.99(1.74-5.15)$ & $7.98 \times 10^{-05}$ & $2.0 \times 10^{-4}$ \\
\hline rs6732320 & $2 \mathrm{p} 22$ & 2 & $\mathrm{~T}$ & intergenic & 0.2 & 0.38 & $0.41(0.26-0.64)$ & $8.08 \times 10^{-05}$ & $3.0 \times 10^{-4}$ \\
\hline rs8081866 & ACACA & 17 & $\mathrm{~T}$ & intronic & 0.28 & 0.48 & $0.45(0.30-0.67)$ & $8.64 \times 10^{-05}$ & $3.0 \times 10^{-4}$ \\
\hline rs349714 & $3 \mathrm{p} 25$ & 3 & $\mathrm{G}$ & intergenic & 0.44 & 0.26 & $2.43(1.56-3.80)$ & $8.76 \times 10^{-05}$ & $2.0 \times 10^{-4}$ \\
\hline rs7583193 & $2 \mathrm{p} 22$ & 2 & $\mathrm{G}$ & intergenic & 0.19 & 0.38 & $0.41(0.26-0.64)$ & $9.09 \times 10^{-05}$ & $1.0 \times 10^{-4}$ \\
\hline
\end{tabular}

$* \mathrm{OR}_{\text {interaction }}$ per allele $\left(\mathrm{OR}_{\text {allele }}\right)$ for the additive model; ${ }^{* *} \mathrm{P}$ value adjusted for genomic control; ${ }^{\dagger} \mathrm{Chr}=\mathrm{Chromosome}, \mathrm{SNP}=$ single nucleotide polymorphism; ${ }^{\dagger} \mathrm{P}$ value permutation $=$ point wise $\mathrm{P}$ value from maxT permutation analysis after 10,000 permutation 


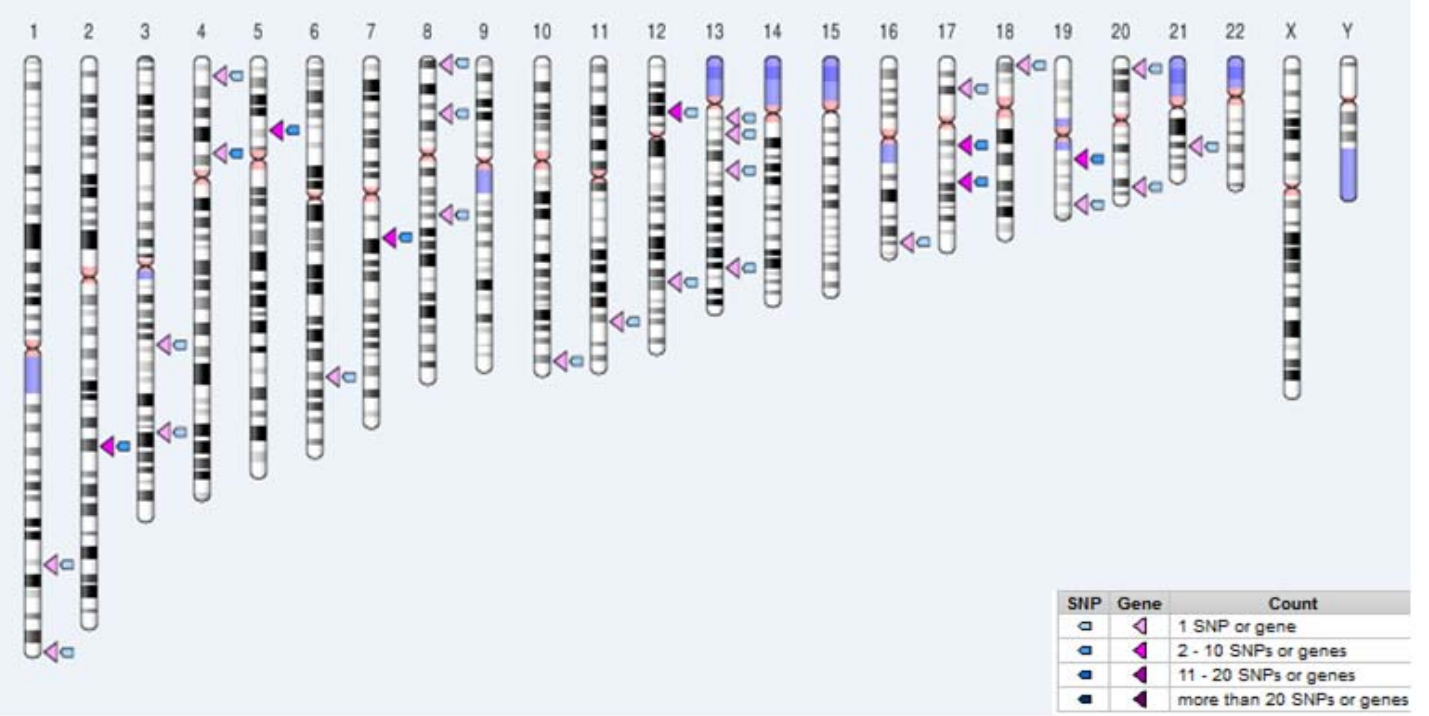

Figure 15. Genome view of single nucleotide polymorphisms or associated genes in gender-specific associations based on $P$ values

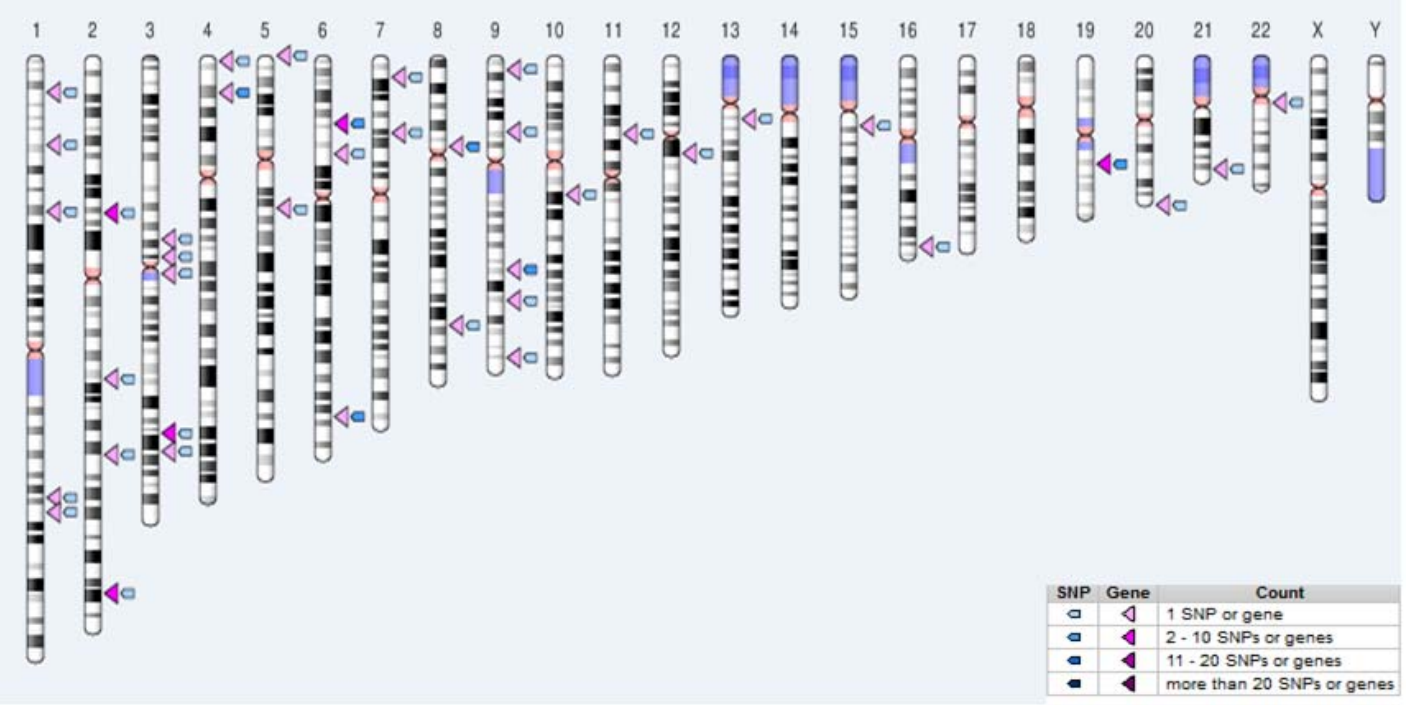

Figure 16. Genome view of single nucleotide polymorphisms or associated genes in gender-specific associations based on effect size 
When we ranked the results by their effect size, the largest effect size (OR) was 15.7 (HLA-DQA1; rs 12722042) (Table 18). Strength of the association was remarkably high $\left(\mathrm{OR}_{\text {interaction }} \geq 10.0\right)$ for nine SNPs. The top two associations were from the HLADQA1 gene with SNPs (rs12722042 and 12722039), which were correlated.

Chromosome 2 and chromosome 6 yielded the maximum number of associations, 15 and 13, respectively (Figure 16). There were sets of SNPs from these regions that were correlated with one another (Appendix Table 4). ADAM28, at chromosome 8, showed the maximum number of associations (rs1 1992342, rs7829965, rs36041430), however, correlated. Around $50 \%$ of identified variants were from intergenic regions (regions between genes). Appendix Table 5 shows results for the top one hundred gender-specific associations based on the highest effect size. 
Table 18. SNPs showed gender-specific associations in childhood ALL risk with the highest effect sizes $(O R>10 ; P$ value $<0.05)$

\begin{tabular}{|c|c|c|c|c|c|c|c|c|c|}
\hline \multirow[b]{2}{*}{ SNP } & \multirow[b]{2}{*}{ Gene/region } & \multirow[b]{2}{*}{$\mathbf{C h r}^{\dagger}$} & \multirow[b]{2}{*}{$\begin{array}{c}\text { Minor } \\
\text { allele }\end{array}$} & \multirow[b]{2}{*}{ Feature } & \multicolumn{2}{|c|}{$\begin{array}{l}\text { Minor allele } \\
\text { frequency }\end{array}$} & \multirow[b]{2}{*}{$O R_{\text {allele }}(95 \% \mathrm{CI}) *$} & \multirow[b]{2}{*}{ P value*** } & \multirow[b]{2}{*}{$\begin{array}{c}\text { P value } \\
\text { (permutation) }\end{array}$} \\
\hline & & & & & Males & Females & & & \\
\hline rs12722042 & HLA-DQA1 & 6 & $\mathrm{G}$ & coding & 0.07 & 0.005 & $15.68(2.03-121.2)$ & 0.008 & 0.001 \\
\hline rs12722039 & HLA-DQA1 & 6 & $\mathrm{~A}$ & coding & 0.07 & 0.005 & $14.87(1.94-114.0)$ & 0.009 & 0.002 \\
\hline rs11992342 & ADAM28 & 8 & $\mathrm{~T}$ & intron & 0.07 & 0.005 & $14.73(1.92-113.9)$ & 0.01 & 0.001 \\
\hline rs80040922 & UMODL1 & 21 & $\mathrm{~A}$ & coding & 0.06 & 0.005 & $13.68(1.77-105.6)$ & 0.01 & 0.004 \\
\hline rs61753605 & LOC100996481 & 6 & $\mathrm{C}$ & coding & 0.06 & 0.005 & $13.38(1.73-103.3)$ & 0.01 & 0.004 \\
\hline rs35665085 & CECR5 & 22 & $\mathrm{~A}$ & coding & 0.06 & 0.005 & $12.64(1.63-98.00)$ & 0.02 & 0.006 \\
\hline rs143021649 & CNTN3 & 3 & $\mathrm{~T}$ & coding & 0.06 & 0.005 & $11.62(1.49-90.58)$ & 0.02 & 0.009 \\
\hline rs6795524 & PROS1 & 3 & $\mathrm{G}$ & intron & 0.05 & 0.005 & $10.63(1.36-83.31)$ & 0.02 & 0.006 \\
\hline rs10003468 & $4 q 28.1$ & 4 & $\mathrm{C}$ & intergenic & 0.05 & 0.005 & $10.63(1.36-83.31)$ & 0.02 & 0.005 \\
\hline
\end{tabular}

$* \mathrm{OR}_{\text {interaction }}$ per allele $\left(\mathrm{OR}_{\text {allele }}\right)$ for the additive model; $* * P$ value adjusted for genomic control; ${ }^{\dagger} \mathrm{Chr}=\mathrm{Chromosome} ; \mathrm{SNP}=$ single nucleotide polymorphism; ${ }^{\dagger} \mathrm{P}$ value permutation $=$ point wise $\mathrm{P}$ value from maxT permutation analysis after 10,000 permutation 
Top ranking results based on the effect size showed associations with more missense (resulting in amino acid changes) variations than the results based on the $\mathrm{P}$ values (29 vs 2) (Appendix Table 6 and Table7). Thus, OR-based top ranking results were enriched for missense and/or highly functional SNPs. These missense SNP associations with high effect sizes and obvious functional effects on protein structure suggest that most of these variants may be causative rather than indirect associations. We performed bioinformatic analyses to examine functionality of all gender-specific markers. The aim was to check whether these markers were direct or indirect associations with childhood ALL risk. Two main databases (RegulomeDB and F-SNP, explained in Chapter 4) were used to obtain functional score of markers. Bioinformatic analyses of these SNPs yielded high levels of functionality by at least one algorithm for 13 SNPs based on the P value, and for 25 SNPs based on the effect size (Appendix Table 6 and Table7). Two SNPs, rs8106959 and rs739924, scored 1f for results based on the P value. The SNP rs739924 also scored one for F-SNP. Likewise, one SNP (rs6929434) scored 1b and four SNPs (rs12722042, rs3847262, rs11751765 and rs130069) scored $\geq 0.75$ by FSNP database. We also performed bioinformatic analysis of SNPs which were not included in the GWAS chip but were highly correlated with gender-specific markers (Appendix Table 6 and Table7). Results are only provided if a correlated SNP showed a much higher score, by bioinformatic analysis, than associated SNP. Several associated SNPs showed correlation with multiple functional variants. The SNP rs9913430 scored 1a, as the most credible 
functional variants for results based on the P value. Moreover, two SNPs scored 1d (rs9314355 and rs7224979) and two SNPs (rs10413852 and rs1275051) scored 1f for results based on the P values. For results based on the effect size one SNP (rs12349952) scored $1 \mathrm{f}$. We also performed empirical data screening to complement bioinformatics assessment (Appendix Table 8). When we screened existing experimental data, using SCAN, only eleven SNPs (results based on the P value) and one SNP (results based on the effect size) showed substantial effects on regulation of several genes in the nonHispanic population.

The SNPnexus database provided information from the GWAS catalogue and the Catalogue of Somatic Mutations in Cancer (COSMIC). A total of five SNPs (rs552976, rs563694, rs560887, rs12940887 and rs9305777) previously showed associations with other diseases and traits (Appendix Table 9). Interestingly three SNPs (rs552976, rs563694 and rs560887) were located in the same gene (G6PC2). All of these three associated SNPs were associated with glucose-related traits. The SNP rs12940887 showed an association with blood pressure level and rs9305777 was associated with platelet aggregation. The COSMIC database showed three SNPs (rs61753605, rs35693261 and rs4645656), which may cause functional changes that are advantageous for cancer occurrence and/or progression. The SNP rs61753605 showed associations with two cancer sites (breast cancer and prostate cancer). However, the SNP, rs35693261, may increase the risk of intestinal cancer and rs 4645656 may increase the chance of stomach cancer. These are somatic mutations. Somatic mutations in cancer cells may be drivers (causing or helping progression of cancer) or passengers (just the results of random events). All somatic mutations were associations from the highest effect sizes from 
gender-specific associations. A total of seven SNPs were located in the transcription factor binding sites. The transcription factor binding sites are mostly located within the promoter region of genes, and may initiate gene function. Thus, SNPs located in these regions may have more regulatory functional effects.

The CTD was used to examine associated genes for their interactions with environmental exposures, such as carcinogens. A list of gender-specific genes accompanied by their interacting chemicals and appropriate references can be found in the appendix (Table $10 \&$ Table 11). Nearly all genes have shown substantial potential with chemical interactions in humans and animal models. The most common environmental interactions were with vehicle emissions (benzene, 2,6-dinitrotoluene, ozone), drugs (acetaminophen), tobacco smoke (benzo(a)pyrene, benzo(a)pyrene diol epoxide, nicotine), poly aromatic hydrocarbons (PAH) (benzo(a)pyrene), food cooked on the barbecue (benzo(a)pyrene, benzo(a)pyrene diol epoxide), pesticides (fonofos, parathion, terbufos, methoxychlor, chlorpyrifos), polychlorinated biphenyls, bisphenol A, formaldehyde and hormones (estrogen, progesterone and testosterone). Most of these environmental chemicals are ubiquitous and classified as class 1 (benzene, benzo(a)pyrene, benzo(a)pyrene diol epoxide tetrachlorodibenzodioxin, aflatoxin B1, formaldehyde, asbestos, arsenic, nicotine and estradiol) and class 2 (progesterone, polychlorinated biphenyls, ethanol) carcinogens by International Agency for Research on Cancer (IARC) ${ }^{191}$. Some of these chemicals also act as teratogens, such as alcohol, tobacco smoke, lead, mercury, benzene, benzo(a)pyrene, estradiol, progesterone, arsenic, pesticides and formaldehyde, and may affect disease susceptibility in utero. There are few examples of gene-environment interaction and childhood ALL risk. Most of these 
environmental exposures have not yet been properly evaluated in childhood ALL risk. Several studies have found that exposure to these environmental pollutants, such as pesticides, benzene, tobacco smoke, formaldehyde and polychlorinated biphenyls, specifically during pregnancy, can increase risk of hematopoietic cancers including

childhood ALL ${ }^{192-196}$, however, these results are not robust and confirmed ones. Geneenvironment interaction with gender-specificity may unravel some environmental risk factors and increased childhood ALL risk. Studies have also unraveled gender-specificity in chemical exposures and metabolisms ${ }^{197-200}$.

\subsubsection{Results of age-specific associations}

As in gender-specific associations, the additive genetic model was used to compare genotype distributions between two age groups, and results were adjusted for population stratification using the genomic control method. A Q-Q plot (Figure 17) was used to explore systematic errors in the age-specific associations. The adjusted results again yielded a genomic inflation factor one for age-specific association. Again, inflation factor one ruling out confounding by cryptic population stratification. 
A: Q-Q plot for age-specific analysis unadjusted and before QC
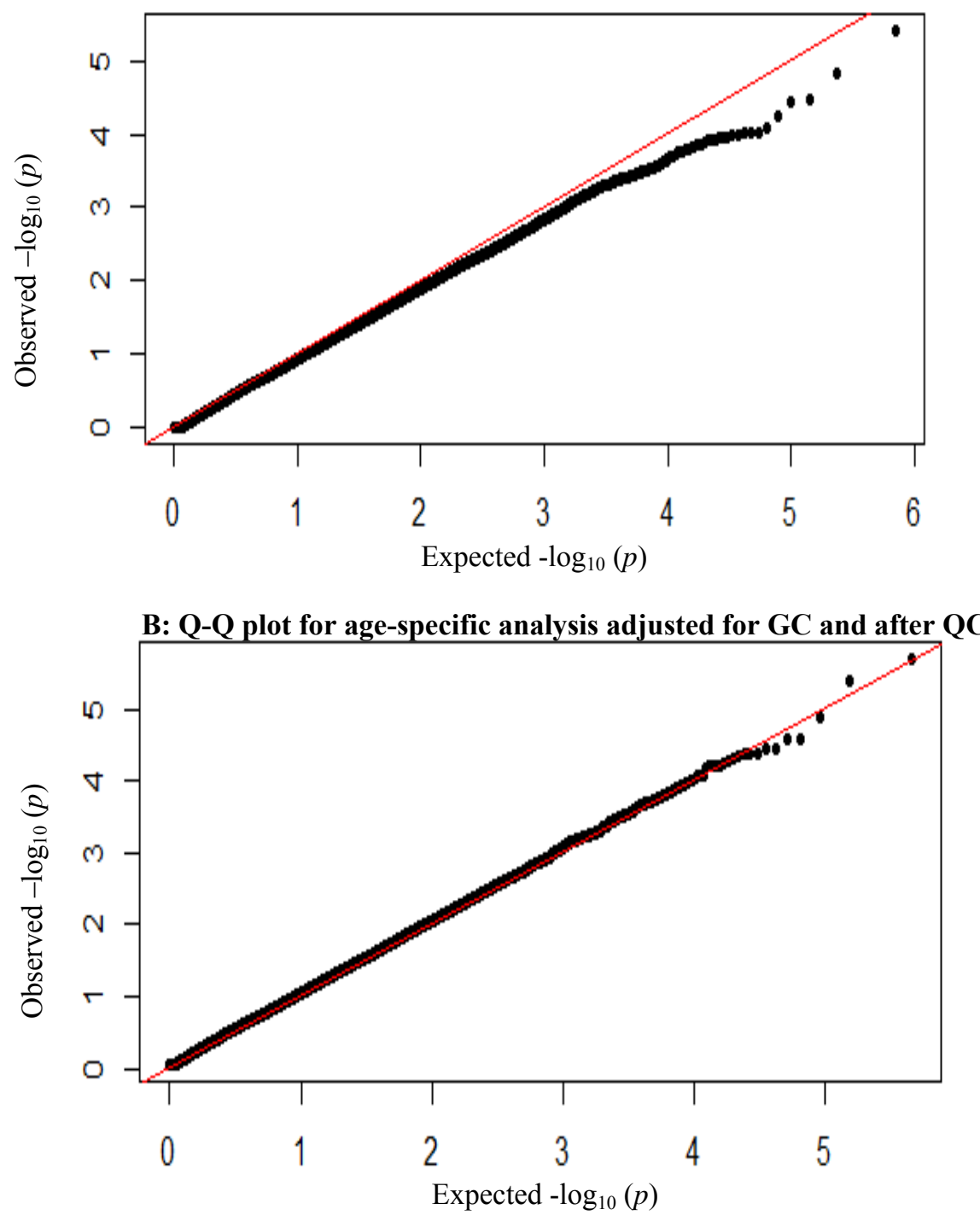

Figure 17. Q-Q plot for age-specific association analysis for childhood ALL risk

The $\mathrm{X}$-axis represents expected $\mathrm{P}$ value on logarithm scale (genomic control), and the $\mathrm{y}$-axis represents observed P value on logarithm scale (GC); A: Q-Q plot unadjusted for GC and observed before QC B: Q-Q plot adjusted for genomic control and observed after QC. 
A total of ten SNPs showed statistically highly significant age-specific associations $\left(P\right.$ value $\left.\leq 1 \times 10^{-4}\right)$, and the lowest $\mathrm{P}$ value was $9.84 \times 10^{-6}(\mathrm{SOX} 5$; rs 10505918) (Table 19). Among the top one hundred associations (Appendix Table 12), chromosome 14 yielded the maximum number of results (Figure 18), some SNPs being in correlation with one another (Appendix Table 13). All three polymorphisms (rs8021355, rs7144565 and rs2274068) at RALGAPA1 (chromosome 11) were perfectly correlated. Several other genomic loci, such as 2p24.3, 5q14.2, 16q22.2 and 21q21.3, also showed multiple associations, but few variants (rs377621 and rs16979806; rs6739633 and rs 1521244) at these loci were correlated.

When results were ranked by their effect size, the largest effect size was 12.5 (DIO1, rs17109582) (Table 20 and Appendix Table 14). A total of 4 SNPs have shown high effect sizes $\left(\mathrm{OR}_{\text {interaction }}>10\right.$, all with $\left.\mathrm{P}<0.05\right)$. The maximum number of agespecific association (12 associations) was observed at chromosome 2 and chromosome 6 (Figure 19). Some of these associations were correlated (Appendix Table 15). 
Table 19. SNPs showed age-specific associations in childhood ALL risk at the significance level $<1 \times 10^{-4}$

\begin{tabular}{|c|c|c|c|c|c|c|c|c|c|}
\hline \multirow[b]{2}{*}{ SNP } & \multirow[b]{2}{*}{ Gene/region } & \multirow[b]{2}{*}{$\mathrm{Chr}^{\dagger}$} & \multirow[b]{2}{*}{$\begin{array}{l}\text { Minor } \\
\text { allele }\end{array}$} & \multirow[b]{2}{*}{ Feature } & \multicolumn{2}{|c|}{$\begin{array}{l}\text { Minor allele } \\
\text { frequency }\end{array}$} & \multirow[b]{2}{*}{$\mathrm{OR}_{\text {allele }}(95 \% \mathrm{CI})^{*}$} & \multirow[b]{2}{*}{$P$ value $* *$} & \multirow[b]{2}{*}{$\begin{array}{c}\text { P value } \\
\text { (permutation) }\end{array}$} \\
\hline & & & & & $1-5 y$ & 6-10 y & & & \\
\hline rs10505918 & SOX5 & 12 & A & intron & 0.42 & 0.19 & $3.15(1.90-5.24)$ & $9.84 \times 10^{-06}$ & $1 \times 10^{-4}$ \\
\hline rs11997355 & $8 \mathrm{q} 21.11$ & 8 & $\mathrm{~T}$ & intergenic & 0.05 & 0.19 & $0.22(0.11-0.44)$ & $2.74 \times 10^{-05}$ & $1 \times 10^{-4}$ \\
\hline rs2388773 & $16 \mathrm{q} 12.2$ & 16 & $\mathrm{C}$ & intergenic & 0.14 & 0.35 & $0.35(0.21-0.57)$ & $3.78 \times 10^{-05}$ & $1 \times 10^{-4}$ \\
\hline rs665159 & CHRM3 & 1 & $\mathrm{~T}$ & intron & 0.55 & 0.33 & $2.46(1.58-3.84)$ & $7.42 \times 10^{-05}$ & $2 \times 10^{-4}$ \\
\hline rs3954950 & $9 \mathrm{p} 24.2$ & 9 & $\mathrm{~T}$ & intergenic & 0.24 & 0.43 & $0.38(0.24-0.62)$ & $7.77 \times 10^{-05}$ & $1 \times 10^{-4}$ \\
\hline rs7818844 & $8 \mathrm{p} 12$ & 8 & $\mathrm{~T}$ & intergenic & 0.40 & 0.60 & $0.38(0.23-0.61)$ & $7.86 \times 10^{-05}$ & $1 \times 10^{-4}$ \\
\hline rs6972158 & NPSR1 & 7 & G & intron & 0.34 & 0.14 & $3.03(1.74-5.25)$ & $8.30 \times 10^{-05}$ & $1 \times 10^{-4}$ \\
\hline rs7320982 & $8 \mathrm{p} 12$ & 13 & $\mathrm{C}$ & intergenic & 0.19 & 0.38 & $0.39(0.24-0.62)$ & $8.33 \times 10^{-05}$ & $1 \times 10^{-4}$ \\
\hline rs7583258 & $2 \mathrm{q} 22.3$ & 2 & $\mathrm{G}$ & intergenic & 0.09 & 0.24 & $0.29(0.16-0.54)$ & $8.79 \times 10^{-05}$ & $2 \times 10^{-4}$ \\
\hline rs1882591 & $16 \mathrm{q} 12.2$ & 16 & $\mathrm{G}$ & intergenic & 0.22 & 0.42 & $0.42(0.27-0.64)$ & $9.60 \times 10^{-05}$ & $2 \times 10^{-4}$ \\
\hline
\end{tabular}

$* \mathrm{OR}_{\text {interaction }}$ per allele $\left(\mathrm{OR}_{\text {allele }}\right)$ for the additive model; ${ }^{* *} P$ value adjusted for genomic control; ${ }^{\dagger} \mathrm{Chr}=$ Chromosome, $\mathrm{SNP}=$ single nucleotide polymorphism; ${ }^{\dagger} \mathrm{P}$ value permutation $=$ point wise $\mathrm{P}$ value from maxT permutation analysis after 10,000 permutation 


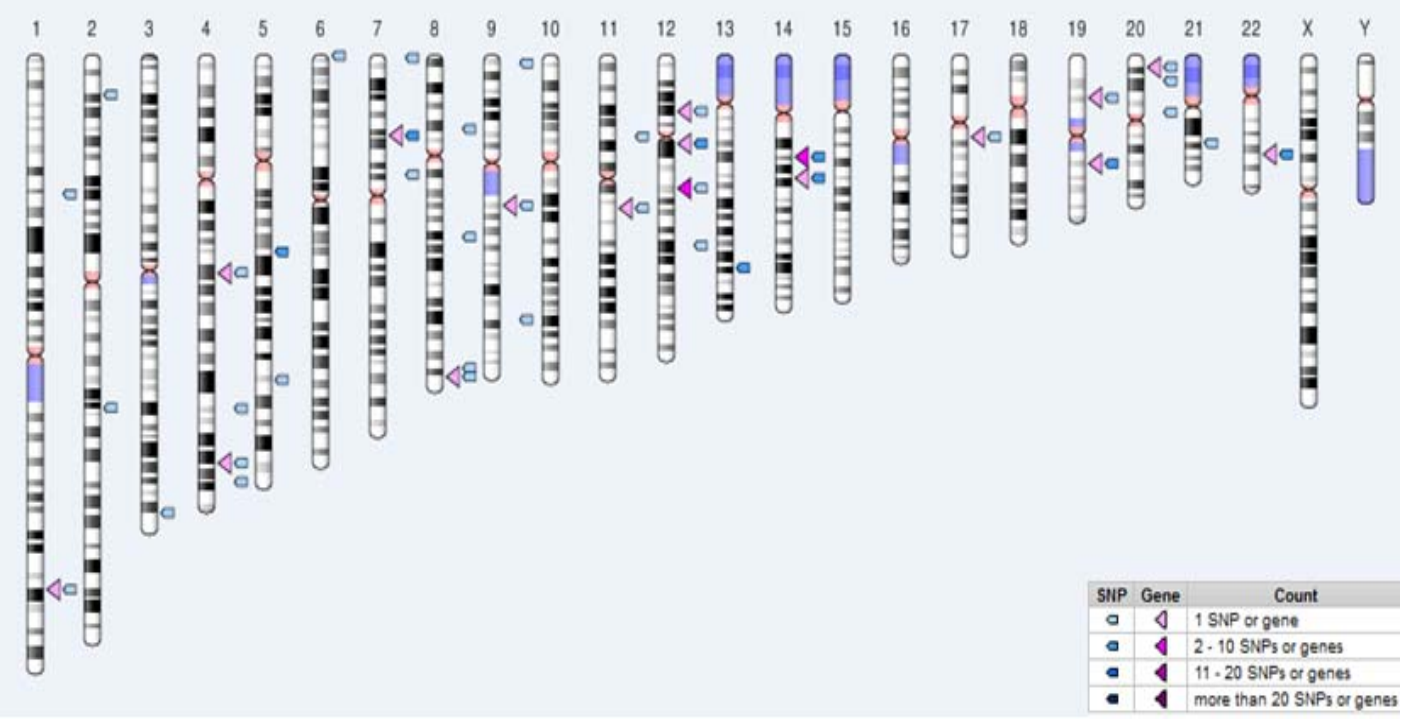

Figure 18. Genome view of single nucleotide polymorphisms or associated genes in age-specific associations based on $P$ values

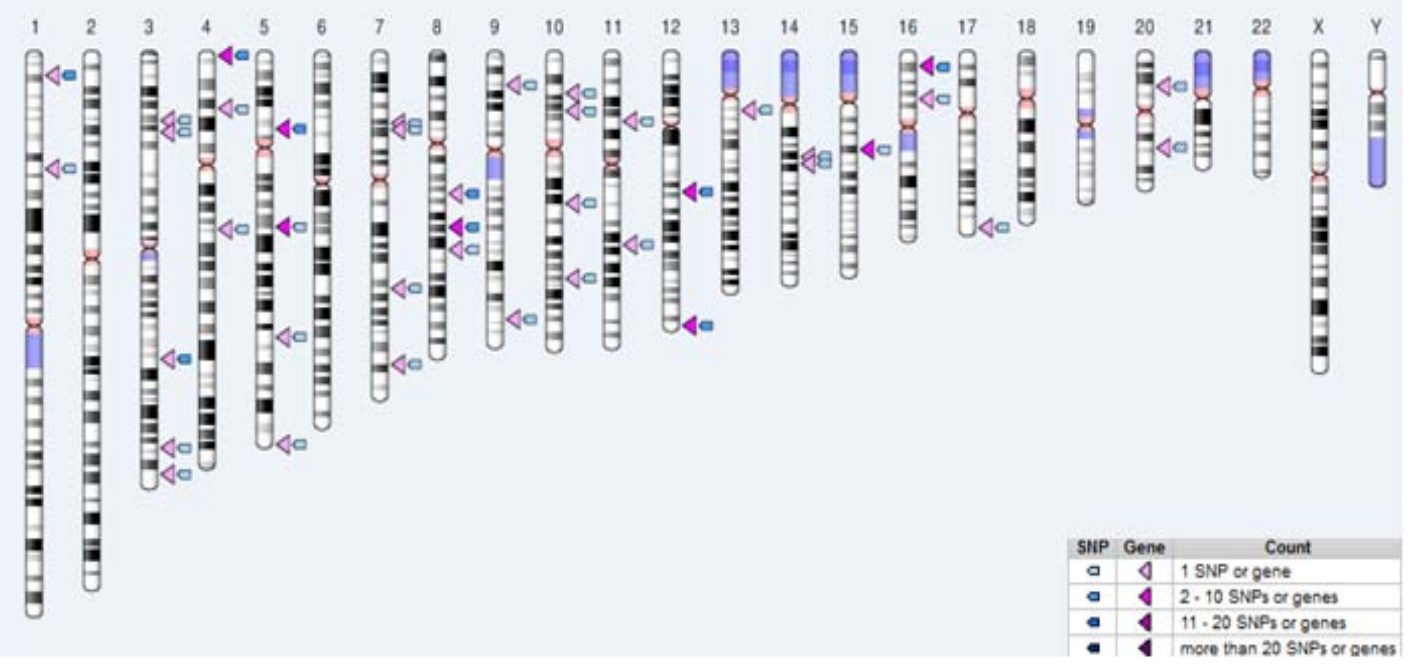

Figure 19. Genome view of single nucleotide polymorphisms or associated genes in age-specific associations based on effect size 
Table 20. SNPs showed age-specific associations in childhood ALL risk with the highest effect sizes $($ OR $>9$; $P$ value $<$ 0.05)

\begin{tabular}{|c|c|c|c|c|c|c|c|c|c|}
\hline \multirow[b]{2}{*}{ SNP } & \multirow[b]{2}{*}{ Gene/region } & \multirow[b]{2}{*}{$\operatorname{Chr}^{\dagger}$} & \multirow[b]{2}{*}{$\begin{array}{c}\text { Minor } \\
\text { allele }\end{array}$} & \multirow[b]{2}{*}{ Feature } & \multicolumn{2}{|c|}{$\begin{array}{l}\text { Minor allele } \\
\text { frequency }\end{array}$} & \multirow[b]{2}{*}{ ORallele $(95 \% \text { CI })^{*}$} & \multirow[b]{2}{*}{ P value** } & \multirow[b]{2}{*}{$\begin{array}{c}\text { P value } \\
(\text { permutation })^{\dagger \dagger}\end{array}$} \\
\hline & & & & & $1-5 y$ & $6-10 y$ & & & \\
\hline rs17109582 & DIO1 & 1 & $\mathrm{C}$ & intron & 0.09 & 0.007 & $12.46(1.67-93.01)$ & 0.01 & 0.006 \\
\hline rs7304215 & TMEM132D & 12 & $\mathrm{G}$ & intron & 0.07 & 0.007 & $11.25(1.47-85.90)$ & 0.02 & 0.01 \\
\hline rs10999147 & AIFM2 & 10 & $G$ & coding & 0.07 & 0.007 & $10.89(1.43-83.02)$ & 0.02 & 0.01 \\
\hline rs7330286 & $13 q 33.2$ & 13 & $\mathrm{~T}$ & intergenic & 0.07 & 0.007 & $10.01(1.33-75.63)$ & 0.03 & 0.01 \\
\hline rs41309927 & SEC23B & 20 & $\mathrm{~A}$ & coding & 0.07 & 0.007 & $9.70(1.28-73.39)$ & 0.03 & 0.02 \\
\hline rs36086854 & CFAP99 & 4 & $\mathrm{~A}$ & coding & 0.06 & 0.007 & $9.42(1.23-72.26)$ & 0.03 & 0.02 \\
\hline rs17409162 & ANO10 & 3 & $\mathrm{~A}$ & coding & 0.06 & 0.007 & $9.24(1.20-71.06)$ & 0.03 & 0.02 \\
\hline rs13129052 & $4 \mathrm{p} 16.3$ & 4 & $\mathrm{G}$ & intron & 0.06 & 0.007 & $9.24(1.20-71.06)$ & 0.03 & 0.02 \\
\hline
\end{tabular}

$* \mathrm{OR}_{\text {interaction }}$ per allele $\left(\mathrm{OR}_{\text {allele }}\right)$ for the additive model; $* * P$ value adjusted for genomic control; ${ }^{\dagger} \mathrm{Chr}=\mathrm{Chromosome}, \mathrm{SNP}=$ single nucleotide polymorphism; ${ }^{\dagger} \mathrm{P}$ value permutation = point wise $\mathrm{P}$ value from maxT permutation analysis after 10,000 permutation 
The Manhattan plot (Figure 20) was generated to visualize the data. Like genderspecific associations, this study yielded several risk markers which may increase the risk of ALL in a specific age group.

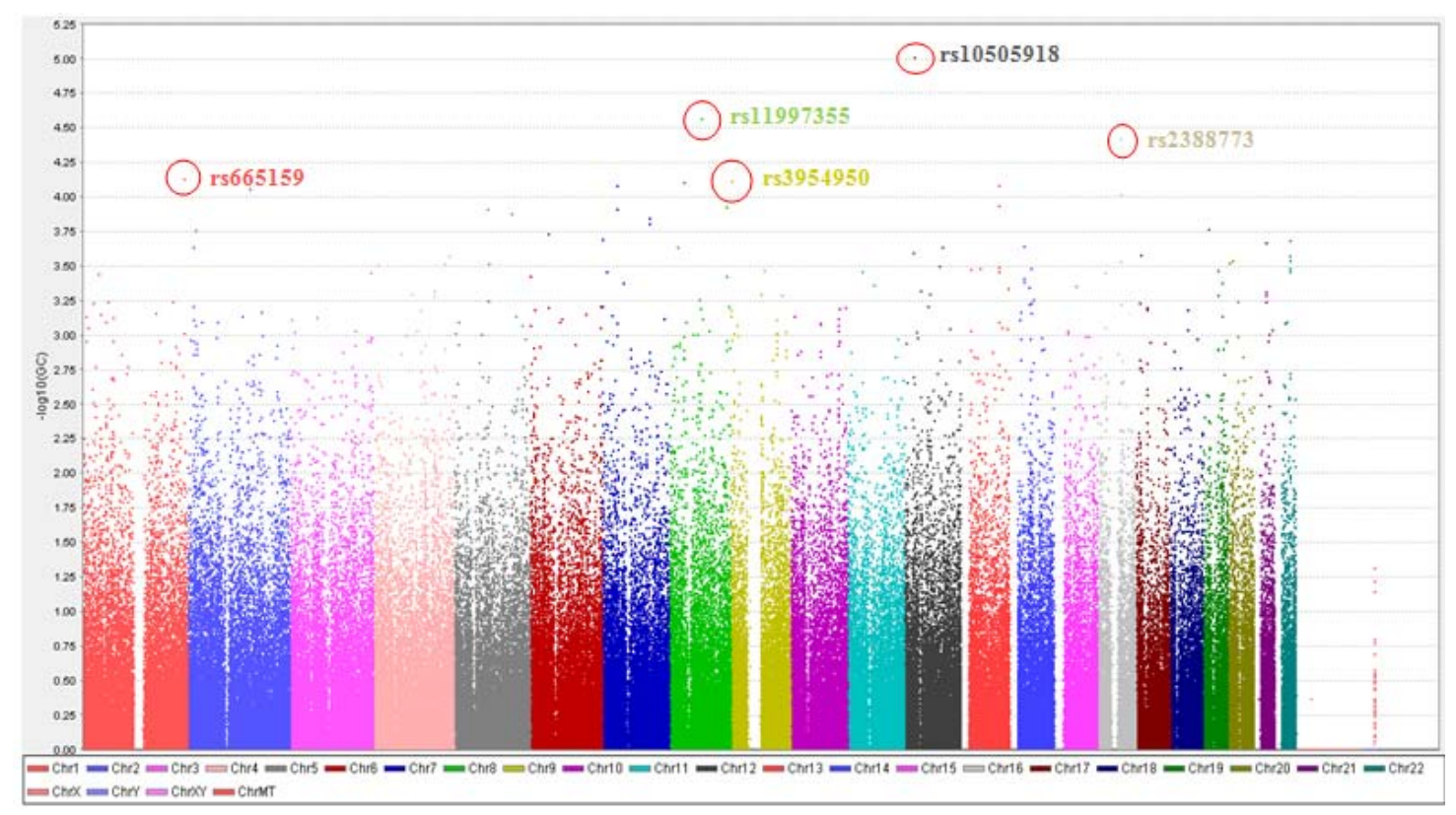

Figure 20. Manhattan plot for age-specific association analysis for childhood acute lymphoblastic leukemia risk

The $\mathrm{x}$-axis represents position on each chromosome and the $\mathrm{y}$-axis shows the $\mathrm{P}$ value on logarithm scale from the additive genetic model

Like gender-specific associations, results based on the effect size again showed associations with more missense (resulting in amino acid change) variations than results based on the P value (24 vs 6), including correlated variants (Appendix Table 16 and Table 17). One SNP, rs75411676, results based on effect size, also showed a nonsense variation. Nonsense variation is the most severe change, as it prematurely stops protein formation. RegulomeDB and F-SNP (explained in Chapter 4) were assessed the functionality of all age-specific markers. All six missense variations, results based on P value, showed benign/tolerable effect in resulting protein formation. Four SNPs 
(rs2101919, rs237318, rs6883 and rs6972158) were located in transcription factor binding sites, and may affect gene regulation, as they have more chance of regulatory effect (Appendix Table 16 and Table 17). A total of 24 SNPs (RegulomeDB $\leq 3 \mathrm{a}$ and F$\mathrm{SNP} \geq 0.2$ ), results based on the $\mathrm{P}$ value, yielded much higher functional score by at least one algorithm. Two SNPs (rs139886 and rs9897794), scored "1f" (RegulomeDB), were suggested most credible functional variants. Nine SNPs scored a much higher functional score $(\geq 0.5)$ for F-SNP. Results suggest these associated SNPs may be the main causal SNPs rather than proxy associations. Correlated SNPs with age-specific association markers, when complemented with RegulomeDB, yielded 34 highly functional variants. One SNP (rs4821717) scored "1b". Moreover, one SNP (rs9897794) scored "1d", and another seven SNPs scored "1f”. Several age-specific risk markers also yielded multiple functional variants, including missense variations. These correlated functional variants might be the main causal SNPs for which these age-specific risk markers may be proxies. Eleven SNPs (results based on the P values) had potential eQTL effects (explained in chapter4) on thirteen genes (Appendix Table 18). Six highly functional correlated SNPs (results based on the P values) showed eQTL effects on 3 unique genes.

Ten SNPs were located in TFBSs (Appendix Table 16 and Table 17), and may influence disease risk by change in binding site. Results based on effect size showed 28 SNPs with higher functional score by at least one algorithm. It again suggests that these SNPs might be causal variants rather than indirect associations. The SNP rs3204798 showed a much higher functional score (0.91) by F-SNP. Functional analysis of correlated SNPs also showed 36 unique risk variants with much higher functional scores or missense variations. One SNP (rs12632793) scored “1d" and two SNPs (rs16909527 
and rs373690) scored "1f". The eQTL analysis yielded 3 SNPs with potential effect on 14 genes (Appendix Table 18). Additional bioinformatic analysis of correlated SNPs suggested that several of these associated risk markers might be proxy for highly functional correlated SNPs. Results based on effect size were enriched with more missense variations with deleterious effect, and also showed more functional variants. Overall, our bioinformatic analyses yielded highly functional variants with potential regulation effect.

As for the gender-specific associations, the SNPnexus provided valuable information from the GWAS catalogue and the COSMIC database. A total of six SNPs were previously associated with some types of traits or diseases (Appendix Table 9), including chronic lymphocytic leukemia (CLL) (rs7169431) and nasopharyngeal carcinoma (rs28421666). The COSMIC database yielded three SNPs, rs34099167, rs762562 and rs1800141, which may increase the risk of stomach cancer, pancreas cancer and large intestinal cancer, respectively.

Like gender-specific associations, the Comparative Toxicogenomics Database (CTD) was also used to examine associated genes for their interaction with environmental pollutants (Appendix: Table $19 \& 20$ ). A detailed list with appropriate references can be found in the appendix. Most of the identified genes or nearest genes physically located at two sides of SNPs, in case of intergenic variants, have shown potential effect of several chemicals, such as poly aromatic hydrocarbons (benzo(a)pyrene), tobacco smoke (benzo(a)pyrene, benzo(a)pyrene diol epoxide, nicotine), pesticides (insecticides, fungicides, herbicides), vehicle emissions, occupational exposures (formaldehyde), polychlorinated biphenyls, bisphenol A, and 
hormones (estrogen, progesterone and testosterone). However, several of these chemicals have previously showed association with childhood ALL, but evidence is limited.

We also predicted the deleterious effect of missense variations, including statistically correlated SNPs, associated with gender- and age-specificity using SNPnexus database (Appendix Table 21 and Table 22). A total of eighteen SNPs from genderspecific associations and seven SNPs from age-specific associations yielded deleterious effects. All of these deleterious missense variations were associated with results based on the highest effect sizes.

\subsubsection{Time-to-event analysis}

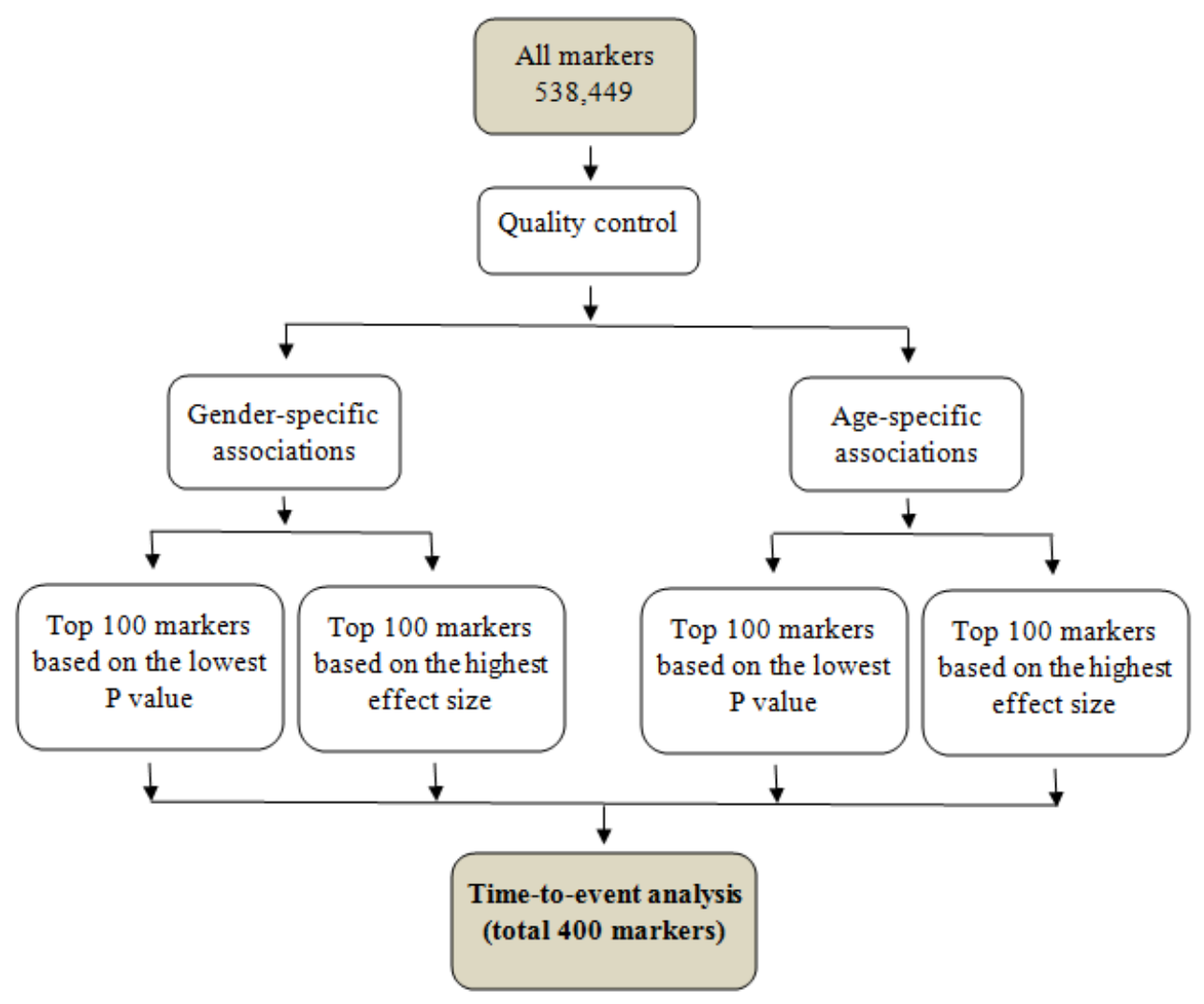

Figure 21. A flow chart explaining marker selection for time-to-event analysis 
Table 21. Time-to-event analysis of identified gender- and age-specific childhood ALL risk markers at the significance level $<1 \times 10^{-4}$

\begin{tabular}{|c|c|c|c|c|c|c|c|}
\hline & & Overall & & Male & & Female & \\
\hline SNP & $\begin{array}{c}\text { Log rank } \\
\text { P value }\end{array}$ & HR (95\% CI) & $P$ value & HR (95\% CI) & $P$ value & HR (95\% CI) & $P$ value \\
\hline rs10505918 $8^{(\mathrm{D})^{*}}$ & $<1 \times 10^{-4}$ & $1.69(1.27-2.24)$ & $<1 \times 10^{-3}$ & $1.55(1.06-2.25)$ & 0.02 & $1.93(1.25-2.96)$ & $3 \times 10^{-3}$ \\
\hline rs2949827 $7^{(\mathrm{D})^{*}}$ & $<1 \times 10^{-4}$ & $0.54(0.38-0.77)$ & $1 \times 10^{-3}$ & $0.72(0.46-1.12)$ & 0.15 & $0.39(0.23-0.66)$ & $<1 \times 10^{-3}$ \\
\hline $\mathrm{rs} 2212824^{(\mathrm{D})^{*}}$ & $<1 \times 10^{-4}$ & $1.76(1.30-2.38)$ & $<1 \times 10^{-3}$ & $1.76(1.20-2.59)$ & $4 \times 10^{-3}$ & $1.76(1.09-2.83)$ & 0.02 \\
\hline rs1209145 ${ }^{(\mathrm{D})^{*}}$ & $<1 \times 10^{-4}$ & $1.80(1.31-2.48)$ & $<1 \times 10^{-3}$ & $1.62(1.05-2.51)$ & 0.03 & $2.05(1.28-3.27)$ & $3 \times 10^{-3}$ \\
\hline rs1209145 ${ }^{(\mathrm{D})^{*}}$ & $<1 \times 10^{-4}$ & $1.76(1.28-2.41)$ & $<1 \times 10^{-3}$ & $1.62(1.05-2.51)$ & 0.03 & $2.05(1.28-3.27)$ & $3 \times 10^{-3}$ \\
\hline rs $738027^{(\mathrm{D})^{*}}$ & $<1 \times 10^{-4}$ & $1.62(1.22-2.15)$ & $1 \times 10^{-3}$ & $1.62(1.10-2.38)$ & 0.01 & $1.62(1.06-2.47)$ & 0.03 \\
\hline rs2101919 ${ }^{(\mathrm{D})^{*}}$ & $<1 \times 10^{-4}$ & $0.61(0.46-0.80)$ & $1 \times 10^{-3}$ & $0.64(0.44-0.94)$ & 0.02 & $0.56(0.37-0.86)$ & $8 \times 10^{-3}$ \\
\hline rs9809647 $7^{(\mathrm{D})^{*}}$ & $<1 \times 10^{-4}$ & $0.60(0.45-0.78)$ & $1 \times 10^{-3}$ & $0.57(0.39-0.84)$ & $4 \times 10^{-3}$ & $0.64(0.42-0.98)$ & 0.04 \\
\hline rs $10133677^{(\mathrm{D})^{*}}$ & $<1 \times 10^{-4}$ & $1.78(1.28-2.46)$ & $1 \times 10^{-3}$ & $1.65(1.05-2.61)$ & 0.03 & $1.92(1.21-3.06)$ & $6 \times 10^{-3}$ \\
\hline rs $10999147^{(\mathrm{D})^{* *}}$ & $<1 \times 10^{-4}$ & $2.55(1.59-4.08)$ & $<1 \times 10^{-3}$ & $2.01(1.09-3.71)$ & 0.03 & $3.90(1.85-8.22)$ & $<1 \times 10^{-3}$ \\
\hline
\end{tabular}

(D): Dominant genetic model; ${ }^{*}$ Age $\mathrm{P} ;{ }^{* *}$ Age OR; $\mathrm{HR}=$ hazard ratio; $\mathrm{CI}=$ confidence interval 
Time-to-event analysis was performed on the top one hundred SNPs for genderand age-specific associations (Figure 21). The log rank test was used for different genetic models (additive, dominant and recessive), to compare the differences between time to having leukemia between two (dominant or recessive genetic model) or three genotypes (additive genetic model). SNPs, which showed statistically significant associations $(\mathrm{P} \leq$ 0.05) with age-at-onset, are listed in Appendix Table 23. KM plots, only for the SNPs with the lowest P values (by log rank), are illustrated in the result section. The Cox proportion hazard modeling was also performed for all statistically significant associations $(\log$ rank $\mathrm{P}$ value $\leq 0.05)$ with age-at-onset to calculate effect size (hazard ratio).

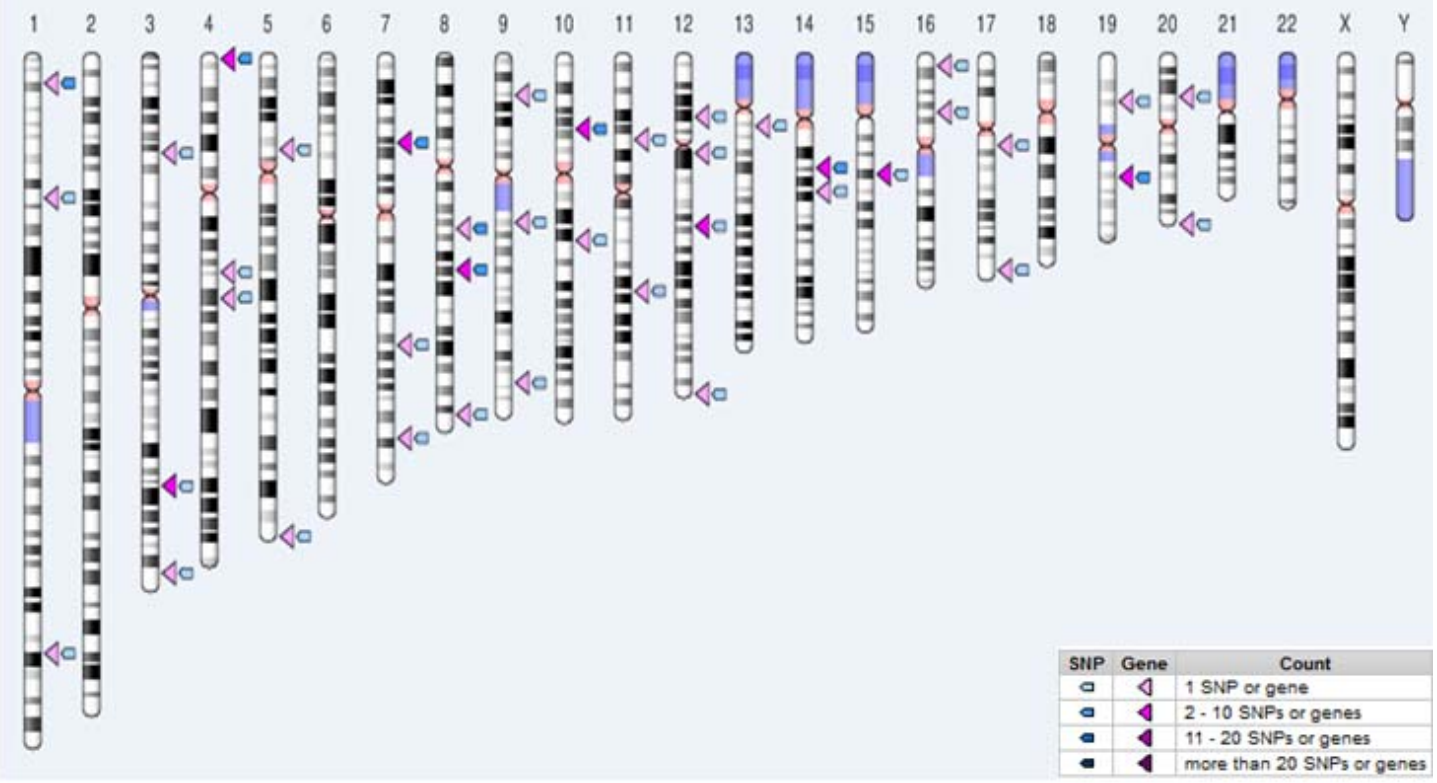

Figure 22. Genome view of single nucleotide polymorphisms or associated genes with age-at-onset 
A total of 196 SNPs, based on the log rank test, yielded statistically significant associations $(\mathrm{P} \leq 0.05)$ with age-at-onset (Table 21 and Figure 22$)$. The maximum number of associations $(\mathrm{n}=13)$ with age-at-onset were observed on chromosomes 1,8 , 12 and 14. Among the top one hundred markers based on effect size for age-specific association results, 91 markers showed associations with age-at-onset. Gender-specific association (effect size results) yielded the lowest number of associations $(\mathrm{n}=11)$ with age-at-onset. Most of these associations best fitted in the dominant genetic model. Few of these SNPs were also correlated. A total of ten SNPs yielded P values $<1 \times 10^{-4}$ with age-at-onset (Table 23).

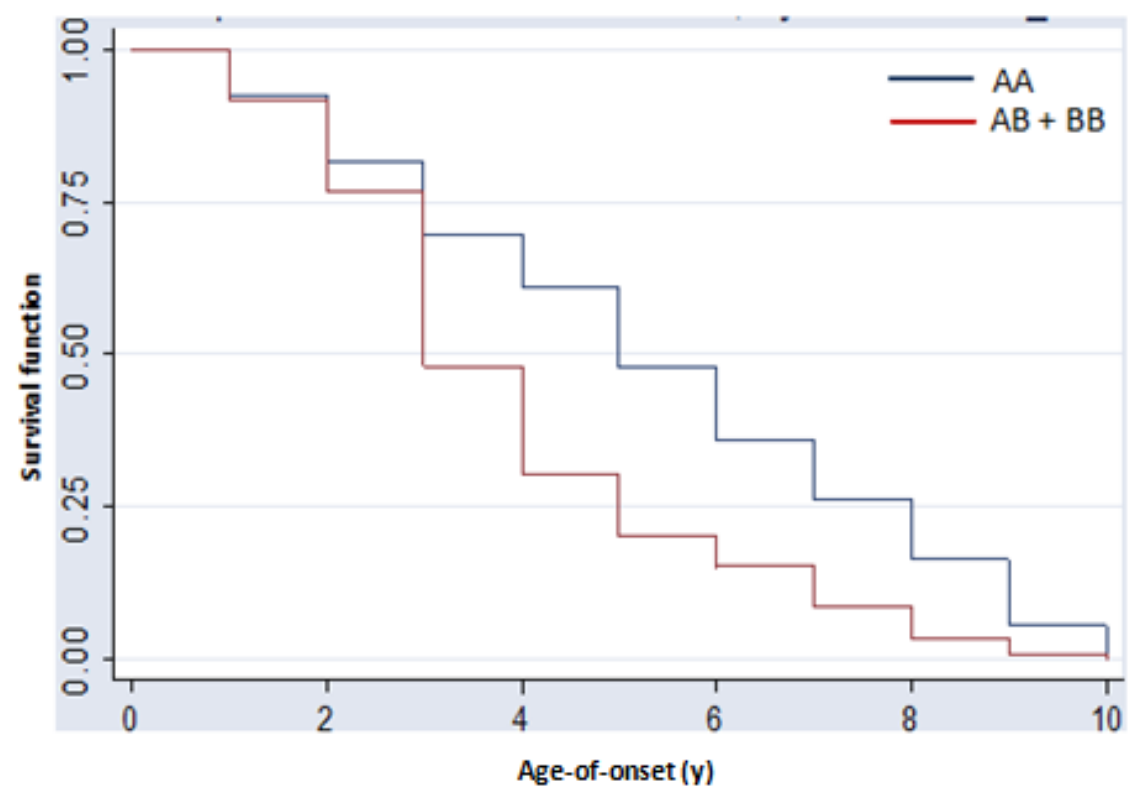

Figure 23. Kaplan-Meier plot estimates for age-at-diagnosis in childhood ALL with rs10505918 genotypes

$\mathrm{AA}=$ wild-type $\mathrm{AB}=$ heterozygote $\mathrm{BB}=$ homozygote variant. Note: $\mathrm{X}$-axis represents age-of-onset in years, and Y-axis represents survival function. Plot compares genotypes and age-of-onset. Dominant genetic model.

The SOX5 rs10505918, which was the top hit for age-specific results (Table 23), also yielded the most statistically significant association with age-at-onset (Figure 23). Childhood ALL cases who possessed heterozygote (AB) and homozygote variants (BB) 
developed ALL on average 2 year earlier than participants who were AA genotype (wildtype) for rs 10505918 (median age, 3 years vs 5 years). The HR was 1.69 that suggests 1.69 times increased risk of early-onset ALL for children possessing heterozygote (AB) and homozygote (BB) genotype.

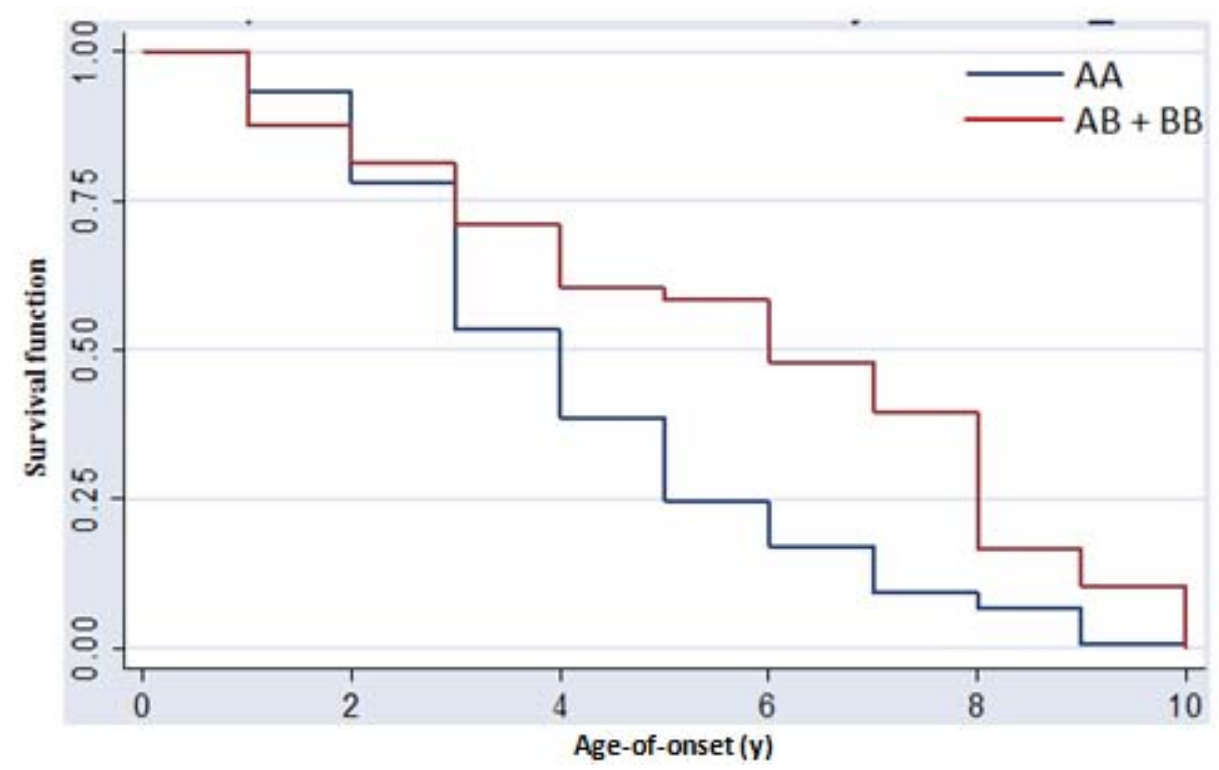

Figure 24. Kaplan-Meier plot estimates for age-at-diagnosis in childhood ALL with rs2949827 genotypes

$\mathrm{AA}=$ wild-type $\mathrm{AB}=$ heterozygote $\mathrm{BB}=$ homozygote variant. Note: $\mathrm{X}$-axis represents age-of-onset in years, and $\mathrm{Y}$-axis represents survival function. Plot compares genotypes and age-of-onset. Dominant genetic model

Likewise, SNP rs2949827 also showed a low P value $\left(<1 \times 10^{-4}\right)$, however, HR was in opposite direction $(\mathrm{HR}=0.54)$ (Figure 24$)$. The HR 0.54 suggests nearly two times increased risk for early-onset of childhood ALL for children possessing wild-type genotype (AA). Children homozygote for wild-type (AA) developed ALL on average 2 years earlier than children who were heterozygote $(\mathrm{AB})$ and variant genotype $(\mathrm{BB})$ (median age 4 years vs 6 years). Several of these associations were only statistically significant $(\mathrm{P} \leq 0.05)$ in one gender. Two associations (rs17170286 and rs 1800141) with 
age-at-onset were gender-specific $\left(\mathrm{P}_{\text {interaction }} \leq 0.05\right)$. Both associations were in the same direction but differed in magnitude. For example, overall HR for rs17170286 was 1.60 (P $=0.03$ ), however, when results were stratified by gender, males showed a much higher increased risk $2.36(\mathrm{P}=0.003)$. HR for female was $1.12(\mathrm{P}=0.72)$.

\subsubsection{Gene enrichment analysis}

Gene enrichment analysis was performed to examine the pathways associated with male or the age group 1-5 year. The main purpose was to assign biological meanings to some group of genes. Our results did not yield any statistically significant association with any pathways.

\subsubsection{Previously identified gender- and age-specific associations}

We also analyzed previously identified gender-specific associations in childhood ALL. To avoid any discrepancy from previous associations, we performed same genetic models for association analysis. Our results did not replicate all previous gender-specific associations (Appendix Table 24). Two SNPs, rs419788 and rs224589, showed the lowest P value, 0.06 and 0.08 , respectively. Like gender-specific associations, previously identified age-specific childhood ALL SNPs did not show the same association in the present study (not reported). Most of the previous associations were either with age-atonset or different age groups.

Previous studies did not counteract the problem of multiple comparisons. Even, few studies used adults and convenient samples as a control group. Environmental factors play a major role in disease association, and different geographical regions (USA vs UK) can also increase the chance of replication failure, as few of these studies were done at 
the United Kingdom. Many non-cancer gender-specific associations were also analyzed, and two of the previously identified associations, rs3131622 (type 1 diabetes) and rs 10195252 (waist-height ratio) were statistically significant $(\mathrm{P}$ value $<0.05)$. 


\section{DISCUSSION}

To our knowledge, this is the first GWAS, which used a gender- and age-specific approach to unravel childhood ALL risk variants. We reported the statistically most significant top one hundred results. Permutation testing was performed to eliminate chance findings (explained in Chapter 4). These results are free from multiple comparison issues, as each permutation on each SNP assesses the role of chance. The rs4813720 (RASSF2), rs231237 (HSPB6) and rs7323018 (13q14.1) SNPs showed the most statistically significant gender-specific associations. Likewise, the SNP rs10505918 (SOX5) showed the lowest $\mathrm{P}$ value for age-specific associations, and also yielded the most statistically significant association with age-at-onset.

Unlike previous GWASs, as a second approach, we also analyzed results based on the effect size, as effect size helps to explore clinically meaningful results, which may not have reached statistical significance due to insufficient power ${ }^{201,202}$. Again, we applied permutation testing for the highest ranking results (top 100 associations), based on the effect size, to eliminate chance findings. Two HLA-DQA1 missense SNPs (rs12722042 and rs12722039) showed the highest effect sizes for gender-specific associations. Several other SNPs, such as rs11992342 (ADAM28) and rs80040922 (UMODL1) also yielded the highest effect sizes for gender-specific associations. The SNPs rs 17109582 (DIO), rs7304215 (TMEM132D), rs10999147 (AIFM2) and rs7330286 (13q33.2) yielded the highest effect sizes for age-specific associations.

Among 400 gender- or age-specific variants, only14 variants were previously

observed associations with other diseases/traits, including CLL (rs7169431) ${ }^{203}$ and nasopharyngeal carcinoma (rs28421666) ${ }^{204}$. The CLL association (rs7169431) was a risk 
association $\left(\mathrm{OR}=1.36, \mathrm{P}=4.74 \times 10^{-7}\right)$ in a previous GWAS. This SNP is located at chromosome 15 , between the NEDD4 and RFX7 genes. Our results provide further evidence for genetic susceptibility of this SNP in childhood ALL with an increased risk to the age-group 1-5 years. Bioinformatic analysis did not suggest any functional mechanism for increased risk in this age group. Experimental studies are needed to understand the mechanism of this SNP on the age effect.

In this study, we also unmasked several associations located within the HLA region at rs28421666, located between the HLA-DRB1 and HLA-DQA1 loci, rs3130161, located between HLA-DPB1 and COL11A2, and two missense SNPs (rs12722042 and rs 12722039) located in the HLA-DQA1 gene. Among all of these previous associations, the rs28421666 SNP has shown a protective association with nasopharyngeal carcinoma $^{204}$. Another SNP, rs3130161, has yielded an association with type 1 diabetes $(\mathrm{T} 1 \mathrm{D})^{205}$. Both of these results were linked with age-specific associations in childhood ALL risk. These results suggest a role for HLA in childhood ALL risk with age specificity. The HLA region is the most polymorphic region in the human genome, which plays an important role in the regulation of the immune system. Although the role of HLA in ALL etiology is not known, several previous studies suggest the role of HLA in childhood ALL risk with gender differential ${ }^{123-125,206-208}$. Most of the previously identified HLA associations in childhood ALL are in the HLA class II region. All HLA associated SNPs are also located in the same region. All of these associations yielded significant associations only for males (rs12722042 and rs12722039) or the age group 1-5 years (rs28421666 and rs3130161), when we ranked results based on the highest effect sizes for gender- and age-specific associations. The rs3130161 SNP is statistically 
correlated with a much higher functional SNP, rs3129203. It suggests the statistically correlated SNP might be the main causal SNP, which can affect the gene regulation through transcription factor binding site. Extensive bioinformatic screening of these SNPs, excluding rs3129203, did not implicate a mechanism behind gender- and agespecificity in childhood ALL risk, except for rs3130161. Further functional studies are warranted to understand the mechanism of these SNPs in sub groups (male gender and age group 1-5 year) of children with ALL.

Among previously identified associations, several of these variants were associated with autoimmune disorders, such as T1D (rs3130161 and rs4948088) 205,209 MS (rs2116078) ${ }^{210}$, psoriatic arthritis (rs12188300) $)^{211}$ and Crohn disease (CD) $(\text { rs798292) })^{212}$. The MS association (rs2116078) was with age-at-onset (mean age $=34$ $y)^{210}$. Our results also yielded an age-specific association (rs2116078) in childhood ALL risk with an increased risk to age-group 1-5 years. Previous epidemiological studies have shown an increased risk of hematological malignancies with autoimmune disorders ${ }^{213-218}$. This observation has been commonly noted in MS and risk of leukemia/lymphoma in adults $^{213}$. Epidemiological studies have also shown four times increased risk of ALL in children of women with $\mathrm{MS}^{214}$. Thus, the MS risk variant rs2116078 might be commonly shared between MS and childhood ALL in age-specific manner, as previously also observed $^{130}$.

The MAGI2 (membrane associated guanylate kinase, WW and PDZ domain containing 2) rs798292 has been previously associated $(\mathrm{OR}=1.30, \mathrm{P}=1.01-1.69)$ with CD in the Caucasian population ${ }^{212}$. A previous GWAS on childhood ALL relapse also suggests the role of the MAGI2 (rs1496766) gene in childhood ALL risk ${ }^{219}$. The two 
polymorphisms (rs798292 and rs1496766) were also not statistically correlated, which suggests independent associations. Bioinformatic analyses of gene expression profiles of B-cell precursor childhood ALL also suggests that the MAGI2 gene may increase the risk of childhood ALL ${ }^{220}$. Alternatively, MAGI2 inhibits cell migration and proliferation through PTEN (phosphatase and tensin homolog), a tumour suppressor gene. PTEN is a key regulator in apoptosis, and defects in apoptosis can increase leukemia risk ${ }^{221,222}$. Moreover, a study on adult B-ALL patients has shown decreased PTEN activity ${ }^{223}$. Empirical analysis also suggested regulatory effect of rs798292 on three genes (MYST2, $A T A D 2 B$ and $E I F 1 A X)$. It means that the SNP, rs798292, is a putative regulation site and it has allele-specific effects on gene expression. Thus, rs798292 may play a significant role in childhood ALL risk through regulatory effects or through PTEN interaction.

A cohort of 24,052 patients of T1D in Sweden showed a co-morbidity between early-onset leukemia and T1D (standardized incidence ratio $=8.30 ; \mathrm{P}=4.91-13.14)^{218}$. The two polymorphisms (rs3130161 and rs4948088) previously identified as risk variants for T1D $\mathrm{D}^{205,209}$ also yielded age-specific associations in childhood ALL. The SNP, rs3130161, showed an association with the age-group 1-5 years and the rs4948088 SNP yielded an association with the age-group 6-10 years. Autoimmune diseases are the most common group that have shown associations with our results presumably due to them being more common in one sex. This epidemiological evidence suggests biological plausibility between few autoimmune disorders and hematological malignancies, including childhood ALL with gender-specificity ${ }^{213-218}$. Interestingly, autoimmune disorders are the most common group that have shown associations with our results due to them being more common in one gender ${ }^{110}$. 
Childhood ALL shows a consistent association with recurrent miscarriages, specifically in males ${ }^{224}$. The $P R L R$ (prolactin receptor) polymorphism rs 37389 is involved in recurrent miscarriages ${ }^{225}$. The role of the $P R L R$ gene is not established in leukemogenesis, but a higher expression of this gene in cancer etiology cannot be neglected $^{226}$. Several other previous associations are with HBA1c level (rs552976) and fasting glucose level (rs563694). One SNP, rs560887, yielded multiple associations with glycemic traits.

In this study, we have also observed a statistically significant difference for the frequency of the ERCC1 (excision repair cross-complementation group 1) rs762562 polymorphism between two age groups. The functional role of ERCC1 is not clear in leukemia. Similar results, however, with different polymorphisms (rs3212986 and rs11615), have previously shown associations with childhood ALL risk in a Chinese population $^{227}$. Moreover, the rs3212986 SNP has yielded a much higher risk $(\mathrm{OR}=1.87$; $\mathrm{P}=0.04)$ in the age group $<8$ years compare to age group $>8$ years $(\mathrm{OR}=1.44 ; \mathrm{P}=$ 0.24). Both SNPs (rs3212986 and rs11615), in the previous study, have shown an increased risk in males $(\mathrm{OR}=1.94 ; \mathrm{P}=0.02$ and $\mathrm{OR}=2.36 ; \mathrm{P}=0.04$, respectively $)$ in comparison to females $(\mathrm{OR}=1.19 ; \mathrm{P}=0.57$ and $\mathrm{OR}=0.46 ; \mathrm{P}=0.05)$. Furthermore, rs 11615 also yielded a gender-specific association in the present study $(\mathrm{P}=0.03)$, with an increased risk in males. Thus, results suggest that ERCC1 gene may be a potential target to examine gender- and age-specificity in childhood ALL risk. The SNP rs762562 is located in an overlapping region of ERCC1 and CD3EAP. The rs762562 SNP shows a missense variation in the CD3EAP gene, which suggests an obvious change in protein 
function. The SNP rs762562 also showed a somatic mutation by bioinformatic analysis (explained later). Thus, it increases the validity of our results.

The SNP, rs9282777, is present in the coding region of the LMO2 (LIM only protein 2) gene and yielded a much higher risk in males. The LMO2 gene plays a crucial role in hematopoietic stem cell development, angiogenesis and embryogenesis ${ }^{228,229}$. Higher expression of the $\mathrm{LMO} 2$ gene during immature stages of lymphocyte formation has been observed ${ }^{230}$. The $L M O 2$ gene is considered as a cancer causing gene, and it increases the risk of T-cell ALL ${ }^{228,231-233}$. Studies also report the higher expression of the LMO2 gene in B-cell ALL and other lymphomas ${ }^{229,234}$. Our result suggests that rs928277 may play a significant role in B-cell etiology. Bioinformatic screening did not suggest any mechanism in childhood ALL risk with male susceptibility. More functional study is needed to understand the mechanism of rs9282777 in gender differential in leukemia risk.

The rs 10505918 SNP showed the most statistically significant age-specific association. This SNP is located in the SOX5 (SRY (sex determining region Y)-box 5) gene, which is involved in the regulation of embryonic development, however, its role in childhood ALL etiology is not clear. Studies suggest that the SOX5 gene expresses in Bcells and has a functional role in late B-cell development and B-cell differentiation ${ }^{235}$. This gene is also highly expressed in B-cell lymphomas ${ }^{236}$. The SNP rs10505918 also yielded the most statistically significant association with age-at-onset $\left(\mathrm{P}<10^{-4}\right)$. Interestingly, the SOX5 gene, however, a different polymorphism (rs7294845), also showed a gender-specific association based on the $\mathrm{P}$ value in this study. This evidence altogether suggests a role of SOX5 in childhood ALL risk with gender- and agespecificity. 
The SNP rs 11992342 yielded a higher effect size for gender-specific associations. This SNP is located in the ADAM28 gene. Recent evidence suggests that ADAM28 is expressed on the surface of human lymphocytes ${ }^{237}$. The role of ADAM28 in leukemogenesis is not established, but our results suggest that ADAM28 polymorphism (rs11992342) was associated with the gender differential in childhood ALL risk with the highest effect sizes. A total of three ADAM28 SNPs (rs11992342, rs7829965 and rs36041430) showed gender-specific associations, however, all three SNPs were statistically correlated. The rs 7829965 is a missense variation, and also yielded a higher score for gene expression regulation, which suggests regulatory effects on binding site.

The ACACA (acetyl-CoA carboxylase alpha) gene is located at chromosome 7, which yielded multiple gender-specific associations (rs12948120, rs8081866, rs2542660, rs2542653, rs725038) with an increased risk in females. ACACA plays an important role in fatty acid synthesis and is regulated by the thyroid hormone ${ }^{238}$. BRCA1 affects fatty acid synthesis through its interaction with $A C A C A$, and this interaction may exert a tumor suppressor function $^{239-241}$. ACACA has also shown associations with breast cancer risk ${ }^{242}$. Childhood leukemia patients have been found to carry $M L L$ fusion to $A C A C A^{243}$. A statistically correlated SNP, rs9330250, yielded a higher functional score, which suggests gene regulatory function the SNP rs9330250. The SNP rs9330250 was annotated as a credible functional variant and may play a mechanistic role in pathogenesis of childhood ALL. Thus, ACACA seems to have an additional biological feature, and it may increase the risk of leukemogenesis ${ }^{244}$.

The N4BP2 (NEDD4 binding protein 2) gene yielded two missense variations (rs61748746 and rs61748749). The encoded protein by this gene binds to B-cell 
CLL/lymphoma 3 (BCL-3) protein ${ }^{245}$. The BCL3 translocation with IGH is found in some patients with childhood ALL ${ }^{246}$, and subsequent overexpression of BCL3 ${ }^{247}$ in childhood ALL. The gender-specific association we found in this study might be the results of obvious change in protein function and its effect on BCL3 expression. However, more functional studies need to prove this hypothesis, as bioinformatic analysis did not unravel any functional mechanism for male-specific childhood ALL.

A recent study has shown fusion of HIP1 to PDGFRB in chronic myelomonocytic leukemia $^{248}$. Furthermore, HIP1 is localized in $7 \mathrm{q} 11.3$ region, and the possibility of $7 \mathrm{q}$ deletions is associated with some acute non-lymphocytic leukemia cases ${ }^{249}$. However, its role in lymphoblastic leukemia is not yet established, but our result (rs6962352) suggests an additional role of HIP1 in the pathogenesis of leukemia. Down syndrome is a known risk factor for childhood ALL. The KIF21A gene shows much higher expression in Down syndrome patients in leukocytes ${ }^{250,251}$. Unfortunately, unavailability of cytogenetic data is a limitation of this study to reach any conclusion. Additional functional studies need to confirm the role of rs11172024 (KIF21A) in childhood ALL etiology.

The rs6151599 SNP yielded an age-specific association result based on the effect size. This SNP is located in a common region shared between the MSH3 (mutS homolog 3) and DHFR (dihydrofolate reductase) gene. Bioinformatic analysis yielded a statistically correlated SNP (rs11749051) with a higher functional score located in the DHFR gene. It suggests that the rs11749051 SNP may be the main causal SNP. Studies suggest the low DHFR expression may be an important prognostic factor in childhood $\mathrm{ALL}^{252,253}$. 
In this functional analysis of gender- and age-specific risk variants, our results yielded nine highly functional SNPs (six for gender-specific associations and three for age-specific associations). Among these SNPs, one SNP (rs6929434) scored 1b and four other SNPs (rs8106959, rs739924, rs139886 and rs9897794) scored 1f. Likewise, rs739924 and rs130069 scored 1 for F-SNP analysis, and rs11751765 and rs3204798 scored 0.9. These SNPs are highly functional and likely to be the causal SNPs. However, in the results we reported all SNPs which showed RegulomeDB score $\leq 3 \mathrm{a}$.

In a genetic association study, functional variants are most likely to be located at nearby genes around associated risk markers ${ }^{254}$. These functional variants are mostly in strong correlation with associated variants and may be the main causal SNPs. Correlation analysis was complemented with functional analysis to identify the causal SNPs. Some gender- and age-specific associated risk variants also yielded several statistically correlated functional variants. A total of 20 statistically correlated risk variants yielded a much higher functional score ( $\leq 1 \mathrm{f})$. rs9913430 (correlated with rs739924) scored 1a, and rs 139852 (correlated with rs4821717) scored 1b. These highly functional correlated SNPs might be the main causal SNPs for which associated results may be proxies. RegulomeDB is a database that explains SNPs with known or predicted functions ${ }^{184}$. Lower scores suggest evidence for a variant to be located in a functional region. It has strong power to separate truly functional variants and provide putative regulation site. A score $\leq 1 \mathrm{f}$ suggests that functional variants are likely to affect the binding site and/or be linked to the expression of a gene target.

Genetic variants play a major role in disease susceptibility by altering gene expressions. The eQTL analysis is a well recognized method in evaluating the effect of 
SNPs in mRNA or protein levels (explained in Chapter 4). eQTL refers explicitly to the genomic locus, which influences the variable mRNA or protein level. We found 15 gender- and age-specific associations with eQTL SNPs. Among all highly functional statistically correlated SNPs, nine SNPs showed significant eQTL expressions in LCLs. These 24 eQTL SNPs yielded 52 eQTL target genes $(n=22$ gender-specific results; $n=$ 30 age-specific results). Multiple eQTL SNPs identified several common eQTL target genes, such as SPATA20, FGD6, LMF1, ZNF420, MRPL14 and SLC13A2, which suggest these genes may contribute to the risk of childhood ALL. The eQTL database was explored to find whether these SNPs are putative regulations sites and can affect gene expressions. For example, the eQTL SNP rs9493135 (gender-specific results based on effect size) located in the 6q23.2 region, regulates several eQTL genes, including PTPN11. This gene is a member of the protein tyrosine phosphatase family and is highly expressed in hematopoietic cells ${ }^{255}$. This gene plays a very important role in Ras and JakStat pathways. Both pathways are significantly associated with leukemogenesis ${ }^{221,255}$.

A total of 21 SNPs (seven gender-specific results and 14 age-specific results) were located in transcription factor binding sites. These binding sites are mostly concentrated within the promoter regions of genes. A promoter is a region of a gene that initiates gene function. These 21 SNPs may therefore have greater functional effects on gene expression. Genetic variants can also alter protein structure and modify disease risk. Fifty-five of gender- and age-specific associations were with missense (31 genderspecific results and 24 age-specific results) SNPs. However, most of these missense SNPs $(n=48)$ were in the top 100 list based on the effect sizes. These missense variations substitute amino acids, which alter the final protein function. Our result also yielded a 
nonsense variation (rs75411676). Nonsense variation is the most severe change, as it prematurely stops the protein formation. These missense or nonsense variations have deleterious effect and can increase the risk of childhood ALL. However, we performed bioinformatic analysis to identify the significance of these variations. PolyPhen and SIFT predicted the damaging effect of 25 missense variations. It suggests that amino acid substitutions will have deleterious effect in protein function. We also explored functional similarity of identified genes for gender- and age-specific associations, by using stringent criteria. We only used eQTL target genes, missense and nonsense variations (only for damaging effect) associated genes, and only if a SNP showed a very high functional score. However, our results did not show any statistically significant pathway associations. Overall, bioinformatic and empirical analyses of gender- and age-specific results, including correlation analysis, yielded several functional variants. These functional variants may be associated with gender and age differential in childhood ALL risk and should be pursued in future studies.

Although gene enrichment analysis did not yield any statistically significant pathway, some genes are associated with several pathways associated with leukemia. As mentioned, most of these gender- and age-specific associated childhood ALL SNPs are novel risk variants, as expected. The role of these SNPs is still not clear in childhood ALL etiology, however, some of these associated genes play a major role in several leukemia associated pathways. For example, Notch1 signaling plays an important role in childhood leukemia ${ }^{256}$. Recent studies have shown the role of MAML1 (mastermindlike1) in the regulation of Notch oncogenic activities in leukemic cells ${ }^{257-259}$. The MAML1 rs41285557 showed a male-specific association in this study. 
The RASSF2 (Ras association (RalGDS/AF-6) domain family member 2) SNP, rs4813720, yielded the lowest $\mathrm{P}$ value for gender-specific associations. RASSF2 is a novel tumor suppressor gene and a member of the Ras family that regulates a wide range of biological process, including Ras signalling and apoptosis ${ }^{259-261}$. Studies suggest that genes affecting the Ras pathway is the most common occurrence in human cancer, including childhood $\mathrm{ALL}^{262,263}$. Likewise, several other genes, RAB28 (rs10003958), ADAMTS12 (rs7700370) and RSU1 (rs4748309) may play a significant role on leukemogenesis through the Ras pathway ${ }^{264}$. The role of gender- and age-specific associated risk variants in the Ras pathway may be important to increase the risk of leukemia.

The HSPB6 (rs231237) gene encodes a heat shock protein and is constitutively expressed in various tissues ${ }^{265}$. A study on hepatocellular carcinoma showed its interaction with P13K, which ultimately results in suppression of the AKT pathway. P13K/AKT pathways also play an important role in childhood leukemogenesis, specifically T-cell ALL ${ }^{221,222}$. Likewise, the CYFIP2 (rs1030270), OSGIN1 (rs62640905), ZNF420 (rs4254428 and rs6510588), AIFM2 (rs10999147) and RASSF3 (rs11175471) genes show TP53 dependent apoptosis and may increase childhood ALL susceptibility ${ }^{266-271}$. Studies suggest that leukemia may be more sensitive to change in TP53 dependent apoptosis ${ }^{126}$. The present findings suggest that the associated variants may alter the pathway mediated by TP53 and increase leukemia risk. Some of these SNPs were missense variations or yielded a much higher functional prediction score.

These pathways may play a significant role in childhood ALL etiology with gender- and age-specificity. However, these results should be interpreted cautiously, as 
none of the age- or gender-specific SNPs or associated genes are directly associated with leukemogenesis. How these SNPs affect on gender- and age-specificity in childhood ALL risk is still not clear. Some studies suggest that genetic variants in the TP53 pathway could interact with gender to affect cancers ${ }^{134,272}$.

Previous GWASs have missed these gender- and age-specific variants as they did not stratify their results by gender and age. Association differing in direction, usually results in an $\mathrm{OR}$ close to the unity $(\mathrm{OR}=1.0)$ with no statistical significance in the overall analysis. These SNPs were dismissed by previous studies for further considerations. Stratified analysis reduces statistical power. However, it is not true in the case of gender- and age-specific associations. In gender- or age-specificity, an overall analysis approach does not have sufficient statistical power to detect the association. If there is a gender- or age-specificity, stratified analysis will yield much higher effect size for a particular group, such as males or the age-group 1-5 years. The statistical power will be greater in this case than combined analysis. Other problems with previous studies were adjusting the results for gender and declaring there is no gender-specific association. Statistical adjustment only rules out confounding by gender, which is not equivalent to gender-specificity ${ }^{26}$. Thus, our approach unraveled novel risk variants, which are associated with a particular gender or age group in childhood ALL risk.

SNPnexus database used COSMIC database to identify SNPs associated with somatic mutations. Somatic mutation is only present in somatic cells. It means that they are not present in all body cells or germ cells. Unlike germline mutations, these mutations are acquired. Somatic mutations either randomly occur or generate by environmental chemicals. Our results yielded several SNPs associated with several cancers through 
somatic mutations. It has been suggested that somatic mutations in cancer cells may be drivers (causing progression of cancer) or passengers (just the results of random mutations). A total of six SNPs, rs61753605 (PRIM2B), rs35693261 (MTG2), rs180041 (LRP1/MIR1228), rs4645656 (ZNF782), rs34099167 (NEK1) and rs762562 (ERCC1/CD3EAP), detected as somatic mutations by COSMIC database. It suggests that these SNPs may cause functional changes that are advantageous for cancer occurrence and/or progression. These somatic mutations were associated with prostate, breast, large intestine, stomach and pancreatic cancers. The LRP1 gene promotes cancer cell invasion by supporting ERK and inhibiting and JNK signaling pathway ${ }^{273}$. ERK signaling pathway is associated with leukemogenesis. Studies suggest that mutation in the NEK1 gene may lead to chromosomal instability, and thus increase the risk of cancer. The ERCC1 gene has previously showed an increase risk of childhood ALL with male- and agespecificity ${ }^{227}$.

In this study, we found a number of associations with gender- and age-specificity. Bioinformatic and empirical analyses suggested that several of associated SNPs may play a functional role in gender and age differential in childhood ALL risk, through change in protein function (missense variations), regulatory effects on gene expression (eQTL) or through alterations of transcription factor binding sites. Although gene enrichment analysis did not yield any pathway to explore gender- and age-specificity, several genomic locations of associated risk variants are associated with leukemogenesis.

In the past few years, GWASs have yielded hundreds of associations for around hundred traits and diseases. However, the effect sizes are modest $(\mathrm{OR}<1.5)^{274,275}$. The median OR for binary traits is 1.28 (interquartile range (IQR) 1.17 to 1.55 ). Most of the 
previous GWASs used genome-wide significance $\left(\mathrm{P}<10^{-8}\right)$ level to show an association. Our results did not reach this significance level for gender- and age-specific associations. In comparison to previous GWASs, our gender- and age-specific analyses, which are based on statistical significance, yielded a much higher median OR (2.44; IQR = 2.22 2.78). Interpretation of an OR, in a case-only analysis, is important, as a statistically significant association with $\mathrm{OR}>1$ only suggests a positive interaction (risk to males or age group 1-5 y). A higher effect size, however, suggests a higher risk to a particular gender or age group. An OR $<1$ in the present study suggests risk to females or to the age group 6-10 years. Even, an inverse relationship has been observed between sample sizes and effect sizes. Studies with larger sample sizes mostly yielded lower effect size associations. Of course, larger sample sizes provide more statistical power to detect even a minute effect size such as $\mathrm{OR}=1.1$, but the clinical or public health utility of these associations is questionable.

Most of the previously identified variants, in general, around $88 \%$, were located in non-coding regions ( $43 \%$ in intergenic region and $45 \%$ in intron). In the present study, around $94 \%$ of associations were in non coding regions (57\% intergenic regions and $38 \%$ in intron). In comparison to $9 \%$ nonsynonymous variations (missense or nonsense) in previous GWASs, our results only showed 3\% of nonsynonymous variations. In case of SNPs location at 3' or 5' untranslated region, results were similar (2\%). Although we used a different GWAS chip, enriched with coding SNPs, gender- and age-specific results, based on SNPs location, differ from the previous GWASs. Results based on the highest effect sizes for gender- and age-specific associations yielded more meaningful SNP locations. Around $67 \%$ of identified associations were located in non-coding region 
of genes (39\% in intergenic region and $28 \%$ in intron). A total of $25 \%$ SNPs were missense variations, and 5\% of SNPs were located in the $3^{\prime}$ or $5^{\prime}$ ' untranslated region. Results based on the effect sizes yielded more meaningful results in comparison to results based on the lowest P values, such as more SNPs in coding region and missense variations.

Genetic variants usually modify disease susceptibility in the presence of environmental exposures ${ }^{276}$. Genetic factors play a predominant role in diseases due to rare and highly penetrant mutations. However, common genetic polymorphisms modify disease susceptibility in the presence of environmental exposure. Unfortunately, in the case of childhood ALL, the only known environmental risk factor is ionizing radiations. In this study, the CTD was used to explore the gender- and age-specific associated genes and their interactions with environmental chemicals ${ }^{277}$. The most common environmental pollutants interacting with the target genes of the SNPs that have shown in the present study were PAH, vehicle emissions, cigarette smoke, pesticides, drugs such as acetaminophen, alcohol and formaldehyde. A detailed list of gene-chemical interactions and appropriate references are provided in the appendix (Table 10, 11, 19 and 20). These environmental chemicals are ubiquitous and show associations with an increased risk for childhood ALL ${ }^{192-196,278}$. Most of these chemicals are classified as class 1 and class 2 carcinogens ${ }^{191}$ or teratogens. Studies suggest that age plays an important role in the susceptibility to environmental toxicants ${ }^{279}$. Because of differential exposure, children are more susceptible to a number of different environmental pollutants, including PAH, air pollution, cigarette smoke and pesticides. Children consume more foods and water per pound of body weight when compared to adults. They breathe air at a faster rate than 
adults. Thus, children are more vulnerable to environmental toxicants. There is also evidence of prenatal exposures and childhood ALL risk, such parental occupational exposure, pesticide exposure, tobacco smoke and air pollutants ${ }^{280}$.

Like age-specificity, studies also suggest gender-specificity in chemical exposures ${ }^{197-200}$. Benzene and ethanol toxicity is much higher in males, regardless of whether exposures occur in utero or adulthood ${ }^{197,}{ }^{198}$. These results suggest the importance of gender-difference in benzene induced toxicity. Likewise, females have an increased risk for acute pesticide-related illness. Exposures during pregnancy may increase the susceptibility of childhood ALL. Thus, age and gender differences in the toxicity of environmental chemicals may play a significant role in ALL biology. However, interaction between environmental pollutants and gender- and age-specific risk markers are not yet known.

Rarity of childhood ALL makes it difficult to study the cause of the disease. Most epidemiological studies of childhood ALL and environmental exposures are case-control studies. Case-control studies mostly rely on parents' recall for environmental exposure. Parents of childhood cancers may recall their memories more thoroughly than healthy controls. Thus, recall bias may inflate the results and introduce systematic error. That is one of the potential reasons for the limited evidence for the involvement of environmental exposures in the development of childhood ALL.

There are very few examples of gender- and age-specific associations in disease risk. A recent genome-wide meta-analysis of asthma reported gender-specific candidate risk markers in IRF1 (rs2549003) and RAP1GAP2 (rs9895098) with regulatory effects in gene regulation as $\mathrm{eQTL}{ }^{281}$. Asthma prevalence also shows intriguing gender-specific 
architecture that differs by age. Males are more susceptible during early childhood and females develop asthma during puberty. That study also confirmed the advantage of gender-specific approach in a genetic association study to understand disease etiology.

Likewise, another study in 5,254 participants (2,833 females and 2,421 males) from Framingham Heart Study has shown 14 eQTL SNPs (13 autosomal and one X chromosome) with gender-genotype interactions and ten eQTL SNPs with age-genotype interactions after multiple comparisons ${ }^{282}$. These eQTL SNPs are associated with several complex traits/diseases. Overall they found 5,866 genes differently expressed between males and females. Interestingly, they have also shown different gender-specific models, such as associations differing in direction and associations differing in magnitude, in gender-genotype interactions, which we also aimed in this study. For example, the C5orf35 rs686798, association with plasma triglyceride levels, has shown opposite effects on the expression of the IL6ST gene, increased expressions in females $(\mathrm{P}=$ 0.0006) and decreased expressions in males $(\mathrm{P}=0.05)$. Another SNP, rs8047080, associated with high-density lipoprotein, has shown higher expression only in males $\left(7.03 \times 10^{-5}\right)$. Thus, these recent studies support the rationale of this dissertation.

These examples are very recent examples of a gender- and age-specific approach, which we had also proposed in this study. Age related eQTL SNPs have also confirmed the effects of genetic variants on gene expression throughout the age span. We examined these associations in our study, however, none of these showed any age- or genderspecific associations in childhood ALL risk. These gender- and age-specific variants might only be associated with particular diseases/traits. 
Most of the previous gender-specific associations in childhood ALL are within the HLA region. Our results further confirm the role of HLA in childhood ALL risk with male-specificity. Early-onset of childhood ALL and HLA associations are new findings and may shed some light on childhood ALL risk. Van Der Meulen et al, recently, have demonstrated the loss-of-function mutation in the X-linked UTX (KDM6A) gene in childhood T-ALL ${ }^{283}$. This UTX mutation was exclusively present in males, which suggest the role of X chromosomes in ALL etiology. Our results did not show any genderspecific or age-specific associations with $\mathrm{X}$ chromosome in the top one hundred associations.

Our results did not replicate the same gender-specific associations for previously identified childhood ALL risk markers, even after applying the same genetic models as used in previous childhood ALL studies. The closest association was the SKIV2L rs419788 $\left(\mathrm{P}_{\text {interaction }}=0.06\right)$. Some of these studies may have suffered from multiple comparison issues. Due to rarity of childhood ALL, case-control study design was used to identify gender-specific associations. Thus, controls should be representative of the general population. One study used adults as a control group ${ }^{125}$, and another study used convenience samples as controls ${ }^{139}$ to identify gender-specific associations. Thus, genderspecific associations might be affected by selection bias, as controls may have different environmental exposures than childhood ALL. Genetic variants modify disease susceptibility in the presence of environmental risk factors, so geographical differences may also apply in some replication failure, as few of these gender-specific associations were studied in the United Kingdom ${ }^{125,128,130 .}$ 
Even after clear heterogeneity in age differences (age peak 1-5 y) in childhood ALL, there is not much effort to unravel genetic risk markers in this age group. More than $50 \%$ of childhood ALL cases belong to the age group 1-5 years. Only four SNPs (rs3212986, rs2279744, rs4132601 and rs10821936) have shown age-specificity in childhood ALL risk $^{51,52,126,227}$, and two of them are known childhood ALL risk markers (rs4132601 and rs10821936) ${ }^{51,52}$. The SNP rs 10821936 was compared between age group 1-10 years and 11-18 years. Another association (rs4132601) was with age-at-onset in childhood ALL risk ${ }^{140}$. rs2279744 and rs10821936 also failed to replicate previous age-specific associations. 


\section{STRENGTHS AND LIMITATIONS}

Due to the rarity of childhood ALL, the sample size of the present study was small. Twenty nine samples were excluded after strict QC steps, further reducing statistical power. Another limitation was external validity of these results, as all of these samples were Non-Hispanic whites. Thus, these results cannot be generalized in any other ethnic population. Our study was also restricted to age group 1-10 years, which also lessened the statistical power. Self-reported gender, age and ethnicity data may be of concern, as misclassification biases. Population-based studies mostly rely on selfreported data, and residual confounding is possible. In terms of gender, we verified discordant gender information by the usage of sex-chromosome genotyping results and excluded the samples in case of ambiguity in gender information. Thus, it resulted as a strength of this study. Self-reported ethnicity may be reliable ${ }^{284}$. To avoid false positive associations due to population stratification, we adjusted our results using genomic controls, which is also a strength of the present study. In the case of age, we depended on self-reported data. Lack of cytogenetic data is another limitation of this study.

Chromosomal aberrations, such as chromosomal trisomies (such as Down syndrome) or chromosomal translocations (fusion of two genes from different chromosomes) are associated with childhood ALL risk. Still these chromosomal aberrations represent only a fraction of cases, leaving a large proportion with undefined etiology.

We followed strict safeguards to control genotyping errors. We used a GWAS chip developed by Illumina, the industry leader. Our genotyping call rate was $99.9 \%$. A limitation of this study was that we were not able to assess the associations between genetic risk markers and childhood ALL risk in males and the age group 1-5 years. 
However, our interest in this study differed from the traditional approach, and we were more interested in identifying gender- and age-specific associations. We used gender and age as effect modifiers to identify gender- and age-specific associations. The case-only design is based on the independence of exposure and genotype, which means there should not be any difference in allele frequencies in two groups. Departures from this independence may affect the results. In the case of gender and age, it seems impossible to have different allele frequencies between males and females and between two age groups (1-5 years vs 6-10 years) in a healthy population. A genome-wide meta-analysis across 51 studies including overall 114,863 samples $(61,094$ women and 53,769) of European ancestry did not find any allele frequency difference at common loci between men and women $^{62}$. However, another study in four different GWAS datasets $(\mathrm{n}<165)$, identified allele frequency differences between healthy males and females in European samples ${ }^{285}$. However, none of the confounding SNPs in the second study ${ }^{285}$ showed statistical significant associations in gender-specific analysis in the present study. Such an association would be invalid due to the violation of the major assumption of case-only approach.

Issues related with case-control design do not necessarily apply to a case-only design. All of these cases were incident cases of childhood ALL and have a well-defined phenotype, with molecular ALL sub-types. However, we did not have complete data to examine associations in childhood ALL sub-types. One more strength of the present study is the validation of previously known leukemia risk markers in the parent casecontrol group. Thus, it increases the validity of our results. 


\section{CONCLUSION}

This research was conducted to explore the effect modification by gender and age of the risk for childhood ALL. A case-only study was used to examine the gender- and age-specific associations. The parent study was a case-control study, and replication of GWAS childhood ALL risk markers validated our parent data set and increased the credibility of our study. Our study identified novel risk variants for childhood ALL. These risk variants are gender- and age-specific, which means they are associated with a particular gender (males or females) or age groups (1-5 years or 6-10 years). A total of fourteen risk markers were previously yielded associations with other diseases, such as autoimmune disorders, CLL (rs7169431) and nasopharyngeal carcinoma (rs28421666).

The aim to unmask hidden gender- and age-specific risk variants was based on clear heterogeneity between two genders and age groups. There are, however, very few examples of gender- and age-specific associations in the risk of childhood ALL, but a dedicated GWAS was needed to unravel these hidden risk variants. Our results were based on two approaches: a conventional statistical analysis based on the most statistically significant associations and the results based on the highest effect sizes. The SNPs, rs4813720, rs231237 and rs7323018, yielded the statistically most significant gender-specific associations. Likewise, rs10505918 yielded the lowest P value for agespecific associations, and also yielded the most statistically significant association with age-at-onset. Two SNPs, rs 12722042 and 12722039, from the HLA-DQA1 region yielded the highest effect sizes for gender-specific results, and the SNP, rs17109582 and rs7304215, yielded the highest effect sizes for age-specific results. 
The statistical gender- or age-specific associations were complemented with bioinformatics and empirical analyses for causality assessment, and yielded some functional variants among the set of SNPs. A total of 29 SNPs showed a very high functional score for gender- and age-specific results, which suggests they may be very important for regulatory effects. Our results also identified a total of 56 nonsynonymous variations (55 missense and one nonsense) for gender- and age-specific associations; however, most of these nonsynonymous variations were associated with the results based on the effect size. These nonsynonymous variations clearly suggest an obvious change in protein function. Likewise, 21 SNPs were located in TFBS and may affect the regulatory function.

Gene enrichment analysis did not yield any specific pathways for childhood ALL risk in gender and age-specific manner. We still explored the functions of associated genes. Several of these genes are associated with RAS, PTEN, JAK/STAT and P13/AKT signaling pathways ${ }^{221,255}$. These pathways play a significant role in leukemogenesis. Overall our results yielded novel gender- and age- specific risk variants in this study. Further studies are warranted to replicate the gender- and age-specific associations and to examine mechanisms of those that are confirmed.

Markers, which showed associations differing in directions, will be very useful once confirmed risk to males and protection to females. It is important to know that because any future intervention to protect males may do harm to females if this difference is not recognized. Thus, there is potential for targeted intervention (and maybe treatment approaches) of one gender while the same intervention would not be useful to the other. These results, once confirmed, may also help to devise more personalized treatment for 
males. Just now, they get the same treatment but at a higher dose (with more side effects and late effects etc). 


\section{REFERENCES}

1. Pui CH, Robison LL, Look AT. Acute lymphoblastic leukaemia. Lancet 2008; 371(9617): 1030-1043.

2. American Cancer Society. Cancer Facts \& Figures 2014. Retrieved from: http://www.cancer.org/research/cancerfactsstatistics/cancerfactsfigures2014/.

3. Ward E, Desantis C, Robbins A, Kohler B, Jemal A. Childhood and adolescent cancer statistics, 2014. CA Cancer J Clin 2014; 64(2): 83-103.

4. $\quad$ Siegel R, Ma J, Zou Z, Jemal A. Cancer statistics, 2014. CA Cancer J Clin 2014; 64(1): 9-29.

5. National Cancer Institute, Surveillance, Epidemiology, and End Results (SEER) Program. Retrieved from:

http://seer.cancer.gov/csr/1975 2010/results_merged/sect 28 childhood cancer.p df.

6. Oeffinger KC, Mertens AC, Sklar CA, Kawashima T, Hudson MM, Meadows AT, et al. Chronic health conditions in adult survivors of childhood cancer. $N$ Engl J Med 2006; 355(15): 1572-1582.

7. Cullen J. Because statistics don't tell the whole story: A call for comprehensive care for children with cancer. CA Cancer J Clin 2014; 64(2): 79-82.

8. Mulder RL, Hudson MM, Skinner R, Kremer LC. Health problems in survivors of childhood cancer: the need for international collaboration in long-term follow-up care. Future Oncol 2013; 9(11): 1667-1670.

9. Schultz KA, Ness KK, Whitton J, Recklitis C, Zebrack B, Robison LL, et al. Behavioral and social outcomes in adolescent survivors of childhood cancer: a report from the childhood cancer survivor study. J Clin Oncol 2007; 25(24): 3649-3656.

10. Chemaitilly W, Mertens AC, Mitby P, Whitton J, Stovall M, Yasui Y, et al. Acute ovarian failure in the childhood cancer survivor study. J Clin Endocrinol Metab 2006; 91(5): 1723-1728.

11. Blanco JG, Sun CL, Landier W, Chen L, Esparza-Duran D, Leisenring W, et al. Anthracycline-related cardiomyopathy after childhood cancer: role of polymorphisms in carbonyl reductase genes--a report from the Children's Oncology Group. J Clin Oncol 2012; 30(13): 1415-1421. 
12. Mattano LA, Jr., Sather HN, Trigg ME, Nachman JB. Osteonecrosis as a complication of treating acute lymphoblastic leukemia in children: a report from the Children's Cancer Group. J Clin Oncol 2000; 18(18): 3262-3272.

13. Chow EJ, Friedman DL, Yasui Y, Whitton JA, Stovall M, Robison LL, et al. Decreased adult height in survivors of childhood acute lymphoblastic leukemia: a report from the Childhood Cancer Survivor Study. J Pediatr 2007; 150(4): 370375,375 e371.

14. Knight KR, Kraemer DF, Neuwelt EA. Ototoxicity in children receiving platinum chemotherapy: underestimating a commonly occurring toxicity that may influence academic and social development. J Clin Oncol 2005; 23(34): 8588-8596.

15. Friedman DL, Whitton J, Leisenring W, Mertens AC, Hammond S, Stovall M, et al. Subsequent neoplasms in 5-year survivors of childhood cancer: the Childhood Cancer Survivor Study. J Natl Cancer Inst 2010; 102(14): 1083-1095.

16. Bassal M, Mertens AC, Taylor L, Neglia JP, Greffe BS, Hammond S, et al. Risk of selected subsequent carcinomas in survivors of childhood cancer: a report from the Childhood Cancer Survivor Study. J Clin Oncol 2006; 24(3): 476-483.

17. Terracini B. Epidemiology of childhood cancer. Envir Health 2011; 10 Suppl 1: S8.

18. Pearce MS, Parker L. Childhood cancer registrations in the developing world: still more boys than girls. Int J Cancer 2001; 91(3): 402-406.

19. Wiemels J. Perspectives on the causes of childhood leukemia. Chem Biol Interact 2012; 196(3): 59-67.

20. Devarahally SR, Severson RK, Chuba P, Thomas R, Bhambhani K, Hamre MR. Second malignant neoplasms after primary central nervous system malignancies of childhood and adolescence. Pediat Hematol Oncol 2003; 20(8): 617-625.

21. Howard SC, Metzger ML, Wilimas JA, Quintana Y, Pui CH, Robison LL, et al. Childhood cancer epidemiology in low-income countries. Cancer 2008; 112(3): 461-472.

22. Linet MS, Wacholder S, Zahm SH. Interpreting epidemiologic research: lessons from studies of childhood cancer. Pediatrics 2003; 112(2): 218-232.

23. Pui CH, Boyett JM, Relling MV, Harrison PL, Rivera GK, Behm FG, et al. Sex differences in prognosis for children with acute lymphoblastic leukemia. J Clin Oncol 1999; 17(3): 818-824. 
24. Cook MB, Dawsey SM, Freedman ND, Inskip PD, Wichner SM, Quraishi SM, et al. Sex disparities in cancer incidence by period and age. Cancer Epidemiol Biomark Prev 2009; 18(4): 1174-1182.

25. Edgren G, Liang L, Adami HO, Chang ET. Enigmatic sex disparities in cancer incidence. Eur J Epid 2012; 27(3): 187-196.

26. Dorak MT, Karpuzoglu E. Gender differences in cancer susceptibility: an inadequately addressed issue. Front Genet 2012; 3: 268.

27. Cartwright RA, Gurney KA, Moorman AV. Sex ratios and the risks of haematological malignancies. Br J Haematol 2002; 118(4): 1071-1077.

28. Sather H, Miller D, Nesbit M, Heyn R, Hammond D. Differences in prognosis for boys and girls with acute lymphoblastic leukaemia. Lancet 1981; 1(8223): 739743.

29. Stiller CA. Epidemiology and genetics of childhood cancer. Oncogene 2004; 23(38): 6429-6444.

30. Ziegelberger G, Dehos A, Grosche B, Hornhardt S, Jung T, Weiss W. Childhood leukemia--risk factors and the need for an interdisciplinary research agenda. Prog Biophys Mol Biol 2011; 107(3): 312-314.

31. Holmes L, Jr., Hossain J, Desvignes-Kendrick M, Opara F. Sex variability in pediatric leukemia survival: large cohort evidence. ISRN Oncol 2012; 2012: 439070 .

32. Cook MB, McGlynn KA, Devesa SS, Freedman ND, Anderson WF. Sex disparities in cancer mortality and survival. Cancer Epidemiol Biomark Prev 2011; 20(8): 1629-1637.

33. Patel MI, Ma Y, Mitchell BS, Rhoads KF. Understanding disparities in leukemia: a national study. Can Caus Contr 2012; 23(11): 1831-1837.

34. Goggins WB, Lo FF. Racial and ethnic disparities in survival of US children with acute lymphoblastic leukemia: evidence from the SEER database 1988-2008. Can Caus Cont 2012; 23(5): 737-743.

35. Kadan-Lottick NS, Ness KK, Bhatia S, Gurney JG. Survival variability by race and ethnicity in childhood acute lymphoblastic leukemia. JAMA 2003; 290(15): 2008-2014.

36. Eden T. Aetiology of childhood leukaemia. Cancer Treat Rev 2010; 36(4): 286297. 
37. Reid A, Glass DC, Bailey HD, Milne E, Armstrong BK, Alvaro F, et al. Parental occupational exposure to exhausts, solvents, glues and paints, and risk of childhood leukemia. Can Caus Cont 2011; 22(11): 1575-1585.

38. Castro-Jimenez MA, Orozco-Vargas LC. Parental exposure to carcinogens and risk for childhood acute lymphoblastic leukemia, Colombia, 2000-2005. Prev Chronic Dis 2011; 8(5): A106.

39. Shu XO, Ross JA, Pendergrass TW, Reaman GH, Lampkin B, Robison LL. Parental alcohol consumption, cigarette smoking, and risk of infant leukemia: a Childrens Cancer Group study. J Natl Can Inst 1996; 88(1): 24-31.

40. Ou SX, Han D, Severson RK, Chen Z, Neglia JP, Reaman GH, et al. Birth characteristics, maternal reproductive history, hormone use during pregnancy, and risk of childhood acute lymphocytic leukemia by immunophenotype (United States). Can Caus Cont 2002; 13(1): 15-25.

41. Milne E, Royle JA, Bennett LC, de Klerk NH, Bailey HD, Bower C, et al. Maternal consumption of coffee and tea during pregnancy and risk of childhood ALL: results from an Australian case-control study. Can Caus Cont 2011; 22(2): 207-218.

42. Bailey HD, Armstrong BK, de Klerk NH, Fritschi L, Attia J, Scott RJ, et al. Exposure to professional pest control treatments and the risk of childhood acute lymphoblastic leukemia. Int J Can 2011; 129(7): 1678-1688.

43. Bailey HD, Milne E, de Klerk NH, Fritschi L, Attia J, Cole C, et al. Exposure to house painting and the use of floor treatments and the risk of childhood acute lymphoblastic leukemia. Int J Can 2011; 128(10): 2405-2414.

44. Kheifets L, Ahlbom A, Crespi CM, Draper G, Hagihara J, Lowenthal RM, et al. Pooled analysis of recent studies on magnetic fields and childhood leukaemia. $\mathrm{Br}$ J Can 2010; 103(7): 1128-1135.

45. Hrusak O, Trka J, Zuna J, Polouckova A, Kalina T, Stary J. Acute lymphoblastic leukemia incidence during socioeconomic transition: selective increase in children from 1 to 4 years. Leukemia 2002; 16(4): 720-725.

46. Downing JR, Wilson RK, Zhang J, Mardis ER, Pui CH, Ding L, et al. The Pediatric Cancer Genome Project. Nat Genet 2012; 44(6): 619-622.

47. Healthcare Cost and Utilization Project (HCUP) Statistical Briefs 132. Overview of Hospital Stays for Children in the United States, 2012 Retrieved from: http://www.hcup-us.ahrq.gov/reports/statbriefs/statbriefs.jsp 
48. Tomatis L. Poverty and cancer. IARC Sci Publ 1997; (138): 25-39.

49. Orsi L, Rudant J, Bonaventure A, Goujon-Bellec S, Corda E, Evans TJ, et al. Genetic polymorphisms and childhood acute lymphoblastic leukemia: GWAS of the ESCALE study (SFCE). Leukemia 2012; 26(12): 2561-2564.

50. Han S, Lee KM, Park SK, Lee JE, Ahn HS, Shin HY, et al. Genome-wide association study of childhood acute lymphoblastic leukemia in Korea. Leuk Res 2010; 34(10): 1271-1274.

51. Trevino LR, Yang W, French D, Hunger SP, Carroll WL, Devidas M, et al. Germline genomic variants associated with childhood acute lymphoblastic leukemia. Nat Genet 2009; 41(9): 1001-1005.

52. Papaemmanuil E, Hosking FJ, Vijayakrishnan J, Price A, Olver B, Sheridan E, et al. Loci on $7 \mathrm{p} 12.2,10 \mathrm{q} 21.2$ and $14 \mathrm{q} 11.2$ are associated with risk of childhood acute lymphoblastic leukemia. Nat Genet 2009; 41(9): 1006-1010.

53. Xu H, Yang W, Perez-Andreu V, Devidas M, Fan Y, Cheng C, et al. Novel susceptibility variants at 10p12.31-12.2 for childhood acute lymphoblastic leukemia in ethnically diverse populations. J Natl Cancer Inst 2013; 105(10): 733-742.

54. Ellinghaus E, Stanulla M, Richter G, Ellinghaus D, te Kronnie G, Cario G, et al. Identification of germline susceptibility loci in ETV6-RUNX1-rearranged childhood acute lymphoblastic leukemia. Leukemia 2012; 26(5): 902-909.

55. Enciso-Mora V, Hosking FJ, Sheridan E, Kinsey SE, Lightfoot T, Roman E, et al. Common genetic variation contributes significantly to the risk of childhood B-cell precursor acute lymphoblastic leukemia. Leukemia 2012; 26(10): 2212-2215.

56. Manolio TA, Collins FS, Cox NJ, Goldstein DB, Hindorff LA, Hunter DJ, et al. Finding the missing heritability of complex diseases. Nature 2009; 461(7265): 747-753.

57. Magi R, Lindgren CM, Morris AP. Meta-analysis of sex-specific genome-wide association studies. Genet Epidemiol 2010; 34(8): 846-853.

58. Goldstein AM, Andrieu N. Detection of interaction involving identified genes: available study designs. J Natl Cancer Inst Monogr 1999; (26): 49-54.

59. Clayton D, McKeigue PM. Epidemiological methods for studying genes and environmental factors in complex diseases. Lancet 2001; 358(9290): 1356-1360. 
60. Piegorsch WW, Weinberg CR, Taylor JA. Non-hierarchical logistic models and case-only designs for assessing susceptibility in population-based case-control studies. Stat Med 1994; 13(2): 153-162.

61. Khoury MJ, Flanders WD. Nontraditional epidemiologic approaches in the analysis of gene-environment interaction: case-control studies with no controls! Am J Epidemiol 1996; 144(3): 207-213.

62. Boraska V, Jeroncic A, Colonna V, Southam L, Nyholt DR, Rayner NW, et al. Genome-wide meta-analysis of common variant differences between men and women. Hum Mol Genet 2012; 21(21): 4805-4815.

63. Liu X, Fallin MD, Kao WH. Genetic dissection methods: designs used for tests of gene-environment interaction. Curr Opin Genet Dev 2004; 14(3): 241-245.

64. Pierce BL, Ahsan H. Case-only genome-wide interaction study of disease risk, prognosis and treatment. Genet Epidemiol 2010; 34(1): 7-15.

65. Hamajima N, Yuasa H, Matsuo K, Kurobe Y. Detection of gene-environment interaction by case-only studies. Jpn J Clin Oncol 1999; 29(10): 490-493.

66. Magrath I, Steliarova-Foucher E, Epelman S, Ribeiro RC, Harif M, Li CK, et al. Paediatric cancer in low-income and middle-income countries. Lancet Oncol 2013; 14(3): e104-116.

67. National Cancer Institute. An Analysis of the National Cancer Institute's Investment in Pediatric Cancer Research. Retrieved from: http://www.cancer.gov/researchandfunding/reports/pediatric-analysis.pdf.

68. Liu LY, Schaub MA, Sirota M, Butte AJ. Sex differences in disease risk from reported genome-wide association study findings. Hum Genet 2012; 131(3): 353364.

69. Ashley DJ. A male-female differential in tumour incidence. Br J Cancer 1969; 23(1): 21-25.

70. Loisel DA, Tan Z, Tisler CJ, Evans MD, Gangnon RE, Jackson DJ, et al. IFNG genotype and sex interact to influence the risk of childhood asthma. J Allergy Clin Immunol 2011; 128(3): 524-531.

71. Kantarci OH, Goris A, Hebrink DD, Heggarty S, Cunningham S, Alloza I, et al. IFNG polymorphisms are associated with gender differences in susceptibility to multiple sclerosis. Genes Immun 2005; 6(2): 153-161. 
72. Shifman S, Johannesson M, Bronstein M, Chen SX, Collier DA, Craddock NJ, et al. Genome-wide association identifies a common variant in the reelin gene that increases the risk of schizophrenia only in women. PLoS Genet 2008; 4(2): e28.

73. Goes FS, Willour VL, Zandi PP, Belmonte PL, MacKinnon DF, Mondimore FM, et al. Sex-specific association of the Reelin gene with bipolar disorder. Am J Med Genet B Neuropsychiatr Genet 2010; 153B(2): 549-553.

74. Doring A, Gieger C, Mehta D, Gohlke H, Prokisch H, Coassin S, et al. SLC2A9 influences uric acid concentrations with pronounced sex-specific effects. Nat Genet 2008; 40(4): 430-436.

75. Corder EH, Saunders AM, Strittmatter WJ, Schmechel DE, Gaskell PC, Small $\mathrm{GW}$, et al. Gene dose of apolipoprotein E type 4 allele and the risk of Alzheimer's disease in late onset families. Science 1993; 261(5123): 921-923.

76. Chartier-Harlin MC, Crawford F, Houlden H, Warren A, Hughes D, Fidani L, et al. Early-onset Alzheimer's disease caused by mutations at codon 717 of the betaamyloid precursor protein gene. Nature 1991; 353(6347): 844-846.

77. Guo Z, Yang H, Zhang F, Zhang R, Wang C. Single nucleotide polymorphisms in the mitochondrial displacement loop and age-at-onset of esophageal squamous cell carcinoma. Oncol Lett 2012; 3(2): 482-484.

78. Espino-Paisan L, de la Calle H, Fernandez-Arquero M, Figueredo MA, de la Concha EG, Urcelay E, et al. A polymorphism in PTPN2 gene is associated with an earlier onset of type 1 diabetes. Immunogenetics 2011; 63(4): 255-258.

79. National Institute of Health. Policy for sharing of data obtained in NIH supported or conducted genome-wide association studies (GWAS). Retrieved from: http://grants.nih.giv/grants/guide/notice-files/NOT-OD-07-088.html

80. Pearson TA, Manolio TA. How to interpret a genome-wide association study. JAMA 2008; 299(11): 1335-1344.

81. Weale ME. Quality control for genome-wide association studies. Methods Mol Biol 2010; 628: 341-372.

82. Anderson CA, Pettersson FH, Clarke GM, Cardon LR, Morris AP, Zondervan KT. Data quality control in genetic case-control association studies. Nat Protoc 2010; 5(9): 1564-1573.

83. Slagboom PE, Meulenbelt I. Organisation of the human genome and our tools for identifying disease genes. Biol Psychol 2002; 61(1-2): 11-31. 
84. ENCODE. Statistics about the current GENCODE freeze (version 21). Retrieved from: http://www.gencodegenes.org/stats.html

85. Ellegren H, Parsch J. The evolution of sex-biased genes and sex-biased gene expression. Nat Rev Genet 2007; 8(9): 689-698.

86. Ober C, Loisel DA, Gilad Y. Sex-specific genetic architecture of human disease. Nat Rev Genet 2008; 9(12): 911-922.

87. Restif O, Amos W. The evolution of sex-specific immune defences. Proc Biol Sci 2010; 277(1691): 2247-2255.

88. Woosley RL, Anthony M, Peck CC. Biological sex analysis in clinical research. $J$ Womens Health Gend Based Med 2000; 9(9): 933-934.

89. Yeh SH, Chen PJ. Gender disparity of hepatocellular carcinoma: the roles of sex hormones. Oncology 2010; 78 Suppl 1: 172-179.

90. Naugler WE, Sakurai T, Kim S, Maeda S, Kim K, Elsharkawy AM, et al. Gender disparity in liver cancer due to sex differences in MyD88-dependent IL-6 production. Science 2007; 317(5834): 121-124.

91. Lawrence T, Hageman T, Balkwill F. Cancer. Sex, cytokines, and cancer. Science 2007; 317(5834): 51-52.

92. Weige CC, Allred KF, Allred CD. Estradiol alters cell growth in nonmalignant colonocytes and reduces the formation of preneoplastic lesions in the colon. Cancer Res 2009; 69(23): 9118-9124.

93. Rahbari R, Zhang L, Kebebew E. Thyroid cancer gender disparity. Future Oncol 2010; 6(11): 1771-1779.

94. Stanley JA, Aruldhas MM, Chandrasekaran M, Neelamohan R, Suthagar E, Annapoorna $\mathrm{K}$, et al. Androgen receptor expression in human thyroid cancer tissues: a potential mechanism underlying the gender bias in the incidence of thyroid cancers. J Steroid Biochem Mol Biol 2012; 130(1-2): 105-124.

95. McMillen MM. Differential mortality by sex in fetal and neonatal deaths. Science 1979; 204(4388): 89-91.

96. Libert C, Dejager L, Pinheiro I. The X chromosome in immune functions: when a chromosome makes the difference. Nat Rev Immunol 2010; 10(8): 594-604. 
97. Glezen WP, Loda FA, Clyde WA, Jr., Senior RJ, Sheaffer CI, Conley WG, et al. Epidemiologic patterns of acute lower respiratory disease of children in a pediatric group practice. J Pediatr 1971; 78(3): 397-406.

98. Hall CB, Kopelman AE, Douglas RG, Jr., Geiman JM, Meagher MP. Neonatal respiratory syncytial virus infection. $N$ Engl J Med 1979; 300(8): 393-396.

99. Thompson DJ, Gezon HM, Rogers KD, Yee RB, Hatch TF. Excess risk of staphylococcal infection and disease in newborn males. Am J Epidemiol 1966; 84(2): 314-328.

100. Strachan NJ, Watson RO, Novik V, Hofreuter D, Ogden ID, Galan JE. Sexual dimorphism in campylobacteriosis. Epidemiol Infect 2008; 136(11): 1492-1495.

101. Green MS. The male predominance in the incidence of infectious diseases in children: a postulated explanation for disparities in the literature. Int J Epidemiol 1992; 21(2): 381-386.

102. Muenchhoff M, Goulder PJ. Sex differences in pediatric infectious diseases. $J$ Infect Dis 2014; 209 Suppl 3: S120-126.

103. van Lunzen J, Altfeld M. Sex differences in infectious diseases-common but neglected. J Infect Dis 2014; 209 Suppl 3: S79-80.

104. Migeon BR. The role of X inactivation and cellular mosaicism in women's health and sex-specific diseases. JAMA 2006; 295(12): 1428-1433.

105. Aspinall R. Longevity and the immune response. Biogerontology 2000; 1(3): 273278.

106. Choudhry MA, Bland KI, Chaudry IH. Gender and susceptibility to sepsis following trauma. Endocr Metab Immune Disord Drug Targets 2006; 6(2): 127 135.

107. Gannon CJ, Pasquale M, Tracy JK, McCarter RJ, Napolitano LM. Male gender is associated with increased risk for postinjury pneumonia. Shock 2004; 21(5): 410414.

108. Klein SL. Immune cells have sex and so should journal articles. Endocrinology 2012; 153(6): 2544-2550.

109. Grossman C. Possible underlying mechanisms of sexual dimorphism in the immune response, fact and hypothesis. J Steroid Biochem 1989; 34(1-6): 241251. 
110. Ngo ST, Steyn FJ, McCombe PA. Gender differences in autoimmune disease. Front Neuroendocrinol 2014; 35(3): 347-369.

111. Purtilo DT, Sullivan JL. Immunological bases for superior survival of females. Am J Dis Child 1979; 133(12): 1251-1253.

112. Liebert MA. Exploring the biological contributions to human health: does sex matter? J Womens Health Gend Based Med 2001; 10(5): 433-439.

113. Austad SN. Why women live longer than men: sex differences in longevity. Gend Med 2006; 3(2): 79-92.

114. Rinn JL, Snyder M. Sexual dimorphism in mammalian gene expression. Trends Genet 2005; 21(5): 298-305.

115. Ostrer H. Invited review: sex-based differences in gene expression. J Appl Physiol (1985) 2001; 91(5): 2384-2388.

116. Mank JE, Nam K, Brunstrom B, Ellegren H. Ontogenetic complexity of sexual dimorphism and sex-specific selection. Mol Biol Evol 2010; 27(7): 1570-1578.

117. Arnold AP, Lusis AJ. Understanding the sexome: measuring and reporting sex differences in gene systems. Endocrinology 2012; 153(6): 2551-2555.

118. Dimas AS, Nica AC, Montgomery SB, Stranger BE, Raj T, Buil A, et al. Sexbiased genetic effects on gene regulation in humans. Genome Res 2012; 22(12): 2368-2375.

119. Yang X, Schadt EE, Wang S, Wang H, Arnold AP, Ingram-Drake L, et al. Tissuespecific expression and regulation of sexually dimorphic genes in mice. Genome Res 2006; 16(8): 995-1004.

120. Reinke V, Gil IS, Ward S, Kazmer K. Genome-wide germline-enriched and sexbiased expression profiles in Caenorhabditis elegans. Development 2004; 131(2): $311-323$.

121. Ranz JM, Castillo-Davis CI, Meiklejohn CD, Hartl DL. Sex-dependent gene expression and evolution of the Drosophila transcriptome. Science 2003; 300(5626): 1742-1745.

122. Do TN, Ucisik-Akkaya E, Davis CF, Morrison BA, Dorak MT. An intronic polymorphism of IRF4 gene influences gene transcription in vitro and shows a risk association with childhood acute lymphoblastic leukemia in males. Biochim Biophys Acta 2010; 1802(2): 292-300. 
123. Dorak MT, Lawson T, Machulla HK, Darke C, Mills KI, Burnett AK. Unravelling an HLA-DR association in childhood acute lymphoblastic leukemia. Blood 1999; 94(2): 694-700.

124. Dorak MT, Burnett AK, Worwood M, Sproul AM, Gibson BE. The C282Y mutation of HFE is another male-specific risk factor for childhood acute lymphoblastic leukemia. Blood 1999; 94(11): 3957.

125. Dorak MT, Oguz FS, Yalman N, Diler AS, Kalayoglu S, Anak S, et al. A malespecific increase in the HLA-DRB4 (DR53) frequency in high-risk and relapsed childhood ALL. Leuk Res 2002; 26(7): 651-656.

126. Do TN, Ucisik-Akkaya E, Davis CF, Morrison BA, Dorak MT. TP53 R72P and MDM2 SNP309 polymorphisms in modification of childhood acute lymphoblastic leukemia susceptibility. Cancer Genet Cytogenet 2009; 195(1): 3136.

127. Dorak MT, Mackay RK, Relton CL, Worwood M, Parker L, Hall AG. Hereditary hemochromatosis gene (HFE) variants are associated with birth weight and childhood leukemia risk. Pediatr Blood Cancer 2009; 53(7): 1242-1248.

128. Davis CF, Dorak MT. An extensive analysis of the hereditary hemochromatosis gene HFE and neighboring histone genes: associations with childhood leukemia. Ann Hematol 2010; 89(4): 375-384.

129. Ucisik-Akkaya E, Davis CF, Gorodezky C, Alaez C, Dorak MT. HLA complexlinked heat shock protein genes and childhood acute lymphoblastic leukemia susceptibility. Cell Stress Chaperones 2010; 15(5): 475-485.

130. Morrison BA, Ucisik-Akkaya E, Flores H, Alaez C, Gorodezky C, Dorak MT. Multiple sclerosis risk markers in HLA-DRA, HLA-C, and IFNG genes are associated with sex-specific childhood leukemia risk. Autoimmunity 2010; 43(8): 690-697.

131. Kennedy AE, Kamdar KY, Lupo PJ, Okcu MF, Scheurer ME, Dorak MT. Genetic markers in a multi-ethnic sample for childhood acute lymphoblastic leukemia risk. Leuk Lymphoma 2014: 1-6.

132. Watson MA, Gay L, Stebbings WS, Speakman CT, Bingham SA, Loktionov A. Apolipoprotein E gene polymorphism and colorectal cancer: gender-specific modulation of risk and prognosis. Clin Sci (Lond) 2003; 104(5): 537-545.

133. Zheng L, Wang Y, Schabath MB, Grossman HB, Wu X. Sulfotransferase 1A1 (SULT1A1) polymorphism and bladder cancer risk: a case-control study. Cancer Lett 2003; 202(1): 61-69. 
134. Bond GL, Hu W, Bond EE, Robins H, Lutzker SG, Arva NC, et al. A single nucleotide polymorphism in the MDM2 promoter attenuates the p53 tumor suppressor pathway and accelerates tumor formation in humans. Cell 2004; 119(5): 591-602.

135. Bolufer P, Collado M, Barragan E, Cervera J, Calasanz MJ, Colomer D, et al. The potential effect of gender in combination with common genetic polymorphisms of drug-metabolizing enzymes on the risk of developing acute leukemia.

Haematologica 2007; 92(3): 308-314.

136. Suzuki H, Morris JS, Li Y, Doll MA, Hein DW, Liu J, et al. Interaction of the cytochrome P4501A2, SULT1A1 and NAT gene polymorphisms with smoking and dietary mutagen intake in modification of the risk of pancreatic cancer. Carcinogenesis 2008; 29(6): 1184-1191.

137. Chen D, Truong T, Gaborieau V, Byrnes G, Chabrier A, Chuang SC, et al. A sexspecific association between a 15q25 variant and upper aerodigestive tract cancers. Cancer Epidemiol Biomarkers Prev 2011; 20(4): 658-664.

138. Vineis P, Veglia F, Benhamou S, Butkiewicz D, Cascorbi I, Clapper ML, et al. CYP1A1 T3801 C polymorphism and lung cancer: a pooled analysis of 2451 cases and 3358 controls. Int J Cancer 2003; 104(5): 650-657.

139. Healy J, Richer C, Bourgey M, Kritikou EA, Sinnett D. Replication analysis confirms the association of ARID5B with childhood B-cell acute lymphoblastic leukemia. Haematologica 2010; 95(9): 1608-1611.

140. Gorniak P, Pastorczak A, Zalewska-Szewczyk B, Lejman M, Trelinska J, Chmielewska M, et al. Polymorphism in IKZF1 gene affects age at onset of childhood acute lymphoblastic leukemia. Leuk Lymphoma 2014; 55(9): 21742178 .

141. Liu LY, Schaub MA, Sirota M, Butte AJ. Transmission distortion in Crohn's disease risk gene ATG16L1 leads to sex difference in disease association. Inflamm Bowel Dis 2012; 18(2): 312-322.

142. Baggio G, Corsini A, Floreani A, Giannini S, Zagonel V. Gender medicine: a task for the third millennium. Clin Chem Lab Med 2013; 51(4): 713-727.

143. Ebert SN, Liu XK, Woosley RL. Female gender as a risk factor for drug-induced cardiac arrhythmias: evaluation of clinical and experimental evidence. $J$ Womens Health 1998; 7(5): 547-557. 
144. Martin RM, Biswas PN, Freemantle SN, Pearce GL, Mann RD. Age and sex distribution of suspected adverse drug reactions to newly marketed drugs in general practice in England: analysis of 48 cohort studies. Br J Clin Pharmacol 1998; 46(5): 505-511.

145. Duma D, Collins JB, Chou JW, Cidlowski JA. Sexually dimorphic actions of glucocorticoids provide a link to inflammatory diseases with gender differences in prevalence. Sci Signal 2010; 3(143): ra74.

146. Mittelstrass K, Ried JS, Yu Z, Krumsiek J, Gieger C, Prehn C, et al. Discovery of sexual dimorphisms in metabolic and genetic biomarkers. PLoS Genet 2011; 7(8): e1002215.

147. Cookson W, Liang L, Abecasis G, Moffatt M, Lathrop M. Mapping complex disease traits with global gene expression. Nat Rev Genet 2009; 10(3): 184-194.

148. Nica AC, Dermitzakis ET. Using gene expression to investigate the genetic basis of complex disorders. Hum Mol Genet 2008; 17(R2): R129-134.

149. Kong A, Thorleifsson G, Stefansson H, Masson G, Helgason A, Gudbjartsson DF, et al. Sequence variants in the RNF212 gene associate with genome-wide recombination rate. Science 2008; 319(5868): 1398-1401.

150. Williams RB, Marchuk DA, Gadde KM, Barefoot JC, Grichnik K, Helms MJ, et al. Serotonin-related gene polymorphisms and central nervous system serotonin function. Neuropsychopharmacology 2003; 28(3): 533-541.

151. Coviello AD, Haring R, Wellons M, Vaidya D, Lehtimaki T, Keildson S, et al. A genome-wide association meta-analysis of circulating sex hormone-binding globulin reveals multiple Loci implicated in sex steroid hormone regulation. PLoS Genet 2012; 8(7): e1002805.

152. Lindgren CM, Heid IM, Randall JC, Lamina C, Steinthorsdottir V, Qi L, et al. Genome-wide association scan meta-analysis identifies three Loci influencing adiposity and fat distribution. PLoS Genet 2009; 5(6): e1000508.

153. Fish EN. The X-files in immunity: sex-based differences predispose immune responses. Nat Rev Immunol 2008; 8(9): 737-744.

154. Swinney RM, Hsu SC, Hirschman BA, Chen TT, Tomlinson GE. MDM2 promoter variation and age of diagnosis of acute lymphoblastic leukemia. Leukemia 2005; 19(11): 1996-1998.

155. Yang Y, Li T, Nielsen ME. Aging and cancer mortality: dynamics of change and sex differences. Exp Gerontol 2012; 47(9): 695-705. 
156. Frank SA. Genetic predisposition to cancer - insights from population genetics. Nat Rev Genet 2004; 5(10): 764-772.

157. Bougeard G, Baert-Desurmont S, Tournier I, Vasseur S, Martin C, Brugieres L, et al. Impact of the MDM2 SNP309 and p53 Arg72Pro polymorphism on age of tumour onset in Li-Fraumeni syndrome. J Med Genet 2006; 43(6): 531-533.

158. Raynor LA, Pankratz N, Spector LG. An analysis of measures of effect size by age of onset in cancer genomewide association studies. Genes Chromosomes Cancer 2013; 52(9): 855-859.

159. Agopian AJ, Eastcott LM, Mitchell LE. Age of onset and effect size in genomewide association studies. Birth Defects Res A Clin Mol Teratol 2012; 94(11): 908911.

160. Schmidt S, Barcellos LF, DeSombre K, Rimmler JB, Lincoln RR, Bucher P, et al. Association of polymorphisms in the apolipoprotein $\mathrm{E}$ region with susceptibility to and progression of multiple sclerosis. Am J Hum Genet 2002; 70(3): 708-717.

161. Mahley RW, Weisgraber KH, Huang Y. Apolipoprotein E4: a causative factor and therapeutic target in neuropathology, including Alzheimer's disease. Proc Natl Acad Sci U S A 2006; 103(15): 5644-5651.

162. Wisniewski T, Frangione B. Apolipoprotein E: a pathological chaperone protein in patients with cerebral and systemic amyloid. Neurosci Lett 1992; 135(2): 235238.

163. Tamam Y, Tasdemir N, Yalman M, Tamam B. Association of apolipoprotein E genotypes with prognosis in multiple sclerosis. Eur Rev Med Pharmacol Sci 2011; 15(10): 1122-1130.

164. Huang R, Hughes M, Mobley S, Lanham I, Poduslo SE. APOE genotypes in African American female multiple sclerosis patients. Neurosci Lett 2007; 414(1): 51-56.

165. Sadeghi HM, Sabzghabaee AM, Mousavian Z, Saadatnia M, Shirani S, Moazen F. Polymorphism of Apo lipoprotein E gene and the risk of multiple sclerosis. J Res Med Sci 2011; 16(12): 1519-1524.

166. Bond GL, Levine AJ. A single nucleotide polymorphism in the p53 pathway interacts with gender, environmental stresses and tumor genetics to influence cancer in humans. Oncogene 2007; 26(9): 1317-1323. 
167. Bond GL, Hirshfield KM, Kirchhoff T, Alexe G, Bond EE, Robins H, et al. MDM2 SNP309 accelerates tumor formation in a gender-specific and hormonedependent manner. Cancer Res 2006; 66(10): 5104-5110.

168. Bond GL, Menin C, Bertorelle R, Alhopuro P, Aaltonen LA, Levine AJ. MDM2 SNP309 accelerates colorectal tumour formation in women. J Med Genet 2006; 43(12): 950-952.

169. Kulkarni DA, Vazquez A, Haffty BG, Bandera EV, Hu W, Sun YY, et al. A polymorphic variant in human MDM4 associates with accelerated age of onset of estrogen receptor negative breast cancer. Carcinogenesis 2009; 30(11): 19101915.

170. Oguz FS, Kalayoglu S, Diler AS, Tozkir H, Sargin D, Carin M, et al. HLA system affects the age-at-onset in chronic myeloid leukemia. Am J Hematol 2003; 73(4): 256-262.

171. Shi Z, Johnstone D, Talseth-Palmer BA, Evans TJ, Spigelman AD, Groombridge $\mathrm{C}$, et al. Haemochromatosis HFE gene polymorphisms as potential modifiers of hereditary nonpolyposis colorectal cancer risk and onset age. Int J Cancer 2009; 125(1): 78-83.

172. Verdonk P, Klinge I. Mainstreaming sex and gender analysis in public health genomics. Gend Med 2012; 9(6): 402-410.

173. Purcell S, Neale B, Todd-Brown K, Thomas L, Ferreira MA, Bender D, et al. PLINK: a tool set for whole-genome association and population-based linkage analyses. Am J Hum Genet 2007; 81(3): 559-575.

174. Laurie CC, Doheny KF, Mirel DB, Pugh EW, Bierut LJ, Bhangale T, et al. Quality control and quality assurance in genotypic data for genome-wide association studies. Genet Epidemiol 2010; 34(6): 591-602.

175. Renteria ME, Cortes A, Medland SE. Using PLINK for Genome-Wide Association Studies (GWAS) and data analysis. Methods Mol Biol 2013; 1019: 193-213.

176. Turner S, Armstrong LL, Bradford Y, Carlson CS, Crawford DC, Crenshaw AT, et al. Quality control procedures for genome-wide association studies. Curr Protoc Hum Genet 2011; Chapter 1: Unit1 19.

177. Ziegler A. Genome-wide association studies: quality control and population-based measures. Genet Epidemiol 2009; 33 Suppl 1: S45-50. 
178. Devlin B, Roeder K. Genomic control for association studies. Biometrics 1999; 55(4): 997-1004.

179. Devlin B, Bacanu SA, Roeder K. Genomic Control to the extreme. Nat Genet 2004; 36(11): 1129-1130.

180. Becker T, Cichon S, Jonson E, Knapp M. Multiple testing in the context of haplotype analysis revisited: application to case-control data. Ann Hum Genet 2005; 69(6): 747-756.

181. Lewis CM, Knight J. Introduction to genetic association studies. Cold Spring Harb Protoc 2012; 2012(3): 297-306.

182. Goel MK, Khanna P, Kishore J. Understanding survival analysis: Kaplan-Meier estimate. Int J Ayurveda Res 2010; 1(4): 274-278.

183. Bewick V, Cheek L, Ball J. Statistics review 12: survival analysis. Crit Care 2004; 8(5): 389-394.

184. Boyle AP, Hong EL, Hariharan M, Cheng Y, Schaub MA, Kasowski M, et al. Annotation of functional variation in personal genomes using RegulomeDB. Genome Res 2012; 22(9): 1790-1797.

185. Lee PH, Shatkay H. F-SNP: computationally predicted functional SNPs for disease association studies. Nucleic Acids Res 2008; 36: D820-824.

186. Gamazon ER, Zhang W, Konkashbaev A, Duan S, Kistner EO, Nicolae DL, et al. SCAN: SNP and copy number annotation. Bioinformatics 2010; 26(2): 259-262.

187. Ramos EM, Hoffman D, Junkins HA, Maglott D, Phan L, Sherry ST, et al. Phenotype-Genotype Integrator (PheGenI): synthesizing genome-wide association study (GWAS) data with existing genomic resources. Eur J Hum Genet 2014; 22(1): 144-147.

188. Dayem Ullah AZ, Lemoine NR, Chelala C. A practical guide for the functional annotation of genetic variations using SNPnexus. Brief Bioinform 2013; 14(4): 437-447.

189. Ashburner M, Ball CA, Blake JA, Botstein D, Butler H, Cherry JM, et al. Gene ontology: tool for the unification of biology. The Gene Ontology Consortium. Nat Genet 2000; 25(1): 25-29.

190. Davis AP, Murphy CG, Johnson R, Lay JM, Lennon-Hopkins K, SaraceniRichards C, et al. The Comparative Toxicogenomics Database: update 2013. Nucleic Acids Res 2013; 41: D1104-1114. 
191. International Agency for Research on Cancer IARC Monographs on the Evaluation of Carcinogen Risks to Humans. Retrieved from: http://monographs.iarc.fr/ENG/Classification/

192. Ma X, Buffler PA, Gunier RB, Dahl G, Smith MT, Reinier K, et al. Critical windows of exposure to household pesticides and risk of childhood leukemia. Environ Health Perspect 2002; 110(9): 955-960.

193. Ferreira JD, Couto AC, Pombo-de-Oliveira MS, Koifman S. In utero pesticide exposure and leukemia in Brazilian children $<2$ years of age. Environ Health Perspect 2013; 121(2): 269-275.

194. Ward MH, Colt JS, Metayer C, Gunier RB, Lubin J, Crouse V, et al. Residential exposure to polychlorinated biphenyls and organochlorine pesticides and risk of childhood leukemia. Environ Health Perspect 2009; 117(6): 1007-1013.

195. Zhou Y, Zhang S, Li Z, Zhu J, Bi Y, Bai Y, et al. Maternal benzene exposure during pregnancy and risk of childhood acute lymphoblastic leukemia: a metaanalysis of epidemiologic studies. PLoS One 2014; 9(10): e110466.

196. Heck JE, Park AS, Qiu J, Cockburn M, Ritz B. Risk of leukemia in relation to exposure to ambient air toxics in pregnancy and early childhood. Int J Hyg Environ Health 2014; 217(6): 662-668.

197. Corti M, Snyder CA. Influences of gender, development, pregnancy and ethanol consumption on the hematotoxicity of inhaled $10 \mathrm{ppm}$ benzene. Arch Toxicol 1996; 70(3-4): 209-217.

198. Kenyon EM, Kraichely RE, Hudson KT, Medinsky MA. Differences in rates of benzene metabolism correlate with observed genotoxicity. Toxicol Appl Pharmacol 1996; 136(1): 49-56.

199. Kasner EJ, Keralis JM, Mehler L, Beckman J, Bonnar-Prado J, Lee SJ, et al. Gender differences in acute pesticide-related illnesses and injuries among farmworkers in the United States, 1998-2007. Am J Ind Med 2012; 55(7): 571583.

200. Garcia AM. Pesticide exposure and women's health. Am J Ind Med 2003; 44(6): 584-594.

201. Johnson KE, McMorris BJ, Raynor LA, Monsen KA. What big size you have! Using effect sizes to determine the impact of public health nursing interventions. Appl Clin Inform 2013; 4(3): 434-444. 
202. Sullivan GM, Feinn R. Using Effect Size-or Why the P Value Is Not Enough. $J$ Grad Med Educ 2012; 4(3): 279-282.

203. Crowther-Swanepoel D, Broderick P, Di Bernardo MC, Dobbins SE, Torres M, Mansouri M, et al. Common variants at 2q37.3, 8q24.21, 15q21.3 and 16q24.1 influence chronic lymphocytic leukemia risk. Nat Genet 2010; 42(2): 132-136.

204. Bei JX, Li Y, Jia WH, Feng BJ, Zhou G, Chen LZ, et al. A genome-wide association study of nasopharyngeal carcinoma identifies three new susceptibility loci. Nat Genet 2010; 42(7): 599-603.

205. Howson JM, Walker NM, Clayton D, Todd JA. Confirmation of HLA class II independent type 1 diabetes associations in the major histocompatibility complex including HLA-B and HLA-A. Diabetes Obes Metab 2009; 11 (1): 31-45.

206. Taylor GM, Dearden S, Ravetto P, Ayres M, Watson P, Hussain A, et al. Genetic susceptibility to childhood common acute lymphoblastic leukaemia is associated with polymorphic peptide-binding pocket profiles in HLA-DPB1*0201. Hum Mol Genet 2002; 11(14): 1585-1597.

207. Taylor GM, Dearden S, Payne N, Ayres M, Gokhale DA, Birch JM, et al. Evidence that an HLA-DQA1-DQB1 haplotype influences susceptibility to childhood common acute lymphoblastic leukaemia in boys provides further support for an infection-related aetiology. Br J Cancer 1998; 78(5): 561-565.

208. Taylor GM, Hussain A, Lightfoot TJ, Birch JM, Eden TO, Greaves MF. HLAassociated susceptibility to childhood B-cell precursor ALL: definition and role of HLA-DPB1 supertypes. Br J Cancer 2008; 98(6): 1125-1131.

209. Barrett JC, Clayton DG, Concannon P, Akolkar B, Cooper JD, Erlich HA, et al. Genome-wide association study and meta-analysis find that over 40 loci affect risk of type 1 diabetes. Nat Genet 2009; 41(6): 703-707.

210. Baranzini SE, Wang J, Gibson RA, Galwey N, Naegelin Y, Barkhof F, et al. Genome-wide association analysis of susceptibility and clinical phenotype in multiple sclerosis. Hum Mol Genet 2009; 18(4): 767-778.

211. Huffmeier U, Uebe S, Ekici AB, Bowes J, Giardina E, Korendowych E, et al. Common variants at TRAF3IP2 are associated with susceptibility to psoriatic arthritis and psoriasis. Nat Genet 2010; 42(11): 996-999.

212. McGovern DP, Taylor KD, Landers C, Derkowski C, Dutridge D, Dubinsky M, et al. MAGI2 genetic variation and inflammatory bowel disease. Inflamm Bowel Dis 2009; 15(1): 75-83. 
213. Bernard SM, Cartwright RA, Darwin CM, Richards ID, Roberts B, O'Brien C, et al. A possible epidemiological association between multiple sclerosis and lymphoma/leukaemia. Br J Haematol 1987; 65(1): 122-123.

214. Buckley JD, Gilchrist GS, Ruccione K, Sather HN, Woods WG, Hammond GD. Multiple sclerosis in mothers of children with acute lymphoblastic leukemia. Leukemia 1989; 3(10): 736-739.

215. Till M, Rapson N, Smith PG. Family studies in acute leukaemia in childhood: a possible association with autoimmune disease. Br J Cancer 1979; 40(1): 62-71.

216. Perillat-Menegaux F, Clavel J, Auclerc MF, Baruchel A, Leverger G, Nelken B, et al. Family history of autoimmune thyroid disease and childhood acute leukemia. Cancer Epidemiol Biomark Prev 2003; 12(1): 60-63.

217. Hemminki K, Li X, Sundquist J, Sundquist K. Cancer risks in Crohn disease patients. Ann Oncol 2009; 20(3): 574-580.

218. Hemminki K, Houlston R, Sundquist J, Sundquist K, Shu X. Co-morbidity between early-onset leukemia and type 1 diabetes--suggestive of a shared viral etiology? PLoS One 2012; 7(6): e39523.

219. Yang JJ, Cheng C, Devidas M, Cao X, Campana D, Yang W, et al. Genome-wide association study identifies germline polymorphisms associated with relapse of childhood acute lymphoblastic leukemia. Blood 2012; 120(20): 4197-4204.

220. Li J, Zhai X, Wang H, Qian X, Miao H, Zhu X. Bioinformatics analysis of gene expression profiles in childhood B-precursor acute lymphoblastic leukemia. Hematology 2014.

221. Steelman LS, Abrams SL, Whelan J, Bertrand FE, Ludwig DE, Basecke J, et al. Contributions of the Raf/MEK/ERK, PI3K/PTEN/Akt/mTOR and Jak/STAT pathways to leukemia. Leukemia 2008; 22(4): 686-707.

222. Gutierrez A, Sanda T, Grebliunaite R, Carracedo A, Salmena L, Ahn Y, et al. High frequency of PTEN, PI3K, and AKT abnormalities in T-cell acute lymphoblastic leukemia. Blood 2009; 114(3): 647-650.

223. Gomes AM, Soares MV, Ribeiro P, Caldas J, Povoa V, Martins LR, et al. Adult B-cell acute lymphoblastic leukemia cells display decreased PTEN activity and constitutive hyperactivation of PI3K/Akt pathway despite high PTEN protein levels. Haematologica 2014; 99(6): 1062-1068. 
224. Dorak MT, Pearce MS, Hammal DM, McNally RJ, Parker L. Examination of gender effect in birth weight and miscarriage associations with childhood cancer (United Kingdom). Can Caus Cont 2007; 18(2): 219-228.

225. Hanna CW, Bretherick KL, Liu CC, Stephenson MD, Robinson WP. Genetic variation within the hypothalamus-pituitary-ovarian axis in women with recurrent miscarriage. Hum Reprod 2010; 25(10): 2664-2671.

226. Ferreira M, Mesquita M, Quaresma M, Andre S. Prolactin receptor expression in gynaecomastia and male breast carcinoma. Histopathology 2008; 53(1): 56-61.

227. Wang SL, Zhao H, Zhou B, Chen YL, Zou Y, Zhu XF, et al. Polymorphisms in ERCC1 and susceptibility to childhood acute lymphoblastic leukemia in a Chinese population. Leuk Res 2006; 30(11): 1341-1345.

228. El Omari K, Hoosdally SJ, Tuladhar K, Karia D, Vyas P, Patient R, et al. Structure of the leukemia oncogene LMO2: implications for the assembly of a hematopoietic transcription factor complex. Blood 2011; 117(7): 2146-2156.

229. Cobanoglu U, Sonmez M, Ozbas HM, Erkut N, Can G. The expression of LMO2 protein in acute B-cell and myeloid leukemia. Hematology 2010; 15(3): 132-134.

230. Shams TM. High expression of LMO2 in Hodgkin, Burkitt and germinal center diffuse large B cell lymphomas. J Egypt Natl Canc Inst 2011; 23(4): 147-153.

231. McCormack MP, Young LF, Vasudevan S, de Graaf CA, Codrington R, Rabbitts $\mathrm{TH}$, et al. The Lmo2 oncogene initiates leukemia in mice by inducing thymocyte self-renewal. Science 2010; 327(5967): 879-883.

232. Nam CH, Rabbitts TH. The role of LMO2 in development and in T cell leukemia after chromosomal translocation or retroviral insertion. Mol Ther 2006; 13(1): 1525 .

233. Pike-Overzet K, de Ridder D, Weerkamp F, Baert MR, Verstegen MM, Brugman $\mathrm{MH}$, et al. Ectopic retroviral expression of LMO2, but not IL2Rgamma, blocks human T-cell development from CD34+ cells: implications for leukemogenesis in gene therapy. Leukemia 2007; 21(4): 754-763.

234. Malumbres R, Fresquet V, Roman-Gomez J, Bobadilla M, Robles EF, Altobelli $\mathrm{GG}$, et al. LMO2 expression reflects the different stages of blast maturation and genetic features in B-cell acute lymphoblastic leukemia and predicts clinical outcome. Haematologica 2011; 96(7): 980-986. 
235. Rakhmanov M, Sic H, Kienzler AK, Fischer B, Rizzi M, Seidl M, et al. High levels of SOX5 decrease proliferative capacity of human B cells, but permit plasmablast differentiation. PLoS One 2014; 9(6): e100328.

236. Edwards SK, Desai A, Liu Y, Moore CR, Xie P. Expression and function of a novel isoform of Sox 5 in malignant B cells. Leuk Res 2014; 38(3): 393-401.

237. Bridges LC, Tani PH, Hanson KR, Roberts CM, Judkins MB, Bowditch RD. The lymphocyte metalloprotease MDC-L (ADAM 28) is a ligand for the integrin alpha4beta1. J Biol Chem 2002; 277(5): 3784-3792.

238. Meyer C, Schneider B, Reichel M, Angermueller S, Strehl S, Schnittger S, et al. Diagnostic tool for the identification of MLL rearrangements including unknown partner genes. Proc Natl Acad Sci U S A 2005; 102(2): 449-454.

239. Moreau K, Dizin E, Ray H, Luquain C, Lefai E, Foufelle F, et al. BRCA1 affects lipid synthesis through its interaction with acetyl-CoA carboxylase. J Biol Chem 2006; 281(6): 3172-3181.

240. Abramson HN. The lipogenesis pathway as a cancer target. J Med Chem 2011; 54(16): 5615-5638.

241. Magnard C, Bachelier R, Vincent A, Jaquinod M, Kieffer S, Lenoir GM, et al. BRCA1 interacts with acetyl-CoA carboxylase through its tandem of BRCT domains. Oncogene 2002; 21(44): 6729-6739.

242. Sinilnikova OM, Ginolhac SM, Magnard C, Leone M, Anczukow O, Hughes D, et al. Acetyl-CoA carboxylase alpha gene and breast cancer susceptibility. Carcinogenesis 2004; 25(12): 2417-2424.

243. Meyer C, Kowarz E, Hofmann J, Renneville A, Zuna J, Trka J, et al. New insights to the MLL recombinome of acute leukemias. Leukemia 2009; 23(8): 1490-1499.

244. Slany RK. The molecular biology of mixed lineage leukemia. Haematologica 2009; 94(7): 984-993.

245. Watanabe N, Wachi S, Fujita T. Identification and characterization of BCL-3binding protein: implications for transcription and DNA repair or recombination. J Biol Chem 2003; 278(28): 26102-26110.

246. Robinson HM, Taylor KE, Jalali GR, Cheung KL, Harrison CJ, Moorman AV. $\mathrm{t}(14 ; 19)(\mathrm{q} 32 ; \mathrm{q} 13)$ : a recurrent translocation in B-cell precursor acute lymphoblastic leukemia. Genes Chromosomes Cancer 2004; 39(1): 88-92. 
247. McKeithan TW, Takimoto GS, Ohno H, Bjorling VS, Morgan R, Hecht BK, et al. BCL3 rearrangements and $t(14 ; 19)$ in chronic lymphocytic leukemia and other Bcell malignancies: a molecular and cytogenetic study. Genes Chromosomes Cancer 1997; 20(1): 64-72.

248. Ross TS, Bernard OA, Berger R, Gilliland DG. Fusion of Huntingtin interacting protein 1 to platelet-derived growth factor beta receptor (PDGFbetaR) in chronic myelomonocytic leukemia with $\mathrm{t}(5 ; 7)(\mathrm{q} 33 ; \mathrm{q} 11.2)$. Blood 1998; 91(12): 44194426.

249. Velloso ER, Michaux L, Ferrant A, Hernandez JM, Meeus P, Dierlamm J, et al. Deletions of the long arm of chromosome 7 in myeloid disorders: loss of band 7q32 implies worst prognosis. Br J Haematol 1996; 92(3): 574-581.

250. Salemi M, Barone C, Romano C, Zolezzi F, Scavuzzo C, Salluzzo R, et al. Gene expression profiling and qRT-PCR expression of RRP1B, PCNT, KIF21A and ADRB2 in leucocytes of Down's syndrome subjects. J Genet 2012; 91(1): e18-23.

251. Salemi M, Barone C, Romano C, Ridolfo F, Scavuzzo C, Cantarella RA, et al. KIF21A mRNA expression in patients with Down syndrome. Neurol Sci 2013; 34(4): 569-571.

252. Levy AS, Sather HN, Steinherz PG, Sowers R, La M, Moscow JA, et al. Reduced folate carrier and dihydrofolate reductase expression in acute lymphocytic leukemia may predict outcome: a Children's Cancer Group Study. J Pediatr Hematol Oncol 2003; 25(9): 688-695.

253. Matheson EC, Hogarth LA, Case MC, Irving JA, Hall AG. DHFR and MSH3 coamplification in childhood acute lymphoblastic leukaemia, in vitro and in vivo. Carcinogenesis 2007; 28(6): 1341-1346.

254. Kraft P, Zeggini E, Ioannidis JP. Replication in genome-wide association studies. Stat Sci 2009; 24(4): 561-573.

255. Knight T, Irving JA. Ras/Raf/MEK/ERK Pathway Activation in Childhood Acute Lymphoblastic Leukemia and Its Therapeutic Targeting. Front Oncol 2014; 4: 160.

256. Aster JC, Pear WS, Blacklow SC. Notch signaling in leukemia. Annu Rev Pathol 2008; 3: 587-613.

257. Lin S, Tian L, Shen H, Gu Y, Li JL, Chen Z, et al. DDX5 is a positive regulator of oncogenic NOTCH1 signaling in T cell acute lymphoblastic leukemia. Oncogene 2013; 32(40): 4845-4853. 
258. Brimer N, Lyons C, Wallberg AE, Vande Pol SB. Cutaneous papillomavirus E6 oncoproteins associate with MAML1 to repress transactivation and $\mathrm{NOTCH}$ signaling. Oncogene 2012; 31(43): 4639-4646.

259. Zhao Y, Katzman RB, Delmolino LM, Bhat I, Zhang Y, Gurumurthy CB, et al. The notch regulator MAML1 interacts with p53 and functions as a coactivator. $J$ Biol Chem 2007; 282(16): 11969-11981.

260. Akino K, Toyota M, Suzuki H, Mita H, Sasaki Y, Ohe-Toyota M, et al. The Ras effector RASSF2 is a novel tumor-suppressor gene in human colorectal cancer. Gastroenterology 2005; 129(1): 156-169.

261. Vos MD, Ellis CA, Elam C, Ulku AS, Taylor BJ, Clark GJ. RASSF2 is a novel K-Ras-specific effector and potential tumor suppressor. J Biol Chem 2003; 278(30): 28045-28051.

262. Case M, Matheson E, Minto L, Hassan R, Harrison CJ, Bown N, et al. Mutation of genes affecting the RAS pathway is common in childhood acute lymphoblastic leukemia. Cancer Res 2008; 68(16): 6803-6809.

263. Perentesis JP, Bhatia S, Boyle E, Shao Y, Shu XO, Steinbuch M, et al. RAS oncogene mutations and outcome of therapy for childhood acute lymphoblastic leukemia. Leukemia 2004; 18(4): 685-692.

264. Llamazares M, Obaya AJ, Moncada-Pazos A, Heljasvaara R, Espada J, LopezOtin C, et al. The ADAMTS12 metalloproteinase exhibits anti-tumorigenic properties through modulation of the Ras-dependent ERK signalling pathway. $J$ Cell Sci 2007; 120(20): 3544-3552.

265. Matsushima-Nishiwaki R, Kumada T, Nagasawa T, Suzuki M, Yasuda E, Okuda $\mathrm{S}$, et al. Direct association of heat shock protein 20 (HSPB6) with phosphoinositide 3-kinase (PI3K) in human hepatocellular carcinoma: regulation of the PI3K activity. PLoS One 2013; 8(11): e78440.

266. Jackson RS, 2nd, Cho YJ, Stein S, Liang P. CYFIP2, a direct p53 target, is leptomycin-B sensitive. Cell Cycle 2007; 6(1): 95-103.

267. Yao H, Li P, Venters BJ, Zheng S, Thompson PR, Pugh BF, et al. Histone Arg modifications and p53 regulate the expression of OKL38, a mediator of apoptosis. J Biol Chem 2008; 283(29): 20060-20068.

268. Tian C, Xing G, Xie P, Lu K, Nie J, Wang J, et al. KRAB-type zinc-finger protein Apak specifically regulates p53-dependent apoptosis. Nat Cell Biol 2009; 11(5): 580-591. 
269. Wu M, Xu LG, Su T, Tian Y, Zhai Z, Shu HB. AMID is a p53-inducible gene downregulated in tumors. Oncogene 2004; 23(40): 6815-6819.

270. Ohiro Y, Garkavtsev I, Kobayashi S, Sreekumar KR, Nantz R, Higashikubo BT, et al. A novel p53-inducible apoptogenic gene, PRG3, encodes a homologue of the apoptosis-inducing factor (AIF). FEBS Lett 2002; 524(1-3): 163-171.

271. Kudo T, Ikeda M, Nishikawa M, Yang Z, Ohno K, Nakagawa K, et al. The RASSF3 candidate tumor suppressor induces apoptosis and G1-S cell-cycle arrest via p53. Cancer Res 2012; 72(11): 2901-2911.

272. Levine AJ, Hu W, Feng Z. The P53 pathway: what questions remain to be explored? Cell Death Differ 2006; 13(6): 1027-1036.

273. Langlois B, Perrot G, Schneider C, Henriet P, Emonard H, Martiny L, et al. LRP1 promotes cancer cell invasion by supporting ERK and inhibiting JNK signaling pathways. PLoS One 2010; 5(7): e11584.

274. Hindorff LA, Sethupathy P, Junkins HA, Ramos EM, Mehta JP, Collins FS, et al. Potential etiologic and functional implications of genome-wide association loci for human diseases and traits. Proc Natl Acad Sci U S A 2009; 106(23): $9362-$ 9367.

275. Witte JS. Genome-wide association studies and beyond. Annu Rev Public Health 2010; 31: 9-20

276. Hunter DJ. Gene-environment interactions in human diseases. Nat Rev Genet 2005; 6(4): 287-298.

277. Davis AP, Grondin CJ, Lennon-Hopkins K, Saraceni-Richards C, Sciaky D, King BL, et al. The Comparative Toxicogenomics Database's 10th year anniversary: update 2015. Nucleic Acids Res 2015; 43: D914-920.

278. Vinceti M, Rothman KJ, Crespi CM, Sterni A, Cherubini A, Guerra L, et al. Leukemia risk in children exposed to benzene and PM10 from vehicular traffic: a case-control study in an Italian population. Eur J Epidemiol 2012; 27(10): 781790 .

279. Perera FP. Environment and cancer: who are susceptible? Science 1997; 278(5340): 1068-1073.

280. Buffler PA, Kwan ML, Reynolds P, Urayama KY. Environmental and genetic risk factors for childhood leukemia: appraising the evidence. Cancer Invest 2005; 23(1): $60-75$. 
281. Myers RA, Scott NM, Gauderman WJ, Qiu W, Mathias RA, Romieu I, et al. Genome-wide interaction studies reveal sex-specific asthma risk alleles. Hum Mol Genet 2014; 23(19): 5251-5259.

282. Yao C, Joehanes R, Johnson AD, Huan T, Esko T, Ying S, et al. Sex- and ageinteracting eQTLs in human complex diseases. Hum Mol Genet 2014; 23(7): 1947-1956.

283. Van der Meulen J, Sanghvi V, Mavrakis K, Durinck K, Fang F, Matthijssens F, et al. The H3K27me3 demethylase UTX is a gender-specific tumor suppressor in Tcell acute lymphoblastic leukemia. Blood 2015; 125(1): 13-21.

284. Mez JB, Cole JW, Howard TD, Macclellan LR, Stine OC, O'Connell JR, et al. Evaluation of self-reported ethnicity in a case-control population: the stroke prevention in young women study. BMC Res Notes 2009; 2: 260.

285. Galichon P, Mesnard L, Hertig A, Stengel B, Rondeau E. Unrecognized sequence homologies may confound genome-wide association studies. Nucleic Acids Res 2012; 40(11): 4774-4782. 
APPENDIX 
Appendix Table 1: Genetic risk markers for childhood acute lymphoblastic leukemia in previous genome-wide association studies

\begin{tabular}{|c|c|c|c|c|c|}
\hline SNP & Gene & Gene function & OR (95\% CI) & P value & Ref \\
\hline \multirow{2}{*}{ rs 10821936} & \multirow{2}{*}{ ARID5B } & \multirow{2}{*}{$\begin{array}{l}\text { differentiation of } \mathrm{B} \\
\text { lymphocyte progenitor }\end{array}$} & $1.91(1.60-2.20)$ & $1.4 \times 10^{-15}$ & 51 \\
\hline & & & $1.86(1.71-2.03)$ & $5.90 \times 10^{-46}$ & 53 \\
\hline rs10994982 & ARID5B & $\begin{array}{c}\text { differentiation of } \mathrm{B} \\
\text { lymphocyte progenitor }\end{array}$ & $1.61(1.30-1.90)$ & $5.70 \times 10^{-9}$ & 51 \\
\hline \multirow{2}{*}{ rs7073837 } & \multirow{2}{*}{ ARID5B } & \multirow{2}{*}{$\begin{array}{l}\text { differentiation of } \mathrm{B} \\
\text { lymphocyte progenitor }\end{array}$} & $1.58(1.35-1.89)$ & $4.66 \times 10^{-16}$ & 52 \\
\hline & & & $1.64(1.40-1.92)$ & $1.0 \times 10^{-9}$ & 49 \\
\hline \multirow{2}{*}{ rs 10740055} & \multirow{2}{*}{ ARID5B } & \multirow{2}{*}{$\begin{array}{l}\text { differentiation of } \mathrm{B} \\
\text { lymphocyte progenitor }\end{array}$} & $1.53(1.41-1.64)$ & $5.35 \times 10^{-14}$ & 52 \\
\hline & & & $1.75(1.49-2.06)$ & $1.8 \times 10^{-11}$ & 49 \\
\hline \multirow{2}{*}{ rs7089424 } & \multirow{2}{*}{ ARID5B } & \multirow{2}{*}{$\begin{array}{l}\text { differentiation of B } \\
\text { lymphocyte progenitor }\end{array}$} & $1.65(1.54-1.76)$ & $6.69 \times 10^{-19}$ & 52 \\
\hline & & & $1.83(1.55-2.15)$ & $6.13 \times 10^{-13}$ & 49 \\
\hline rs11978267 & $I K Z F 1$ & $\begin{array}{c}\text { regulator of lymphocyte } \\
\text { differentiation }\end{array}$ & $1.69(1.40-1.90)$ & $8.8 \times 10^{-11}$ & 51 \\
\hline \multirow{2}{*}{ rs4132601 } & \multirow{2}{*}{$I K Z F 1$} & \multirow{2}{*}{$\begin{array}{l}\text { regulator of lymphocyte } \\
\text { differentiation }\end{array}$} & $1.69(1.58-1.81)$ & $1.20 \times 10^{-19}$ & 52 \\
\hline & & & $1.59(1.34-1.89)$ & $1.2 \times 10^{-7}$ & 49 \\
\hline rs6964969 & $I K Z F 1$ & $\begin{array}{c}\text { regulator of lymphocyte } \\
\text { differentiation }\end{array}$ & $1.59(1.45-1.74)$ & $1.59 \times 10^{-29}$ & 53 \\
\hline rs2239633 & CEBPE & $\begin{array}{c}\text { functional maturation of } \\
\text { granulocyte progenitor } \\
\text { cells }\end{array}$ & $1.34(1.22-1.45)$ & $2.88 \times 10^{-7}$ & 52 \\
\hline rs4982731 & CEBPE & $\begin{array}{c}\text { functional maturation of } \\
\text { granulocyte progenitor } \\
\text { cells }\end{array}$ & $1.36(1.24-1.48)$ & $8.97 \times 10^{-12}$ & 53 \\
\hline rs2089222 & KRT85 & hair and nail formation & $2.26(1.60-3.00)$ & $8.40 \times 10^{-8}$ & 51 \\
\hline rs11155133 & $6 q 24$ & NA & $3.62(2.10-6.00)$ & $3.0 \times 10^{-7}$ & 51 \\
\hline rs2191566 & ZNF230 & NA & $1.52(1.20-1.70)$ & $3.5 \times 10^{-7}$ & 51 \\
\hline rs6509133 & ZNF230 & NA & $1.48(1.20-1.70)$ & $1.4 \times 10^{-6}$ & 51 \\
\hline rs7554607 & RYR2 & calcium channel & $1.49(1.20-1.70)$ & $1.6 \times 10^{-6}$ & 51 \\
\hline rs12621643 & KCNE4 & $\begin{array}{c}\text { voltage-gated } \\
\text { potassium channel } \\
\text { activity } \\
\end{array}$ & $1.48(1.20-1.70)$ & $2.8 \times 10^{-6}$ & 51 \\
\hline rs 10873876 & ST6GALNAC3 & NA & $1.55(1.20-1.80)$ & $4 \times 10^{-6}$ & 51 \\
\hline rs9290663 & KCNMB2 & $\begin{array}{c}\text { voltage-gated } \\
\text { potassium channel } \\
\text { activity }\end{array}$ & $1.58(1.20-1.90)$ & $6 \times 10^{-6}$ & 51 \\
\hline rs6428370 & $1 \mathrm{q} 31$ & NA & $1.43(1.20-1.60)$ & $7 \times 10^{-6}$ & 51 \\
\hline rs1881797 & OR2C3 & $\begin{array}{l}\text { G protein coupled } \\
\text { receptor activity }\end{array}$ & $1.52(1.20-1.80)$ & $7 \times 10^{-6}$ & 51 \\
\hline
\end{tabular}


Appendix Table 1 continued....

\begin{tabular}{|c|c|c|c|c|c|}
\hline SNP & Gene & Gene function & OR $(95 \%$ CI $)$ & P value & Ref \\
\hline rs10849033 & C12orf5 & $\begin{array}{l}\text { TP53-induced } \\
\text { glycolysis and } \\
\text { apoptosis }\end{array}$ & $2.55(1.60-3.80)$ & $9 \times 10^{-6}$ & 51 \\
\hline rs1879352 & 18 p11.32 & NA & $1.53(1.20-1.80)$ & $9 \times 10^{-6}$ & 51 \\
\hline rs563507 & PARD3 & $\begin{array}{c}\text { protein phosphatase } \\
\text { binding }\end{array}$ & $2.00(1.40-2.70)$ & $9 \times 10^{-6}$ & 51 \\
\hline rs3779084 & $D D C$ & $\begin{array}{l}\text { amino acid binding and } \\
\text { protein domain specific } \\
\text { binding }\end{array}$ & $1.44(1.32-1.56)$ & $8.81 \times 10^{-9}$ & 52 \\
\hline rs 880028 & $D D C$ & $\begin{array}{l}\text { amino acid binding and } \\
\text { protein domain specific } \\
\text { binding }\end{array}$ & $1.43(1.30-1.56)$ & $1.26 \times 10^{-7}$ & 52 \\
\hline rs 7809758 & $D D C$ & $\begin{array}{l}\text { amino acid binding and } \\
\text { protein domain specific } \\
\text { binding }\end{array}$ & $1.44(1.32-1.54)$ & $2.41 \times 10^{-10}$ & 52 \\
\hline rs2242041 & $D D C$ & $\begin{array}{l}\text { amino acid binding and } \\
\text { protein domain specific } \\
\text { binding }\end{array}$ & $1.72(1.30-2.10)$ & $9.9 \times 10^{-7}$ & 51 \\
\hline rs2167364 & $D D C$ & $\begin{array}{l}\text { amino acid binding and } \\
\text { protein domain specific } \\
\text { binding }\end{array}$ & $1.46(1.20-1.70)$ & $2.8 \times 10^{-6}$ & 51 \\
\hline rs930372 & ANKRD44 & $\begin{array}{c}\text { recognition of } \\
\text { phosphoprotein } \\
\text { substrate }\end{array}$ & $2.37(1.65-3.41)$ & $3.1 \times 10^{-6}$ & 49 \\
\hline rs1945213 & OR8U8 & $\begin{array}{l}\text { G protein coupled } \\
\text { receptor activity }\end{array}$ & $1.30(1.19-1.43)$ & $3 \times 10^{-8}$ & 54 \\
\hline rs17505102 & TP63 & $\begin{array}{c}\text { sequence specific DNA } \\
\text { binding transcriptional } \\
\text { activator or repressor }\end{array}$ & $1.47(1.28-1.67)$ & $2 \times 10-8$ & 54 \\
\hline rs7156960 & GPATCH2L & NA & $1.22(1.12-1.33)$ & $3 \times 10^{-6}$ & 54 \\
\hline rs7738636 & $6 q 14$ & NA & $1.27(1.15-1.41)$ & $6 \times 10^{-6}$ & 54 \\
\hline rs282708 & $4 q 13.1$ & NA & $1.23(1.12-1.35)$ & $8 \times 10^{-6}$ & 54 \\
\hline rs4748793 & COMMD3 & $\begin{array}{c}\text { may down-regulate } \\
\text { activation of NF-kappa- } \\
\text { B }\end{array}$ & $1.40(1.26-1.57)$ & $8.40 \times 10^{-9}$ & 53 \\
\hline rs3776932 & MAN2A1 & $\begin{array}{c}\text { biosynthesis of } \\
\text { complex N-glycans }\end{array}$ & $0.23(0.10-0.50)$ & 0.0006 & 50 \\
\hline rs6140264 & HAO1 & $\begin{array}{c}\text { has 2-hydroxyacid } \\
\text { oxidase activity }\end{array}$ & $4.33(1.8-10.20)$ & 0.0008 & 50 \\
\hline rs12105972 & GCFC2 & $\begin{array}{l}\text { factor that represses } \\
\text { transcription, bind to } \\
\text { GC-rich sequences }\end{array}$ & $0.30(0.10-0.60)$ & 0.0015 & 50 \\
\hline rs17837497 & TBXAS1 & $\begin{array}{c}\text { electron carrier activity } \\
\text { and heme binding }\end{array}$ & $2.34(1.65-3.31)$ & $1.89 \times 10^{-6}$ & 219 \\
\hline rs1496766 & MAGI2 & $\begin{array}{c}\text { enhances the ability of } \\
P T E N \text { to suppress } \\
\text { AKT1 activation }\end{array}$ & $2.84(1.81-4.44)$ & $5.45 \times 10^{-6}$ & 219 \\
\hline
\end{tabular}


Appendix Table 1 continued....

\begin{tabular}{|c|c|c|c|c|c|}
\hline SNP & Gene & Gene function & OR (95\% CI) & P value & Ref \\
\hline rs17079534 & MYRIP & melanosome transport & $4.07(2.04-6.87)$ & $1.65 \times 10^{-7}$ & 219 \\
\hline rs10170236 & LOC642340 & NA & $1.45(1.24-1.69)$ & $3.92 \times 10^{-6}$ & 219 \\
\hline rs7578361 & $2 \mathrm{q} 23$ & NA & $1.40(1.21-1.63)$ & $8.40 \times 10^{-6}$ & 219 \\
\hline rs7142143 & PYGL & $\begin{array}{c}\text { pyridoxal phosphate } \\
\text { binding and protein } \\
\text { homodimerization } \\
\text { activity }\end{array}$ & $3.61(2.34-5.57)$ & $6.72 \times 10^{-9}$ & 219 \\
\hline rs6683977 & PDE4B & ion channel binding & $1.41(1.22-1.64)$ & $5.13 \times 10^{-6}$ & 219 \\
\hline rs546784 & PDE4B & ion channel binding & $1.40(1.20-1.62)$ & $1.17 \times 10^{-5}$ & 219 \\
\hline
\end{tabular}

$\mathrm{SNP}=$ single nucleotide polymorphism; $\mathrm{OR}=$ odds ratio; $\mathrm{CI}=$ confidence interval; Ref $=$ reference 
Appendix Table 2: Top one hundred SNPs associated with childhood ALL risk with gender-specificity, results based on the lowest $P$ values

\begin{tabular}{|c|c|c|c|c|c|c|c|c|c|}
\hline \multirow[b]{2}{*}{ SNP } & \multirow[b]{2}{*}{ Gene/region } & \multirow[b]{2}{*}{$\mathrm{Chr}^{\dagger}$} & \multirow[b]{2}{*}{$\begin{array}{l}\text { Minor } \\
\text { allele }\end{array}$} & \multirow[b]{2}{*}{ Feature } & \multicolumn{2}{|c|}{$\begin{array}{l}\text { Minor allele } \\
\text { frequency }\end{array}$} & \multirow[b]{2}{*}{$\mathrm{OR}_{\text {allele }}(95 \% \mathrm{CI})^{*}$} & \multirow[b]{2}{*}{ P value** } & \multirow[b]{2}{*}{$\begin{array}{c}\text { P value } \\
\text { (permutation) }^{\dagger \dagger}\end{array}$} \\
\hline & & & & & Males & Females & & & \\
\hline rs 4813720 & RASSF2 & 20 & $\mathrm{~A}$ & 5upstream & 0.23 & 0.47 & $0.29(0.17-0.48)$ & $1.90 \times 10^{-06}$ & $1.0 \times 10^{-4}$ \\
\hline rs231237 & HSPB6 & 19 & A & 5upstream & 0.42 & 0.21 & $3.00(1.86-4.85)$ & $7.15 \times 10^{-06}$ & $1.0 \times 10^{-4}$ \\
\hline rs7323018 & $13 \mathrm{q} 14.1$ & 13 & G & intergenic & 0.17 & 0.36 & $0.31(0.18-0.52)$ & $8.40 \times 10^{-06}$ & $1.0 \times 10^{-4}$ \\
\hline rs 17027254 & $2 \mathrm{p} 22$ & 2 & $\mathrm{~T}$ & intergenic & 0.19 & 0.39 & $0.37(0.23-0.58)$ & $1.86 \times 10^{-05}$ & $1.0 \times 10^{-4}$ \\
\hline rs7912381 & $10 \mathrm{q} 26.3$ & 10 & G & intergenic & 0.39 & 0.21 & $2.78(1.71-4.51)$ & $3.68 \times 10^{-05}$ & $1.0 \times 10^{-4}$ \\
\hline rs11687208 & $2 \mathrm{p} 22$ & 2 & $\mathrm{C}$ & intergenic & 0.21 & 0.4 & $0.40(0.26-0.62)$ & $4.81 \times 10^{-05}$ & $1.0 \times 10^{-4}$ \\
\hline rs798292 & MAGI2 & 7 & G & intronic & 0.16 & 0.35 & $0.38(0.24-0.61)$ & $4.88 \times 10^{-05}$ & $1.5 \times 10^{-4}$ \\
\hline rs13107783 & $4 p 15.3$ & 4 & $\mathrm{C}$ & intergenic & 0.32 & 0.52 & $0.40(0.26-0.62)$ & $5.16 \times 10^{-05}$ & $2.0 \times 10^{-4}$ \\
\hline rs4470624 & $4 p 15.3$ & 4 & $\mathrm{~T}$ & intergenic & 0.32 & 0.52 & $0.40(0.25-0.62)$ & $5.16 \times 10^{-05}$ & $2.0 \times 10^{-4}$ \\
\hline rs 1849374 & $12 \mathrm{p} 11.2$ & 12 & A & intergenic & 0.44 & 0.25 & $2.48(1.58-3.88)$ & $7.35 \times 10^{-05}$ & $2.0 \times 10^{-4}$ \\
\hline rs 12948120 & $\overline{A C A C A}$ & 17 & $\mathrm{C}$ & intronic & 0.29 & 0.49 & $0.44(0.29-0.66)$ & $7.46 \times 10^{-05}$ & $3.0 \times 10^{-4}$ \\
\hline rs6904762 & $6 \mathrm{q} 23$ & 6 & G & intergenic & 0.27 & 0.46 & $0.41(0.26-0.64)$ & $1.00 \times 10^{-05}$ & $1.0 \times 10^{-4}$ \\
\hline rs506389 & $8 \mathrm{q} 22.3$ & 8 & A & intergenic & 0.28 & 0.11 & $2.99(1.74-5.15)$ & $7.98 \times 10^{-05}$ & $2.0 \times 10^{-4}$ \\
\hline rs6732320 & $2 \mathrm{p} 22$ & 2 & $\mathrm{~T}$ & intergenic & 0.2 & 0.38 & $0.41(0.26-0.64)$ & $8.08 \times 10^{-05}$ & $3.0 \times 10^{-4}$ \\
\hline rs8081866 & ACACA & 17 & $\mathrm{~T}$ & intronic & 0.28 & 0.48 & $0.45(0.30-0.67)$ & $8.64 \times 10^{-05}$ & $3.0 \times 10^{-4}$ \\
\hline rs349714 & $3 \mathrm{p} 25$ & 3 & $\bar{G}$ & intergenic & 0.44 & 0.26 & $2.43(1.56-3.80)$ & $8.76 \times 10^{-05}$ & $2.0 \times 10^{-4}$ \\
\hline rs7583193 & $2 \mathrm{p} 22$ & 2 & $\bar{G}$ & intergenic & 0.19 & 0.38 & $0.41(0.26-0.64)$ & $9.09 \times 10^{-05}$ & $1.0 \times 10^{-4}$ \\
\hline rs206457 & $18 \mathrm{p} 11.2$ & 18 & A & intergenic & 0.59 & 0.38 & $2.24(1.49-3.35)$ & $9.32 \times 10^{-04}$ & $1.0 \times 10^{-4}$ \\
\hline rs7723568 & $5 \mathrm{p} 15.3$ & 5 & A & intergenic & 0.35 & 0.18 & $2.66(1.62-4.36)$ & $1.10 \times 10^{-04}$ & $2.0 \times 10^{-4}$ \\
\hline rs 2542660 & ACACA & 17 & $\mathrm{C}$ & intronic & 0.26 & 0.46 & $0.45(0.30-0.68)$ & $1.16 \times 10^{-04}$ & $3.0 \times 10^{-4}$ \\
\hline
\end{tabular}


Appendix Table 2 continued....

\begin{tabular}{|c|c|c|c|c|c|c|c|c|c|}
\hline \multirow[b]{2}{*}{ SNP } & \multirow[b]{2}{*}{ Gene/region } & \multirow[b]{2}{*}{$\mathrm{Chr}^{\dagger}$} & \multirow[b]{2}{*}{$\begin{array}{l}\text { Minor } \\
\text { allele }\end{array}$} & \multirow[b]{2}{*}{ Feature } & \multicolumn{2}{|c|}{$\begin{array}{l}\text { Minor allele } \\
\text { frequency }\end{array}$} & \multirow[b]{2}{*}{$\mathrm{OR}_{\text {allele }}(95 \% \mathrm{CI})^{*}$} & \multirow[b]{2}{*}{$P$ value** } & \multirow[b]{2}{*}{$\begin{array}{c}\text { P value } \\
\text { (permutation) }^{\dagger \dagger}\end{array}$} \\
\hline & & & & & Males & Females & & & \\
\hline rs1899286 & $2 \mathrm{p} 22$ & 2 & $\mathrm{~A}$ & intergenic & 0.21 & 0.39 & $0.42(0.27-0.66)$ & $1.19 \times 10^{-04}$ & $2.0 \times 10^{-4}$ \\
\hline rs9563035 & $13 q 14$ & 13 & G & intergenic & 0.5 & 0.31 & $2.30(1.50-3.52)$ & $1.24 \times 10^{-04}$ & $1.0 \times 10^{-4}$ \\
\hline rs 2542653 & $\overline{A C A C A}$ & 17 & $\mathrm{C}$ & intronic & 0.26 & 0.46 & $0.46(0.31-0.68)$ & $1.32 \times 10^{-04}$ & $2.0 \times 10^{-4}$ \\
\hline rs6490437 & $13 q 12$ & 13 & G & intergenic & 0.41 & 0.6 & $0.44(0.29-0.67)$ & $1.34 \times 10^{-04}$ & $2.0 \times 10^{-4}$ \\
\hline rs4794031 & $17 q 21$ & 17 & $\mathrm{C}$ & intergenic & 0.43 & 0.24 & $2.32(1.50-3.58)$ & $1.42 \times 10^{-04}$ & $3.0 \times 10^{-4}$ \\
\hline rs 2544371 & $2 \mathrm{q} 31$ & 2 & G & intergenic & 0.37 & 0.57 & $0.45(0.30-0.68)$ & $1.52 \times 10^{-04}$ & $1.0 \times 10^{-4}$ \\
\hline rs 1372166 & $2 \mathrm{p} 22$ & 2 & $\mathrm{C}$ & intergenic & 0.13 & 0.28 & $0.36(0.21-0.61)$ & $1.83 \times 10^{-04}$ & $2.0 \times 10^{-4}$ \\
\hline rs7294845 & SOX5 & 12 & $\mathrm{~T}$ & intronic & 0.20 & 0.37 & $0.41(0.26-0.65)$ & $1.59 \times 10^{-04}$ & $1.0 \times 10^{-4}$ \\
\hline rs12219278 & $10 \mathrm{q} 26$ & 10 & $\mathrm{~T}$ & intergenic & 0.29 & 0.14 & $2.74(1.62-4.64)$ & $1.79 \times 10^{-04}$ & $2.0 \times 10^{-4}$ \\
\hline rs4942264 & $13 q 13$ & 13 & $\mathrm{~T}$ & intergenic & 0.12 & 0.26 & $0.36(0.21-0.62)$ & $2.04 \times 10^{-04}$ & $2.5 \times 10^{-4}$ \\
\hline rs11708505 & $3 p 13$ & 3 & A & intergenic & 0.41 & 0.6 & $0.47(0.31-0.70)$ & $2.11 \times 10^{-04}$ & $2.0 \times 10^{-4}$ \\
\hline rs6540610 & HHAT & 1 & A & intronic & 0.18 & 0.39 & $0.39(0.24-0.65)$ & $2.18 \times 10^{-04}$ & $4.0 \times 10^{-4}$ \\
\hline rs2764928 & $1 \mathrm{p} 32$ & 1 & A & intergenic & 0.12 & 0.26 & $0.36(0.21-0.62)$ & $2.43 \times 10^{-04}$ & $2.0 \times 10^{-4}$ \\
\hline rs 1550017 & $4 q 32$ & 4 & A & intergenic & 0.17 & 0.05 & $4.23(1.96-9.14)$ & $2.45 \times 10^{-04}$ & $1.0 \times 10^{-4}$ \\
\hline rs12054636 & $4 \mathrm{q} 32$ & 4 & A & intergenic & 0.17 & 0.05 & $4.23(1.96-9.14)$ & $2.45 \times 10^{-04}$ & $1.0 \times 10^{-4}$ \\
\hline rs2764933 & $1 \mathrm{p} 32$ & 1 & $\mathrm{~A}$ & intergenic & 0.32 & 0.51 & $0.45(0.30-0.69)$ & $2.48 \times 10^{-04}$ & $2.0 \times 10^{-4}$ \\
\hline rs468771 & GRIK1 & 21 & $\mathrm{~T}$ & intronic & 0.33 & 0.17 & $2.48(1.52-4.02)$ & $2.54 \times 10^{-04}$ & $3.0 \times 10^{-4}$ \\
\hline rs864674 & $5 \mathrm{p} 13$ & 5 & $\mathrm{C}$ & intergenic & 0.55 & 0.38 & $2.27(1.46-3.53)$ & $2.65 \times 10^{-04}$ & $1.0 \times 10^{-4}$ \\
\hline rs8106959 & KMT2B & 19 & $\bar{G}$ & intronic & 0.25 & 0.11 & $2.87(1.63-5.07)$ & $2.66 \times 10^{-04}$ & $3.0 \times 10^{-4}$ \\
\hline rs9561489 & GPC6 & 13 & A & intronic & 0.40 & 0.57 & $0.45(0.29-0.70)$ & $2.86 \times 10^{-04}$ & $4.0 \times 10^{-4}$ \\
\hline rs4934956 & 10p11.2 & 10 & $\mathrm{~T}$ & intergenic & 0.56 & 0.38 & $2.20(1.44-3.37)$ & $2.91 \times 10^{-04}$ & $3.0 \times 10^{-4}$ \\
\hline
\end{tabular}


Appendix Table 2 continued....

\begin{tabular}{|c|c|c|c|c|c|c|c|c|c|}
\hline \multirow[b]{2}{*}{ SNP } & \multirow[b]{2}{*}{ Gene/region } & \multirow[b]{2}{*}{$\mathrm{Chr}^{\dagger}$} & \multirow[b]{2}{*}{$\begin{array}{l}\text { Minor } \\
\text { allele }\end{array}$} & \multirow[b]{2}{*}{ Feature } & \multicolumn{2}{|c|}{$\begin{array}{l}\text { Minor allele } \\
\text { frequency }\end{array}$} & \multirow[b]{2}{*}{$\mathrm{OR}_{\text {allele }}(95 \% \mathrm{CI})^{*}$} & \multirow[b]{2}{*}{ P value** } & \multirow[b]{2}{*}{$\begin{array}{c}\text { P value } \\
\text { (permutation) }^{\dagger \dagger}\end{array}$} \\
\hline & & & & & Males & Females & & & \\
\hline rs9897342 & $17 \mathrm{q} 11.1$ & 17 & $\mathrm{~A}$ & intergenic & 0.43 & 0.26 & $2.21(1.44-3.40)$ & $3.03 \times 10^{-04}$ & $5.0 \times 10^{-4}$ \\
\hline rs 756134 & $1 \mathrm{p} 13$ & 1 & A & intergenic & 0.53 & 0.35 & $2.10(1.40-3.15)$ & $3.11 \times 10^{-04}$ & $4.0 \times 10^{-4}$ \\
\hline rs4926465 & KIF28P & 1 & G & 5upstream & 0.49 & 0.44 & $0.43(0.27-0.68)$ & $3.14 \times 10^{-04}$ & $3.0 \times 10^{-4}$ \\
\hline rs552976 & $A B C B 11$ & 2 & $\mathrm{~T}$ & intronic & 0.17 & 0.53 & $0.40(0.24-0.66)$ & $3.15 \times 10^{-04}$ & $1.0 \times 10^{-4}$ \\
\hline rs563694 & $2 q 31$ & 2 & G & intergenic & 0.16 & 0.54 & $0.38(0.23-0.65)$ & $3.20 \times 10^{-04}$ & $3.0 \times 10^{-4}$ \\
\hline rs 1529186 & $3 q 13.1$ & 3 & A & intergenic & 0.14 & 0.29 & $0.40(0.24-0.66)$ & $3.47 \times 10^{-04}$ & $2.0 \times 10^{-4}$ \\
\hline rs11049786 & $12 \mathrm{p} 11.2$ & 12 & G & intergenic & 0.44 & 0.27 & $2.24(1.44-3.50)$ & $3.50 \times 10^{-04}$ & $4.0 \times 10^{-4}$ \\
\hline rs9510793 & TNFRSF19 & 13 & $\mathrm{C}$ & intronic & 0.35 & 0.20 & $2.40(1.49-3.88)$ & $3.51 \times 10^{-04}$ & $3.0 \times 10^{-4}$ \\
\hline rs61748746 & N4BP2 & 4 & A & coding & 0.02 & 0.10 & $0.10(0.02-0.36)$ & $3.64 \times 10^{-04}$ & $1.5 \times 10^{-4}$ \\
\hline rs2874641 & SHISA6 & 17 & G & intronic & 0.19 & 0.35 & $0.43(0.27-0.69)$ & $3.64 \times 10^{-04}$ & $2.0 \times 10^{-4}$ \\
\hline rs560887 & G6PC2 & 2 & A & intronic & 0.14 & 0.59 & $0.37(0.21-0.64)$ & $3.72 \times 10^{-04}$ & $4.5 \times 10^{-4}$ \\
\hline rs7025331 & 9 p24 & 9 & $\mathrm{C}$ & intergenic & 0.40 & 0.57 & $0.46(0.30-0.71)$ & $3.72 \times 10^{-04}$ & $4.5 \times 10^{-4}$ \\
\hline rs7917910 & $10 q 26$ & 10 & A & intronic & 0.24 & 0.33 & $0.43(0.27-0.69)$ & $3.74 \times 10^{-04}$ & $6.0 \times 10^{-4}$ \\
\hline rs349707 & $3 \mathrm{p} 25$ & 3 & G & intergenic & 0.43 & 0.57 & $0.51(0.35-0.74)$ & $3.76 \times 10^{-04}$ & $2.0 \times 10^{-4}$ \\
\hline rs61748749 & N4BP2 & 4 & G & coding & 0.16 & 0.10 & $0.10(0.03-0.36)$ & $3.93 \times 10^{-04}$ & $1.5 \times 10^{-4}$ \\
\hline rs9873177 & PDIA5 & 3 & $\mathrm{C}$ & intronic & 0.06 & 0.18 & $0.30(0.16-0.59)$ & $4.03 \times 10^{-04}$ & $2.0 \times 10^{-4}$ \\
\hline rs739924 & SPATA20 & 17 & $\mathrm{C}$ & intronic & 0.25 & 0.43 & $0.45(0.29-0.70)$ & $4.04 \times 10^{-04}$ & $4.0 \times 10^{-4}$ \\
\hline rs 12505859 & $4 q 35$ & 4 & G & intergenic & 0.39 & 0.30 & $2.07(1.38-3.11)$ & $4.10 \times 10^{-04}$ & $4.0 \times 10^{-4}$ \\
\hline rs7322477 & LINC00284 & 13 & $\mathrm{C}$ & intronic & 0.14 & 0.27 & $0.39(0.23-0.66)$ & $4.15 \times 10^{-04}$ & $6.0 \times 10^{-4}$ \\
\hline rs 1978759 & MGMT & 10 & A & intronic & 0.36 & 0.36 & $0.44(0.27-0.69)$ & $4.17 \times 10^{-04}$ & $6.0 \times 10^{-4}$ \\
\hline rs 725038 & ACACA & 17 & $\mathrm{C}$ & intronic & 0.32 & 0.55 & $0.51(0.35-0.74)$ & $4.18 \times 10^{-04}$ & $5.0 \times 10^{-4}$ \\
\hline
\end{tabular}




\begin{tabular}{|c|c|c|c|c|c|c|c|c|c|}
\hline \multirow[b]{2}{*}{ SNP } & \multirow[b]{2}{*}{ Gene/region } & \multirow[b]{2}{*}{$\mathrm{Chr}^{\dagger}$} & \multirow[b]{2}{*}{$\begin{array}{l}\text { Minor } \\
\text { allele }\end{array}$} & \multirow[b]{2}{*}{ Feature } & \multicolumn{2}{|c|}{$\begin{array}{c}\text { Minor allele } \\
\text { frequency }\end{array}$} & \multirow[b]{2}{*}{$\mathrm{OR}_{\text {allele }}(95 \% \mathrm{CI}) *$} & \multirow[b]{2}{*}{ P value** } & \multirow[b]{2}{*}{$\begin{array}{c}\text { P value } \\
\text { (permutation) }^{\dagger \dagger}\end{array}$} \\
\hline & & & & & Males & Females & & & \\
\hline rs 729867 & CSRNP3 & 2 & $\mathrm{~T}$ & intronic & 0.18 & 0.09 & $2.92(1.61-5.31)$ & $4.33 \times 10^{-04}$ & $3.0 \times 10^{-4}$ \\
\hline rs 4875742 & CSMD1 & 8 & $\mathrm{C}$ & intronic & 0.30 & 0.23 & $0.36(0.20-0.64)$ & $4.63 \times 10^{-04}$ & $2.0 \times 10^{-4}$ \\
\hline rs1720326 & $11 \mathrm{q} 24$ & 11 & $\mathrm{G}$ & intergenic & 0.49 & 0.25 & $2.18(1.41-3.39)$ & $5.06 \times 10^{-04}$ & $2.0 \times 10^{-4}$ \\
\hline rs 1713676 & $11 q 23$ & 11 & $\mathrm{~T}$ & intergenic & 0.43 & 0.39 & $2.21(1.41-3.45)$ & $5.10 \times 10^{-04}$ & $6.0 \times 10^{-4}$ \\
\hline rs 3857193 & SORCS2 & 4 & $\mathrm{C}$ & intronic & 0.25 & 0.36 & $0.46(0.29-0.71)$ & $5.18 \times 10^{-04}$ & $8.0 \times 10^{-4}$ \\
\hline rs6987111 & CCDC25 & 8 & $\mathrm{~A}$ & intronic & 0.15 & 0.05 & $3.91(1.81-8.46)$ & $5.21 \times 10^{-04}$ & $4.0 \times 10^{-4}$ \\
\hline rs4801931 & ZNF611 & 19 & $\mathrm{C}$ & intronic & 0.28 & 0.54 & $0.49(0.32-0.73)$ & $5.24 \times 10^{-04}$ & $6.0 \times 10^{-4}$ \\
\hline rs 12940887 & ZNF652 & 17 & $\mathrm{~T}$ & intronic & 0.30 & 0.19 & $2.27(1.43-3.61)$ & $5.28 \times 10^{-04}$ & $2.0 \times 10^{-4}$ \\
\hline rs7225787 & ZNF652 & 17 & $\mathrm{C}$ & intronic & 0.34 & 0.27 & $2.09(1.38-3.16)$ & $5.28 \times 10^{-04}$ & $3.0 \times 10^{-4}$ \\
\hline rs11079867 & $17 q 21$ & 17 & $\mathrm{C}$ & intergenic & 0.44 & 0.28 & $2.07(1.37-3.12)$ & $5.32 \times 10^{-04}$ & $7.0 \times 10^{-4}$ \\
\hline rs4680582 & SMC4 & 3 & A & intronic & 0.16 & 0.06 & $3.33(1.69-6.58)$ & $5.33 \times 10^{-04}$ & $5.0 \times 10^{-4}$ \\
\hline rs1429143 & $6 \mathrm{p} 12$ & 6 & $\mathrm{G}$ & intergenic & 0.37 & 0.34 & $0.43(0.27-0.69)$ & $5.39 \times 10^{-04}$ & $3.0 \times 10^{-4}$ \\
\hline rs10506041 & $12 \mathrm{p} 11.2$ & 12 & A & intergenic & 0.46 & 0.38 & $2.16(1.40-3.34)$ & $5.47 \times 10^{-04}$ & $4.0 \times 10^{-4}$ \\
\hline rs1915337 & $3 q 13.1$ & 3 & A & intergenic & 0.10 & 0.24 & $0.39(0.22-0.66)$ & $5.50 \times 10^{-04}$ & $7.0 \times 10^{-4}$ \\
\hline rs6962352 & HIP1 & 7 & $\mathrm{G}$ & intronic & 0.21 & 0.45 & $0.49(0.33-0.74)$ & $5.52 \times 10^{-04}$ & $5.0 \times 10^{-4}$ \\
\hline rs634413 & LINC00544 & 13 & A & intronic & 0.30 & 0.59 & $0.50(0.34-0.74)$ & $5.56 \times 10^{-04}$ & $4.0 \times 10^{-4}$ \\
\hline rs 4074605 & SYNRG & 17 & $\mathrm{C}$ & intronic & 0.21 & 0.1 & $2.84(1.57-5.14)$ & $5.66 \times 10^{-04}$ & $5.0 \times 10^{-4}$ \\
\hline rs7407281 & $18 \mathrm{p} 21$ & 18 & A & intergenic & 0.23 & 0.1 & $2.69(1.53-4.74)$ & $6.04 \times 10^{-04}$ & $7.0 \times 10^{-4}$ \\
\hline rs10778311 & TXNRD1 & 12 & G & intronic & 0.26 & 0.24 & $2.09(1.37-3.20)$ & $6.06 \times 10^{-04}$ & $4.0 \times 10^{-4}$ \\
\hline rs7492685 & $14 q 21$ & 14 & $\mathrm{C}$ & intergenic & 0.16 & 0.11 & $0.10(0.03-0.39)$ & $6.08 \times 10^{-04}$ & $3.0 \times 10^{-4}$ \\
\hline rs4246284 & $15 q 26$ & 15 & $\mathrm{C}$ & intergenic & 0.16 & 0.26 & $0.40(0.24-0.67)$ & $6.12 \times 10^{-04}$ & $2.0 \times 10^{-4}$ \\
\hline
\end{tabular}


Appendix Table 2 continued....

\begin{tabular}{|c|c|c|c|c|c|c|c|c|c|}
\hline \multicolumn{9}{|c|}{} & \multicolumn{2}{c|}{$\begin{array}{c}\text { Minor allele } \\
\text { frequency }\end{array}$} & \multicolumn{2}{l|}{} \\
\hline SNP & Gene/region & Chr $^{\dagger}$ & $\begin{array}{c}\text { Minor } \\
\text { allele }\end{array}$ & Feature & Males & Females & OR $_{\text {allele }}$ (95\% CI)* & P value** $^{* *}$ & $\begin{array}{c}\text { P value } \\
\text { (permutation) }\end{array}$ \\
\hline rs10128580 & ZBTB16 & 11 & $\mathrm{G}$ & intronic & 0.21 & 0.09 & $3.04(1.61-5.74)$ & $6.13 \times 10^{-04}$ & $7.0 \times 10^{-4}$ \\
\hline rs7243843 & DLGAP1 & 18 & $\mathrm{~T}$ & intronic & 0.28 & 0.55 & $0.51(0.34-0.75)$ & $6.20 \times 10^{-04}$ & $8.0 \times 10^{-4}$ \\
\hline rs1602491 & $4 \mathrm{q} 34$ & 4 & $\mathrm{G}$ & intergenic & 0.36 & 0.21 & $2.24(1.41-3.55)$ & $6.23 \times 10^{-04}$ & $7.0 \times 10^{-4}$ \\
\hline rs17439813 & $12 \mathrm{p} 11.2$ & 12 & $\mathrm{C}$ & intergenic & 0.44 & 0.27 & $2.17(1.39-3.37)$ & $6.23 \times 10^{-04}$ & $7.0 \times 10^{-4}$ \\
\hline rs9373051 & EYA4 & 6 & $\mathrm{~T}$ & intronic & 0.25 & 0.07 & $3.18(1.64-6.16)$ & $6.26 \times 10^{-04}$ & $6.0 \times 10^{-4}$ \\
\hline rs309026 & $12 \mathrm{p} 11.2$ & 12 & $\mathrm{~T}$ & intergenic & 0.46 & 0.29 & $2.07(1.36-3.15)$ & $6.30 \times 10^{-4}$ & $1.0 \times 10^{-4}$ \\
\hline rs11011225 & $10 \mathrm{p} 11.2$ & 10 & $\mathrm{~T}$ & intergenic & 0.07 & 0.18 & $0.32(0.17-0.62)$ & $6.62 \times 10^{-4}$ & $2.0 \times 10^{-4}$ \\
\hline rs12904345 & $15 \mathrm{q} 26.2$ & 15 & $\mathrm{C}$ & intergenic & 0.02 & 0.24 & $0.39(0.23-0.67)$ & $6.64 \times 10^{-4}$ & $3.0 \times 10^{-4}$ \\
\hline rs7700370 & ADAMTS12 & 5 & $\mathrm{C}$ & intronic & 0.37 & 0.21 & $2.18(1.39-3.40)$ & $6.69 \times 10^{-4}$ & $4.0 \times 10^{-4}$ \\
\hline rs910850 & DOK5 & 20 & $\mathrm{G}$ & intronic & 0.42 & 0.26 & $2.08(1.36-3.16)$ & $6.73 \times 10^{-4}$ & $4.0 \times 10^{-4}$ \\
\hline rs12822012 & $12 \mathrm{q} 13.1$ & 12 & $\mathrm{C}$ & intergenic & 0.09 & 0.20 & $0.34(0.18-0.63)$ & $6.81 \times 10^{-4}$ & $9.0 \times 10^{-4}$ \\
\hline rs7237664 & $18 \mathrm{q} 11.2$ & 18 & $\mathrm{~T}$ & intergenic & 0.25 & 0.54 & $0.51(0.34-0.75)$ & $6.86 \times 10^{-4}$ & $4.0 \times 10^{-4}$ \\
\hline rs1821567 & C8orf34 & 8 & $\mathrm{C}$ & intronic & 0.31 & 0.29 & $2.13(1.38-3.31)$ & $7.07 \times 10^{-4}$ & $5.0 \times 10^{-4}$ \\
\hline rs1477656 & $5 \mathrm{q} 21$ & 5 & $\mathrm{~T}$ & intergenic & 0.23 & 0.10 & $2.75(1.53-4.95)$ & $7.09 \times 10^{-4}$ & $3.0 \times 10^{-4}$ \\
\hline rs7163190 & $15 \mathrm{q} 14$ & 15 & $\mathrm{G}$ & intergenic & 0.20 & 0.36 & $0.47(0.30-0.72)$ & $7.12 \times 10^{-4}$ & $7.0 \times 10^{-4}$ \\
\hline rs11648233 & HSD17B2 & 16 & $\mathrm{C}$ & intronic & 0.26 & 0.44 & $0.49(0.32-0.74)$ & $7.15 \times 10^{-4}$ & $5.0 \times 10^{-4}$ \\
\hline
\end{tabular}

${ }^{*} \mathrm{OR}_{\text {interaction }}$ per allele $\left(\mathrm{OR}_{\text {allele }}\right)$ for the additive model; $* * P$ value adjusted for genomic control; ${ }^{\dagger \dagger} \mathrm{P}$ value permutation $=$ point wise $\mathrm{P}$ value from maxT permutation analysis after 10,000 permutation

${ }^{\dagger} \mathrm{Chr}=$ Chromosome, $\mathrm{SNP}=$ single nucleotide polymorphism 
Appendix Table 3: List of single nucleotide polymorphisms that showed genderspecific associations based on the $P$ value and were statistically correlated

\begin{tabular}{|c|c|c|c|}
\hline Chr A & SNP 1 & Chr B & SNP 2 \\
\hline 2 & rs7583193 & 2 & rs 17027254 \\
\hline 2 & rs6732320 & 2 & rs1899286 \\
\hline 2 & rs6732320 & 2 & rs11687208 \\
\hline 2 & rs1899286 & 2 & rs11687208 \\
\hline 2 & rs563694 & 2 & rs552976 \\
\hline 2 & rs17027254 & 2 & rs6732320 \\
\hline 2 & rs7583193 & 2 & rs6732320 \\
\hline 2 & rs7583193 & 2 & rs1899286 \\
\hline 2 & rs17027254 & 2 & rs11687208 \\
\hline 2 & rs17027254 & 2 & rs1899286 \\
\hline 2 & rs560887 & 2 & rs563694 \\
\hline 2 & rs7583193 & 2 & rs11687208 \\
\hline 4 & rs13107783 & 4 & rs4470624 \\
\hline 4 & rs61748746 & 4 & rs61748749 \\
\hline 4 & rs1550017 & 4 & rs 12054636 \\
\hline 12 & rs17439813 & 12 & rs11049786 \\
\hline 12 & rs17439813 & 12 & rs1849374 \\
\hline 12 & rs11049786 & 12 & rs1849374 \\
\hline 13 & rs4942264 & 13 & rs7322477 \\
\hline 13 & rs634413 & 13 & rs6490437 \\
\hline 15 & rs4246284 & 15 & rs 12904345 \\
\hline 17 & rs8081866 & 17 & rs2542653 \\
\hline 17 & rs8081866 & 17 & rs 2542660 \\
\hline 17 & rs8081866 & 17 & rs 12948120 \\
\hline 17 & rs2542653 & 17 & rs2542660 \\
\hline 17 & rs2542653 & 17 & rs 12948120 \\
\hline 17 & rs2542660 & 17 & rs 12948120 \\
\hline 17 & rs4794031 & 17 & rs7225787 \\
\hline 17 & rs11079867 & 17 & rs7225787 \\
\hline 17 & rs4794031 & 17 & rs11079867 \\
\hline 17 & rs12940887 & 17 & rs7225787 \\
\hline 17 & rs725038 & 17 & rs 2542653 \\
\hline
\end{tabular}


Appendix Table 3 continued....

\begin{tabular}{|c|c|c|c|}
\hline Chr A & SNP 1 & Chr B & SNP 2 \\
\hline 17 & rs725038 & 17 & rs2542660 \\
\hline 17 & rs4794031 & 17 & rs12940887 \\
\hline
\end{tabular}

$\mathrm{Chr}=$ chromosome; $\mathrm{SNP}=$ single nucleotide polymorphism

Note: Chromosome A and Chromosome B are same chromosome. SNP1 and SNP2 and statistically correlated SNPS 
Appendix Table 4: List of single nucleotide polymorphisms that showed genderspecific associations based on effect size and were statistically correlated

\begin{tabular}{|c|c|c|c|}
\hline Chr A & SNP 1 & Chr B & SNP 2 \\
\hline 2 & rs1049894 & 2 & rs11904365 \\
\hline 4 & rs10003958 & 4 & rs9993832 \\
\hline 6 & rs130069 & 6 & rs6929434 \\
\hline 6 & rs12722039 & 6 & rs12722042 \\
\hline 6 & rs17080410 & 6 & rs61742396 \\
\hline 7 & rs4496864 & 7 & rs35912436 \\
\hline 7 & rs4496864 & 7 & rs2160271 \\
\hline 7 & rs35912436 & 7 & rs2160271 \\
\hline 8 & rs34265667 & 8 & rs34664882 \\
\hline 8 & rs11992342 & 8 & rs36041430 \\
\hline 8 & rs36041430 & 8 & rs7829965 \\
\hline 8 & rs11992342 & 8 & rs7829965 \\
\hline 9 & rs3847262 & 9 & rs2890707 \\
\hline 9 & rs4645656 & 9 & rs7848710 \\
\hline 9 & rs4645656 & 9 & rs7037100 \\
\hline 9 & rs4645656 & 9 & rs6477733 \\
\hline 9 & rs 7848710 & 9 & rs7037100 \\
\hline 9 & rs 7848710 & 9 & rs6477733 \\
\hline 9 & rs 7037100 & 9 & rs6477733 \\
\hline 19 & rs16973890 & 19 & rs117923468 \\
\hline 19 & rs16973890 & 19 & rs182319278 \\
\hline 19 & rs117923468 & 19 & rs182319278 \\
\hline
\end{tabular}

$\mathrm{Chr}=$ chromosome; $\mathrm{SNP}=$ single nucleotide polymorphism

Note: Chromosome A and Chromosome B are same chromosome. SNP1 and SNP2 and statistically correlated SNPs 
Appendix Table 5: Top one hundred SNPs associated with childhood ALL risk with gender-specificity, results based on the highest effect sizes $(P<\mathbf{0 . 0 5})$

\begin{tabular}{|c|c|c|c|c|c|c|c|c|c|}
\hline \multirow[b]{2}{*}{ SNP } & \multirow[b]{2}{*}{ Gene/region } & \multirow[b]{2}{*}{ Chr } & \multirow[b]{2}{*}{$\begin{array}{l}\text { Minor } \\
\text { allele }\end{array}$} & \multirow[b]{2}{*}{ Feature } & \multicolumn{2}{|c|}{$\begin{array}{l}\text { Minor allele } \\
\text { frequency }\end{array}$} & \multirow[b]{2}{*}{$O R_{\text {allele }}(95 \% \mathrm{CI}) *$} & \multirow[b]{2}{*}{$P$ value $* *$} & \multirow[b]{2}{*}{$\begin{array}{c}\text { P value } \\
\text { (permutation) }^{\dagger \dagger}\end{array}$} \\
\hline & & & & & Males & Females & & & \\
\hline rs 12722042 & HLA-DQA1 & 6 & $\mathrm{G}$ & coding & 0.07 & 0.005 & $15.68(2.03-121.2)$ & 0.008 & 0.001 \\
\hline rs12722039 & HLA-DQA1 & 6 & $\mathrm{~A}$ & coding & 0.07 & 0.005 & $14.87(1.94-114.0)$ & 0.009 & 0.002 \\
\hline rs11992342 & ADAM28 & 8 & $\mathrm{~T}$ & intron & 0.07 & 0.005 & $14.73(1.92-113.9)$ & 0.01 & 0.001 \\
\hline rs80040922 & UMODL1 & 21 & $\mathrm{~A}$ & coding & 0.06 & 0.005 & $13.68(1.77-105.6)$ & 0.01 & 0.004 \\
\hline rs61753605 & LOC100996481 & 6 & $\mathrm{C}$ & coding & 0.06 & 0.005 & $13.38(1.73-103.3)$ & 0.01 & 0.004 \\
\hline rs35665085 & CECR5 & 22 & $\mathrm{~A}$ & coding & 0.06 & 0.005 & $12.64(1.63-98.00)$ & 0.02 & 0.006 \\
\hline rs143021649 & CNTN3 & 3 & $\mathrm{~T}$ & coding & 0.06 & 0.005 & $11.62(1.49-90.58)$ & 0.02 & 0.009 \\
\hline rs6795524 & PROS1 & 3 & $\mathrm{G}$ & intron & 0.05 & 0.005 & $10.63(1.36-83.31)$ & 0.02 & 0.006 \\
\hline rs10003468 & $4 q 28.1$ & 4 & $\mathrm{C}$ & intergenic & 0.05 & 0.005 & $10.63(1.36-83.31)$ & 0.02 & 0.005 \\
\hline rs10131142 & $14 q 11.2$ & 14 & $\mathrm{~A}$ & intergenic & 0.07 & 0.01 & $7.82(1.76-34.78)$ & 0.007 & 0.002 \\
\hline rs3732042 & CNRIP1 & 2 & $\mathrm{~A}$ & 3utr & 0.08 & 0.01 & $7.50(1.71-32.96)$ & 0.008 & 0.002 \\
\hline rs34022679 & GRK4 & 4 & $\mathrm{C}$ & coding & 0.07 & 0.01 & $6.98(1.58-30.76)$ & 0.01 & 0.003 \\
\hline rs 7829965 & ADAM28 & 8 & $\mathrm{~A}$ & coding & 0.06 & 0.01 & $6.77(1.51-30.38)$ & 0.01 & 0.007 \\
\hline rs7097067 & $10 \mathrm{q} 22.1$ & 10 & $\mathrm{~T}$ & intergenic & 0.06 & 0.01 & $6.77(1.51-30.38)$ & 0.01 & 0.01 \\
\hline rs35912436 & $7 \mathrm{p} 21.3$ & 7 & $\mathrm{C}$ & intergenic & 0.08 & 0.01 & $6.69(1.54-29.00)$ & 0.01 & 0.003 \\
\hline rs2160271 & $7 \mathrm{p} 21.3$ & 7 & $\mathrm{C}$ & intergenic & 0.08 & 0.01 & $6.69(1.54-29.00)$ & 0.01 & 0.003 \\
\hline rs17588454 & $13 q 12.3$ & 13 & $\mathrm{G}$ & intergenic & 0.07 & 0.01 & $6.47(1.46-28.61)$ & 0.01 & 0.006 \\
\hline rs368390 & C18orf42 & 18 & $\mathrm{~T}$ & intron & 0.09 & 0.02 & $6.25(1.80-21.76)$ & 0.004 & 0.001 \\
\hline rs17080410 & PLEKHG1 & 6 & $\mathrm{C}$ & coding & 0.06 & 0.01 & $6.25(1.38-28.25)$ & 0.02 & 0.01 \\
\hline
\end{tabular}


Appendix Table 5 continued....

\begin{tabular}{|c|c|c|c|c|c|c|c|c|c|}
\hline \multirow[b]{2}{*}{ SNP } & \multirow[b]{2}{*}{ Gene/region } & \multirow[b]{2}{*}{ Chr } & \multirow[b]{2}{*}{$\begin{array}{l}\text { Minor } \\
\text { allele }\end{array}$} & \multirow[b]{2}{*}{ Feature } & \multicolumn{2}{|c|}{$\begin{array}{l}\text { Minor allele } \\
\text { frequency }\end{array}$} & \multirow[b]{2}{*}{$\mathrm{OR}_{\text {allele }}(95 \% \mathrm{CI})^{*}$} & \multirow[b]{2}{*}{$P$ value** } & \multirow[b]{2}{*}{$\begin{array}{c}\text { P value } \\
\text { (permutation) }\end{array}$} \\
\hline & & & & & Males & Females & & & \\
\hline rs61742396 & PLEKHG1 & 6 & $\mathrm{~A}$ & coding & 0.06 & 0.01 & $6.25(1.38-28.25)$ & 0.02 & 0.01 \\
\hline rs35672330 & EXO5 & 1 & $\mathrm{C}$ & coding & 0.06 & 0.01 & $5.98(1.35-26.50)$ & 0.02 & 0.01 \\
\hline rs1503292 & TLL1 & 4 & $\mathrm{C}$ & intron & 0.06 & 0.01 & $5.97(1.35-26.47)$ & 0.02 & 0.01 \\
\hline rs1978526 & $2 \mathrm{q} 24.3$ & 2 & $\mathrm{G}$ & intergenic & 0.06 & 0.01 & $5.91(1.33-26.19)$ & 0.02 & 0.01 \\
\hline rs6887604 & $5 \mathrm{q} 12.3$ & 5 & $\mathrm{~T}$ & intergenic & 0.06 & 0.01 & $5.75(1.26-26.16)$ & 0.02 & 0.01 \\
\hline rs 10063286 & $5 \mathrm{q} 35.2$ & 5 & $\mathrm{G}$ & intergenic & 0.06 & 0.01 & $5.75(1.26-26.16)$ & 0.02 & 0.01 \\
\hline rs16879126 & NT5C3A & 7 & $\mathrm{C}$ & intron & 0.06 & 0.01 & $5.75(1.26-26.16)$ & 0.02 & 0.009 \\
\hline rs34265667 & ANK1 & 8 & A & coding & 0.06 & 0.01 & $5.75(1.26-26.16)$ & 0.02 & 0.009 \\
\hline rs34664882 & $A N K 1$ & 8 & $\mathrm{~A}$ & coding & 0.06 & 0.01 & $5.75(1.26-26.16)$ & 0.02 & 0.009 \\
\hline rs61731389 & PCDH15 & 10 & $\mathrm{C}$ & coding & 0.06 & 0.01 & $5.75(1.26-26.16)$ & 0.02 & 0.01 \\
\hline rs 4764332 & $12 \mathrm{p} 13.2$ & 12 & $\mathrm{~T}$ & intergenic & 0.06 & 0.01 & $5.75(1.26-26.16)$ & 0.02 & 0.01 \\
\hline rs4496864 & $7 \mathrm{p} 21.3$ & 7 & $\mathrm{~T}$ & intergenic & 0.07 & 0.01 & $5.72(1.32-24.80)$ & 0.02 & 0.01 \\
\hline rs6936494 & $6 q 15$ & 6 & $\mathrm{~T}$ & intergenic & 0.11 & 0.02 & $5.67(1.91-16.83)$ & 0.002 & $7.5 \times 10^{-4}$ \\
\hline rs3847262 & TPD52L3 & 9 & $\mathrm{~T}$ & coding & 0.06 & 0.01 & $5.49(1.24-24.43)$ & 0.03 & 0.009 \\
\hline rs2890707 & TPD52L3 & 9 & A & 3utr & 0.06 & 0.01 & $5.49(1.24-24.43)$ & 0.03 & 0.009 \\
\hline rs7626907 & $3 \mathrm{p} 24.3$ & 3 & A & intergenic & 0.10 & 0.02 & $5.37(1.81-15.99)$ & 0.003 & 0.001 \\
\hline rs2229528 & ACAA1 & 3 & G & coding & 0.05 & 0.01 & $5.26(1.15-24.11)$ & 0.03 & 0.02 \\
\hline rs9842465 & SCHIP1 & 3 & $\mathrm{~T}$ & intron & 0.05 & 0.01 & $5.26(1.15-24.11)$ & 0.03 & 0.01 \\
\hline rs6796558 & $3 q 28$ & 3 & $\mathrm{~A}$ & intergenic & 0.05 & 0.01 & $5.26(1.15-24.11)$ & 0.03 & 0.02 \\
\hline rs55882716 & MAST4 & 5 & $\mathrm{~T}$ & coding & 0.05 & 0.01 & $5.26(1.15-24.11)$ & 0.03 & 0.01 \\
\hline rs 1030270 & CYFIP2 & 5 & A & intron & 0.05 & 0.01 & $5.26(1.15-24.11)$ & 0.03 & 0.01 \\
\hline
\end{tabular}


Appendix Table 5 continued....

\begin{tabular}{|c|c|c|c|c|c|c|c|c|c|}
\hline \multirow[b]{2}{*}{ SNP } & \multirow[b]{2}{*}{ Gene/region } & \multirow[b]{2}{*}{ Chr } & \multirow[b]{2}{*}{$\begin{array}{l}\text { Minor } \\
\text { allele }\end{array}$} & \multirow[b]{2}{*}{ Feature } & \multicolumn{2}{|c|}{$\begin{array}{l}\text { Minor allele } \\
\text { frequency }\end{array}$} & \multirow[b]{2}{*}{$\mathrm{OR}_{\text {allele }}(95 \% \mathrm{CI})^{*}$} & \multirow[b]{2}{*}{$P$ value** } & \multirow[b]{2}{*}{$\begin{array}{c}\text { P value } \\
\text { (permutation) }^{\dagger}\end{array}$} \\
\hline & & & & & Males & Females & & & \\
\hline rs205944 & SLC35F1 & 6 & $\mathrm{~A}$ & intron & 0.05 & 0.01 & $5.26(1.15-24.11)$ & 0.03 & 0.02 \\
\hline rs 381783 & IL20RA & 6 & $\mathrm{~T}$ & intron & 0.05 & 0.01 & $5.26(1.15-24.11)$ & 0.03 & 0.01 \\
\hline rs11980237 & KDELR2 & 7 & $\mathrm{~A}$ & intron & 0.05 & 0.01 & $5.26(1.15-24.11)$ & 0.03 & 0.01 \\
\hline rs720872 & $7 \mathrm{p} 21.2$ & 7 & $\mathrm{C}$ & intergenic & 0.05 & 0.01 & $5.26(1.15-24.11)$ & 0.03 & 0.01 \\
\hline rs147596194 & RGS3 & 9 & $\mathrm{~T}$ & intron & 0.05 & 0.01 & $5.26(1.15-24.11)$ & 0.03 & 0.01 \\
\hline rs61745456 & MAMDC4 & 9 & $\mathrm{~T}$ & coding & 0.05 & 0.01 & $5.26(1.15-24.11)$ & 0.03 & 0.01 \\
\hline rs117923468 & WDR87 & 19 & A & coding & 0.05 & 0.01 & $5.26(1.15-24.11)$ & 0.03 & 0.01 \\
\hline rs182319278 & WDR87 & 19 & $\mathrm{C}$ & coding & 0.05 & 0.01 & $5.26(1.15-24.11)$ & 0.03 & 0.01 \\
\hline rs9305777 & $21 \mathrm{q} 21.1$ & 21 & $\mathrm{~T}$ & intergenic & 0.05 & 0.01 & $5.26(1.15-24.11)$ & 0.03 & 0.02 \\
\hline rs8129749 & $21 \mathrm{q} 21.2$ & 21 & $\mathrm{~T}$ & intergenic & 0.05 & 0.01 & $5.26(1.15-24.11)$ & 0.03 & 0.01 \\
\hline rs985456 & $21 \mathrm{q} 21.3$ & 21 & $\mathrm{C}$ & intergenic & 0.05 & 0.01 & $5.26(1.15-24.11)$ & 0.03 & 0.01 \\
\hline rs16863233 & $L P P$ & 3 & $\mathrm{C}$ & intron & 0.07 & 0.02 & $5.16(1.46-18.18)$ & 0.01 & 0.008 \\
\hline rs7037100 & ZNF782 & 9 & $\mathrm{C}$ & intron & 0.07 & 0.02 & $5.16(1.46-18.18)$ & 0.01 & 0.008 \\
\hline rs7630157 & EGFEM1P & 3 & $\mathrm{C}$ & intron & 0.06 & 0.01 & $5.07(1.14-22.62)$ & 0.03 & 0.01 \\
\hline rs62640905 & OSGIN1 & 16 & $\mathrm{C}$ & coding & 0.06 & 0.01 & $5.02(1.13-22.40)$ & 0.03 & 0.01 \\
\hline rs16973890 & ZNF571/ZNF540 & 19 & $\mathrm{C}$ & intron & 0.06 & 0.01 & $5.02(1.13-22.40)$ & 0.03 & 0.01 \\
\hline rs35693261 & MTG2 & 20 & $\mathrm{~T}$ & coding & 0.06 & 0.01 & $5.02(1.13-22.40)$ & 0.03 & 0.01 \\
\hline rs7539097 & $T N R$ & 1 & G & intron & 0.08 & 0.02 & $5.00(1.44-17.32)$ & 0.01 & 0.005 \\
\hline rs9493135 & $6 \mathrm{q} 23.2$ & 6 & A & intergenic & 0.08 & 0.01 & $4.94(1.43-17.14)$ & 0.01 & 0.005 \\
\hline rs6722598 & MFF & 2 & $\mathrm{~A}$ & 5upstream & 0.09 & 0.02 & $4.92(1.63-14.89)$ & 0.005 & 0.002 \\
\hline rs1049894 & PSME4 & 2 & $\mathrm{~T}$ & coding & 0.07 & 0.02 & $4.81(1.36-17.03)$ & 0.02 & 0.01 \\
\hline
\end{tabular}


Appendix Table 5 continued....

\begin{tabular}{|c|c|c|c|c|c|c|c|c|c|}
\hline & & & & & $\begin{array}{c}\text { Min } \\
\text { frec }\end{array}$ & $\begin{array}{l}\text { allele } \\
\text { ency }\end{array}$ & & & \\
\hline SNP & Gene/region & Chr & $\begin{array}{c}\text { Minor } \\
\text { allele }\end{array}$ & Feature & Males & Females & $\mathrm{OR}_{\text {allele }}(95 \% \mathrm{CI})^{*}$ & $P$ value** & $\begin{array}{c}\text { P value } \\
\text { (permutation) }^{\dagger \dagger}\end{array}$ \\
\hline rs11904365 & $2 \mathrm{p} 16.2$ & 2 & $\mathrm{C}$ & intergenic & 0.07 & 0.02 & $4.81(1.36-17.03)$ & 0.02 & 0.009 \\
\hline rs10003958 & $R A B 28$ & 4 & $\mathrm{C}$ & intron & 0.07 & 0.02 & $4.81(1.36-17.03)$ & 0.02 & 0.01 \\
\hline rs9993832 & $R A B 28$ & 4 & $\mathrm{~T}$ & intron & 0.07 & 0.02 & $4.81(1.36-17.03)$ & 0.02 & 0.01 \\
\hline rs36041430 & ADAM28 & 8 & $\mathrm{~A}$ & coding & 0.07 & 0.02 & $4.81(1.36-17.03)$ & 0.02 & 0.01 \\
\hline rs78888579 & CROCC & 1 & A & coding & 0.05 & 0.01 & $4.77(1.03-22.10)$ & 0.05 & 0.01 \\
\hline rs 10218650 & $1 \mathrm{q} 31.3$ & 1 & $\mathrm{~T}$ & intergenic & 0.05 & 0.01 & $4.77(1.03-22.10)$ & 0.05 & 0.04 \\
\hline rs13405142 & $2 \mathrm{p} 22.1$ & 2 & $\mathrm{~T}$ & intergenic & 0.05 & 0.01 & $4.77(1.03-22.10)$ & 0.05 & 0.03 \\
\hline rs10194197 & $2 \mathrm{p} 12$ & 2 & $\mathrm{~T}$ & intergenic & 0.05 & 0.01 & $4.77(1.03-22.10)$ & 0.05 & 0.04 \\
\hline rs61741435 & CCDC173 & 2 & $\mathrm{~T}$ & coding & 0.05 & 0.01 & $4.77(1.03-22.10)$ & 0.05 & 0.02 \\
\hline rs4856270 & CADM2 & 3 & $\mathrm{~T}$ & intron & 0.05 & 0.01 & $4.77(1.03-22.10)$ & 0.05 & 0.02 \\
\hline rs2165084 & $4 \mathrm{q} 31.3$ & 4 & $\mathrm{G}$ & intergenic & 0.05 & 0.01 & $4.77(1.03-22.10)$ & 0.05 & 0.02 \\
\hline rs 7703414 & $5 \mathrm{p} 13.1$ & 5 & $\mathrm{~A}$ & intergenic & 0.05 & 0.01 & $4.77(1.03-22.10)$ & 0.05 & 0.03 \\
\hline rs11751765 & TRERF1 & 6 & $\mathrm{~T}$ & coding & 0.05 & 0.01 & $4.77(1.03-22.10)$ & 0.05 & 0.04 \\
\hline rs7797334 & $7 \mathrm{p} 21.2$ & 7 & $\mathrm{C}$ & intergenic & 0.05 & 0.01 & $4.77(1.03-22.10)$ & 0.05 & 0.02 \\
\hline rs4278110 & $7 \mathrm{p} 12.2$ & 7 & A & intergenic & 0.05 & 0.02 & $4.77(1.03-22.10)$ & 0.05 & 0.03 \\
\hline rs11987106 & COLEC10 & 8 & A & intron & 0.05 & 0.01 & $4.77(1.03-22.10)$ & 0.05 & 0.03 \\
\hline rs61735202 & CCIN & 9 & A & coding & 0.05 & 0.01 & $4.77(1.03-22.10)$ & 0.05 & 0.04 \\
\hline rs943811 & $10 \mathrm{q} 26.2$ & 10 & A & intergenic & 0.05 & 0.01 & $4.77(1.03-22.10)$ & 0.05 & 0.03 \\
\hline rs10430621 & $10 \mathrm{q} 26.3$ & 10 & $\mathrm{C}$ & intergenic & 0.05 & 0.01 & $4.77(1.03-22.10)$ & 0.05 & 0.04 \\
\hline rs11182332 & TMEM117 & 12 & $\mathrm{~A}$ & intron & 0.05 & 0.01 & $4.77(1.03-22.10)$ & 0.05 & 0.04 \\
\hline rs2293624 & C12orf73 & 12 & A & coding & 0.05 & 0.01 & $4.77(1.03-22.10)$ & 0.05 & 0.04 \\
\hline
\end{tabular}


Appendix Table 5 continued....

\begin{tabular}{|c|c|c|c|c|c|c|c|c|c|}
\hline & & & & & $\begin{array}{l}\text { Min } \\
\text { fre }\end{array}$ & $\begin{array}{l}\text { allele } \\
\text { iency }\end{array}$ & & & \\
\hline SNP & Gene/region & Chr & $\begin{array}{l}\text { Minor } \\
\text { allele }\end{array}$ & Feature & Males & Females & $\mathrm{OR}_{\text {allele }}(95 \% \mathrm{CI})^{*}$ & $P$ value** & $\begin{array}{c}\text { P value } \\
\text { (permutation) }^{\dagger \dagger}\end{array}$ \\
\hline rs4770684 & PARP4 & 13 & $\mathrm{~A}$ & coding & 0.05 & 0.01 & $4.77(1.03-22.10)$ & 0.05 & 0.03 \\
\hline rs7182827 & UBE3A & 15 & $\mathrm{C}$ & intron & 0.05 & 0.01 & $4.77(1.03-22.10)$ & 0.05 & 0.04 \\
\hline rs12621721 & МАРЗК19 & 2 & A & intron & 0.05 & 0.01 & $4.72(1.02-21.86)$ & 0.05 & 0.03 \\
\hline rs 7275217 & $21 \mathrm{q} 22.1$ & 21 & G & intergenic & 0.05 & 0.01 & $4.72(1.02-21.86)$ & 0.05 & 0.03 \\
\hline rs41299607 & RGSL1 & 1 & A & coding & 0.05 & 0.01 & $4.56(1.02-20.41)$ & 0.05 & 0.03 \\
\hline rs9282777 & LMO2 & 11 & $\mathrm{~A}$ & coding & 0.05 & 0.01 & $4.56(1.02-20.41)$ & 0.05 & 0.02 \\
\hline rs10520291 & $2 \mathrm{p} 12$ & 2 & A & intergenic & 0.08 & 0.02 & $4.52(1.33-15.39)$ & 0.02 & 0.009 \\
\hline rs12037891 & PDE4B & 1 & A & intron & 0.06 & 0.02 & $4.46(1.25-15.91)$ & 0.02 & 0.02 \\
\hline rs73022563 & PLEKHG4B & 5 & $\mathrm{~T}$ & coding & 0.06 & 0.02 & $4.46(1.25-15.91)$ & 0.02 & 0.02 \\
\hline rs130069 & CCHCR1 & 6 & $\mathrm{~T}$ & coding & 0.06 & 0.02 & $4.46(1.25-15.91)$ & 0.02 & 0.02 \\
\hline rs6929434 & TCF19 & 6 & $\bar{A}$ & 5upstream & 0.06 & 0.02 & $4.46(1.25-15.91)$ & 0.02 & 0.02 \\
\hline rs10975823 & KDM4C & 9 & $\mathrm{C}$ & intron & 0.06 & 0.02 & $4.46(1.25-15.91)$ & 0.02 & 0.02 \\
\hline rs4645656 & ZNF782 & 9 & $\mathrm{~T}$ & coding & 0.06 & 0.02 & $4.46(1.25-15.91)$ & 0.02 & 0.02 \\
\hline rs7848710 & ZNF782 & 9 & $\mathrm{~T}$ & intron & 0.06 & 0.02 & $4.46(1.25-15.91)$ & 0.02 & 0.02 \\
\hline rs6477733 & ZNF782 & 9 & G & intron & 0.06 & 0.02 & $4.46(1.25-15.91)$ & 0.02 & 0.02 \\
\hline rs17000817 & $21 \mathrm{q} 21.2$ & 21 & $\mathrm{C}$ & intergenic & 0.08 & 0.02 & $4.45(1.46-13.59)$ & 0.009 & 0.004 \\
\hline rs2633820 & SUMF1 & 3 & A & intron & 0.08 & 0.02 & $4.39(1.32-14.63)$ & 0.02 & 0.007 \\
\hline rs9469223 & $6 \mathrm{p} 24$ & 6 & G & intergenic & 0.08 & 0.02 & $4.39(1.32-14.63)$ & 0.02 & 0.007 \\
\hline
\end{tabular}

${ }^{*} \mathrm{OR}$ interaction per allele $\left(\mathrm{OR}_{\text {allele }}\right)$ for the additive model; ${ }^{* *} P$ value adjusted for genomic control; ${ }^{\dagger \dagger} \mathrm{P}$ value permutation $=$ point wise $\mathrm{P}$ value from maxT permutation analysis after 10,000 permutation

$\mathrm{Chr}=$ Chromosome, $\mathrm{SNP}=$ single nucleotide polymorphism 
Appendix Table 6: Bioinformatic analysis of genetic risk markers for the top one hundred gender-specific associations, results based on the lowest $P$ values

\begin{tabular}{|c|c|c|c|c|c|c|c|}
\hline SNP & Chr & $\underset{\text { score }^{\dagger}}{\text { RegulomeDB }}$ & F-SNP ${ }^{* *}$ & Gene & $\begin{array}{l}\text { Correlated } \\
\text { SNP }\end{array}$ & $\begin{array}{c}\text { RegulomeDB } \\
\text { score }^{\dagger}\end{array}$ & $\begin{array}{c}\text { Gene } \\
\text { (correlated SNPs) }\end{array}$ \\
\hline rs4813720 & 20 & NS & 0.18 & RASSF2 & rs3215695 & $2 \mathrm{~b}$ & RASSF2 \\
\hline rs231237 & 19 & $2 \mathbf{b}$ & 0.12 & HSPB6 & . & . & . \\
\hline rs7323018 & 13 & 5 & NS & LACC1-SMIM2 & . & . & . \\
\hline rs17027254 & 2 & 6 & NS & HNRNPA1P57-LDHAL3 & . & . & . \\
\hline rs7912381 & 2 & NS & 0.1 & TCERG1L-PPP2R2D & . & . & . \\
\hline rs11687208 & 2 & NS & 0.1 & HNRNPA1P57-LDHAL3 & rs1899286 & $3 a$ & HNRNPA1P57-LDHAL3 \\
\hline rs798292 & 7 & 6 & NS & MAGI2 & . & . & . \\
\hline rs13107783 & 4 & 6 & 0.1 & RPL21P46-SLIT2 & rs1905844 & $2 b$ & RPL21P46-SLIT2 \\
\hline rs4470624 & 6 & 6 & 0.1 & RPL21P46-SLIT2 & rs1905844 & $2 \mathrm{~b}$ & RPL21P46-SLIT2 \\
\hline rs 1849374 & 12 & 6 & NS & CCDC91- FAR2 & . & . & . \\
\hline rs12948120 & 17 & 6 & 0.21 & ACACA & rs9330250 & $2 \mathrm{~b}$ & ACACA \\
\hline rs6904762 & 6 & 5 & NS & TMEM244-L3MBTL3 & . & & . \\
\hline rs506389 & 8 & NS & NS & ZNF706-DUXAP2 & . & . & . \\
\hline rs6732320 & 2 & 6 & NS & HNRNPA1P57-LDHAL3 & rs1899286 & $3 a$ & HNRNPA1P57-LDHAL3 \\
\hline rs8081866 & 17 & 4 & 0.21 & ACACA & rs9330250 & $2 \mathrm{~b}$ & ACACA \\
\hline rs349714 & 3 & 5 & 0.1 & SLC6A1-HRH1 & rs13317174 & $2 b$ & SLC6A1-HRH1 \\
\hline rs7583193 & 2 & NS & NS & HNRNPA1P57-LDHAL3 & & & . \\
\hline rs206457 & 18 & 6 & NS & VAPA-APCDD1 & & & . \\
\hline rs7723568 & 5 & 5 & NS & IRX1-LOC101929153 & . & . & . \\
\hline rs2542660 & 17 & 5 & 0.18 & ACACA & rs9330250 & $2 \mathrm{~b}$ & ACACA \\
\hline rs37389 & 5 & NS & 0.27 & $P R L R$ & & & . \\
\hline
\end{tabular}


Appendix Table 6 continued....

\begin{tabular}{|c|c|c|c|c|c|c|c|}
\hline SNP & Chr & $\begin{array}{c}\text { RegulomeDB } \\
\text { score }^{\dagger}\end{array}$ & F-SNP $^{* *}$ & Gene & $\begin{array}{l}\text { Correlated } \\
\text { SNP }\end{array}$ & $\begin{array}{c}\text { RegulomeDB } \\
\text { score }^{\dagger}\end{array}$ & $\begin{array}{c}\text { Gene } \\
\text { (correlated SNPs) }\end{array}$ \\
\hline rs1899286 & 2 & $3 a$ & 0.1 & HNRNPA1P57-LDHAL3 & & & 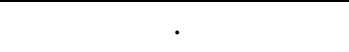 \\
\hline rs9563035 & 13 & NS & NS & LINC00371-FAM124A & . & $\cdot$ & . \\
\hline rs 2542653 & 17 & NS & 0 & ACACA & rs4299194 & $2 \mathrm{~b}$ & ACACA \\
\hline rs 2542653 & 17 & NS & 0 & ACACA & rs9330250 & $2 b$ & ACACA \\
\hline rs6490437 & 13 & 6 & NS & LINC00544-LINC00384 & rs7334559 & $2 \mathrm{~b}$ & LINC00544-LINC00384 \\
\hline rs4794031 & 17 & 5 & 0.5 & PHOSPHO1-ZNF652 & . & . & . \\
\hline rs2544371 & 2 & 6 & NS & LRP2-BBS5 & . & . & . \\
\hline rs 1372166 & 2 & NS & 0.1 & HNRNPA1P57-LDHAL3 & . & . & . \\
\hline rs 7294845 & 12 & 5 & NS & SOX5 & rs11047322 & $3 a$ & SOX5 \\
\hline rs7294845 & 12 & 5 & NS & SOX5 & rs 11047322 & $3 a$ & SOX5 \\
\hline rs12219278 & 10 & 5 & NS & TCERG1L-BNIP3 & . & . & . \\
\hline rs4942264 & 13 & NS & NS & DGKZP1-LINC00284 & rs4418956 & $2 b$ & LINC00284 \\
\hline rs11708505 & 3 & 5 & 0.1 & RYBP-SHQ1 & rs11708482 & $2 \mathrm{~b}$ & RYBP-SHQ1 \\
\hline rs6540610 & 1 & NS & 0.1 & HHAT & . & . & . \\
\hline rs2764928 & 1 & NS & 0.1 & LINC01135-PHBP3 & rs7555848 & $3 a$ & LINC01135-PHBP3 \\
\hline rs1550017 & 4 & 6 & NS & GRIA2-LOC340017 & rs7694905 & $3 a$ & GRIA2-LOC340017 \\
\hline rs12054636 & 4 & NS & NS & GRIA2-LOC340017 & rs7694905 & $3 a$ & GRIA2-LOC340017 \\
\hline rs2764933 & 1 & 5 & NS & LINC01135-PHBP3 & & & . \\
\hline rs468771 & 21 & NS & NS & GRIK1 & . & . & . \\
\hline rs 864674 & 5 & 5 & 0.1 & SUB1-NPR3 & . & . & . \\
\hline rs8106959 & 19 & 1f & NS & KMT2B & rs 10413852 & 1f & UPK1A \\
\hline rs8106959 & 19 & 1f & NS & KMT2B & rs 12461333 & 1f & UPK1A-ZBTB32 \\
\hline
\end{tabular}


Appendix Table 6 continued....

\begin{tabular}{|c|c|c|c|c|c|c|c|}
\hline SNP & Chr & $\begin{array}{c}\text { RegulomeDB } \\
\text { score }^{\dagger}\end{array}$ & F-SNP ${ }^{* *}$ & Gene & $\begin{array}{l}\text { Correlated } \\
\text { SNP }\end{array}$ & $\begin{array}{c}\text { RegulomeDB } \\
\text { score }^{\dagger}\end{array}$ & $\begin{array}{c}\text { Gene } \\
\text { (correlated SNPs) }\end{array}$ \\
\hline rs8106959 & 19 & 1f & NS & КМТ2B & rs12461285 & 1f & UPK1A-ZBТВ32 \\
\hline rs8106959 & 19 & 1f & NS & KMT2B & rs3848662 & 1f & KMT2B \\
\hline rs9561489 & 13 & NS & NS & GPC6 & rs9556347 & $3 a$ & GPC6 \\
\hline rs4934956 & 10 & 5 & NS & СICP9-ABCD1P2 & . & . & . \\
\hline rs9897342 & 17 & $2 b$ & 0.5 & MYO18A-TWF1P1 & rs7225149 & $2 a$ & MYO18A \\
\hline rs756134 & 1 & 4 & NS & $A L X 3-U B L 4 B$ & & & . \\
\hline rs4926465 & 1 & 6 & NS & KIF28P & rs7520180 & $3 a$ & KIF28P \\
\hline rs552976 & 2 & $3 a$ & 0.27 & $A B C B 11$ & & & . \\
\hline rs563694 & 2 & NS & 0.1 & G6PC2-ABCB11 & rs566879 & $3 a$ & $A B C B 11$ \\
\hline rs1529186 & 3 & 4 & 0.1 & $\begin{array}{c}\text { LOC100422385- } \\
\text { LOC440973 }\end{array}$ & rs62281690 & $3 a$ & $\begin{array}{c}\text { LOC100422385- } \\
\text { LOC440973 }\end{array}$ \\
\hline rs11049786 & 12 & 6 & NS & CCDC91-FAR2 & . & . & . \\
\hline rs9510793 & 13 & 6 & NS & TNFRSF19 & . & . & . \\
\hline rs61748746* & 4 & 6 & NS & N4BP2 & . & . & . \\
\hline rs2874641 & 17 & 5 & NS & SHISA6 & . & . & . \\
\hline rs560887 & 2 & NS & NS & G6PC2 & . & . & . \\
\hline rs7025331 & 9 & 4 & NS & $\begin{array}{c}\text { SMARCA2- } \\
\text { LOC102723835 }\end{array}$ & . & & . \\
\hline rs7917910 & 10 & NS & NS & C10orf90-DOCK1 & . & . & . \\
\hline rs349707 & 3 & 6 & NS & SLC6A1-HRH1 & . & . & . \\
\hline rs61748749* & 4 & $2 b$ & NS & N4BP2 & . & . & . \\
\hline rs9873177 & 3 & 5 & 0.1 & PDIA5 & . & . & . \\
\hline rs739924 & 17 & 1f & 1 & SPATA20 & rs9913430 & $1 \mathrm{a}$ & SPATA20 \\
\hline rs 12505859 & 4 & NS & 0.5 & ICE2P1-LINC01060 & . & & . \\
\hline
\end{tabular}


Appendix Table 6 continued....

\begin{tabular}{|c|c|c|c|c|c|c|c|}
\hline SNP & Chr & $\begin{array}{c}\text { RegulomeDB } \\
\text { score }^{\dagger}\end{array}$ & F-SNP ${ }^{* *}$ & Gene & $\begin{array}{l}\text { Correlated } \\
\text { SNP }\end{array}$ & $\begin{array}{c}\text { RegulomeDB } \\
\text { score }^{\dagger}\end{array}$ & $\begin{array}{c}\text { Gene } \\
\text { (correlated SNPs) }\end{array}$ \\
\hline rs 7322477 & 13 & $\mathrm{NS}$ & 0 & LINC00284 & rs4418956 & $2 \mathrm{~b}$ & LINC00284 \\
\hline rs1978759 & 10 & 4 & NS & MGMT & rs1978756 & $2 b$ & MGMT \\
\hline rs 725038 & 17 & 6 & 0.21 & ACACA & . & . & . \\
\hline rs729867 & 2 & 6 & 0.1 & CSRNP3 & . & . & . \\
\hline rs4875742 & 8 & 6 & NS & CSMD1 & . & & . \\
\hline rs 1720326 & 11 & 4 & NS & SCN3B-ZNF202 & rs1275051 & 1f & SCN3B-ZNF202 \\
\hline rs1713676 & 11 & 5 & NS & LOC390251-USP28 & rs11214739 & $3 a$ & USP28 \\
\hline rs3857193 & 4 & $3 a$ & NS & SORCS2 & & & . \\
\hline rs6987111 & 8 & 6 & NS & CCDC25 & rs9314355 & 1d & $\begin{array}{c}\text { LOC100130612- } \\
\text { SCARA5 }\end{array}$ \\
\hline rs6987111 & 8 & 6 & NS & CCDC25 & rs17057911 & 1f & $P B K$ \\
\hline rs6987111 & 8 & 6 & NS & CCDC25 & rs17057863 & 1f & ESCO2 \\
\hline rs4801931 & 19 & 5 & NS & ZNF611 & 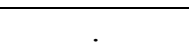 & & . \\
\hline rs12940887 & 17 & NS & NS & ZNF652 & rs16948048 & $3 a$ & ZNF652 \\
\hline rs7225787 & 17 & NS & NS & ZNF652 & & & . \\
\hline rs11079867 & 17 & NS & NS & FLJ40194-ZNF652 & rs2011767 & $2 a$ & FLJ40194-ZNF652 \\
\hline rs4680582 & 3 & 6 & 0.1 & SMC4 & rs73155903 & $2 b$ & TRIM59 \\
\hline rs1429143 & 6 & NS & NS & ELOVL5-NANOGP3 & & & . \\
\hline rs 10506041 & 12 & NS & NS & LOC101928705-FAR2 & . & . & . \\
\hline rs1915337 & 3 & NS & NS & $\begin{array}{l}\text { LOC100422385- } \\
\text { LOC440973 }\end{array}$ & . & . & . \\
\hline rs6962352 & 7 & 5 & 0.18 & HIP1 & . & . & . \\
\hline rs634413 & 13 & 5 & NS & LINC00544 & rs4769779 & $3 a$ & LINC00544 \\
\hline rs4074605 & 17 & 4 & NS & SYNRG & rs7224979 & 1d & SYNRG \\
\hline
\end{tabular}


Appendix Table 6 continued....

\begin{tabular}{|c|c|c|c|c|c|c|c|}
\hline SNP & Chr & $\begin{array}{c}\text { RegulomeDB } \\
\text { score }^{\dagger}\end{array}$ & F-SNP** & Gene & $\begin{array}{l}\text { Correlated } \\
\text { SNP }\end{array}$ & $\begin{array}{c}\text { RegulomeDB } \\
\text { score }^{\dagger}\end{array}$ & $\begin{array}{c}\text { Gene } \\
\text { (correlated SNPs) }\end{array}$ \\
\hline rs4074605 & 17 & 4 & NS & SYNRG & rs3110626 & $1 \mathrm{f}$ & SYNRG \\
\hline rs4074605 & 17 & 4 & NS & SYNRG & rs6607279 & $1 \mathrm{f}$ & SYNRG \\
\hline rs 4074605 & 17 & 4 & NS & SYNRG & rs8080313 & $1 \mathrm{f}$ & SYNRG \\
\hline rs 7407281 & 18 & NS & NS & $\begin{array}{c}\text { LOC100128022- } \\
\text { NFE2L3P1 }\end{array}$ & & & . \\
\hline rs 10778311 & 12 & 5 & NS & TXNRD1 & rs7138523 & $3 a$ & TXNRD1 \\
\hline rs10778311 & 12 & 5 & NS & TXNRD1 & rs4329742 & $3 a$ & TXNRD1 \\
\hline rs 10778311 & 12 & 5 & NS & TXNRD1 & rs4569072 & $3 a$ & TXNRD1 \\
\hline rs7492685 & 14 & 6 & NS & RPL18P1-ATP5GP2 & . & . & . \\
\hline rs4246284 & 15 & 6 & NS & $\begin{array}{c}\text { RPL31P55- } \\
\text { FAM149B1P1 }\end{array}$ & rs 12898262 & $3 a$ & $\begin{array}{c}\text { RPL31P55- } \\
\text { FAM149B1P1 }\end{array}$ \\
\hline rs 10128580 & 11 & 5 & 0 & ZBTB16 & . & 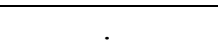 & . \\
\hline rs 7243843 & 18 & 5 & 0.55 & DLGAP1 & rs 7228679 & $2 b$ & DLGAP1 \\
\hline rs1602491 & 4 & 5 & 0.1 & $\begin{array}{c}\text { ADAM29- } \\
\text { LOC100129957 }\end{array}$ & . & . & . \\
\hline rs17439813 & 12 & NS & NS & LOC101928705- FAR2 & ㅇ & . & . \\
\hline rs9373051 & 6 & 6 & NS & EYA4 & . & . & . \\
\hline rs309026 & 12 & 5 & NS & LOC101928705- FAR2 & & & . \\
\hline rs 11011225 & 10 & 5 & NS & $\begin{array}{c}\text { TMEM161BP1- } \\
\text { TACC1P1 }\end{array}$ & & & . \\
\hline rs12904345 & 15 & NS & NS & $\begin{array}{c}\text { RPL31P55- } \\
\text { FAM149B1P1 }\end{array}$ & rs12898262 & $3 a$ & $\begin{array}{c}\text { RPL31P55- } \\
\text { FAM149B1P1 }\end{array}$ \\
\hline rs 7700370 & 5 & 6 & 0.1 & ADAMTS12 & . & &. \\
\hline rs910850 & 20 & 5 & NS & DOK5 & & & . \\
\hline rs 12822012 & 12 & NS & NS & RPL13AP21- SLC38A1 & . & & . \\
\hline rs7237664 & 18 & 5 & NS & CDH2-ARIH2P & & & . \\
\hline
\end{tabular}


Appendix Table 6 continued....

\begin{tabular}{|c|c|c|c|c|c|c|c|}
\hline SNP & Chr & $\begin{array}{c}\text { RegulomeDB } \\
\text { score }^{\dagger}\end{array}$ & F-SNP $^{* *}$ & Gene & $\begin{array}{c}\text { Correlated } \\
\text { SNP }\end{array}$ & $\begin{array}{c}\text { RegulomeDB } \\
\text { score }^{\dagger}\end{array}$ & $\begin{array}{c}\text { Gene } \\
\text { (correlated SNPs) }^{\text {(n) }}\end{array}$ \\
\hline rs1821567 & 8 & NS & NS & C8orf34 & $\cdot$ & $\cdot$ & $\cdot$ \\
\hline rs1477656 & 5 & 6 & $\mathbf{0 . 2 4}$ & $\begin{array}{c}\text { LOC285706- } \\
\text { EEF1A1P20 }\end{array}$ & $\cdot$ & $\cdot$ & $\cdot$ \\
\hline rs7163190 & 15 & NS & NS & GOLGA8B-GJD2 & $\cdot$ & $\cdot$ & $\cdot$ \\
\hline rs11648233 & 16 & 6 & $\mathbf{0 . 5}$ & HSD17B2 & $\cdot$ & $\cdot$ & $\cdot$ \\
\hline
\end{tabular}

Note: Some associated SNPs showed multiple statistically correlated functional variants

$\mathrm{Chr}=$ Chromosome; $\mathrm{NS}=$ No score; $\mathrm{SNP}=$ single nucleotide polymorphism; $*$ Missense; ${ }^{\dagger}$ SNPs change or create a binding site for transcription factors

${ }^{\dagger}$ RegulomDb predicts score from 1 to 7 . A score of one suggests SNPs have higher functionality and may play an important role in gene regulation A score of 7 predicts least functionality

${ }^{* *}$ F-SNP predicts score from 0 to 1 . A score of one suggest higher functionality and a score zero suggests no functional role of a SNP 
Appendix Table 7: Bioinformatic analysis of genetic risk markers for the top one hundred gender-specific associations with childhood ALL with the highest effect sizes $(P<0.05)$

\begin{tabular}{|c|c|c|c|c|c|c|c|c|}
\hline SNP & Chr & Feature & $\begin{array}{c}\text { RegulomeDB } \\
\text { Score }^{\dagger}\end{array}$ & F-SNP ${ }^{* *}$ & Gene/region & $\begin{array}{l}\text { Correlated } \\
\text { SNP }\end{array}$ & $\underset{\text { score }^{\dagger}}{\text { RegulomeDB }}$ & $\begin{array}{c}\text { Gene } \\
\text { (Correlated SNPs) }\end{array}$ \\
\hline rs12722042* & 6 & coding & 4 & 0.75 & HLA-DQA1 & rs28619112 & $3 \mathrm{a}$ & HLA-DQA1 \\
\hline rs $12722039^{*}$ & 6 & coding & 4 & 0.41 & HLA-DQA1 & rs28619112 & $3 a$ & HLA-DQA1 \\
\hline rs11992342 & 8 & intron & 6 & 0.5 & ADAM28 & . & . & . \\
\hline rs80040922 & 21 & coding & NS & NS & UMODL & . & . & . \\
\hline rs61753605 & 6 & coding & 5 & NS & LOC100996481 & . & . & . \\
\hline rs $35665085^{*}$ & 22 & coding & 6 & 0 & CECR5 & . & . & . \\
\hline rs143021649 & 3 & coding & NS & NS & CNTN3 & . & . & . \\
\hline rs6795524 & 3 & intron & 5 & 0.26 & PROS1 & rs76514818 & $3 a$ & $A R L 13 B$ \\
\hline rs10003468 & 4 & intergenic & NS & 0.1 & FAT4-NUPL1P1 & . & . & . \\
\hline rs10131142 & 14 & intergenic & NS & 0.5 & EDDMЗА-EDDMЗВ & . & . & . \\
\hline rs3732042 & 2 & 3utr & 6 & 0.24 & CNRIP1 & rs73935338 & $2 \mathrm{~b}$ & PPP3R1- CNRIP1 \\
\hline rs34022679 & 4 & coding & 5 & 0.1 & GRK4 & . & . & . \\
\hline rs $7829965^{*}$ & 8 & coding & $2 \mathrm{~b}$ & 0.5 & ADAM28 & . & . & . \\
\hline rs7097067 & 10 & intergenic & 6 & 0.5 & PPA1-NPFFR1 & rs60660561 & $2 b$ & PPA1 \\
\hline rs7097067 & 10 & intergenic & 6 & 0.5 & PPA1-NPFFR1 & rs56938544 & $2 b$ & NPFFR1 \\
\hline rs35912436 & 7 & intergenic & 6 & NS & TMEM106B-VWDE & rs 2192840 & $2 b$ & TMEM106B-VWDE \\
\hline rs2160271 & 7 & intergenic & $\mathrm{NS}$ & NS & TMEM106B-VWDE & rs2192840 & $2 b$ & TMEM106B-VWDE \\
\hline rs17588454 & 13 & intergenic & 5 & NS & $\begin{array}{l}\text { LINC00544- } \\
\text { LINC00384 }\end{array}$ & rs 4534683 & $2 b$ & $\begin{array}{l}\text { LINC00544- } \\
\text { LINC00384 }\end{array}$ \\
\hline rs368390 & 18 & intron & 6 & 0.5 & C18orf42 & . & . &. \\
\hline rs $17080410^{*}$ & 6 & coding & 5 & 0 & PLEKHG1 & . & . & . \\
\hline rs61742396 & 6 & coding & 5 & NS & PLEKHG1 & rs57264696 & $2 \mathrm{a}$ & $\begin{array}{l}\text { PLEKHG1- } \\
\text { MTHFD1L }\end{array}$ \\
\hline
\end{tabular}


Appendix Table 7 continued....

\begin{tabular}{|c|c|c|c|c|c|c|c|c|}
\hline SNP & Chr & Feature & $\begin{array}{c}\text { RegulomeDB } \\
\text { Score }^{\dagger} \\
\end{array}$ & F-SNP ${ }^{* *}$ & Gene/region & $\begin{array}{c}\text { Correlated } \\
\text { SNP } \\
\end{array}$ & $\begin{array}{c}\begin{array}{c}\text { RegulomeDB } \\
\text { score }^{\dagger}\end{array} \\
\end{array}$ & $\begin{array}{c}\text { Gene } \\
\text { (Correlated SNPs) } \\
\end{array}$ \\
\hline rs35672330 & 1 & coding & 5 & 0 & EXO5 & 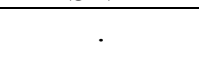 & . & . \\
\hline rs 1503292 & 4 & intron & 6 & NS & TLL1 & . & . & . \\
\hline rs 1978526 & 2 & intergenic & 6 & NS & KCNH7- RPL7P61 & rs72623141 & $3 a$ & KCNH7-RPL7P61 \\
\hline rs6887604 & 5 & intergenic & 6 & NS & $\begin{array}{c}\text { CD180- } \\
\text { LOC101928819 }\end{array}$ & rs78308574 & $3 a$ & $\begin{array}{c}\text { CD180- } \\
\text { LOC101928819 }\end{array}$ \\
\hline rs 10063286 & 5 & intergenic & NS & 0.1 & DRD1-SFXN1 & rs201488474 & $3 a$ & DRD1-SFXN1 \\
\hline rs16879126 & 4 & intron & 4 & NS & NT5C3 & rs 72555715 & $2 \mathrm{c}$ & NT5C3A \\
\hline rs34265667 & 8 & coding & 5 & 0.14 & ANK1 & . & . & . \\
\hline rs $34664882^{*}$ & 8 & coding & 5 & 0 & ANK1 & • & . & . \\
\hline rs61731389* & 10 & coding & 6 & NS & PCDH15 & rs 72797047 & $3 a$ & PCDH15 \\
\hline rs4764332 & 12 & intergenic & NS & NS & $\begin{array}{l}\text { GABARAPL1- } \\
\text { KLRD1 }\end{array}$ & rs73060081 & $2 b$ & $\begin{array}{c}\text { GABARAPL1- } \\
\text { KLRD1 }\end{array}$ \\
\hline rs4496864 & 7 & intergenic & NS & NS & TMEM106B-VWDE & rs2192840 & $2 b$ & TMEM106B-VWDE \\
\hline rs6936494 & 6 & intergenic & NS & NS & $\begin{array}{c}\text { ACTBP8- } \\
\text { LOC101928936 }\end{array}$ & . & . & . \\
\hline rs3847262 & 9 & coding & NS & 0.87 & TPD52L3 & rs10758768 & $2 a$ & TPD52L3-UHRF2 \\
\hline rs2890707 & 9 & 3utr & $2 \mathbf{b}$ & NS & TPD52L3 & rs10758768 & $2 a$ & TPD52L3-UHRF2 \\
\hline rs7626907 & 3 & intergenic & NS & NS & KCNH8-EFHB & . & . & . \\
\hline rs2229528 & 3 & coding & $\mathbf{2 b}$ & NS & ACAA1 & . & . & . \\
\hline rs9842465 & 3 & intron & NS & 0.24 & SCHIP1 & rs 13434009 & $3 a$ & SCHIP1 \\
\hline rs6796558 & 3 & intergenic & 6 & 0.1 & PYDC2-FGF12 & . & . & . \\
\hline rs55882716 & 5 & coding & 5 & NS & MAST4 & . & . & . \\
\hline rs 1030270 & 5 & intron & NS & NS & CYFIP2 & rs61088678 & $3 a$ & CYFIP2 \\
\hline rs205944 & 6 & intron & NS & NS & SLC35F1 & . & . & . \\
\hline rs381783 & 6 & intron & NS & NS & IL20RA & rs276497 & $2 b$ & IL20RA \\
\hline
\end{tabular}


Appendix Table 7 continued....

\begin{tabular}{|c|c|c|c|c|c|c|c|c|}
\hline SNP & Chr & Feature & $\begin{array}{c}\text { RegulomeDB } \\
\text { Score }^{\dagger}\end{array}$ & F-SNP ${ }^{* *}$ & Gene/region & $\begin{array}{c}\text { Correlated } \\
\text { SNP }\end{array}$ & $\begin{array}{c}\text { RegulomeDB } \\
\text { score }^{\dagger}\end{array}$ & $\begin{array}{c}\text { Gene } \\
\text { (Correlated SNPs) }\end{array}$ \\
\hline rs381783 & 6 & intron & $\mathrm{NS}$ & NS & IL20RA & rs276498 & $2 b$ & IL20RA \\
\hline rs381783 & 6 & intron & NS & NS & IL20RA & rs276499 & $2 b$ & IL20RA \\
\hline rs11980237 & 7 & intron & NS & 0 & KDELR2 & . & . & . \\
\hline rs 720872 & 7 & intergenic & NS & NS & $D G K B-A G M O$ & rs218078 & $3 a$ & DGKB-AGMO \\
\hline rs $147596194^{*}$ & 9 & intron & 4 & NS & RGS3 & rs118167986 & $2 b$ & RGS3 \\
\hline rs $147596194^{*}$ & 9 & intron & 4 & NS & RGS3 & rs76385691 & $2 b$ & RGS3 \\
\hline rs147596194* & 9 & intron & 4 & NS & RGS3 & rs117507747 & $2 b$ & RGS3 \\
\hline rs147596194* & 9 & intron & 4 & NS & RGS3 & rs113069800 & $2 b$ & RGS3 \\
\hline rs $147596194^{*}$ & 9 & intron & 4 & NS & RGS3 & rs 150767530 & $2 b$ & $\begin{array}{c}\text { RGS3- } \\
\text { LOC100288542 }\end{array}$ \\
\hline rs61745456 & 9 & coding & 5 & NS & MAMDC4 & . & . & . \\
\hline rs117923468 & 19 & coding & NS & NS & WDR87 & . & . & . \\
\hline rs $182319278^{*}$ & 19 & coding & NS & NS & WDR87 & . & . & . \\
\hline rs9305777 & 5 & intergenic & 5 & NS & MIR99AHG-NEK4P1 & . & . & . \\
\hline rs8129749 & 21 & intergenic & NS & NS & $\begin{array}{c}\text { LOC339622- } \\
\text { RNA5SP489 }\end{array}$ & rs11909912 & $3 a$ & $\begin{array}{c}\text { LOC339622- } \\
\text { RNA5SP489 }\end{array}$ \\
\hline rs985456 & 21 & intergenic & 5 & NS & $\begin{array}{c}\text { MARCKSP1- } \\
\text { LOC100996571 }\end{array}$ & rs 79015379 & $3 a$ & $\begin{array}{c}\text { LOC102724317- } \\
\text { CYYR1-AS1 }\end{array}$ \\
\hline rs16863233 & 3 & intron & NS & 0.24 & $L P P$ & . & . & $\cdot$ \\
\hline rs 7037100 & 9 & intron & 5 & NS & ZNF782 & rs12349952 & 1f & ZNF510 \\
\hline rs 7630157 & 3 & intron & NS & 0.1 & EGFEM1P & . & . & . \\
\hline rs62640905 & 16 & coding & 5 & NS & OSGIN1 & . & . & . \\
\hline rs $16973890^{*}$ & 19 & intron & 4 & 0.62 & ZNF571 & . & . & . \\
\hline rs35693261 & 20 & coding & 5 & 0.1 & MTG2 & . & . & . \\
\hline rs7539097 & 1 & intron & 5 & NS & $T N R$ & rs80216135 & $3 a$ & $T N R$ \\
\hline
\end{tabular}


Appendix Table 7 continued....

\begin{tabular}{|c|c|c|c|c|c|c|c|c|}
\hline SNP & Chr & Feature & $\begin{array}{c}\text { RegulomeDB } \\
\text { Score }^{\dagger}\end{array}$ & F-SNP $P^{* *}$ & Gene/region & $\begin{array}{l}\text { Correlated } \\
\text { SNP }\end{array}$ & $\begin{array}{c}\text { RegulomeDB } \\
\text { score }^{\dagger}\end{array}$ & $\begin{array}{c}\text { Gene } \\
\text { (Correlated SNPs) }\end{array}$ \\
\hline rs9493135 & 6 & intergenic & 4 & NS & ENPP1-CTGF & rs73779142 & $3 a$ & ENPP1-CTGF \\
\hline rs9493135 & 6 & intergenic & 4 & $\mathrm{NS}$ & ENPP1-CTGF & rs9493137 & $3 a$ & ENPP1-CTGF \\
\hline rs9493135 & 6 & intergenic & 4 & NS & ENPP1-CTGF & rs57896928 & $3 a$ & ENPP1-CTGF \\
\hline rs6722598 & 2 & 5upstream & 5 & 0.24 & MFF & rs138676164 & $3 a$ & LOC654841 \\
\hline rs6722598 & 2 & 5upstream & 5 & 0.24 & MFF & rs201829089 & $3 a$ & LOC654841 \\
\hline rs1049894 & 2 & coding & NS & 0.37 & PSME4 & rs10180492 & $3 a$ & GPR75-ASB3 \\
\hline rs1049894 & 2 & coding & NS & 0.37 & PSME4 & rs78542544 & $3 a$ & PSME4 \\
\hline rs11904365 & 2 & intergenic & 6 & 0.1 & PSME4-RPL21P30 & . & . & . \\
\hline rs 10003958 & 4 & intron & 5 & 0.1 & $R A B 28$ & rs7673680 & $3 b$ & $R A B 28$ \\
\hline rs9993832 & 4 & intron & 5 & NS & $R A B 28$ & rs 7673680 & $3 \mathrm{~b}$ & $R A B 28$ \\
\hline rs36041430 & 8 & coding & $\mathrm{NS}$ & 0 & ADAM28 & . & . & . \\
\hline rs $78888579^{*}$ & 1 & coding & 5 & NS & CROCC & rs116314250 & $2 \mathrm{~b}$ & CROCC \\
\hline rs $78888579^{*}$ & 1 & coding & 5 & $\mathrm{NS}$ & CROCC & rs140067167 & $2 b$ & CROCC \\
\hline rs 10218650 & 1 & intergenic & NS & 0.1 & CDC73-KCNT2 & . & . & . \\
\hline rs 13405142 & 2 & intergenic & NS & 0.1 & $\begin{array}{c}\text { HNRNPA1P57- } \\
\text { LDHAP3 }\end{array}$ & . & . & . \\
\hline rs10194197 & 2 & intergenic & 6 & 0.1 & GCFC2- SUCLA2P2 & . & . & . \\
\hline rs61741435* & 2 & coding & 6 & NS & CCDC173 & rs79306213 & $2 \mathrm{~b}$ & $P P I G$ \\
\hline rs4856270 & 3 & intron & 5 & 0.1 & CADM2 & rs6809486 & $3 a$ & CADM2 \\
\hline rs2165084 & 4 & intergenic & $\mathbf{3 a}$ & 0.27 & KIAA0922-WDR45P & rs2118864 & $2 a$ & KIAA0922-WDR45P \\
\hline rs7703414 & 5 & intergenic & 6 & 0.1 & INTS6P1-LINC00603 & . & . & . \\
\hline rs11751765* & 6 & coding & 5 & 0.9 & TRERF1 & . & . & . \\
\hline rs7797334 & 7 & intergenic & 5 & NS & $\begin{array}{c}\text { LOC101927558- } \\
\text { ISPD }\end{array}$ & . & & . \\
\hline
\end{tabular}


Appendix Table 7 continued....

\begin{tabular}{|c|c|c|c|c|c|c|c|c|}
\hline SNP & Chr & Feature & $\begin{array}{c}\text { RegulomeDB } \\
\text { Score }^{\dagger} \\
\end{array}$ & F-SNP ${ }^{* *}$ & Gene/region & $\begin{array}{c}\text { Correlated } \\
\text { SNP } \\
\end{array}$ & $\begin{array}{c}\begin{array}{c}\text { RegulomeDB } \\
\text { score }^{\dagger}\end{array} \\
\end{array}$ & $\begin{array}{c}\text { Gene } \\
\text { (Correlated SNPs) }\end{array}$ \\
\hline rs4278110 & 7 & intergenic & 5 & NS & $\begin{array}{c}\text { LOC101927558- } \\
\text { ISPD }\end{array}$ & rs6583519 & $3 a$ & $\begin{array}{c}C D C 14 C- \\
L O C 100533714\end{array}$ \\
\hline rs11987106 & 8 & intron & 6 & NS & COLEC10 & . & . & $\cdot$ \\
\hline rs61735202 & 9 & coding & 6 & NS & CCIN & . & . & . \\
\hline rs943811 & 10 & intergenic & 5 & NS & LINC01163-MGMT & . & . & . \\
\hline rs10430621 & 10 & intergenic & 6 & NS & TCERG1L-PPP2R2D & . & . & . \\
\hline rs11182332 & 12 & intron & 6 & NS & TMEM117 & . & . & . \\
\hline rs2293624* & 12 & coding & NS & 0.37 & C12orf73 & . & . & . \\
\hline rs $4770684^{*}$ & 13 & coding & NS & 0.32 & PARP4 & . & . & . \\
\hline rs7182827 & 15 & intron & 6 & NS & $U B E 3 A$ & rs74946267 & $2 b$ & $U B E 3 A$ \\
\hline rs 12621721 & 2 & intron & 6 & 0.1 & MAPЗK19/YSK4 & rs78265845 & $2 b$ & MAPЗК19 \\
\hline rs7275217 & 21 & intergenic & 6 & NS & KRTAP21-1-KRTAP8 & . & . & . \\
\hline rs41299607 & 1 & coding & 6 & 0.1 & RGSL1 & . & . & . \\
\hline rs9282777 & 11 & coding & 5 & 0.37 & LMO2 & . & . & . \\
\hline rs10520291 & 2 & intergenic & NS & 0.1 & CTNNA2-SUCLG1 & . & . & . \\
\hline rs12037891 & 1 & intron & 6 & 0.1 & $P D E 4 B$ & . & . & . \\
\hline rs73022563* & 5 & coding & 5 & NS & PLEKHG4B & . & . & . \\
\hline rs $130069^{*}$ & 6 & coding & 5 & 1 & CCHCR1 & . & . & . \\
\hline rs6929434 & 6 & 5upstream & $1 \mathrm{~b}$ & 0.5 & TCF19 & . & . & . \\
\hline rs10975823 & 9 & intron & $\mathrm{NS}$ & 0 & $K D M 4 C$ & rs10118219 & $2 \mathrm{a}$ & $K D M 4 C$ \\
\hline rs4645656 & 9 & coding & 5 & 0.5 & ZNF782 & rs12349952 & $1 \mathrm{f}$ & ZNF510 \\
\hline rs7848710 & 9 & intron & NS & NS & ZNF782 & rs12349952 & 1f & ZNF510 \\
\hline rs6477733 & 9 & intron & 5 & 0.5 & ZNF782 & rs12349952 & $1 \mathrm{f}$ & ZNF510 \\
\hline
\end{tabular}


Appendix Table 7 continued....

\begin{tabular}{|c|c|c|c|c|c|c|c|c|}
\hline SNP & Chr & Feature & $\begin{array}{c}\text { RegulomeDB } \\
\text { Score }^{\dagger} \\
\end{array}$ & F-SNP $^{* *}$ & Gene/region & $\begin{array}{c}\text { Correlated } \\
\text { SNP }\end{array}$ & $\begin{array}{c}\begin{array}{c}\text { RegulomeDB } \\
\text { score }^{\dagger}\end{array} \\
\end{array}$ & $\begin{array}{c}\text { Gene } \\
\text { (Correlated SNPs) }\end{array}$ \\
\hline rs17000817 & 9 & intergenic & 5 & 0.5 & $\begin{array}{c}\text { RNA5SP489- } \\
\text { RPL13AP7 }\end{array}$ & 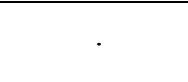 & 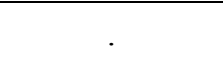 & 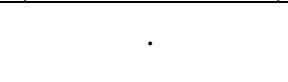 \\
\hline rs2633820 & 3 & intron & NS & 0.1 & SUMF1 & . & . & . \\
\hline rs9469223 & 6 & intergenic & NS & NS & NEDD9-HIVEP1 & rs17608111 & $3 a$ & NEDD9-HIVEP1 \\
\hline
\end{tabular}

Note: Some associated SNPs showed multiple statistically correlated functional variants

$\mathrm{Chr}=$ Chromosome; NS = No score; SNP = single nucleotide polymorphisms; $*$ Missense; ${ }^{\dagger}$ SNPs change or create a binding site for transcription factors

${ }^{\dagger}$ RegulomDb predicts score from 1 to 7 . A score of one suggests SNPs have higher functionality and may play an important role in gene regulation. A score of 7 predicts least functionality

${ }^{* *}$ F-SNP predicts score from 0 to 1 . A score of one suggest higher functionality and a score zero suggests no functional role of a SNP 
Appendix Table 8: Expression quantitative trait locus analysis of gender-specific associated genetic risk variants

\begin{tabular}{|c|c|c|c|c|}
\hline SNP & Chr & Feature & Gene & eQTL \\
\hline rs7912381* & 2 & intergenic & $\begin{array}{l}\text { TCERG1L- } \\
\text { PPP2R2D }\end{array}$ & FGD6 \\
\hline rs798292* & 7 & intronic & MAGI2 & MYST2, ATAD2B, EIF1AX \\
\hline rs $1550017^{*}$ & 4 & intergenic & GRIA2-LOC340017 & FGD6 \\
\hline rs12054636 ${ }^{*}$ & 4 & intergenic & GRIA2-LOC340017 & FGD6 \\
\hline rs $560887^{*}$ & 2 & intronic & G6PC2 & P4HA2 \\
\hline rs739924* & 17 & intronic & SPATA20 & SPATA20 \\
\hline rs $725038^{*}$ & 17 & intronic & ACACA & COL9A3 \\
\hline rs $12904345^{*}$ & 15 & intergenic & $\begin{array}{c}\text { RPL31P55- } \\
\text { FAM149B1P1 }\end{array}$ & $R O P N 1, R O P N 1 B$ \\
\hline rs4299194* & 17 & intronic & $A C A C A$ & $\begin{array}{c}\text { LOC100131190, MX2, } \\
\text { PLSCR1 }\end{array}$ \\
\hline rs $12461333^{*}$ & 19 & intergenic & UPK1A-ZBTB32 & CANT1, CLPTM1, \\
\hline rs9913430* & 17 & intronic & SPATA20 & SPATA20 \\
\hline rs9493135 & 6 & intergenic & ENPP1-CTGF & $\begin{array}{c}\text { NPAL2, ENOSF1, SMG1, } \\
\text { PTPN11, ACACA, GALK2, } \\
\text { LOC440354, LOC595101, } \\
\text { LOC641298 }\end{array}$ \\
\hline
\end{tabular}

$\mathrm{eQTL}=$ expression quantitative trait loci; $\mathrm{Chr}=$ chromosome

${ }^{*}$ Gender-specific results based on the P value; **Gender-specific results based on the effect sizes 
Appendix Table 9: Gender- and age-specific single nucleotides polymorphisms and their previous GWAS associations

\begin{tabular}{|c|c|c|c|c|}
\hline SNP & Gene & Disease/trait & Results & PUBMED ID \\
\hline rs552976 & G6PC2 & Glycated hemoglobin levels & Gender P value & 20858683 \\
\hline rs563694 & G6PC2 & Fasting plasma glucose & Gender P value & 18521185 \\
\hline rs560887 & G6PC2 & Glycemic traits (pregnancy) & Gender $\mathrm{P}$ value & 23903356 \\
\hline rs560887 & G6PC2 & Metabolite levels & Gender P value & 22916037 \\
\hline rs560887 & G6PC2 & Fasting glucose-related traits (interaction with BMI) & Gender P value & 22581228 \\
\hline rs560887 & G6PC2 & Fasting plasma glucose & Gender P value & 22508271 \\
\hline rs560887 & G6PC2 & Metabolic syndrome & Gender P value & 22399527 \\
\hline rs560887 & G6PC2 & Metabolite levels & Gender P value & 22286219 \\
\hline rs560887 & G6PC2 & Fasting glucose-related traits & Gender P value & 20081858 \\
\hline rs560887 & G6PC2 & Fasting glucose-related traits & Gender P value & 20081858 \\
\hline rs560887 & G6PC2 & Metabolic traits & Gender $P$ value & 19060910 \\
\hline rs560887 & G6PC2 & Fasting plasma glucose & Gender P value & 19060907 \\
\hline rs560887 & G6PC2 & Fasting plasma glucose & Gender P value & 18451265 \\
\hline rs12940887 & ZNF652 & Blood pressure & Gender P value & 21909110 \\
\hline rs9305777 & intergenic & Platelet aggregation & Gender OR & 17903294 \\
\hline rs5028798 & EHF & Volumetric brain MRI & Age $P$ value & 17903297 \\
\hline rs4948088 & COBL & Type 1 diabetes & Age $P$ value & 19430480 \\
\hline rs2116078 & KCNB2 & Multiple sclerosis (age of onset) & Age $P$ value & 19010793 \\
\hline rs 12188300 & IL12B & Psoriatic arthritis & Age OR & 20953186 \\
\hline rs28421666 & intergenic & Nasopharyngeal carcinoma & Age OR & 20512145 \\
\hline rs7169431 & intergenic & Chronic lymphocytic leukemia & Age OR & 23770605 \\
\hline rs7169431 & intergenic & Chronic lymphocytic leukemia & Age OR & 20062064 \\
\hline
\end{tabular}

SNP = single nucleotide polymorphism; OR: odds ratio 


\section{Appendix Table 10: Genes showing gender-specific associations (ranked by their $P$ values) and their interactions}

with chemicals in studies curated in the Comparative Toxicological Database

\begin{tabular}{|c|c|c|c|}
\hline Gene/region & SNP & Chemical interaction & PubMed IDs \\
\hline RASSF2 & rs4813720 & $\begin{array}{l}\text { aflatoxin B1, arsenite, benzene, bisphenol A, } \\
\text { copper sulfate, dust, ethinyl estradiol, } \\
\text { tetrachlorodibenzodioxin, 4-hydroxy-2-nonenal, } \\
\text { acetaminophen }\end{array}$ & $\begin{array}{l}22100608,18929588,19162166, \\
12075121,12075121,20080153, \\
20436886,17562736,19549813, \\
17805423\end{array}$ \\
\hline TCERG1L & $\begin{array}{l}\text { rs } 7912381 * \\
\text { rs } 12219278\end{array}$ & $\begin{array}{l}\text { arsenite, fonofos, parathion, terbufos, } \\
\text { pentachlorophenol }\end{array}$ & $\begin{array}{c}23974009,22847954,22847954, \\
23724009,23892564\end{array}$ \\
\hline$P P P 2 R 2 D$ & rs7912381* & $\begin{array}{l}\text { benzo(a)pyrene diol epoxide, PCBs, } \\
\text { acetaminophen, bisphenol A, } \\
\text { tetrachlorodibenzodioxin, 2,6-dinitrotoluene, } \\
\text { sodium selenite }\end{array}$ & $\begin{array}{c}20971185,19114083,17562736, \\
24534842,21570461,21346803, \\
18175754\end{array}$ \\
\hline MAGI2 & rs798292 & $\begin{array}{l}\text { acetaminophen, aflatoxin B1, arsenite, } \\
\text { benzo(a)pyrene, benzo(a)pyrene diol epoxide, } \\
\text { bisphenol A, PCB, methoxychlor, } \\
\text { tetrachlorodibenzodioxin, tungsten carbide, } \\
\text { ammonium chloride, bisphenol A, dibutyl phthalate, } \\
\text { diethylhexyl phthalate, ammonium chloride }\end{array}$ & $\begin{array}{l}22230336,21632981,22100608, \\
23974009,20106945,20382639, \\
21632981,21871943,23359474, \\
23303685,21177773,24935251, \\
20105288,23829299,19114083, \\
23829299,16483693,23359474, \\
16483693 \\
\end{array}$ \\
\hline SLIT2 & $\begin{array}{l}\text { rs } 13107783^{*} \\
\text { rs4470624* }\end{array}$ & $\begin{array}{l}\text { aflatoxin B1, asbestos, atrazine, benzo(a)pyrene, } \\
\text { benzo(a)pyrene diol epoxide, estradiol, bisphenol } \\
\text { A, estrogens, N-acetyl-4-benzoquinoneimine, } \\
\text { tetrachlorodibenzodioxin, chlorpyrifos, dibutyl } \\
\text { phthalate, flusilazole, hexaconazole, nitrofen, } \\
\text { thiram, 2,6-dinitrotoluene, acetaldehyde, } \\
\text { ammonium chloride, cyproconazole }\end{array}$ & $\begin{array}{l}20106945,17331233,22378314, \\
21871943,19150397,19484750, \\
22576693,21233418,21177773, \\
21296121,17361019,22634333, \\
20406850,22634333,16483693, \\
22045034\end{array}$ \\
\hline FAR2 & $\begin{array}{l}\text { rs } 1849374^{*} \\
\text { rs } 17439813 \\
\text { rs } 10506041\end{array}$ & $\begin{array}{l}\text { arsenite, benzo(a)pyrene, formaldehyde, } \\
\text { methylmercuric chloride, potassium chromate(VI), } \\
\text { testosterone, tetrachlorodibenzodioxin, aluminum }\end{array}$ & $\begin{array}{c}23974009,20064835,23649840, \\
23179753,22079256,21592394, \\
21998131,21298039\end{array}$ \\
\hline TMEM244 & rs6904762 & air pollutants & 21757418 \\
\hline
\end{tabular}




\begin{tabular}{|c|c|c|c|}
\hline Gene/region & SNP & Chemical interaction & PubMed IDs \\
\hline ACACA & $\begin{array}{l}\text { rs } 12948120 \\
\text { rs } 8081866 \\
\text { rs } 2542660 \\
\text { rs } 2542653\end{array}$ & $\begin{array}{l}\text { aflatoxin B1, dihydrotestosterone, arsenite, } \\
\text { benzo(a)pyrene, estradiol, formaldehyde, hydrogen } \\
\text { peroxide, niclosamide, tetrachlorodibenzodioxin, } \\
\text { 1,4-bis(2-(3,5-dichloropyridyloxy))benzene, 2,4- } \\
\text { dinitrotoluene, 2,6-dinitrotoluene, acetaminophen, } \\
\text { ammonium chloride, aristolochic acids, atrazine, } \\
\text { benzene, bisphenol A, bis(tri-n-butyltin)oxide, } \\
\text { chlorpyrifos, decamethrin, ethanol, ethinyl } \\
\text { estradiol, flusilazole, insecticides, } \\
\text { lipopolysaccharides, 2,6-dinitrotoluene, ammonium } \\
\text { chloride, ethanol, nicotine }\end{array}$ & $\begin{array}{l}20106945,18801408,23974009, \\
20106945,23019147,23649840, \\
21179406,22576131,21632981, \\
24878338,21346803,20406850, \\
17562736,16483693,21167265, \\
24440342,19483382,21932408, \\
22174045,21356183,23401056, \\
23692951,24440342,22382673, \\
23401056,22025971,20406850, \\
16483693,23692951,17635921\end{array}$ \\
\hline L3MBTL3 & rs6904762 & $\begin{array}{l}\text { potassium chromate(VI), acetaminophen, estradiol, } \\
\text { copper sulfate, tetrachlorodibenzodioxin }\end{array}$ & $\begin{array}{c}22079256,17562736,20068009, \\
19789285,21570461\end{array}$ \\
\hline ZNF706 & rs506389* & $\begin{array}{c}\text { atrazine, benzo(a)pyrene, copper sulfate, bisphenol } \\
\text { A }\end{array}$ & $\begin{array}{c}22378314,20064835,19549813, \\
24534842\end{array}$ \\
\hline SLC6A1 & $\begin{array}{l}\text { rs } 349714^{*} \\
\text { rs349707* }\end{array}$ & $\begin{array}{l}\text { aflatoxin B1, estradiol, agrochemicals, herbicides, } \\
\text { lipopolysaccharides, progesterone, toluene }\end{array}$ & $\begin{array}{c}20106945,20106945,22467014, \\
22467014,21219332,20631399, \\
21827849\end{array}$ \\
\hline HRH1 & $\begin{array}{l}\text { rs349714* } \\
\text { rs349707* }\end{array}$ & $\begin{array}{l}\text { PCB, aflatoxin B1, asbestos, benzo(a)pyrene, } \\
\text { benzo(a)pyrene diol epoxide, lipopolysaccharides, } \\
\text { particulate matter, vehicle emissions, endosulfan, } \\
\text { tetrachlorodibenzodioxin }\end{array}$ & $\begin{array}{c}11238657,22100608,22398240, \\
20064835,20382639,21255012, \\
10051702,10051702,16263506, \\
21570461\end{array}$ \\
\hline$V A P A$ & rs206457 & $\begin{array}{l}\text { benzene, acetaminophen, benzo(a)pyrene, bisphenol } \\
\text { A, dibutyl phthalate, particulate matter, } \\
\text { tetrachlorodibenzodioxin, vehicle emissions }\end{array}$ & $\begin{array}{l}19162166,17562736,21569818, \\
21786754,17379624,17295261, \\
21570461,17295261\end{array}$ \\
\hline$A P C D D 1$ & rs206457 & $\begin{array}{l}\text { benzo(a)pyrene diol epoxide, copper sulfate, } \\
\text { progesterone, tetrachlorodibenzodioxin, trichostatin } \\
\text { A, acetaminophen, estradiol, carbon tetrachloride, } \\
\text { toluene }\end{array}$ & $\begin{array}{l}20382639,19549813,20864642, \\
22298810,24935251,17562736, \\
14664709,17484886,22967744\end{array}$ \\
\hline
\end{tabular}




\begin{tabular}{|c|c|c|c|}
\hline Gene/region & SNP & Chemical interaction & PubMed IDs \\
\hline$P R L R$ & rs37389 & $\begin{array}{l}\text { arsenite, azaspiracid, benzo(a)pyrene, estradiol, } \\
\text { hydrogen peroxide, potassium chromate(VI), } \\
\text { progesterone, methoxychlor, 2,4-dinitrotoluene, } \\
\text { PCBs, acetaminophen, aflatoxin B1, benzene, } \\
\text { bisphenol A, cadmium, carbon tetrachloride, } \\
\text { clofibric acid, , cyproconazole, dibutyl phthalate, } \\
\text { diethylhexyl phthalate, diethylnitrosamine, } \\
\text { diethylstilbestrol, epoxiconazole, ethinyl estradiol, } \\
\text { fumonisin B1, lead acetate, 2-methoxyethanol, } \\
\text { methylmercury II, progesterone, propiconazole, } \\
\text { sodium arsenite, sulpiride, sumatriptan, } \\
\text { testosterone, tetrachlorodibenzodioxin, } \\
\text { trichloroethylene }\end{array}$ & $\begin{array}{l}23974009,20547569,22316170, \\
20660070,18951874,22079256, \\
20660070,19414516,21346803, \\
19467301,20959002,17562736, \\
19770486,17905399,22349186, \\
24067728,12734012,17602206, \\
19770486,22334560,21266533, \\
19850644,22129741,22349186, \\
12655037,21864555,23303685, \\
21061450,17905399,16251485, \\
22937105,18467677,16107292, \\
18162529,20844152,21570461, \\
17905399\end{array}$ \\
\hline FAM124A & rs9563035 & $\begin{array}{c}\text { atrazine, benzo(a)pyrene, copper sulfate, bisphenol } \\
\text { A, tetrachlorodibenzodioxin }\end{array}$ & $\begin{array}{c}22378314,22316170,19549813, \\
19770486\end{array}$ \\
\hline$L R P 2$ & rs 2544371 & $\begin{array}{l}\text { bisphenol A, ethinyl estradiol, hydrogen peroxide, } \\
\text { tetrachlorodibenzodioxin, PCB, cycloheximide, } \\
\text { particulate matter, progesterone, pseudocumene }\end{array}$ & $\begin{array}{c}20170705,20170705,20044591, \\
22298810,23994337,20576581, \\
19176365,17251523\end{array}$ \\
\hline BBS5 & rs2544371 & $\begin{array}{l}\text { copper sulfate, estrogens, acetaminophen, dibutyl } \\
\text { phthalate, dioxins, furan, progesterone, } \\
\text { tetrachlorodibenzodioxin }\end{array}$ & $\begin{array}{c}19549813,21233418,17562736, \\
21266533,20463971,22079235 \\
19690047,21570461\end{array}$ \\
\hline SOX5 & rs 7294845 & $\begin{array}{l}\text { benzo(a)pyrene, arsenite, benzo(a)pyrene diol } \\
\text { epoxide, bisphenol A, copper sulfate, vomitoxin, } \\
\text { acetaminophen, carbon tetrachloride, bis(2- } \\
\text { ethylhexyl) phthalate, ethinyl estradiol, } \\
\text { methylmercury, mustard gas, polychlorinated } \\
\text { biphenyls, potassium dichromate, propiconazole, } \\
\text { tetrachlorodibenzodioxin, tobacco smoke pollution }\end{array}$ & $\begin{array}{l}22316170,23974009,20382639, \\
20170705,19549813,23792671, \\
17562736,17484886,19850644, \\
17555576,20020106,15674844, \\
20020106,23608068,21278054, \\
21570461,20133372\end{array}$ \\
\hline$R Y B P$ & rs11708505 & $\begin{array}{c}\text { acetaminophen, copper sulfate, formaldehyde, } \\
\text { hydrogen peroxide, benzo(a)pyrene, dibutyl } \\
\text { phthalate }\end{array}$ & $\begin{array}{l}21420995,19549813,23649840, \\
20044591,20504355,21266533\end{array}$ \\
\hline
\end{tabular}




\begin{tabular}{|c|c|c|c|}
\hline Gene/region & SNP & Chemical interaction & PubMed IDs \\
\hline SHQ1 & rs11708505 & atrazine, bisphenol A, tetrachlorodibenzodioxin & $22378314,21786754,21570461$ \\
\hline HHAT & rs6540610 & $\begin{array}{c}\text { aflatoxin B1, benzo(a)pyrene, copper sulfate, } \\
\text { formaldehyde, hydrogen peroxide, } \\
\text { tetrachlorodibenzodioxin }\end{array}$ & $\begin{array}{l}20106945,20106945,21632981, \\
20106945,23649840,23649840, \\
20106945,21632981\end{array}$ \\
\hline GRIA2 & $\begin{array}{l}\text { rs } 1550017 \\
\text { rs } 12054636\end{array}$ & $\begin{array}{l}\text { arsenite, aflatoxin B1, estradiol, bisphenol A, } \\
\text { chlorpyrifos, dieldrin, ethanol, lead, progesterone, } \\
\text { tetrachlorodibenzodioxin, toluene }\end{array}$ & $\begin{array}{l}23974009,23630614,22154832, \\
22504913,11772933,23719929, \\
22154832,16922920,21827849\end{array}$ \\
\hline GRIK1 & rs468771 & $\begin{array}{l}\text { benzo(a)pyrene, tetrachlorodibenzodioxin, } \\
\text { ammonium chloride, progesterone }\end{array}$ & $\begin{array}{c}21802500,21802500,16483693, \\
19690047\end{array}$ \\
\hline SUB1 & rs864674 & $\begin{array}{c}\text { arsenic trioxide, atrazine, formaldehyde, mercury, } \\
\text { tetrachlorodibenzodioxin, benzo(a)pyrene, } \\
\text { bisphenol A, dioxins, ethinyl estradiol, fipronil, } \\
\text { nicotine }\end{array}$ & $\begin{array}{c}20458559,22378314,23649840, \\
19937285,22574217,22228805, \\
21786754,20463971,17555576, \\
23962444,17456735\end{array}$ \\
\hline NPR3 & rs864674 & $\begin{array}{l}\text { aflatoxin B1, benzo(a)pyrene diol, estradiol, copper } \\
\text { sulfate, fonofos, parathion, progesterone, smoke, } \\
\text { terbufos, PCB, acetaminophen, arsenic, } \\
\text { benzo(a)pyrene, bisphenol A, carbon tetrachloride, } \\
\text { lipopolysaccharides, nitrofen, ozone, } \\
\text { tetrachlorodibenzodioxin, tobacco smoke pollution }\end{array}$ & $\begin{array}{c}22100608,20382639,23019147, \\
19549813,22847954,20864642, \\
21095227,22847954,24935251, \\
17562736,19654921,15034205, \\
19228888,17484886,22128259, \\
10898224,19555225,15034205, \\
20133372\end{array}$ \\
\hline KMT2B & rs8106959 & $\begin{array}{l}\text { asbestos, benzene, mercury, } \\
\text { tetrachlorodibenzodioxin, uranium compounds, } \\
\text { aflatoxin B1, methylcholanthrene }\end{array}$ & $\begin{array}{c}17331233,19162166,16823088, \\
21296121,19654044,19770486, \\
18804290\end{array}$ \\
\hline GPC6 & rs9561489 & $\begin{array}{l}\text { acetaminophen, aflatoxin B1, arsenite, } \\
\text { benzo(a)pyrene, benzo(a)pyrene diol, testosterone, } \\
\text { carbon tetrachloride }\end{array}$ & $\begin{array}{c}\text { 21420995, 20106945, 23974009, } \\
\text { 20106945, 21632981, 20382639, } \\
\text { 20106945, 23300844, 23179753, } \\
\text { 24383497, 24935251, 17484886 }\end{array}$ \\
\hline MYO18A & rs9897342 & $\begin{array}{l}\text { estradiol, bisphenol A, copper sulfate, } \\
\text { acetaminophen, aflatoxin B1, benzo(a)pyrene, } \\
\text { carbon tetrachloride, tetrachlorodibenzodioxin }\end{array}$ & $\begin{array}{c}23019147,19549813,17562736, \\
19770486,22228805,17484886, \\
21570461\end{array}$ \\
\hline
\end{tabular}




\begin{tabular}{|c|c|c|c|}
\hline Gene/region & SNP & Chemical interaction & PubMed IDs \\
\hline$A L X 3$ & rs 756134 & fonofos, parathion, terbufos & 22847954, 22847954, 22847954 \\
\hline$A B C B 11$ & $\begin{array}{l}\text { rs552976 } \\
\text { rs563694 }\end{array}$ & $\begin{array}{l}\text { acetaminophen, aflatoxin B1, diethylstilbestrol, } \\
\text { ethinyl estradiol, lipopolysaccharides, progesterone, } \\
\text { tetrachlorodibenzodioxin, benzo(a)pyrene, carbon } \\
\text { tetrachloride, potassium dichromate }\end{array}$ & $\begin{array}{l}21420995,22100608,20829430, \\
15205115,24014644,16837569, \\
19770486,17522070,23608068\end{array}$ \\
\hline TNFRSF19 & rs9510793 & $\begin{array}{l}\text { arsenic, benzo(a)pyrene, benzo(a)pyrene diol } \\
\text { epoxide, estradiol, potassium chromate(VI), } \\
\text { progesterone, tetrachlorodibenzodioxin, PCB, } \\
\text { acetaminophen, cyproconazole, epoxiconazole, } \\
\text { methoxychlor, methylmercuric chloride, DDT, } \\
\text { propiconazole }\end{array}$ & $\begin{array}{c}24675094,20106945,21632981, \\
20382639,23649840,22079256, \\
20660070,21632981,24383497, \\
21851831,17562736,22334560, \\
23970803,20829426,20864626, \\
18925944,21278054,22334560, \\
23970803,\end{array}$ \\
\hline N4BP2 & rs61748746 & $\begin{array}{c}\text { acetaminophen, aflatoxin B1, benzo(a)pyrene, } \\
\text { formaldehyde, PCB, bisphenol A, } \\
\text { tetrachlorodibenzodioxin }\end{array}$ & $\begin{array}{l}21420995,20106945,20106945, \\
23649840,21786754,21570461\end{array}$ \\
\hline G6PC2 & $\begin{array}{l}\mathrm{rs563694} \\
\text { rs560887 }\end{array}$ & cyclophosphamide & 15331540 \\
\hline SMARCA2 & rs7025331 & $\begin{array}{l}\text { acetaminophen, arsenic trioxide, benzo(a)pyrene, } \\
\text { copper sulfate, potassium chromate(VI), PCB, } \\
\text { bisphenol A, dibutyl phthalate, ethinyl estradiol, } \\
\text { pentachlorophenol, tetrachlorodibenzodioxin }\end{array}$ & $\begin{array}{c}21420995,20458559,20106945, \\
19549813,22079256,19114083, \\
23359474,23359474,17942748, \\
24642059,17942748\end{array}$ \\
\hline DOCK1 & rs7917910 & $\begin{array}{l}\text { acetaminophen, aflatoxin B1, atrazine, } \\
\text { benzo(a)pyrene, copper sulfate, dibutyl phthalate, } \\
\text { lead acetate, methylmercuric chloride, progesterone, } \\
\text { sodium arsenite, tetrachlorodibenzodioxin }\end{array}$ & $\begin{array}{c}17562736,21641981,22378314, \\
22316170,19549813,21266533, \\
20542052,23103053,19690047, \\
21297353,21570461\end{array}$ \\
\hline PDIA5 & rs9873177 & $\begin{array}{c}\text { acetaminophen, atrazine, diazinon, benzo(a)pyrene, } \\
\text { bisphenol A, clofibric acid, diethylhexyl phthalate, } \\
\text { pentachlorophenol, propiconazole, } \\
\text { tetrachlorodibenzodioxin }\end{array}$ & $\begin{array}{c}21420995,22378314,22964155, \\
23179753,24383497,19770486, \\
21786754,21318169,21278054, \\
18495758\end{array}$ \\
\hline
\end{tabular}




\begin{tabular}{|c|c|c|c|}
\hline Gene/region & SNP & Chemical interaction & PubMed IDs \\
\hline SPATA20 & rs739924 & $\begin{array}{l}\text { arsenic trioxide, benzo(a)pyrene, benzo(a)pyrene } \\
\text { diol epoxide, cadmium, copper sulfate, } \\
\text { tetrachlorodibenzodioxin, bisphenol A, } \\
\text { chlorpyrifos, progesterone }\end{array}$ & $\begin{array}{l}20707922,21632981,19150397, \\
24376830,19549813,20106945, \\
21632981,24586524,21356183, \\
23012394\end{array}$ \\
\hline$M G M T$ & rs1978759 & $\begin{array}{l}\text { aflatoxin } \mathrm{B} 1 \text {, benzo(a)pyrene, ethylene dibromide, } \\
\text { dust, fonofos, parathion, polycyclic aromatic } \\
\text { hydrocarbon, acetaminophen, ammonium chloride, } \\
\text { arsenite, estradiol, bisphenol A, carbon } \\
\text { tetrachloride, diethylstilbestrol, furan, } \\
\text { methylnitrosourea, ozone, praziquantel, sodium } \\
\text { arsenite, tetrachlorodibenzodioxin }\end{array}$ & $\begin{array}{l}20106945,20064835,12151404, \\
17805423,8823159,23543013, \\
12883083,16483693,18929588, \\
20965277,20965277,17484886, \\
15890375,22507866,20965277, \\
23358140,18636392,15890375, \\
21813463,11241755,21215274\end{array}$ \\
\hline CSRNP3 & rs 729867 & PCB, dibutyl phthalate & 19114083,21266533 \\
\hline CSMD1 & rs4875742 & aflatoxin B1, tetrachlorodibenzodioxin & 23630614,21570461 \\
\hline$S C N 3 B$ & rs 1720326 & $\begin{array}{l}\text { aflatoxin B1, methylcholanthrene, PCB, arsenic, } \\
\text { benzo(a)pyrene, estradiol, bisphenol A, carbon } \\
\text { tetrachloride, cyfluthrin, cypermethrin, dibutyl } \\
\text { phthalate, fenvalerate, nitrofen, Ozone }\end{array}$ & $\begin{array}{l}20106945,20348232,19114083, \\
19654921,21569818,23557687, \\
23359474,12629582,18538810, \\
18538810,23359474,18538810, \\
20638522,17095637\end{array}$ \\
\hline ZNF202 & rs 1720326 & estradiol, formaldehyde & 20106945,20655997 \\
\hline SORCS2 & rs3857193 & $\begin{array}{l}\text { aflatoxin B1, benzo(a)pyrene, formaldehyde, } \\
\text { hydrogen peroxide, tetrachlorodibenzodioxin, } \\
\text { tobacco smoke pollution }\end{array}$ & $\begin{array}{c}22100608,21871943,23649840, \\
20044591, \quad 24383497,19933214, \\
20133372\end{array}$ \\
\hline CCDC25 & rs6987111 & $\begin{array}{c}\text { copper sulfate, acetaminophen, benzo(a)pyrene, } \\
\text { bisphenol A, dibutyl phthalate, testosterone, } \\
\text { tetrachlorodibenzodioxin }\end{array}$ & $\begin{array}{l}19549813,17562736,22228805, \\
21786754,21266533,20844152, \\
21570461\end{array}$ \\
\hline ZNF611 & rs4801931 & formaldehyde, smoke & 23649840,21095227 \\
\hline ZNF652 & $\begin{array}{l}\text { rs } 12940887 \\
\text { rs } 7225787 \\
\text { rs } 4794031 \\
\text { rs } 11079867\end{array}$ & $\begin{array}{c}\text { acetaminophen, aflatoxin B1, benzo(a)pyrene, } \\
\text { benzo(a)pyrene diol epoxide, estradiol, copper } \\
\text { sulfate, formaldehyde, hydrogen peroxide, } \\
\text { progesterone, tetrachlorodibenzodioxin, dibutyl } \\
\text { phthalate }\end{array}$ & $\begin{array}{c}21420995,22100608,22316170, \\
20018196,17404688,19549813, \\
20655997,23649840,23649840, \\
17404688,20106945,21632981, \\
23179753,21266533\end{array}$ \\
\hline
\end{tabular}




\begin{tabular}{|c|c|c|c|}
\hline Gene/region & SNP & Chemical interaction & PubMed IDs \\
\hline SMC4 & rs4680582 & $\begin{array}{l}\text { acetaminophen, aflatoxin B1, arsenic trioxide, } \\
\text { benzene, benzo(a)pyrene diol epoxide, estradiol, } \\
\text { bisphenol A, hydrogen peroxide, mercury, } \\
\text { polychlorinated biphenyls, progesterone, } \\
\text { testosterone, tetrachlorodibenzodioxin }\end{array}$ & $\begin{array}{l}2230336,20106945,17547211, \\
\text { 19162166, 19150397, 20018196, } \\
\text { 20382639, 16474171, 19167446, } \\
\text { 23410634, 17547211, 23649840, } \\
16474171,21795739,18691609\end{array}$ \\
\hline HIP1 & rs6962352 & $\begin{array}{l}\text { acetaminophen, benzo(a)pyrene, copper sulfate, } \\
\text { potassium chromate(VI), tetrachlorodibenzodioxin, } \\
\text { dibutyl phthalate, ethinyl estradiol, }\end{array}$ & $\begin{array}{l}21420995,20106945,19549813, \\
22079256,21934132,21266533, \\
20227414\end{array}$ \\
\hline SYNRG & rs4074605 & $\begin{array}{l}\text { formaldehyde, acetaminophen, chlorpyrifos, } \\
\text { tetrachlorodibenzodioxin }\end{array}$ & $\begin{array}{c}20655997,17562736,18668222, \\
21570461\end{array}$ \\
\hline TXNRD1 & rs10778311 & $\begin{array}{l}\text { acetaminophen, arsenic trioxide, arsenite, asbestos, } \\
\text { benzo(a)pyrene, benzo(a)pyrene diol epoxide, } \\
\text { estradiol, caffeine, chlorobenzene, chloropicrin, } \\
\text { copper sulfate, cupric chloride, cycloheximide, } \\
\text { cylindrospermopsin, diquat, epichlorohydrin, } \\
\text { glycidamide, hydrogen peroxide, hydroquinone, } \\
\text { mercuric chloride, mercury, methylmercuric } \\
\text { chloride, tetrachlorodibenzodioxin, tobacco smoke } \\
\text { pollution }\end{array}$ & $\begin{array}{l}21420995,17640917,18550526, \\
18687144,20106945,21632981, \\
22316170,19150397,23665804, \\
18444173,19800902,19549813, \\
21172416,24211530,17822822, \\
18951874,15452088,24816296, \\
17547211,24816296,22298810\end{array}$ \\
\hline ZBTB16 & rs 10128580 & $\begin{array}{l}\text { acetaminophen, estradiol, mercury, progesterone, } \\
\text { arsenic, chlorpyrifos, cyproconazole, flusilazole, } \\
\text { hexaconazole, mercuric chloride, ozone, } \\
\text { progesterone, propiconazole, sodium arsenite, } \\
\text { tetrachlorodibenzodioxin, tobacco smoke pollution }\end{array}$ & $\begin{array}{c}22230336,17404688,19937285, \\
17404688,19654921,20350560, \\
22045034,20353558,17460151, \\
17251523,21278054,17682005, \\
20055451,20133372\end{array}$ \\
\hline DLGAP1 & rs 7243843 & $\begin{array}{l}\text { aflatoxin B1, formaldehyde, } \\
\text { tetrachlorodibenzodioxin, bisphenol A, dibutyl } \\
\text { phthalate, environmental pollutants, progesterone }\end{array}$ & $\begin{array}{l}22100608,23649840,21296121, \\
24820113,21266533,20064776, \\
22238285\end{array}$ \\
\hline EYA4 & rs9373051 & $\begin{array}{l}\text { benzo(a)pyrene diol epoxide, estradiol, bisphenol } \\
\text { A, lead acetate, tetrachlorodibenzodioxin, tobacco } \\
\text { smoke pollution }\end{array}$ & $\begin{array}{c}19150397,23179753,24383497, \\
23557687,21786754,22641619, \\
19933214,20133372\end{array}$ \\
\hline
\end{tabular}




\begin{tabular}{|c|c|c|c|}
\hline Gene/region & SNP & Chemical interaction & PubMed IDs \\
\hline ADAMTS12 & rs 7700370 & $\begin{array}{c}\text { acetaminophen, smoke, benzo(a)pyrene, carbon } \\
\text { tetrachloride }\end{array}$ & $\begin{array}{c}22230336,21095227,24935251, \\
20713471,17484886\end{array}$ \\
\hline DOK5 & rs910850 & $\begin{array}{l}\text { arsenic trioxide, arsenite, } \mathrm{PCB} \text {, benzo(a)pyrene, } \\
\text { dibutyl phthalate, potassium chromate(VI) }\end{array}$ & $\begin{array}{l}20458559,23974009,24383497, \\
24935251,23829299,14625279, \\
21266533,14625279\end{array}$ \\
\hline SLC38A1 & rs 12822012 & $\begin{array}{l}\text { acetaminophen, atrazine, benzo(a)pyrene, } \\
\text { benzo(a)pyrene diol epoxide, copper sulfate, DDT, } \\
\text { chlorpyrifos, heptachlor, lipopolysaccharides, } \\
\text { progesterone, tetrachlorodibenzodioxin }\end{array}$ & $\begin{array}{c}21420995,22378314,20064835, \\
20382639,19549813,18204070, \\
18668222,23153324,21645290, \\
20726854,19770486,\end{array}$ \\
\hline $\mathrm{CDH} 2$ & rs 7237664 & $\begin{array}{l}\text { arsenite, benzo(a)pyrene, benzo(a)pyrene diol } \\
\text { epoxide, DDT, estrogens, hydrogen peroxide, } \\
\text { acetaminophen, aflatoxin B1, agrochemicals, } \\
\text { bisphenol A, dibutyl phthalate, herbicides, mercuric } \\
\text { chloride, methylcholanthrene, pentachlorophenol, } \\
\text { progesterone, tetrachlorodibenzodioxin, tobacco } \\
\text { smoke pollution }\end{array}$ & $\begin{array}{c}23974009,17690111,19150397, \\
24820114,15473135,12419474, \\
17562736,19770486,22467014, \\
21786754,21266533,22467014, \\
21126564,21163286,17556502, \\
24680724,20133372\end{array}$ \\
\hline GOLGA8B & rs7163190 & DDT, hydrogen peroxide & 25014179,21179406 \\
\hline GJD2 & rs7163190 & tetrachlorodibenzodioxin & 20055451 \\
\hline HSD17B2 & rs11648233 & $\begin{array}{c}\text { acetaminophen, aflatoxin B1, arsenic trioxide, } \\
\text { benzo(a)pyrene, benzo(a)pyrene diol epoxide, } \\
\text { estradiol, copper sulfate, hydrogen peroxide, } \\
\text { progesterone, testosterone, } \\
\text { tetrachlorodibenzodioxin, atrazine, bisphenol A, } \\
\text { carbon tetrachloride, hexachlorobenzene, mercuric } \\
\text { chloride, methoxychlor }\end{array}$ & $\begin{array}{l}21420995,20106945,17547211, \\
20106945,20382639,21972996, \\
19549813,20044591,20660070, \\
16054388,22298810,16249480, \\
22285353,22285353,16239168, \\
15159207,16507785,22285353\end{array}$ \\
\hline
\end{tabular}

$\mathrm{SNP}=$ single nucleotide polymorphism 


\section{Appendix Table 11: Genes showing gender-specific associations (ranked by their OR) and their interactions with chemicals in studies curated in the Comparative Toxicological Database}

\begin{tabular}{|c|c|c|c|}
\hline Gene/region & SNP & Chemical interaction & PubMed IDs \\
\hline$H L A-D Q A 1$ & $\begin{array}{l}\text { rs12722042 } \\
\text { rs12722039 }\end{array}$ & $\begin{array}{l}\text { PCB, arsenic, benzene, lipopolysaccharides, } \\
\text { mercury, progesterone, aluminum }\end{array}$ & $\begin{array}{l}20638727,18414638,16188091, \\
22235868,19690047,21298039\end{array}$ \\
\hline ADAM28 & $\begin{array}{l}\text { rs } 11992342 \\
\text { rs } 7829965 \\
\text { rs36041430 }\end{array}$ & $\begin{array}{l}\text { benzo(a)pyrene, estradiol, progesterone, } \\
\text { tetrachlorodibenzodioxin, bisphenol A }\end{array}$ & $\begin{array}{l}22316170,20660070,20660070, \\
22298810,24935251,24586524\end{array}$ \\
\hline UMODL1 & rs80040922 & tetrachlorodibenzodioxin, vehicle emissions & 21570461,23940539 \\
\hline CECR5 & rs35665085 & acetaminophen, 2,4-dinitrotoluene & 21420995,21346803 \\
\hline CNTN3 & rs 143021649 & $\begin{array}{l}\text { acetaminophen, aflatoxin } \mathrm{B} 1 \text {, benzo(a)pyrene diol } \\
\text { epoxide, progesterone, trichostatin } \mathrm{A}, \mathrm{PCB}, \\
\text { ammonium chloride, ethinyl estradiol }\end{array}$ & $\begin{array}{l}21420995,22100608,20382639, \\
21795739,24935251,23179753, \\
19114083,16483693,17557909\end{array}$ \\
\hline PROS1 & rs6795524 & $\begin{array}{l}\text { acetaminophen, benzo(a)pyrene diol, estradiol, } \\
\text { copper sulfate, particulate matter, PCBs, } \\
\text { tetrachlorodibenzodioxin, vehicle emissions, } \\
\text { asbestos, benzo(a)pyrene, bisphenol A, carbon } \\
\text { tetrachloride, diuron, fipronil, 2,4-dinitrotoluene, } \\
\text { 2,6-dinitrotoluene, ammonium chloride, ethinyl } \\
\text { estradiol, silicon dioxide }\end{array}$ & $\begin{array}{c}22230336,20382639,19619570, \\
19549813,17987463,21334430, \\
20436886,19619570,21934132, \\
24935251,23179753,17987463, \\
22352330,22228805,21786754, \\
17484886,23962444,21346803, \\
21346803,16483693,15288212, \\
19073995\end{array}$ \\
\hline FAT4 & rs 10003468 & $\begin{array}{l}\text { potassium chromate(VI), benzo(a)pyrene, dibutyl } \\
\text { phthalate, tetrachlorodibenzodioxin, toluene }\end{array}$ & $\begin{array}{c}22079256,21569818,21266533, \\
19933214,22967744\end{array}$ \\
\hline CNRIP1 & rs3732042 & $\begin{array}{l}\text { PCB, Acetaminophen, Aflatoxin B1, trichostatin A, } \\
\text { Valproic Acid, Tetrachlorodibenzodioxin, Tretinoin }\end{array}$ & $\begin{array}{c}23146750,21420995,22100608, \\
24935251,23179753,19654925, \\
20488242\end{array}$ \\
\hline GRK4 & rs34022679 & $\begin{array}{l}\text { arsenic trioxide, } \mathrm{PCBs} \text {, benzo(a)pyrene, ethinyl } \\
\text { estradiol, tetrachlorodibenzodioxin }\end{array}$ & $\begin{array}{c}15761015,19114083,19654925, \\
17942748,17942748\end{array}$ \\
\hline PPA1 & rs 7097067 & $\begin{array}{c}\text { acetaminophen, copper sulfate, dust, } \\
\text { benzo(a)pyrene, estradiol, bisphenol A, furan, } \\
\text { pentachlorophenol, progesterone, } \\
\text { tetrachlorodibenzodioxin }\end{array}$ & $\begin{array}{l}22230336,19549813,17805423, \\
22228805,19484750,21786754, \\
22240984,23018184,21570461\end{array}$ \\
\hline
\end{tabular}


Appendix Table 11 continued....

\begin{tabular}{|c|c|c|c|}
\hline Gene/region & SNP & Chemical interaction & PubMed IDs \\
\hline TMEM106B & $\begin{array}{l}\mathrm{rs} 35912436^{*} \\
\mathrm{rs} 2160271^{*}\end{array}$ & $\begin{array}{c}\text { benzo(a)pyrene diol epoxide, hydrogen peroxide, } \\
\text { testosterone, PCB, acetaminophen, aflatoxin B1, } \\
\text { carbon tetrachloride, copper sulfate, } \\
\text { tetrachlorodibenzodioxin }\end{array}$ & $\begin{array}{c}20018196,18951874,21592394, \\
22298810,19467301,17562736, \\
19770486,17484886,18579281, \\
22298810\end{array}$ \\
\hline VWDE & $\begin{array}{l}\text { rs35912436* } \\
\text { rs } 2160271^{*}\end{array}$ & benzo(a)pyrene, tetrachlorodibenzodioxin & 22316170,21570461 \\
\hline KATNAL1 & rs 17588454 & $\begin{array}{c}\text { acetaminophen, copper sulfate, potassium } \\
\text { chromate(VI), bisphenol A, fipronil, } \\
\text { tetrachlorodibenzodioxin }\end{array}$ & $\begin{array}{c}22230336,19549813,22079256, \\
21786754,21570461\end{array}$ \\
\hline PLEKHG1 & $\begin{array}{l}\text { rs } 17080410 \\
\text { rs61742396 }\end{array}$ & $\begin{array}{l}\text { arsenic trioxide, formaldehyde, methylmercuric } \\
\text { chloride, nicotine, acetaminophen }\end{array}$ & $\begin{array}{c}20458559,23649840,23179753, \\
18438686,17562736\end{array}$ \\
\hline EXO5 & rs35672330 & $\begin{array}{c}\text { acetaminophen, atrazine, copper sulfate, } \\
\text { tetrachlorodibenzodioxin }\end{array}$ & $\begin{array}{l}22230336,22378314,19549813, \\
22574217,\end{array}$ \\
\hline$T L L 1$ & rs1503292 & $\begin{array}{c}\text { lipopolysaccharides, tetrachlorodibenzodioxin, } \\
\text { thiram }\end{array}$ & $12057914,19933214,20530235$ \\
\hline KCNH7 & rs 1978526 & $\begin{array}{l}\text { uranium compounds, } \mathrm{PCB} \text {, aflatoxin } \mathrm{B} 1 \text {, carbon } \\
\text { tetrachloride, paraquat, perfluorooctane sulfonic } \\
\text { acid }\end{array}$ & $\begin{array}{l}19654044,19114083,23630614, \\
12734012,22563483,19162173\end{array}$ \\
\hline CD180 & rs6887604 & $\begin{array}{l}\text { acetaminophen, benzo(a)pyrene, estradiol, } \\
\text { progesterone, testosterone, biological toxins }\end{array}$ & $\begin{array}{l}17562736,22228805,19693291, \\
19693291,19693291,19682533\end{array}$ \\
\hline DRD1 & rs 10063286 & $\begin{array}{l}\text { fonofos, parathion, terbufos, } \\
\text { tetrachlorodibenzodioxin, formaldehyde, } \\
\text { methoxychlor }\end{array}$ & $\begin{array}{c}22847954,22847954,19901195, \\
17434604\end{array}$ \\
\hline SFXN1 & rs10063286 & $\begin{array}{l}\text { acetaminophen, arsenite, benzo(a)pyrene diol } \\
\text { epoxide, bisphenol A, copper sulfate, formaldehyde, } \\
\text { PCB, estradiol, tetrachlorodibenzodioxin }\end{array}$ & $\begin{array}{l}21420995,23974009,19150397, \\
22576693,19549813,23649840, \\
20959002,16684588,21570461\end{array}$ \\
\hline NT5C3 & rs 16879126 & $\begin{array}{c}\text { acetaminophen, ethinyl estradiol, fipronil, lead, } \\
\text { tetrachlorodibenzodioxin }\end{array}$ & $\begin{array}{c}17562736,17942748,23962444, \\
16672222,21570461\end{array}$ \\
\hline
\end{tabular}


Appendix Table 11 continued....

\begin{tabular}{|c|c|c|c|}
\hline Gene/region & SNP & Chemical interaction & PubMed IDs \\
\hline ANK1 & $\begin{array}{l}\text { rs34265667 } \\
\text { rs34664882 }\end{array}$ & $\begin{array}{l}\text { mercury, testosterone, benzo(a)pyrene, estradiol, } \\
\text { dieldrin, tetrachlorodibenzodioxin }\end{array}$ & $\begin{array}{c}19937285,21592394,22228805 \\
20068009,17942748\end{array}$ \\
\hline GABARAPL1 & rs4764332 & $\begin{array}{l}\text { aflatoxin B1, air pollutants, atrazine, } \\
\text { benzo(a)pyrene, copper sulfate, hydrogen peroxide, } \\
\text { tetrachlorodibenzodioxin, tobacco smoke pollution, } \\
\text { acetaminophen, benzene, dibutyl phthalate, } \\
\text { progesterone }\end{array}$ & $\begin{array}{c}21641981,21757418,22378314, \\
22316170,19549813,20044591, \\
21632981,19101580,17562736, \\
15120971,21266533\end{array}$ \\
\hline KLRD1 & rs4764332 & $\begin{array}{c}\text { acetaminophen, arsenite, benzo(a)pyrene, } \\
\text { methotrexate, estradiol, tetrachlorodibenzodioxin, } \\
\text { tobacco smoke pollution }\end{array}$ & $\begin{array}{c}17093179,23974009,22316170, \\
17400583,19484750,21041162, \\
20133372\end{array}$ \\
\hline KCNH8 & rs7626907 & arsenite, lead & 23974009,19921347 \\
\hline EFHB & rs7626907 & atrazine, copper sulfate, estradiol, chlorpyrifos & $\begin{array}{c}22378314,19549813,19484750, \\
21356183\end{array}$ \\
\hline ACAA1 & rs 2229528 & $\begin{array}{c}\text { acetaminophen, aflatoxin B1, copper sulfate, } \\
\text { hydrogen peroxide, bisphenol A, cadmium, dibutyl } \\
\text { phthalate, furan, phthalic acids, } \\
\text { tetrachlorodibenzodioxin }\end{array}$ & $\begin{array}{c}22230336,20106945,19549813, \\
18951874,19287073,17327699, \\
21266533,15120968,15728792, \\
16960034\end{array}$ \\
\hline SCHIP1 & rs9842465 & $\begin{array}{c}\text { acetaminophen, atrazine, benzo(a)pyrene, } \\
\text { benzo(a)pyrene diol, copper sulfate, estrogens, } \\
\text { aflatoxin B1, carbon tetrachloride, dibutyl } \\
\text { phthalate, tetrachlorodibenzodioxin }\end{array}$ & $\begin{array}{c}21420995,22378314,20106945, \\
19150397,19549813,21233418, \\
23724009,19770486,17484886, \\
21266533,21570461\end{array}$ \\
\hline FGF12 & rs6796558 & $\begin{array}{l}\text { aflatoxin B1, arsenic, arsenic trioxide, arsenite, } \\
\text { fonofos, methotrexate, parathion, potassium } \\
\text { chromate(VI), terbufos, uranium compounds, PCB, } \\
\text { benzo(a)pyrene, carbon tetrachloride }\end{array}$ & $\begin{array}{l}22100608,24675094,22521957, \\
23974009,22847954,17400583, \\
22847954,22079256,22847954, \\
17045167,19654044,23179753, \\
19114083,22228805,17484886\end{array}$ \\
\hline MAST4 & rs55882716 & $\begin{array}{l}\text { acetaminophen, aflatoxin B1, cadmium chloride, } \\
\text { copper sulfate, dibutyl phthalate, formaldehyde, } \\
\text { smoke, testosterone, tetrachlorodibenzodioxin }\end{array}$ & $\begin{array}{l}17562736,21641981,12160620, \\
19549813,21266533,20655997, \\
21095227,21592394,22298810\end{array}$ \\
\hline
\end{tabular}


Appendix Table 11 continued....

\begin{tabular}{|c|c|c|c|}
\hline Gene/region & SNP & Chemical interaction & PubMed IDs \\
\hline CYFIP2 & rs 1030270 & $\begin{array}{l}\text { acetaminophen, aflatoxin B1, air pollutants, arsenic } \\
\text { trioxide, atrazine, benzo(a)pyrene, benzo(a)pyrene } \\
\text { diol, estradiol, cupric chloride, formaldehyde, } \\
\text { progesterone, bisphenol A, methylmercury } \\
\text { compounds, tetrachlorodibenzodioxin }\end{array}$ & $\begin{array}{c}22230336,20106945,21757418, \\
15894607,22378314,22316170, \\
20382639,20660070,17211630, \\
23649840,23649840,23649840, \\
20660070,15894607,17183730, \\
21786754,21664453,19759094, \\
15901671 \\
\end{array}$ \\
\hline SLC35F1 & rs205944 & dibutyl phthalate, tetrachlorodibenzodioxin & 21266533,21570461 \\
\hline IL20RA & rs381783 & $\begin{array}{l}\text { estradiol, progesterone, tetrachlorodibenzodioxin, } \\
\text { acetaminophen, benzo(a)pyrene, bisphenol A, } \\
\text { mercuric chloride, tetrachlorodibenzodioxin }\end{array}$ & $\begin{array}{c}20660070,23152189,23724009, \\
17562736,22228805,21786754, \\
18496552,21570461\end{array}$ \\
\hline KDELR2 & rs11980237 & $\begin{array}{l}\text { asbestos, atrazine, copper sulfate, formaldehyde, } \\
\text { acetaminophen, bisphenol A, cadmium sulfate, } \\
\text { ethinyl estradiol, propiconazole, sodium arsenate, } \\
\text { tetrachlorodibenzodioxin }\end{array}$ & $\begin{array}{c}18687144,22378314,19549813, \\
23649840,21786754,16221973, \\
17942748,21278054,16882884, \\
18495758\end{array}$ \\
\hline$D G K B$ & rs 720872 & progesterone, occupational air pollutants, & 21795739,19936710 \\
\hline AGMO & rs720872 & $\begin{array}{l}\text { aflatoxin B1, benzo(a)pyrene, copper sulfate, } \\
\text { acetaminophen, agrochemicals, diuron, herbicides, } \\
\text { mercuric Chloride, tetrachlorodibenzodioxin }\end{array}$ & $\begin{array}{l}22100608,21871943,19549813, \\
17562736,22467014,21551480, \\
22467014,20353558,19770486\end{array}$ \\
\hline RGS3 & rs147596194 & $\begin{array}{c}\text { acetaminophen, asbestos, benzo(a)pyrene, estradiol, } \\
\text { diazinon, hydrogen peroxide, progesterone, } \\
\text { arsenite, benzene, carbon tetrachloride, dibutyl } \\
\text { phthalate, methylmercury II, } \\
\text { tetrachlorodibenzodioxin }\end{array}$ & $\begin{array}{l}21420995,17331233,21714911, \\
20660070,23410634,21592394, \\
24935251,18929588,17905399, \\
17484886,21266533,17905399, \\
21570461\end{array}$ \\
\hline MAMDC4 & rs61745456 & benzo(a)pyrene, tetrachlorodibenzodioxin & 22228805,22298810 \\
\hline WDR87 & $\begin{array}{l}\text { rs117923468 } \\
\text { rs182319278 }\end{array}$ & tobacco smoke pollution & 18087596 \\
\hline
\end{tabular}


Appendix Table 11 continued....

\begin{tabular}{|c|c|c|c|}
\hline Gene/region & SNP & Chemical interaction & PubMed IDs \\
\hline$A P P$ & rs985456 & $\begin{array}{l}\text { benzene, benzo(a)pyrene, cadmium, estradiol, } \\
\text { fonofos, hydrogen peroxide, ibuprofen, iron, lead, } \\
\text { mercury, methotrexate, nicotine, ozone, parathion, } \\
\text { particulate matter, terbufos, uranium, aluminum } \\
\text { chloride, cadmium chloride, carbon tetrachloride, } \\
\text { dichlorvos, diuron, fipronil, lead acetate, } \\
\text { lipopolysaccharides, nickel, nicotine, DDT, } \\
\text { progesterone, propiconazole, sodium arsenite, } \\
\text { tetrachlorodibenzodioxin, toluene }\end{array}$ & $\begin{array}{l}15707952,22316170,16266835, \\
12963085,22847954,21179406, \\
12777371,16308480,22313790, \\
10617124,12182891,17400583, \\
16627626,15513908,22847954, \\
15513908,18482095,19840844, \\
17484886,21551480,23962444, \\
17277887,11080519,16230335, \\
22764079,15919760,21952557, \\
17135361,17984292,19162173, \\
22484513,17988898,21440049\end{array}$ \\
\hline CYYR1 & rs985456 & $\begin{array}{c}\text { arsenite, methylmercuric chloride, silicon dioxide, } \\
\text { dibutyl phthalate, fipronil, } \\
\text { tetrachlorodibenzodioxin, toluene }\end{array}$ & $\begin{array}{l}23974009,23179753,23806026, \\
21266533,23962444,24680724\end{array}$ \\
\hline$L P P$ & rs 16863233 & $\begin{array}{l}\text { acetaminophen, aflatoxin B1, benzo(a)pyrene, } \\
\text { estradiol, copper sulfate, methylmercuric chloride, } \\
\text { PCBs, tetrachlorodibenzodioxin, dibutyl phthalate }\end{array}$ & $\begin{array}{c}21420995,20106945,20064835, \\
19549813,23179753,21334430, \\
21632981,21266533\end{array}$ \\
\hline ZNF782 & rs 7037100 & arsenic, methylmercuric chloride & 21291286,23103053 \\
\hline OSGIN1 & rs62640905 & $\begin{array}{l}\text { acetaminophen, benzo(a)pyrene, bisphenol A, } \\
\text { copper sulfate, hydrogen peroxide, methylmercury } \\
\text { compounds, particulate matter, testosterone, } \\
\text { tetrachlorodibenzodioxin, vehicle emissions, } \\
\text { aflatoxin B1, cadmium chloride, carbon } \\
\text { tetrachloride, diethylhexyl phthalate, furan, } \\
\text { mercuric chloride, propiconazole }\end{array}$ & $\begin{array}{l}21420995,22316170,20170705, \\
19549813,23410634,22129749, \\
20307321,21592394,21632981, \\
20307321,19770486,20061341, \\
17484886,19850644,24183702, \\
16507785,21278054,17963808\end{array}$ \\
\hline MTG2 & rs35693261 & $\begin{array}{c}\text { arsenic, hydrogen peroxide, PCBs, dibutyl } \\
\text { phthalate, tetrachlorodibenzodioxin }\end{array}$ & $\begin{array}{c}18414638,18951874,19114083 \\
21266533,21570461\end{array}$ \\
\hline$T N R$ & rs 7539097 & copper sulfate, lead, PCBs, arsenic, chlorpyrifos & $\begin{array}{c}19549813,19921347,19114083, \\
24019935,18668222\end{array}$ \\
\hline
\end{tabular}


Appendix Table 11 continued....

\begin{tabular}{|c|c|c|c|}
\hline Gene/region & SNP & Chemical interaction & PubMed IDs \\
\hline ENPP1 & rs9493135 & $\begin{array}{l}\text { aflatoxin B1, benzo(a)pyrene diol epoxide, } \\
\text { mercury, potassium dichromate, progesterone, } \\
\text { tetrachlorodibenzodioxin, PCB, bisphenol A, } \\
\text { carbon tetrachloride, chlorpyrifos, dibutyl phthalate, } \\
\text { lipopolysaccharide, progesterone }\end{array}$ & $\begin{array}{l}22100608,20382639,17547211, \\
17547211,20864642,21632981, \\
24935251,24383497,23196670, \\
20739668,17484886,21356183, \\
21266533,17573159,23018184\end{array}$ \\
\hline CTGF & rs9493135 & $\begin{array}{l}\text { 1-nitropyrene, acetaminophen, arsenic, asbestos, } \\
\text { benzo(a)pyrene, benzo(a)pyrene diol epoxide, } \\
\text { estradiol, bisphenol A, cadmium, copper sulfate, } \\
\text { cycloheximide, diuron, dust, ethinyl estradiol, } \\
\text { fonofos, formaldehyde, hydrogen peroxide, } \\
\text { lipopolysaccharides, methylmercuric chloride, } \\
\text { parathion, piroxicam, smoke, sodium arsenite, } \\
\text { terbufos, tetrachlorodibenzodioxin, trichostatin A, } \\
\text { aflatoxin B1, aspirin, carbon tetrachloride, } \\
\text { chlorpyrifos, dibutyl phthalate, diuron, ethanol, } \\
\text { nitrofen, particulate matter, progesterone, } \\
\text { testosterone, tobacco smoke pollution, vehicle } \\
\text { emissions }\end{array}$ & $\begin{array}{c}19041380,22230336,24675094, \\
22076105,21632981,20018196, \\
14699072,20170705,19369054, \\
19549813,19684285,24172598, \\
15735009,20170705,22847954, \\
23416264,17965732,23179753, \\
22847954,23155386,12377979, \\
22847954,22298810,19606018, \\
24935251,19770486,14586741, \\
19484750,24586524,15240111, \\
20371981,14724832,21356183, \\
21266533,21551480,19280452, \\
21258935,23358140,21708214, \\
23018184,15788153,22761892, \\
21258935,19165385\end{array}$ \\
\hline ZNF540 & rs 16973890 & acetaminophen & 22230336 \\
\hline ZNF571 & rs 16973890 & formaldehyde & 20655997 \\
\hline$M F F$ & rs6722598 & acetaminophen, copper sulfate, hydrogen peroxide & $21420995,19549813,20044591$ \\
\hline PSME4 & rs1049894 & $\begin{array}{c}\text { arsenic trioxide, benzene, benzo(a)pyrene, estradiol, } \\
\text { copper sulfate, decamethrin, formaldehyde, } \\
\text { cadmium chloride, dibutyl phthalate, lead nitrate, } \\
\text { tetrachlorodibenzodioxin }\end{array}$ & $\begin{array}{c}22521957,19162166,23892563, \\
23019147,19549813,23892563, \\
19187980,21266533,19187980, \\
21570461\end{array}$ \\
\hline ACYP2 & rs11904365 & $\begin{array}{c}\text { atrazine, benzo(a)pyrene, estradiol, ethinyl } \\
\text { estradiol, copper sulfate, aflatoxin B1, arsenite, } \\
\text { bisphenol A, lead acetate, progesterone, } \\
\text { tetrachlorodibenzodioxin }\end{array}$ & $\begin{array}{l}22378314,22316170,23019147, \\
19549813,17942748,24935251, \\
19770486,18929588,21932408, \\
20542052,23012394,21570461\end{array}$ \\
\hline
\end{tabular}


Appendix Table 11 continued....

\begin{tabular}{|c|c|c|c|}
\hline Gene/region & SNP & Chemical interaction & PubMed IDs \\
\hline$R A B 28$ & $\begin{array}{l}\text { rs10003958 } \\
\text { rs9993832 }\end{array}$ & $\begin{array}{l}\text { benzo(a)pyrene, benzo(a)pyrene diol epoxide, } \\
\text { copper sulfate, potassium chromate(VI), dibutyl } \\
\text { phthalate, ethinyl estradiol, methylmercuric } \\
\text { chloride, tetrachlorodibenzodioxin }\end{array}$ & $\begin{array}{c}22316170,19150397,19549813, \\
22079256,23179753,21266533, \\
17942748,23458150,21570461, \\
15901671\end{array}$ \\
\hline CROCC & rs78888579 & $\begin{array}{l}\text { PCBs, benzo(a)pyrene, dibutyl phthalate, mercuric } \\
\text { chloride, tetrachlorodibenzodioxin }\end{array}$ & $\begin{array}{c}21334430,22228805,21266533, \\
16507785,21570461\end{array}$ \\
\hline KCNT2 & rs 10218650 & $\begin{array}{l}\text { acetaminophen, air pollutants, arsenite, } \\
\text { progesterone, benzo(a)pyrene, ethinyl estradiol }\end{array}$ & $\begin{array}{l}21420995,21757418,23974009, \\
21795739,19770486,17557909\end{array}$ \\
\hline GCFC2 & rs 10194197 & manganese, fipronil, potassium dichromate & $17175027,23962444,23608068$ \\
\hline CCDC173 & rs61741435 & tetrachlorodibenzodioxin & 22298810 \\
\hline CADM2 & rs4856270 & $\begin{array}{c}\text { aflatoxin B1, methylmercuric chloride, } \\
\text { tetrachlorodibenzodioxin }\end{array}$ & $20106945,23179753,20055451$ \\
\hline KIAA0922 & rs2165084 & $\begin{array}{l}\text { air pollutants, atrazine, benzo(a)pyrene diol } \\
\text { epoxide, } \mathrm{PCB} \text {, potassium chromate(VI) }\end{array}$ & $\begin{array}{c}21757418,22378314,20382639 \\
21334430,22079256\end{array}$ \\
\hline TRERF1 & rs11751765 & $\begin{array}{c}\text { aflatoxin B1, arsenic trioxide, asbestos, estradiol, } \\
\text { potassium chromate(VI), Progesterone, ethinyl } \\
\text { estradiol, furan, tetrachlorodibenzodioxin, vehicle } \\
\text { emissions }\end{array}$ & $\begin{array}{l}22100608,20458559,17331233, \\
20660070,22079256,20660070, \\
24935251,24935251,17942748, \\
22079235,17942748,23940539\end{array}$ \\
\hline$M E O X 2$ & rs7797334 & $\begin{array}{l}\text { acetaminophen, environmental pollutants, mercury, } \\
\text { tetrachlorodibenzodioxin, PCB, chlorine, dibutyl } \\
\text { phthalate, diethylstilbestrol, nickel, ozone }\end{array}$ & $\begin{array}{l}22230336,12655031,16823088, \\
22298810,21934132,20959002, \\
18636392,21266533,15591538, \\
23153324,12540486,18636392,\end{array}$ \\
\hline$I S P D$ & rs7797334 & $\begin{array}{l}\text { PCB, aflatoxin B1, benzo(a)pyrene, copper sulfate, } \\
\text { diquat, potassium dichromate }\end{array}$ & $\begin{array}{l}19114083,19770486,19770486, \\
19789285,22814884,23608068\end{array}$ \\
\hline CDC14C & rs4278110 & estradiol & 22574217 \\
\hline COLEC10 & rs11987106 & arsenic trioxide, acetaminophen, benzo(a)pyrene & $15761015,17562736,21569818$ \\
\hline CCIN & rs61735202 & $\begin{array}{c}\text { acetaminophen, cadmium chloride, } \\
\text { tetrachlorodibenzodioxin } \\
\end{array}$ & $17562736,22457795,21354282$ \\
\hline
\end{tabular}




\begin{tabular}{|c|c|c|c|}
\hline Gene/region & SNP & Chemical interaction & PubMed IDs \\
\hline MGMT & rs943811 & $\begin{array}{l}\text { aflatoxin B1, benzo(a)pyrene, ethylene dibromide, } \\
\text { dust, fonofos, parathion, polycyclic aromatic } \\
\text { hydrocarbon, acetaminophen, ammonium chloride, } \\
\text { arsenite, estradiol, bisphenol A, carbon } \\
\text { tetrachloride, diethylstilbestrol, furan, } \\
\text { methylnitrosourea, ozone, piperonyl butoxide, } \\
\text { praziquantel, sodium arsenite, } \\
\text { tetrachlorodibenzodioxin } \\
\end{array}$ & $\begin{array}{c}20106945,20064835,12151404, \\
17805423,8823159,22847954, \\
23543013,12883083,16483693, \\
18929588,20965277,20965277, \\
17484886,15890375,22507866, \\
20965277,23358140,18636392, \\
15890375,21813463,11241755, \\
21215274,17292431 \\
\end{array}$ \\
\hline TMEM117 & rs11182332 & $\begin{array}{l}\text { aflatoxin B1, benzo(a)pyrene, benzo(a)pyrene diol } \\
\text { epoxide, copper sulfate, diuron, potassium } \\
\text { chromate(VI), PCB }\end{array}$ & $\begin{array}{c}\text { 20106945, 21714911, 19150397, } \\
\text { 19549813, 24172598, 22079256, } \\
23724009,24935251,23179753, \\
19114083\end{array}$ \\
\hline PARP4 & rs4770684 & $\begin{array}{l}\text { acetaminophen, arsenite, estradiol, copper sulfate, } \\
\text { hydrogen peroxide, nicotine, potassium } \\
\text { chromate(VI), aflatoxin B1, arsenic, benzo(a)pyrene }\end{array}$ & $\begin{array}{c}21420995,23974009,22574217, \\
19549813,21179406,23825647, \\
22079256,19770486,19654921, \\
21839799\end{array}$ \\
\hline UBE3A & rs7182827 & $\begin{array}{l}\text { arsenic trioxide, atrazine, benzo(a)pyrene diol, } \\
\text { estradiol, nicotine, acetaminophen, benzo(a)pyrene, } \\
\text { bisphenol A, cadmium sulfate, } \\
\text { tetrachlorodibenzodioxin, tobacco smoke pollution }\end{array}$ & $\begin{array}{l}20458559,22378314,19150397, \\
23665804,12588870,16140955, \\
17562736,22228805,23593014, \\
16221973,21570461,18087596\end{array}$ \\
\hline KRTAP21-1 & rs7275217 & aluminum & 21298039 \\
\hline RGSL1 & rs41299607 & cadmium & 24376830 \\
\hline LMO2 & rs9282777 & $\begin{array}{l}\text { arsenic trioxide, Benzo(a)pyrene, Estradiol, } \\
\text { Isotretinoin, Testosterone, } \\
\text { Tetrachlorodibenzodioxin, Tretinoin, trichostatin A, } \\
\text { Valproic Acid, bisphenol A, Cadmium Chloride, } \\
\text { Carbon Tetrachloride, Dibutyl Phthalate, Ozone }\end{array}$ & $\begin{array}{c}19128835,22316170,22574217, \\
20436886,21592394,22903824, \\
21934132,24935251,24935251, \\
21786754,22457795,17484886, \\
21266533,17095637\end{array}$ \\
\hline CTNNA2 & rs 10520291 & $\begin{array}{l}\text { acetaminophen, estradiol, progesterone, } \\
\text { testosterone, 2,6-dinitrotoluene, bisphenol A, } \\
\text { dibutyl phthalate, dioxins, ethinyl estradiol, ozone, } \\
\text { tetrachlorodibenzodioxin }\end{array}$ & $\begin{array}{l}\text { 22230336, 20660070, 20660070, } \\
21592394,23179753,20406850, \\
21786754,21266533,20463971, \\
17942748,12763052,21570461, \\
16211290\end{array}$ \\
\hline
\end{tabular}


Appendix Table 11 continued....

\begin{tabular}{|c|c|c|c|}
\hline Gene/region & SNP & Chemical interaction & PubMed IDs \\
\hline PDE4B & rs12037891 & $\begin{array}{l}\text { arsenic, arsenic trioxide, benzo(a)pyrene, estradiol, } \\
\text { formaldehyde, lipopolysaccharides, progesterone, } \\
\text { smoke, testosterone, atrazine, bisphenol A, carbon } \\
\text { tetrachloride, dibutyl phthalate, fipronil, furan, } \\
\text { testosterone, tetrachlorodibenzodioxin }\end{array}$ & $\begin{array}{c}16835338,15761015,22316170, \\
14699072,23649840,20655997, \\
21854221,21795739,21095227, \\
18535249,19541795,21932408, \\
17484886,21266533,22079235, \\
18535249,22298810\end{array}$ \\
\hline CCHCR1 & rs 130069 & $\begin{array}{l}\text { acetaminophen, potassium chromate(VI), } \\
\text { benzo(a)pyrene, tetrachlorodibenzodioxin }\end{array}$ & $22230336,22228805,21570461$ \\
\hline TCF19 & rs6929434 & $\begin{array}{l}\text { acetaminophen, aflatoxin B1, benzo(a)pyrene diol, } \\
\text { estradiol, copper sulfate, formaldehyde, testosterone }\end{array}$ & $\begin{array}{c}2230336,20106945,20382639, \\
19167446,19549813,23649840, \\
23649840,21592394 \\
\end{array}$ \\
\hline KDM4C & rs10975823 & $\begin{array}{l}\text { acetaminophen, aflatoxin B1, atrazine, } \\
\text { benzo(a)pyrene, potassium chromate(VI) }\end{array}$ & $\begin{array}{c}22230336,22378314,22378314 \\
22316170,22079256\end{array}$ \\
\hline ZNF782 & $\begin{array}{l}\text { rs4645656 } \\
\text { rs7848710 } \\
\text { rs6477733 }\end{array}$ & arsenic, methylmercuric chloride & 21291286,23103053 \\
\hline SUMF1 & rs2633820 & $\begin{array}{c}\text { copper sulfate, formaldehyde, hydrogen peroxide, } \\
\text { tetrachlorodibenzodioxin, bisphenol A, dibutyl } \\
\text { phthalate, tetrachlorodibenzodioxin }\end{array}$ & $\begin{array}{c}19549813,23649840,18951874, \\
22574217,24935251,21786754, \\
21266533,21570461\end{array}$ \\
\hline NEDD9 & rs9469223 & $\begin{array}{l}\text { benzene, benzo(a)pyrene, benzo(a)pyrene diol, } \\
\text { estradiol, bisphenol A, copper sulfate, diuron, } \\
\text { ethylbenzene, formaldehyde, methylmercuric } \\
\text { chloride, particulate matter, PCB, smoke, } \\
\text { tetrachlorodibenzodioxin, tobacco tar, toluene, 2,6- } \\
\text { dinitrotoluene, acetaminophen, carbon tetrachloride, } \\
\text { dibutyl phthalate, diethylstilbestrol, diuron, ethinyl } \\
\text { estradiol, lipopolysaccharide, nicotine, } \\
\text { propiconazole, sodium arsenite }\end{array}$ & $\begin{array}{l}20359517,20064835,20382639, \\
22574217,20170705,19549813, \\
24172598,20359517,23649840, \\
23179753,22178795,16474171, \\
21095227,19619570,19559774, \\
24383497,20406850,18700144, \\
17484886,21266533,21658437, \\
24172598,20170705,18930950, \\
17456735,16014739,19015723\end{array}$ \\
\hline
\end{tabular}

$\mathrm{SNP}=$ single nucleotide polymorphism 
Appendix Table 12: Top one hundred SNPs associated with childhood ALL risk with age-specificity, results based on the lowest $P$ values

\begin{tabular}{|c|c|c|c|c|c|c|c|c|c|}
\hline \multirow[b]{2}{*}{ SNP } & \multirow[b]{2}{*}{ Gene/region } & \multirow[b]{2}{*}{ Chr } & \multirow[b]{2}{*}{$\begin{array}{l}\text { Minor } \\
\text { allele }\end{array}$} & \multirow[b]{2}{*}{ Feature } & \multicolumn{2}{|c|}{$\begin{array}{l}\text { Minor allele } \\
\text { frequency }\end{array}$} & \multirow[b]{2}{*}{$\mathrm{OR}_{\text {allele }}(95 \% \mathrm{CI})^{*}$} & \multirow[b]{2}{*}{$P$ value** } & \multirow[b]{2}{*}{$\begin{array}{c}\text { P value } \\
\text { (permutation) }\end{array}$} \\
\hline & & & & & $1-5$ year & 6-10 year & & & \\
\hline rs10505918 & SOX5 & 12 & $\mathrm{~A}$ & intron & 0.42 & 0.19 & $3.15(1.90-5.24)$ & $9.84 \times 10^{-06}$ & $1 \times 10^{-4}$ \\
\hline rs11997355 & $8 \mathrm{q} 21.11$ & 8 & $\mathrm{~T}$ & intergenic & 0.05 & 0.19 & $0.22(0.11-0.44)$ & $2.74 \times 10^{-05}$ & $1 \times 10^{-4}$ \\
\hline rs2388773 & $16 \mathrm{q} 12.2$ & 16 & $\mathrm{C}$ & intergenic & 0.14 & 0.35 & $0.35(0.21-0.57)$ & $3.78 \times 10^{-05}$ & $1 \times 10^{-4}$ \\
\hline rs665159 & CHRM3 & 1 & $\mathrm{~T}$ & intron & 0.55 & 0.33 & $2.46(1.58-3.84)$ & $7.42 \times 10^{-05}$ & $2 \times 10^{-4}$ \\
\hline rs3954950 & $9 \mathrm{p} 24.2$ & 9 & $\mathrm{~T}$ & intergenic & 0.24 & 0.43 & $0.38(0.24-0.62)$ & $7.77 \times 10^{-05}$ & $1 \times 10^{-4}$ \\
\hline rs7818844 & $8 \mathrm{p} 12$ & 8 & $\mathrm{~T}$ & intergenic & 0.4 & 0.6 & $0.38(0.23-0.61)$ & $7.86 \times 10^{-05}$ & $1 \times 10^{-4}$ \\
\hline rs6972158 & NPSR1 & 7 & G & intron & 0.34 & 0.14 & $3.03(1.74-5.25)$ & $8.30 \times 10^{-05}$ & $1 \times 10^{-4}$ \\
\hline rs7320982 & $8 \mathrm{p} 12$ & 13 & $\mathrm{C}$ & intergenic & 0.19 & 0.38 & $0.39(0.24-0.62)$ & $8.33 \times 10^{-05}$ & $1 \times 10^{-4}$ \\
\hline rs7583258 & $2 \mathrm{q} 22.3$ & 2 & G & intergenic & 0.09 & 0.24 & $0.29(0.16-0.54)$ & $8.79 \times 10^{-05}$ & $2 \times 10^{-4}$ \\
\hline rs1882591 & $16 \mathrm{q} 12.2$ & 16 & G & intergenic & 0.22 & 0.42 & $0.42(0.27-0.64)$ & $9.60 \times 10^{-05}$ & $2 \times 10^{-4}$ \\
\hline rs7318021 & $13 \mathrm{q} 31.3$ & 13 & $\mathrm{~T}$ & intergenic & 0.23 & 0.43 & $0.42(0.27-0.66)$ & $1.16 \times 10^{-04}$ & $1 \times 10^{-4}$ \\
\hline rs7016929 & $8 \mathrm{q} 31.3$ & 8 & A & intergenic & 0.2 & 0.39 & $0.40(0.25-0.64)$ & $1.18 \times 10^{-04}$ & $1 \times 10^{-4}$ \\
\hline rs10079494 & $5 q 14.1$ & 5 & A & intergenic & 0.28 & 0.1 & $3.39(1.82-6.32)$ & $1.22 \times 10^{-04}$ & $2 \times 10^{-4}$ \\
\hline rs10250709 & NPSR1 & 7 & $\bar{A}$ & intron & 0.33 & 0.14 & $3.03(1.72-5.32)$ & $1.22 \times 10^{-04}$ & $1 \times 10^{-4}$ \\
\hline rs7720650 & $5 q 31.2$ & 5 & A & intergenic & 0.12 & 0.27 & $0.32(0.18-0.57)$ & $1.33 \times 10^{-04}$ & $1 \times 10^{-4}$ \\
\hline rs2949827 & $7 q 31.1$ & 7 & A & intergenic & 0.08 & 0.21 & $0.29(0.16-0.55)$ & $1.44 \times 10^{-04}$ & $2 \times 10^{-4}$ \\
\hline rs2396653 & $7 q 31.1$ & 7 & A & intergenic & 0.14 & 0.29 & $0.34(0.19-0.59)$ & $1.57 \times 10^{-04}$ & $2 \times 10^{-4}$ \\
\hline rs202050948 & CD97 & 19 & $\mathrm{~T}$ & intron & 0.04 & 0.15 & $0.22(0.10-0.48)$ & $1.72 \times 10^{-04}$ & $1 \times 10^{-4}$ \\
\hline rs1191818 & $2 \mathrm{p} 24.1$ & 2 & $\mathrm{C}$ & intergenic & 0.38 & 0.59 & $0.43(0.28-0.67)$ & $1.76 \times 10^{-04}$ & $6 \times 10^{-4}$ \\
\hline rs3800292 & GNMT & 6 & G & intron & 0.02 & 0.11 & $0.13(0.04-0.38)$ & $1.87 \times 10^{-04}$ & $1 \times 10^{-4}$ \\
\hline
\end{tabular}


Appendix Table 12 continued....

\begin{tabular}{|c|c|c|c|c|c|c|c|c|c|}
\hline \multirow[b]{2}{*}{ SNP } & \multirow[b]{2}{*}{ Gene/region } & \multirow[b]{2}{*}{ Chr } & \multirow[b]{2}{*}{$\begin{array}{l}\text { Minor } \\
\text { allele }\end{array}$} & \multirow[b]{2}{*}{ Feature } & \multicolumn{2}{|c|}{$\begin{array}{c}\text { Minor allele } \\
\text { frequency }\end{array}$} & \multirow[b]{2}{*}{$\mathrm{OR}_{\text {allele }}(95 \% \mathrm{CI})^{*}$} & \multirow[b]{2}{*}{$P$ value** } & \multirow[b]{2}{*}{$\begin{array}{c}\text { P value } \\
\text { (permutation) }^{\dagger \dagger}\end{array}$} \\
\hline & & & & & $1-5$ year & 6-10 year & & & \\
\hline rs1881116 & C7orf50 & 7 & A & intron & 0.38 & 0.21 & $2.70(1.60-4.57)$ & $2.04 \times 10^{-04}$ & $2 \times 10^{-4}$ \\
\hline rs4821717 & $22 \mathrm{q} 13.1$ & 22 & A & intergenic & 0.45 & 0.26 & $2.46(1.53-3.97)$ & $2.06 \times 10^{-04}$ & $5 \times 10^{-4}$ \\
\hline rs2212824 & $21 \mathrm{q} 21.3$ & 21 & $\bar{G}$ & intergenic & 0.44 & 0.26 & $2.52(1.55-4.11)$ & $2.15 \times 10^{-04}$ & $2 \times 10^{-4}$ \\
\hline rs7157104 & $14 \mathrm{q} 13.2$ & 14 & $\mathrm{G}$ & intergenic & 0.15 & 0.3 & $0.36(0.21-0.62)$ & $2.28 \times 10^{-04}$ & $1 \times 10^{-4}$ \\
\hline rs2051713 & $12 \mathrm{q} 21.33$ & 12 & G & intergenic & 0.52 & 0.32 & $2.40(1.51-3.82)$ & $2.30 \times 10^{-04}$ & $2 \times 10^{-4}$ \\
\hline rs2125649 & $8 \mathrm{p} 21.3$ & 8 & G & intergenic & 0.07 & 0.19 & $0.28(0.14-0.55)$ & $2.32 \times 10^{-04}$ & $1 \times 10^{-4}$ \\
\hline rs6739633 & $2 \mathrm{p} 24.3$ & 2 & $\mathrm{~T}$ & intergenic & 0.48 & 0.28 & $2.36(1.50-3.74)$ & $2.33 \times 10^{-04}$ & $2 \times 10^{-4}$ \\
\hline rs738027 & PYROXD1 & 12 & A & intron & 0.43 & 0.24 & $2.45(1.52-3.96)$ & $2.55 \times 10^{-04}$ & $3 \times 10^{-4}$ \\
\hline rs237320 & $17 \mathrm{p} 12$ & 17 & $\mathrm{~T}$ & intergenic & 0.11 & 0.26 & $0.35(0.20-0.62)$ & $2.65 \times 10^{-04}$ & $1 \times 10^{-4}$ \\
\hline rs139886 & SOX10 & 22 & $\mathrm{C}$ & intron & 0.47 & 0.28 & $2.41(1.50-3.87)$ & $2.67 \times 10^{-04}$ & $2 \times 10^{-4}$ \\
\hline rs13116233 & $4 q 34.3$ & 4 & $\mathrm{C}$ & intergenic & 0.36 & 0.17 & $2.56(1.54-4.25)$ & $2.69 \times 10^{-04}$ & $3 \times 10^{-4}$ \\
\hline rs6519091 & MICALL1 & 22 & $\bar{C}$ & intron & 0.51 & 0.32 & $2.33(1.48-3.69)$ & $2.87 \times 10^{-04}$ & $5 \times 10^{-4}$ \\
\hline rs6109487 & 20p12.1 & 20 & A & intergenic & 0.24 & 0.09 & $3.55(1.79-7.03)$ & $2.90 \times 10^{-04}$ & $3 \times 10^{-4}$ \\
\hline rs1151277 & $16 \mathrm{q} 12.2$ & 16 & $\mathrm{~T}$ & intergenic & 0.13 & 0.29 & $0.38(0.23-0.64)$ & $2.93 \times 10^{-04}$ & $1 \times 10^{-4}$ \\
\hline rs6052937 & SLC23A2 & 20 & A & 3'UTR & 0.12 & 0.27 & $0.38(0.22-0.64)$ & $2.99 \times 10^{-04}$ & $1 \times 10^{-4}$ \\
\hline rs34099167 & NEK1 & 4 & $\mathrm{C}$ & coding & 0.04 & 0.15 & $0.24(0.11-0.52)$ & $3.07 \times 10^{-04}$ & $1 \times 10^{-4}$ \\
\hline rs7722067 & $5 \mathrm{q} 14.2$ & 5 & $\mathrm{C}$ & intergenic & 0.16 & 0.31 & $0.38(0.22-0.64)$ & $3.08 \times 10^{-04}$ & $1 \times 10^{-4}$ \\
\hline rs1004327 & CLNK & 4 & $\mathrm{C}$ & intron & 0.41 & 0.24 & $2.54(1.53-4.22)$ & $3.11 \times 10^{-04}$ & $3 \times 10^{-4}$ \\
\hline rs3993401 & $12 \mathrm{q} 21.31$ & 12 & $\mathrm{C}$ & intergenic & 0.24 & 0.41 & $0.41(0.25-0.67)$ & $3.16 \times 10^{-04}$ & $3 \times 10^{-4}$ \\
\hline rs6492498 & $13 \mathrm{q} 31.3$ & 13 & $\mathrm{~A}$ & intergenic & 0.29 & 0.49 & $0.45(0.30-0.70)$ & $3.22 \times 10^{-04}$ & $5.9 \times 10^{-4}$ \\
\hline rs2806907 & $13 q 14.13$ & 13 & $\mathrm{~T}$ & intergenic & 0.32 & 0.51 & $0.44(0.28-0.69)$ & $3.29 \times 10^{-04}$ & $4 \times 10^{-4}$ \\
\hline
\end{tabular}


Appendix Table 12 continued....

\begin{tabular}{|c|c|c|c|c|c|c|c|c|c|}
\hline \multirow[b]{2}{*}{ SNP } & \multirow[b]{2}{*}{ Gene/region } & \multirow[b]{2}{*}{ Chr } & \multirow[b]{2}{*}{$\begin{array}{l}\text { Minor } \\
\text { allele }\end{array}$} & \multirow[b]{2}{*}{ Feature } & \multicolumn{2}{|c|}{$\begin{array}{c}\text { Minor allele } \\
\text { frequency }\end{array}$} & \multirow[b]{2}{*}{$\mathrm{OR}_{\text {allele }}(95 \% \mathrm{CI})^{*}$} & \multirow[b]{2}{*}{$P$ value** } & \multirow[b]{2}{*}{$\begin{array}{c}\text { P value } \\
\text { (permutation) })^{\dagger \dagger}\end{array}$} \\
\hline & & & & & 1-5 year & 6-10 year & & & \\
\hline rs2101919 & NID2 & 14 & $\mathrm{G}$ & coding & 0.28 & 0.46 & $0.44(0.28-0.69)$ & $3.31 \times 10^{-04}$ & $4 \times 10^{-4}$ \\
\hline rs2899293 & ANKRD54 & 22 & $\mathrm{G}$ & intron & 0.51 & 0.32 & $2.32(1.47-3.68)$ & $3.32 \times 10^{-04}$ & $5.9 \times 10^{-4}$ \\
\hline rs1028695 & SPATA13 & 13 & $\mathrm{~T}$ & intron & 0.14 & 0.29 & $0.38(0.23-0.65)$ & $3.38 \times 10^{-04}$ & $2 \times 10^{-4}$ \\
\hline rs2185230 & PCSK5 & 9 & $\mathrm{~A}$ & intron & 0.34 & 0.17 & $2.76(1.58-4.81)$ & $3.39 \times 10^{-04}$ & $3 \times 10^{-4}$ \\
\hline rs4254428 & ZNF420 & 19 & $\mathrm{C}$ & intron & 0.43 & 0.63 & $0.45(0.29-0.70)$ & $3.41 \times 10^{-04}$ & $4 \times 10^{-4}$ \\
\hline rs139866 & POLR2F & 22 & $\mathrm{C}$ & intron & 0.45 & 0.27 & $2.40(1.49-3.88)$ & $3.46 \times 10^{-04}$ & $3 \times 10^{-4}$ \\
\hline rs5028798 & $11 \mathrm{p} 13$ & 11 & $\mathrm{~T}$ & intergenic & 0.28 & 0.46 & $0.44(0.28-0.69)$ & $3.48 \times 10^{-04}$ & $5 \times 10^{-4}$ \\
\hline rs2065212 & $13 \mathrm{q} 31.3$ & 13 & $\mathrm{~A}$ & intergenic & 0.31 & 0.51 & $0.46(0.30-0.71)$ & $3.51 \times 10^{-04}$ & $5 \times 10^{-4}$ \\
\hline rs222314 & $7 \mathrm{p} 21.3$ & 7 & $\mathrm{G}$ & intergenic & 0.2 & 0.05 & $4.52(1.98-10.35)$ & $3.52 \times 10^{-04}$ & $2 \times 10^{-4}$ \\
\hline rs1894381 & TMC5 & 16 & G & intron & 0.16 & 0.32 & $0.41(0.25-0.67)$ & $3.52 \times 10^{-04}$ & $5 \times 10^{-4}$ \\
\hline rs9809647 & $3 q 28$ & 3 & $\mathrm{~T}$ & intergenic & 0.34 & 0.54 & $0.48(0.32-0.72)$ & $3.53 \times 10^{-04}$ & $2 \times 10^{-4}$ \\
\hline rs898982 & $1 \mathrm{p} 34.3$ & 1 & $\mathrm{C}$ & intergenic & 0.04 & 0.14 & $0.24(0.11-0.52)$ & $3.65 \times 10^{-04}$ & $3 \times 10^{-4}$ \\
\hline rs1868368 & $8 \mathrm{q} 24.23$ & 8 & $\mathrm{C}$ & intergenic & 0.19 & 0.35 & $0.41(0.25-0.67)$ & $3.74 \times 10^{-04}$ & $1 \times 10^{-4}$ \\
\hline rs9392857 & $6 \mathrm{p} 25.3$ & 6 & $\mathrm{~A}$ & intergenic & 0.3 & 0.48 & $0.44(0.28-0.69)$ & $3.77 \times 10^{-04}$ & $1 \times 10^{-4}$ \\
\hline rs8014810 & BRMS1L & 14 & $\mathrm{~T}$ & intron & 0.13 & 0.26 & $0.36(0.20-0.63)$ & $3.88 \times 10^{-04}$ & $2 \times 10^{-4}$ \\
\hline rs8021355 & RALGAPA1 & 14 & $\mathrm{~A}$ & intron & 0.15 & 0.29 & $0.38(0.22-0.65)$ & $4.10 \times 10^{-04}$ & $3 \times 10^{-4}$ \\
\hline rs7144565 & RALGAPA1 & 14 & $\mathrm{~A}$ & intron & 0.15 & 0.29 & $0.38(0.22-0.65)$ & $4.10 \times 10^{-04}$ & $3 \times 10^{-4}$ \\
\hline rs2274068 & RALGAPA1 & 14 & $\mathrm{C}$ & coding & 0.15 & 0.29 & $0.38(0.22-0.65)$ & $4.10 \times 10^{-04}$ & $3 \times 10^{-4}$ \\
\hline rs762562 & $\begin{array}{l}\text { ERCC1/ } \\
C D 3 E A P\end{array}$ & 19 & $\mathrm{G}$ & 3'UTR & 0.19 & 0.36 & $0.43(0.26-0.68)$ & $4.22 \times 10^{-04}$ & $2 \times 10^{-4}$ \\
\hline rs4948088 & $7 \mathrm{p} 12.1$ & 7 & $\mathrm{~A}$ & intergenic & 0.05 & 0.15 & $0.26(0.12-0.55)$ & $4.23 \times 10^{-04}$ & $2 \times 10^{-4}$ \\
\hline rs3850942 & MARK2 & 11 & $\mathrm{C}$ & intron & 0.03 & 0.12 & $0.20(0.09-0.49)$ & $4.35 \times 10^{-04}$ & $1 \times 10^{-4}$ \\
\hline
\end{tabular}


Appendix Table 12 continued....

\begin{tabular}{|c|c|c|c|c|c|c|c|c|c|}
\hline & & & & & $\begin{array}{l}\text { Min } \\
\text { fre }\end{array}$ & $\begin{array}{l}\text { allele } \\
\text { ency }\end{array}$ & & & \\
\hline SNP & Gene/region & Chr & $\begin{array}{l}\text { Minor } \\
\text { allele }\end{array}$ & Feature & 1-5 year & 6-10 year & $\mathrm{OR}_{\text {allele }}(95 \% \mathrm{CI})^{*}$ & $P$ value** & $\begin{array}{c}\text { P value } \\
\text { (permutation) }^{\dagger \dagger}\end{array}$ \\
\hline rs2899469 & $15 q 21.2$ & 15 & $\mathrm{G}$ & intergenic & 0.48 & 0.29 & $2.24(1.43-3.52)$ & $4.45 \times 10^{-04}$ & $4 \times 10^{-4}$ \\
\hline rs8011343 & MDGA2 & 14 & $\mathrm{C}$ & intron & 0.19 & 0.06 & $4.21(1.89-9.41)$ & $4.51 \times 10^{-04}$ & $5 \times 10^{-4}$ \\
\hline rs2031293 & $13 q 34$ & 13 & A & intergenic & 0.43 & 0.63 & $0.47(0.30-0.71)$ & $4.61 \times 10^{-04}$ & $3 \times 10^{-4}$ \\
\hline rs11171543 & $12 \mathrm{q} 12$ & 12 & $\mathrm{~A}$ & intergenic & 0.09 & 0.24 & $0.37(0.21-0.64)$ & $4.75 \times 10^{-04}$ & $5.9 \times 10^{-4}$ \\
\hline rs11172024 & KIF21A & 12 & $\mathrm{C}$ & intron & 0.06 & 0.18 & $0.30(0.15-0.59)$ & $4.76 \times 10^{-04}$ & $3 \times 10^{-4}$ \\
\hline rs1510136 & $4 q 31.21$ & 4 & $\mathrm{G}$ & intergenic & 0.19 & 0.35 & $0.44(0.28-0.70)$ & $4.80 \times 10^{-04}$ & $4 \times 10^{-4}$ \\
\hline rs16979806 & $21 \mathrm{q} 21.3$ & 21 & $\mathrm{~T}$ & intergenic & 0.21 & 0.38 & $0.45(0.29-0.71)$ & $4.86 \times 10^{-04}$ & $6.9 \times 10^{-4}$ \\
\hline rs17017488 & CCSER1 & 4 & $\mathrm{C}$ & intron & 0.36 & 0.54 & $0.47(0.31-0.72)$ & $5.04 \times 10^{-04}$ & $5.9 \times 10^{-4}$ \\
\hline rs10511980 & MAMDC2 & 9 & A & intron & 0.27 & 0.45 & $0.46(0.30-0.71)$ & $5.04 \times 10^{-04}$ & $5 \times 10^{-4}$ \\
\hline rs2620870 & $12 q 14.1$ & 12 & $\mathrm{G}$ & intergenic & 0.55 & 0.36 & $2.06(1.37-3.10)$ & $5.08 \times 10^{-04}$ & $4 \times 10^{-4}$ \\
\hline rs377621 & $21 \mathrm{q} 21.3$ & 21 & $\mathrm{G}$ & intergenic & 0.23 & 0.41 & $0.46(0.30-0.72)$ & $5.15 \times 10^{-04}$ & $7.9 \times 10^{-4}$ \\
\hline rs6510588 & ZNF420 & 19 & A & intron & 0.43 & 0.62 & $0.46(0.30-0.72)$ & $5.17 \times 10^{-04}$ & $5 \times 10^{-4}$ \\
\hline rs10739501 & $9 q 33.1$ & 9 & $\mathrm{C}$ & intergenic & 0.34 & 0.52 & $0.46(0.29-0.71)$ & $5.18 \times 10^{-04}$ & $5.9 \times 10^{-4}$ \\
\hline rs6834625 & $4 q 31.21$ & 4 & $\mathrm{G}$ & intergenic & 0.17 & 0.33 & $0.44(0.28-0.70)$ & $5.22 \times 10^{-04}$ & $3 \times 10^{-4}$ \\
\hline rs1209145 & $14 q 22.3$ & 14 & $\mathrm{G}$ & intergenic & 0.17 & 0.05 & $4.48(1.91-10.5)$ & $5.51 \times 10^{-04}$ & $2 \times 10^{-4}$ \\
\hline rs1481046 & $8 \mathrm{q} 13.3$ & 8 & $\mathrm{G}$ & intergenic & 0.3 & 0.15 & $2.71(1.54-4.77)$ & $5.57 \times 10^{-04}$ & $4 \times 10^{-4}$ \\
\hline rs924615 & $5 q 14.2$ & 5 & $\mathrm{~T}$ & intergenic & 0.26 & 0.43 & $0.47(0.31-0.72)$ & $5.70 \times 10^{-04}$ & $1 \times 10^{-4}$ \\
\hline rs26784 & MSH3 & 5 & $\mathrm{C}$ & intron & 0.43 & 0.26 & $2.28(1.43-3.65)$ & $5.71 \times 10^{-04}$ & $5.9 \times 10^{-4}$ \\
\hline rs11120342 & PTPN14 & 1 & $\mathrm{C}$ & intron & 0.2 & 0.36 & $0.45(0.28-0.71)$ & $5.76 \times 10^{-04}$ & $8.9 \times 10^{-4}$ \\
\hline rs2830698 & $21 \mathrm{q} 21.3$ & 21 & $\mathrm{~T}$ & intergenic & 0.2 & 0.37 & $0.44(0.27-0.70)$ & $5.77 \times 10^{-04}$ & $2 \times 10^{-4}$ \\
\hline rs6037033 & $20 p 11.21$ & 20 & A & intergenic & 0.32 & 0.49 & $0.45(0.29-0.71)$ & $5.80 \times 10^{-04}$ & $5.9 \times 10^{-4}$ \\
\hline
\end{tabular}


Appendix Table 12 continued....

\begin{tabular}{|c|c|c|c|c|c|c|c|c|c|}
\hline & & & & & $\begin{array}{l}\text { Minc } \\
\text { freg }\end{array}$ & $\begin{array}{l}\text { allele } \\
\text { ency }\end{array}$ & & & \\
\hline SNP & Gene/region & Chr & $\begin{array}{l}\text { Minor } \\
\text { allele }\end{array}$ & Feature & 1-5 year & 6-10 year & $\mathrm{OR}_{\text {allele }}(95 \% \mathrm{CI}) *$ & $P$ value** & $\begin{array}{c}\text { P value } \\
(\text { permutation) }\end{array}$ \\
\hline rs12073211 & $1 \mathrm{p} 32.1$ & 1 & $\mathrm{C}$ & intergenic & 0.35 & 0.53 & $0.46(0.30-0.72)$ & $5.82 \times 10^{-04}$ & $4 \times 10^{-4}$ \\
\hline rs3825594 & NID2 & 14 & $\mathrm{~T}$ & intron & 0.26 & 0.43 & $0.46(0.29-0.72)$ & $5.86 \times 10^{-04}$ & $5 \times 10^{-4}$ \\
\hline rs742390 & $1 \mathrm{p} 36.11$ & 1 & $\mathrm{~T}$ & intron & 0.38 & 0.21 & $2.43(1.46-4.02)$ & $5.87 \times 10^{-04}$ & $5.9 \times 10^{-4}$ \\
\hline rs973358 & SHISA6 & 17 & A & intron & 0.46 & 0.29 & $2.32(1.44-3.75)$ & $5.92 \times 10^{-04}$ & $5.9 \times 10^{-4}$ \\
\hline rs10133677 & MDGA2 & 14 & G & intron & 0.16 & 0.05 & $4.92(1.98-12.23)$ & $5.97 \times 10^{-04}$ & $3 \times 10^{-4}$ \\
\hline rs1493897 & $16 q 12.2$ & 16 & A & intergenic & 0.17 & 0.33 & $0.44(0.27-0.70)$ & $5.99 \times 10^{-04}$ & $6.9 \times 10^{-4}$ \\
\hline rs9694342 & AGO2 & 8 & $\mathrm{~T}$ & intron & 0.24 & 0.41 & $0.46(0.30-0.72)$ & $6.17 \times 10^{-04}$ & $5.9 \times 10^{-4}$ \\
\hline rs7952853 & OR6C2 & 12 & $\mathrm{~T}$ & intron & 0.28 & 0.46 & $0.45(0.29-0.71)$ & $6.20 \times 10^{-04}$ & $3 \times 10^{-4}$ \\
\hline rs6943934 & $7 \mathrm{p} 22.3$ & 7 & $\mathrm{C}$ & intergenic & 0.04 & 0.14 & $0.25(0.11-0.56)$ & $6.21 \times 10^{-04}$ & $1 \times 10^{-4}$ \\
\hline rs1610315 & $6 q 27$ & 6 & $\mathrm{C}$ & intergenic & 0.21 & 0.38 & $0.43(0.27-0.70)$ & $6.24 \times 10^{-04}$ & $4 \times 10^{-4}$ \\
\hline rs1521244 & $2 \mathrm{p} 24.3$ & 2 & $\mathrm{C}$ & intergenic & 0.43 & 0.26 & $2.26(1.42-3.61)$ & $6.26 \times 10^{-04}$ & $4 \times 10^{-4}$ \\
\hline rs9897794 & EFCAB5 & 17 & $\mathrm{~T}$ & coding & 0.36 & 0.46 & $0.45(0.28-0.71)$ & $6.34 \times 10^{-04}$ & $6.9 \times 10^{-4}$ \\
\hline rs877111 & $6 \mathrm{p} 21.1$ & 6 & $\mathrm{C}$ & intergenic & 0.4 & 0.23 & $2.40(1.45-3.97)$ & $6.36 \times 10^{-04}$ & $5.9 \times 10^{-4}$ \\
\hline rs753712 & $10 q 26.3$ & 10 & $\mathrm{~T}$ & intergenic & 0.38 & 0.57 & $0.48(0.31-0.73)$ & $6.39 \times 10^{-04}$ & $5.9 \times 10^{-4}$ \\
\hline rs1430838 & $8 q 13.3$ & 8 & $\mathrm{C}$ & intergenic & 0.52 & 0.35 & $2.28(1.42-3.67)$ & $6.48 \times 10^{-04}$ & $5.9 \times 10^{-4}$ \\
\hline rs2116078 & $8 q 13.3$ & 8 & G & intergenic & 0.52 & 0.35 & $2.28(1.42-3.67)$ & $6.48 \times 10^{-04}$ & $5.9 \times 10^{-4}$ \\
\hline rs2615874 & ATRNL1 & 10 & $\mathrm{G}$ & intron & 0.4 & 0.09 & $3.12(1.62-5.99)$ & $6.51 \times 10^{-04}$ & $5 \times 10^{-4}$ \\
\hline
\end{tabular}

${ }^{*} \mathrm{OR}_{\text {interaction }}$ per allele $\left(\mathrm{OR}_{\text {allele }}\right)$ for the additive model; ${ }^{* *} P$ value adjusted for genomic control; ${ }^{\dagger \dagger} \mathrm{P}$ value permutation $=$ point wise $\mathrm{P}$ value from maxT permutation analysis after 10,000 permutation

$\mathrm{Chr}=$ Chromosome, $\mathrm{SNP}=$ single nucleotide polymorphism 
Appendix Table 13: List of single nucleotide polymorphisms that showed age-specific associations based on the $P$ value and were statistically correlated

\begin{tabular}{|c|c|c|c|}
\hline Chr A & SNP 1 & Chr B & SNP 2 \\
\hline 2 & rs1521244 & 2 & rs6739633 \\
\hline 4 & rs6834625 & 4 & rs1510136 \\
\hline 7 & rs10250709 & 7 & rs6972158 \\
\hline 8 & rs1430838 & 8 & rs2116078 \\
\hline 13 & rs6492498 & 13 & rs2065212 \\
\hline 14 & rs7157104 & 14 & rs2274068 \\
\hline 14 & rs7157104 & 14 & rs8021355 \\
\hline 14 & rs7157104 & 14 & rs7144565 \\
\hline 14 & rs2274068 & 14 & rs8021355 \\
\hline 14 & rs2274068 & 14 & rs7144565 \\
\hline 14 & rs8021355 & 14 & rs7144565 \\
\hline 14 & rs3825594 & 14 & rs2101919 \\
\hline 16 & rs1151277 & 16 & rs2388773 \\
\hline 19 & rs4254428 & 19 & rs6510588 \\
\hline 21 & rs16979806 & 21 & rs377621 \\
\hline 22 & rs2899293 & 22 & rs6519091 \\
\hline 22 & rs6519091 & 22 & rs139886 \\
\hline 22 & rs139866 & 22 & rs139886 \\
\hline
\end{tabular}

$\mathrm{SNP}=$ single nucleotide polymorphism

Note: Chromosome A and Chromosome B are same chromosome.

SNP1 and SNP2 and statistically correlated SNPs 
Appendix Table 14: Top one hundred SNPs associated with childhood ALL risk with age-specificity, results based on the highest effect sizes $\quad(\mathrm{P}<0.05)$

\begin{tabular}{|c|c|c|c|c|c|c|c|c|c|}
\hline \multirow[b]{2}{*}{ SNP } & \multirow[b]{2}{*}{ Gene/region } & \multirow[b]{2}{*}{ Chr } & \multirow[b]{2}{*}{$\begin{array}{l}\text { Minor } \\
\text { allele }\end{array}$} & \multirow[b]{2}{*}{ Feature } & \multicolumn{2}{|c|}{$\begin{array}{l}\text { Minor allele } \\
\text { frequency }\end{array}$} & \multirow[b]{2}{*}{ ORallele (95\% CI)* } & \multirow[b]{2}{*}{$P$ value $* *$} & \multirow[b]{2}{*}{$\begin{array}{c}\text { P value } \\
\text { (permutation) }\end{array}$} \\
\hline & & & & & $1-5$ year & 6-10 year & & & \\
\hline rs17109582 & DIO1 & 1 & $\mathrm{C}$ & intron & 0.09 & 0.007 & $12.46(1.67-93.01)$ & 0.01 & 0.006 \\
\hline rs7304215 & TMEM132D & 12 & $\mathrm{G}$ & intron & 0.07 & 0.007 & $11.25(1.47-85.90)$ & 0.02 & 0.01 \\
\hline rs10999147 & AIFM2 & 10 & $\mathrm{G}$ & coding & 0.07 & 0.007 & $10.89(1.43-83.02)$ & 0.02 & 0.01 \\
\hline rs7330286 & $13 q 33.2$ & 13 & $\mathrm{~T}$ & intergenic & 0.07 & 0.007 & $10.01(1.33-75.63)$ & 0.03 & 0.01 \\
\hline rs41309927 & SEC23B & 20 & $\mathrm{~A}$ & coding & 0.07 & 0.007 & $9.70(1.28-73.39)$ & 0.03 & 0.02 \\
\hline rs36086854 & CFAP99 & 4 & $\mathrm{~A}$ & coding & 0.06 & 0.007 & $9.42(1.23-72.26)$ & 0.03 & 0.02 \\
\hline rs17409162 & ANO10 & 3 & $\mathrm{~A}$ & coding & 0.06 & 0.007 & $9.24(1.20-71.06)$ & 0.03 & 0.02 \\
\hline rs13129052 & $4 \mathrm{p} 16.3$ & 4 & $\mathrm{G}$ & intron & 0.06 & 0.007 & $9.24(1.20-71.06)$ & 0.03 & 0.02 \\
\hline rs9856456 & $3 \mathrm{p} 22.1$ & 3 & $\mathrm{C}$ & intergenic & 0.06 & 0.007 & $8.78(1.14-67.62)$ & 0.04 & 0.02 \\
\hline rs6927353 & $6 q 26$ & 6 & A & intergenic & 0.06 & 0.007 & $8.68(1.16-64.90)$ & 0.04 & 0.02 \\
\hline rs11651675 & TMC8 & 17 & $\mathrm{~A}$ & coding & 0.05 & 0.007 & $8.65(1.11-67.50)$ & 0.04 & 0.03 \\
\hline rs10109794 & TRIM55 & 8 & $\mathrm{~T}$ & intron & 0.06 & 0.007 & $8.58(1.13-65.16)$ & 0.04 & 0.02 \\
\hline rs10099957 & TRIM55 & 8 & $\mathrm{~A}$ & intron & 0.11 & 0.007 & $8.58(2.00-36.93)$ & 0.004 & 0.001 \\
\hline rs56291145 & ATP12A & 13 & $\mathrm{C}$ & coding & 0.06 & 0.007 & $8.40(1.09-64.78)$ & 0.04 & 0.02 \\
\hline rs2909644 & $18 \mathrm{q} 22$ & 18 & $\mathrm{G}$ & intergenic & 0.06 & 0.007 & $8.40(1.09-64.78)$ & 0.04 & 0.03 \\
\hline rs6093579 & PTPRT & 20 & $\mathrm{~T}$ & intron & 0.06 & 0.007 & $8.40(1.09-64.78)$ & 0.04 & 0.02 \\
\hline rs17039265 & $1 \mathrm{p} 36.21$ & 1 & $\mathrm{~A}$ & intergenic & 0.06 & 0.007 & $8.29(1.08-63.96)$ & 0.04 & 0.02 \\
\hline rs9728577 & PRAMEF2 & 1 & $\mathrm{~A}$ & coding & 0.06 & 0.007 & $8.29(1.08-63.96)$ & 0.04 & 0.02 \\
\hline rs75411676 & PRAMEF2 & 1 & $\mathrm{~T}$ & coding & 0.06 & 0.007 & $8.29(1.08-63.96)$ & 0.04 & 0.02 \\
\hline rs36068434 & CDS1 & 4 & $\mathrm{~T}$ & coding & 0.06 & 0.007 & $8.29(1.08-63.96)$ & 0.04 & 0.02 \\
\hline
\end{tabular}


Appendix Table 14 continued....

\begin{tabular}{|c|c|c|c|c|c|c|c|c|c|}
\hline & & & & & $\begin{array}{l}\text { Minc } \\
\text { frec }\end{array}$ & $\begin{array}{l}\text { allele } \\
\text { ency }\end{array}$ & & & \\
\hline SNP & Gene/region & Chr & $\begin{array}{l}\text { Minor } \\
\text { allele }\end{array}$ & Feature & 1-5 year & 6-10 year & ORallele (95\% CI)* & $P$ value** & $\begin{array}{c}\text { P value } \\
\text { (permutation) }\end{array}$ \\
\hline rs11916525 & CEP70 & 3 & $\mathrm{C}$ & coding & 0.06 & 0.007 & $8.29(1.07-64.03)$ & 0.04 & 0.02 \\
\hline rs3204798 & PRAMEF2 & 1 & G & intron & 0.06 & 0.007 & $8.25(1.07-63.64)$ & 0.04 & 0.02 \\
\hline rs35340237 & CEP70 & 3 & $\mathrm{C}$ & coding & 0.06 & 0.007 & $8.21(1.06-63.41)$ & 0.04 & 0.02 \\
\hline rs8176018 & KEL & 7 & $\mathrm{~T}$ & intron & 0.06 & 0.007 & $8.19(1.07-62.68)$ & 0.04 & 0.02 \\
\hline rs1835154 & $2 \mathrm{q} 24.1$ & 2 & G & intergenic & 0.06 & 0.007 & $8.05(1.06-61.21)$ & 0.04 & 0.03 \\
\hline rs78323957 & SLC10A5 & 8 & $\mathrm{G}$ & coding & 0.05 & 0.007 & $8.02(1.03-62.12)$ & 0.05 & 0.03 \\
\hline rs16909509 & ZFAND1 & 8 & $\mathrm{~T}$ & intron & 0.05 & 0.007 & $8.02(1.03-62.12)$ & 0.05 & 0.03 \\
\hline rs3729598 & LIFR & 5 & $\mathrm{G}$ & intron & 0.05 & 0.007 & $7.94(1.03-61.48)$ & 0.05 & 0.04 \\
\hline rs568760 & HCN4 & 15 & $\mathrm{~T}$ & intron & 0.06 & 0.007 & $7.78(1.02-59.39)$ & 0.05 & 0.03 \\
\hline rs1800141 & $L R P 1$ & 12 & A & coding & 0.05 & 0.007 & $7.75(1.00-59.98)$ & 0.05 & 0.04 \\
\hline rs35384751 & $F Y B$ & 5 & $\mathrm{~T}$ & coding & 0.06 & 0.007 & $7.68(1.01-58.62)$ & 0.05 & 0.03 \\
\hline rs11175471 & RASSF3 & 12 & $\mathrm{C}$ & intron & 0.1 & 0.01 & $7.56(1.77-32.29)$ & 0.006 & 0.004 \\
\hline rs2389891 & $4 q 26$ & 4 & $\mathrm{G}$ & intergenic & 0.09 & 0.01 & $7.21(1.66-31.40)$ & 0.008 & 0.005 \\
\hline rs35424709 & ATP13A4 & 3 & A & coding & 0.11 & 0.02 & $6.74(1.95-23.25)$ & 0.003 & 0.009 \\
\hline rs12188300 & $5 q 33.3$ & 5 & $\mathrm{~T}$ & intergenic & 0.08 & 0.01 & $6.58(1.51-28.74)$ & 0.01 & 0.007 \\
\hline rs7303641 & RASSF3 & 12 & $\mathrm{G}$ & intron & 0.09 & 0.01 & $6.57(1.54-28.09)$ & 0.01 & 0.004 \\
\hline rs34139105 & PRRG4 & 11 & $\mathrm{~A}$ & coding & 0.08 & 0.01 & $6.36(1.44-28.06)$ & 0.01 & 0.009 \\
\hline rs10500248 & $19 q 12$ & 19 & $\mathrm{C}$ & intergenic & 0.08 & 0.01 & $6.34(1.45-27.77)$ & 0.01 & 0.01 \\
\hline rs2124906 & $19 q 12$ & 19 & $\mathrm{G}$ & intergenic & 0.08 & 0.01 & $6.34(1.45-27.77)$ & 0.01 & 0.01 \\
\hline rs7492685 & $14 \mathrm{q} 21.3$ & 14 & $\mathrm{C}$ & intergenic & 0.07 & 0.01 & $6.26(1.38-28.46)$ & 0.02 & 0.009 \\
\hline rs276725 & $19 q 12$ & 19 & $\mathrm{C}$ & intergenic & 0.08 & 0.01 & $6.05(1.38-26.56)$ & 0.02 & 0.007 \\
\hline
\end{tabular}


Appendix Table 14 continued....

\begin{tabular}{|c|c|c|c|c|c|c|c|c|c|}
\hline \multirow[b]{2}{*}{ SNP } & \multirow[b]{2}{*}{ Gene/region } & \multirow[b]{2}{*}{ Chr } & \multirow[b]{2}{*}{$\begin{array}{l}\text { Minor } \\
\text { allele }\end{array}$} & \multirow[b]{2}{*}{ Feature } & \multicolumn{2}{|c|}{$\begin{array}{l}\text { Minor allele } \\
\text { frequency }\end{array}$} & \multirow[b]{2}{*}{ ORallele $(95 \% \text { CI })^{*}$} & \multirow[b]{2}{*}{$P$ value** } & \multirow[b]{2}{*}{$\begin{array}{c}\text { P value } \\
\text { (permutation) }\end{array}$} \\
\hline & & & & & 1-5 year & 6-10 year & & & \\
\hline rs6851586 & $4 p 15.2$ & 4 & $\mathrm{~A}$ & intergenic & 0.09 & 0.01 & $5.87(1.38-24.92)$ & 0.02 & 0.01 \\
\hline rs16871434 & KCNIP4 & 4 & $\mathrm{~A}$ & intron & 0.08 & 0.01 & $5.79(1.34-25.04)$ & 0.02 & 0.01 \\
\hline rs28421666 & $6 \mathrm{p} 21.3$ & 6 & $\mathrm{G}$ & intergenic & 0.1 & 0.02 & $5.58(1.63-19.06)$ & 0.006 & 0.003 \\
\hline rs17055478 & $13 \mathrm{q} 21.1$ & 13 & $\mathrm{~A}$ & intergenic & 0.07 & 0.01 & $5.58(1.26-24.70)$ & 0.02 & 0.02 \\
\hline rs1582274 & $7 q 31.1$ & 7 & A & intergenic & 0.07 & 0.01 & $5.48(1.24-24.24)$ & 0.02 & 0.02 \\
\hline rs6572051 & LOC644919 & 14 & $\mathrm{G}$ & intron & 0.07 & 0.01 & $5.40(1.22-23.85)$ & 0.03 & 0.02 \\
\hline rs9407838 & SH3GL2 & 9 & $\mathrm{~T}$ & intron & 0.11 & 0.02 & $5.32(1.58-17.89)$ & 0.007 & 0.004 \\
\hline rs72838683 & PDZD7 & 10 & $\mathrm{~T}$ & 3'UTR & 0.07 & 0.01 & $5.31(1.22-23.05)$ & 0.03 & 0.02 \\
\hline rs11250832 & $10 \mathrm{p} 15.3$ & 10 & $\mathrm{~T}$ & intergenic & 0.1 & 0.02 & $5.29(1.56-17.97)$ & 0.008 & 0.005 \\
\hline rs16827743 & $3 q 13.32$ & 3 & $\mathrm{~T}$ & intergenic & 0.07 & 0.01 & $5.28(1.20-23.32)$ & 0.03 & 0.03 \\
\hline rs12705300 & $7 q 22.3$ & 7 & $\mathrm{~A}$ & intron & 0.12 & 0.02 & $5.27(1.61-17.30)$ & 0.006 & 0.003 \\
\hline rs1915164 & $15 q 21.1$ & 15 & $\mathrm{~T}$ & intergenic & 0.07 & 0.01 & $5.24(1.20-22.95)$ & 0.03 & 0.01 \\
\hline rs12083795 & $1 \mathrm{p} 34.3$ & 1 & $\mathrm{~T}$ & intergenic & 0.07 & 0.01 & $5.21(1.18-23.08)$ & 0.03 & 0.02 \\
\hline rs9861022 & $3 q 13.32$ & 3 & $\mathrm{C}$ & intergenic & 0.07 & 0.01 & $5.14(1.18-22.38)$ & 0.03 & 0.02 \\
\hline rs16964079 & $\begin{array}{c}\text { C15orf41/ } \\
\text { CSNK1A1P1 }\end{array}$ & 15 & A & 3'UTR & 0.07 & 0.01 & $5.10(1.17-22.22)$ & 0.03 & 0.02 \\
\hline rs10157510 & $1 \mathrm{q} 24.3$ & 1 & $\mathrm{~T}$ & intergenic & 0.08 & 0.01 & $5.00(1.18-21.27)$ & 0.03 & 0.02 \\
\hline rs10224856 & VPS41 & 7 & $\mathrm{~A}$ & intron & 0.07 & 0.01 & $4.95(1.12-21.93)$ & 0.04 & 0.03 \\
\hline rs1728850 & $15 q 21.3$ & 15 & $\mathrm{G}$ & intergenic & 0.07 & 0.01 & $4.95(1.12-21.93)$ & 0.04 & 0.03 \\
\hline rs17055548 & $13 q 21.1$ & 13 & $\mathrm{G}$ & intergenic & 0.06 & 0.01 & $4.94(1.11-22.03)$ & 0.04 & 0.03 \\
\hline rs10133677 & MDGA2 & 14 & $\mathrm{G}$ & intron & 0.16 & 0.04 & $4.92(1.98-12.23)$ & 0.0006 & 0.0004 \\
\hline rs3130161 & $6 \mathrm{p} 21.3$ & 6 & $\mathrm{C}$ & intergenic & 0.07 & 0.01 & $4.89(1.13-21.23)$ & 0.03 & 0.04 \\
\hline
\end{tabular}


Appendix Table 14 continued....

\begin{tabular}{|c|c|c|c|c|c|c|c|c|c|}
\hline & & & & & $\begin{array}{l}\text { Minc } \\
\text { frec }\end{array}$ & $\begin{array}{l}\text { allele } \\
\text { ency }\end{array}$ & & & \\
\hline SNP & Gene/region & Chr & $\begin{array}{l}\text { Minor } \\
\text { allele }\end{array}$ & Feature & 1-5 year & 6-10 year & ORallele $(95 \% \text { CI })^{*}$ & $P$ value** & $\begin{array}{c}\text { P value } \\
\text { (permutation) }\end{array}$ \\
\hline rs1000521 & SLC8A3 & 14 & $\mathrm{G}$ & intron & 0.11 & 0.02 & $4.87(1.46-16.18)$ & 0.01 & 0.005 \\
\hline rs12257453 & ARMC3 & 10 & G & intron & 0.07 & 0.01 & $4.86(1.12-21.19)$ & 0.04 & 0.03 \\
\hline rs11833839 & EP400 & 12 & $\mathrm{~T}$ & coding & 0.06 & 0.01 & $4.85(1.09-21.59)$ & 0.04 & 0.03 \\
\hline rs3790126 & EMP2 & 16 & A & 3'UTR & 0.08 & 0.01 & $4.85(1.15-20.38)$ & 0.03 & 0.02 \\
\hline rs16950438 & $16 q 12.1$ & 16 & $\mathrm{C}$ & intergenic & 0.1 & 0.02 & $4.84(1.44-16.25)$ & 0.01 & 0.005 \\
\hline rs6151599 & $\begin{array}{l}\text { MSH3/ } \\
\text { DHFR }\end{array}$ & 5 & G & intron & 0.1 & 0.02 & $4.82(1.45-16.10)$ & 0.01 & 0.008 \\
\hline rs4546429 & $5 \mathrm{p} 13.1$ & 5 & $\mathrm{~T}$ & intergenic & 0.07 & 0.01 & $4.79(1.10-20.82)$ & 0.04 & 0.03 \\
\hline rs11234604 & C11orf73 & 11 & A & 5'UTR & 0.06 & 0.01 & $4.78(1.07-21.24)$ & 0.04 & 0.03 \\
\hline rs6708453 & $2 q 21.1$ & 2 & $\mathrm{C}$ & intergenic & 0.17 & 0.04 & $4.72(1.92-11.62)$ & 0.0007 & 0.0007 \\
\hline rs3805114 & ABCC5 & 3 & $\mathrm{C}$ & 3'UTR & 0.06 & 0.01 & $4.71(1.06-20.95)$ & 0.04 & 0.04 \\
\hline rs9328207 & $6 \mathrm{p} 25.2$ & 6 & $\mathrm{~T}$ & intergenic & 0.06 & 0.01 & $4.71(1.06-20.95)$ & 0.04 & 0.04 \\
\hline rs2864680 & FSTL4 & 5 & A & intron & 0.07 & 0.01 & $4.71(1.10-20.10)$ & 0.04 & 0.02 \\
\hline rs4741086 & $9 \mathrm{p} 24.3$ & 9 & $\mathrm{~A}$ & intergenic & 0.17 & 0.04 & $4.67(1.91-11.42)$ & 0.0007 & 0.0009 \\
\hline rs10514074 & $18 \mathrm{q} 22.3$ & 18 & $\mathrm{C}$ & intergenic & 0.1 & 0.02 & $4.66(1.37-15.88)$ & 0.01 & 0.01 \\
\hline rs41285557 & MAML1 & 5 & A & coding & 0.06 & 0.01 & $4.65(1.02-21.12)$ & 0.05 & 0.03 \\
\hline rs7620914 & $3 p 22.3$ & 3 & A & intron & 0.14 & 0.04 & $4.64(1.75-12.32)$ & 0.002 & 0.001 \\
\hline rs7091901 & $10 p 11.22$ & 10 & $\mathrm{~T}$ & intergenic & 0.09 & 0.02 & $4.64(1.35-15.96)$ & 0.02 & 0.01 \\
\hline rs8100295 & $19 q 13.42$ & 19 & $\mathrm{~T}$ & intergenic & 0.06 & 0.01 & $4.62(1.04-20.60)$ & 0.04 & 0.04 \\
\hline rs17407084 & $A K 8$ & 9 & $\mathrm{C}$ & coding & 0.06 & 0.01 & $4.58(1.03-20.51)$ & 0.05 & 0.03 \\
\hline rs222314 & $7 \mathrm{p} 21.3$ & 7 & G & intergenic & 0.2 & 0.05 & $4.52(1.98-10.35)$ & 0.0003 & 0.0002 \\
\hline rs7770942 & $6 \mathrm{p} 25.3$ & 6 & $\mathrm{~T}$ & intergenic & 0.09 & 0.02 & $4.51(1.31-15.54)$ & 0.05 & 0.01 \\
\hline
\end{tabular}


Appendix Table 14 continued....

\begin{tabular}{|c|c|c|c|c|c|c|c|c|c|}
\hline \multirow[b]{2}{*}{ SNP } & \multirow[b]{2}{*}{ Gene/region } & \multirow[b]{2}{*}{ Chr } & \multirow[b]{2}{*}{$\begin{array}{l}\text { Minor } \\
\text { allele }\end{array}$} & \multirow[b]{2}{*}{ Feature } & \multicolumn{2}{|c|}{$\begin{array}{c}\text { Minor allele } \\
\text { frequency }\end{array}$} & \multirow[b]{2}{*}{ ORallele $(95 \% \text { CI })^{*}$} & \multirow[b]{2}{*}{$P$ value** } & \multirow[b]{2}{*}{$\begin{array}{c}\text { P value } \\
\text { (permutation) }\end{array}$} \\
\hline & & & & & 1-5 year & 6-10 year & & & \\
\hline rs16916188 & $R B M 12 B$ & 8 & $\mathrm{G}$ & coding & 0.06 & 0.01 & $4.51(1.01-20.12)$ & 0.05 & 0.03 \\
\hline rs17140942 & $R B F O X 1$ & 16 & $\mathrm{G}$ & intron & 0.06 & 0.01 & $4.51(1.01-20.12)$ & 0.05 & 0.03 \\
\hline rs7172316 & $15 q 24.1$ & 15 & $\mathrm{G}$ & intergenic & 0.07 & 0.02 & $4.49(1.03-19.51)$ & 0.05 & 0.03 \\
\hline rs1209145 & $14 q 22.3$ & 14 & $\mathrm{G}$ & intergenic & 0.17 & 0.05 & $4.48(1.91-10.50)$ & 0.0006 & 0.0003 \\
\hline rs10902652 & $1 \mathrm{p} 36.11$ & 1 & $\mathrm{C}$ & intergenic & 0.06 & 0.01 & $4.46(1.02-19.61)$ & 0.05 & 0.04 \\
\hline rs1457759 & $5 q 22.3$ & 5 & $\mathrm{C}$ & intergenic & 0.09 & 0.02 & $4.44(1.31-15.08)$ & 0.02 & 0.01 \\
\hline rs10826912 & $10 p 11.22$ & 10 & $\mathrm{G}$ & intergenic & 0.08 & 0.02 & $4.44(1.29-15.33)$ & 0.02 & 0.008 \\
\hline rs61744122 & VWA3A & 16 & A & intron & 0.06 & 0.01 & $4.44(1.02-19.36)$ & 0.05 & 0.04 \\
\hline rs4748309 & RSU1 & 10 & $\mathrm{~A}$ & intron & 0.1 & 0.02 & $4.39(1.33-14.52)$ & 0.02 & 0.008 \\
\hline rs8013602 & $14 q 21.2$ & 14 & A & intergenic & 0.06 & 0.01 & $4.39(1.00-19.23)$ & 0.05 & 0.04 \\
\hline rs9560160 & $13 q 34$ & 13 & $\mathrm{C}$ & intergenic & 0.13 & 0.03 & $4.38(1.54-12.49)$ & 0.005 & 0.003 \\
\hline rs1755588 & ACOT11 & 1 & $\mathrm{~T}$ & intron & 0.09 & 0.02 & $4.36(1.28-14.88)$ & 0.02 & 0.01 \\
\hline rs10493958 & $1 \mathrm{p} 21.2$ & 1 & A & intergenic & 0.07 & 0.01 & $4.32(1.01-18.51)$ & 0.05 & 0.03 \\
\hline rs7169431 & $15 q 21.3$ & 15 & $\mathrm{~A}$ & intergenic & 0.08 & 0.02 & $4.31(.124-14.96)$ & 0.02 & 0.02 \\
\hline rs17170286 & BBS9 & 7 & $\mathrm{C}$ & intron & 0.09 & 0.02 & $4.30(1.26-14.68)$ & 0.02 & 0.02 \\
\hline rs11945730 & $4 p 15.32$ & 4 & $\mathrm{G}$ & intergenic & 0.12 & 0.03 & $4.28(1.47-12.40)$ & 0.007 & 0.006 \\
\hline rs17149645 & $11 \mathrm{q} 14.2$ & 11 & $\mathrm{~T}$ & intergenic & 0.11 & 0.03 & $4.25(1.45-12.46)$ & 0.008 & 0.006 \\
\hline
\end{tabular}

$* \mathrm{OR}_{\text {interaction }}$ per allele $\left(\mathrm{OR}_{\text {allele }}\right)$ for the additive model; **P value adjusted for genomic control; ${ }^{\dagger} \mathrm{P}$ value permutation $=$ point wise $\mathrm{P}$ value from maxT permutation analysis after 10,000 permutation

$\mathrm{Chr}=$ Chromosome, $\mathrm{SNP}=$ single nucleotide polymorphism 
Appendix Table 15: List of single nucleotide polymorphisms that showed agespecific associations based on effect size and were statistically correlated

\begin{tabular}{|c|c|c|c|}
\hline Chr A & SNP 1 & Chr B & SNP 2 \\
\hline 1 & rs17039265 & 1 & rs9728577 \\
\hline 1 & rs17039265 & 1 & rs3204798 \\
\hline 1 & rs17039265 & 1 & rs75411676 \\
\hline 1 & rs9728577 & 1 & rs3204798 \\
\hline 1 & rs9728577 & 1 & rs75411676 \\
\hline 1 & rs3204798 & 1 & rs75411676 \\
\hline 3 & rs9861022 & 3 & rs16827743 \\
\hline 8 & rs78323957 & 8 & rs16909509 \\
\hline 10 & rs7091901 & 10 & rs10826912 \\
\hline 12 & rs11175471 & 12 & rs7303641 \\
\hline 13 & rs17055478 & 13 & rs17055548 \\
\hline 14 & rs4502127 & 14 & rs11157201 \\
\hline 19 & rs10500248 & 19 & rs2124906 \\
\hline
\end{tabular}

$\mathrm{SNP}=$ single nucleotide polymorphism

Note: Chromosome A and Chromosome B are same chromosome. SNP1 and SNP2 and statistically correlated SNPs 
Appendix Table 16: Bioinformatic analysis of genetic risk markers for the top one hundred age-specific associations with childhood ALL with the lowest $P$ values

\begin{tabular}{|c|c|c|c|c|c|c|c|}
\hline SNP & Chr & $\begin{array}{c}\text { RegulomeDB } \\
\text { score }^{\dagger}\end{array}$ & F-SNP ${ }^{* *}$ & Gene & $\begin{array}{c}\text { Correlated } \\
\text { SNP }\end{array}$ & $\underset{\text { score }^{\dagger}}{\text { RegulomeDB }}$ & $\begin{array}{c}\text { Gene } \\
\text { (correlated SNP) }\end{array}$ \\
\hline rs10505918 & 12 & $\mathrm{NS}$ & NS & SOX5 & $\mathrm{Cl}_{2}$ & 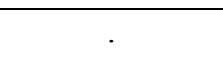 & . \\
\hline rs11997355 & 8 & $3 \mathbf{a}$ & NS & HNF4G-ZFHX4 & . & . & . \\
\hline rs2388773 & 16 & 5 & NS & $I R X-C R N D E 3$ & . & • & . \\
\hline rs665159 & 1 & 5 & 0.5 & CHRM3 & rs112493720 & $2 b$ & CHRM3 \\
\hline rs3954950 & 9 & 5 & NS & RFX-GLIS33 & . & . & . \\
\hline rs7818844 & 8 & 5 & NS & CYCSP3-RPL10AP3 & . & . & . \\
\hline rs6972158 ${ }^{*+\dagger}$ & 7 & 5 & 0.41 & NPSR1 & . & . & . \\
\hline rs7320982 & 13 & 5 & NS & MIRHG1-GPC5 & . & . & . \\
\hline rs7583258 & 2 & 5 & 0.1 & TEX41-ACVR2A & . & . & . \\
\hline rs1882591 & 16 & NS & NS & IRX-CRNDE3 & rs1151271 & $3 a$ & IRX3-CRNDE \\
\hline rs7318021 & 13 & NS & NS & KRT18P27-RNU6-75P & rs7996128 & $3 a$ & KRT18P27-RNU6-75P \\
\hline rs7016929 & 8 & 5 & NS & $\begin{array}{c}\text { KHDRBS3- } \\
\text { LOC101927892 }\end{array}$ & . & . & . \\
\hline rs10079494 & 5 & 4 & NS & MSH3-RASGRF2 & rs7708505 & $2 b$ & MSH3-RASGRF2 \\
\hline rs10250709 & 7 & 5 & 0.18 & NPSR1 & . & . & . \\
\hline rs7720650 & 5 & 5 & NS & WNT8A-NME5 & rs56331448 & $2 b$ & WNT8A-NME5 \\
\hline rs2949827 & 7 & NS & NS & GPR85-PPP1R3A & . & . & . \\
\hline rs2396653 & 7 & 6 & NS & GPR85-PPP1R3A & . & . & . \\
\hline rs202050948 & 19 & 5 & NS & CD97 & . & . & . \\
\hline rs1191818 & 2 & $2 \mathbf{a}$ & 0.1 & MATN3-LAPTM4A & . & . & . \\
\hline rs3800292 & 6 & 5 & 0.21 & GNMT & rs3831135 & $2 b$ & PEX6 \\
\hline rs3800292 & 6 & 5 & 0.21 & GNMT & rs2274518 & $2 b$ & PEX6 \\
\hline
\end{tabular}


Appendix Table 16 continued....

\begin{tabular}{|c|c|c|c|c|c|c|c|}
\hline SNP & Chr & $\begin{array}{c}\text { RegulomeDB } \\
\text { score }^{\dagger}\end{array}$ & F-SNP ${ }^{* *}$ & Gene & $\begin{array}{l}\text { Correlated } \\
\text { SNP }\end{array}$ & $\begin{array}{c}\text { RegulomeDB } \\
\text { score }^{\dagger}\end{array}$ & $\begin{array}{c}\text { Gene } \\
\text { (correlated SNP) }\end{array}$ \\
\hline rs3800292 & 6 & 5 & 0.21 & GNMT & rs 8540 & $2 \mathrm{~b}$ & KLHDC3 \\
\hline rs1881116 & 7 & 5 & NS & C7orf50 & . & . & . \\
\hline rs4821717 & 22 & 5 & 0.5 & GCAT-GALR3 & rs139852 & 1b & EIF3EIP \\
\hline rs4821717 & 22 & 5 & 0.5 & GCAT-GALR3 & rs7290156 & 1f & GALR3 \\
\hline rs2212824 & 21 & 6 & NS & ADAMTS5-GPXP2 & . & . & . \\
\hline rs7157104 & 14 & 6 & NS & KRT18P6-INSM2 & . & . & . \\
\hline rs2051713 & 12 & 6 & NS & MRPL2P1-C12orf37 & . & . & . \\
\hline rs2125649 & 8 & NS & NS & RP11-24P4.1-GFRA2 & . & . & . \\
\hline rs6739633 & 2 & 6 & 0.1 & TRIB2-FAM84A & . & . & . \\
\hline rs738027 & 12 & 6 & NS & PYROXD1 & rs7133853 & $3 a$ & PYROXD1 \\
\hline rs237320 & 17 & 5 & NS & MAP2K4-MYOCD & rs $237318^{\dagger \dagger}$ & $2 b$ & MAP2K4-MYOCD \\
\hline rs139886 & 22 & 1f & 0.18 & SOX10 & rs139892 & 1f & POLR2F \\
\hline rs13116233 & 4 & 6 & 0.1 & AGA-LINC01098 & . & . & . \\
\hline rs6519091 & 22 & $2 b$ & NS & MICALL1 & rs4821723 & 1f & MICALL1 \\
\hline rs6109487 & 20 & 5 & NS & PA2G4P2-SPTLC3 & rs73088401 & $2 \mathrm{c}$ & PA2G4P2-SPTLC3 \\
\hline rs1151277 & 16 & 6 & NS & IRX-CRNDE3 & . & . & . \\
\hline rs6052937 & 20 & 5 & NS & SLC23A2 & . & . & . \\
\hline rs34099167* & 4 & NS & 0 & NEK1 & rs6818590 & $2 b$ & NEK1 \\
\hline rs7722067 & 5 & NS & 0.1 & ATP6AP1L-RPL5P16 & . & . & . \\
\hline rs1004327 & 4 & $\mathbf{3 a}$ & 0.24 & CLNK & . & . & . \\
\hline rs3993401 & 12 & NS & NS & C12orf26- TMTC2 & . & . & . \\
\hline rs6492498 & 13 & 4 & NS & KRT18P27-LINC01049 & . & . & . \\
\hline rs2806907 & 13 & 6 & NS & SIAHЗ-ZCЗH13 & rs9522975 & $3 a$ & KRT18P27 - LINC01049 \\
\hline
\end{tabular}


Appendix Table 16 continued....

\begin{tabular}{|c|c|c|c|c|c|c|c|}
\hline SNP & Chr & $\begin{array}{c}\text { RegulomeDB } \\
\text { score }^{\dagger} \\
\end{array}$ & F-SNP ${ }^{* *}$ & Gene & $\begin{array}{c}\text { Correlated } \\
\text { SNP } \\
\end{array}$ & $\begin{array}{c}\text { RegulomeDB } \\
\text { score }^{\dagger} \\
\end{array}$ & $\begin{array}{c}\text { Gene } \\
(\text { correlated SNP) }\end{array}$ \\
\hline rs2101919 & 14 & 6 & 0.37 & NID2 & 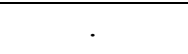 & (1) & . \\
\hline rs2899293 & 22 & 6 & 0.5 & ANKRD54 & . & . & . \\
\hline rs1028695 & 13 & NS & 0.5 & SPATA13 & rs4821722 & $1 \mathrm{f}$ & EIF3L \\
\hline rs1028695 & 13 & NS & 0.5 & SPATA13 & rs 4821723 & $1 \mathrm{f}$ & MICALL1 \\
\hline rs2185230 & 9 & NS & 0.5 & PCSK5 & . & . & . \\
\hline rs4254428 & 19 & NS & NS & ZNF420 & rs2562599 & $1 \mathrm{f}$ & ZNF568-ZNF420 \\
\hline rs4254428 & 19 & NS & NS & ZNF420 & rs 1402468 & $1 \mathrm{f}$ & ZNF420 \\
\hline rs4254428 & 19 & NS & NS & ZNF420 & rs11666786 & $1 f$ & ZNF420-ZNF585A \\
\hline rs4254428 & 19 & NS & NS & ZNF420 & rs7245882 & $1 \mathrm{f}$ & ZNF420 \\
\hline rs139866 & 22 & NS & NS & POLR2F & rs139886 & $1 \mathrm{f}$ & SOX10 \\
\hline rs139866 & 22 & NS & NS & POLR2F & rs139892 & $1 \mathrm{f}$ & POLR2F \\
\hline rs5028798 & 11 & 5 & NS & ELF5- EHF & rs12793417 & $2 b$ & ELF5- EHF \\
\hline rs2065212 & 13 & NS & NS & KRT18P27-LINC01049 & rs9522975 & $3 a$ & KRT18P27-LINC01049 \\
\hline rs222314 & 7 & NS & NS & LOC340268-HSPA8P8 & . & . & . \\
\hline rs1894381 & 16 & NS & NS & TMC5 & . & . & . \\
\hline rs9809647 & 3 & 6 & 0.24 & PYDC2-FGF12 & . & . & . \\
\hline rs898982 & 1 & 5 & 0.1 & POU3F1- RRAGC & rs77420329 & $2 \mathrm{a}$ & POU3F1- RRAGC \\
\hline rs1868368 & 8 & 5 & NS & $\begin{array}{c}\text { KHDRBS3- } \\
\text { LOC101927892 }\end{array}$ & . & . & . \\
\hline rs9392857 & 6 & 5 & NS & EXOC2-OC101927691 & . & . & . \\
\hline rs8014810 & 14 & NS & NS & BRMS1L & rs7152711 & $3 a$ & GARNL1 \\
\hline rs8014810 & 14 & NS & NS & BRMS1L & rs10146494 & $3 a$ & BRMS1L \\
\hline rs8014810 & 14 & NS & NS & BRMS1L & rs71124724 & $3 a$ & BRMS1L-LOC644584 \\
\hline
\end{tabular}


Appendix Table 16 continued....

\begin{tabular}{|c|c|c|c|c|c|c|c|}
\hline SNP & Chr & $\begin{array}{c}\text { RegulomeDB } \\
\text { score }^{\dagger}\end{array}$ & F-SNP** & Gene & $\begin{array}{l}\text { Correlated } \\
\text { SNP }\end{array}$ & $\begin{array}{c}\text { RegulomeDB } \\
\text { score }^{\dagger}\end{array}$ & $\begin{array}{c}\text { Gene } \\
\text { (correlated SNP) }\end{array}$ \\
\hline rs8021355 & 14 & 6 & NS & RALGAPA1 & . & . & . \\
\hline rs7144565 & 14 & 6 & NS & RALGAPA1 & . & . & . \\
\hline rs2274068* & 14 & 4 & 0.65 & RALGAPA1 & . & . & . \\
\hline rs762562 & 19 & NS & 0.09 & ERCC1/CD3EAP & . & . & . \\
\hline rs4948088 & 7 & 5 & NS & GRB10-COBL & . & . & . \\
\hline rs3850942 & 11 & 4 & NS & $M A R K 2$ & rs61886104 & $2 b$ & MARK2 \\
\hline rs2899469 & 15 & 5 & NS & TNFAIP8L3-CYP19A1 & rs4551976 & $2 b$ & TNFAIP8L3-CYP19A1 \\
\hline rs8011343 & 14 & NS & 0.5 & MDGA2 & rs10133677 & $3 a$ & MDGA2 \\
\hline rs2031293 & 13 & 5 & NS & C13orf16-SOX1 & . & . & . \\
\hline rs11171543 & 12 & NS & NS & CPNE8- KIF21A & . & . & . \\
\hline rs11172024 & 12 & 6 & NS & KIF21A & rs11171955 & $3 a$ & KIF21A \\
\hline rs11172024 & 12 & 6 & NS & KIF21A & rs11171957 & $3 a$ & KIF21A \\
\hline rs11172024 & 12 & 6 & NS & KIF21A & rs12228664 & $3 a$ & KIF21A-BCD2 \\
\hline rs1510136 & 4 & 5 & NS & INPP4B-USP38 & rs11306676 & $2 b$ & INPP4B-USP38 \\
\hline rs16979806 & 21 & 4 & NS & $\begin{array}{c}\text { ADAMTS5- } \\
\text { LOC102724355 }\end{array}$ & . & . & . \\
\hline rs17017488 & 4 & 5 & 0.5 & CCSER1 & . & . & . \\
\hline rs10511980 & 9 & NS & NS & MAMDC2 & rs2209779 & $2 b$ & MAMDC2 \\
\hline rs2620870 & 12 & NS & NS & SLC16A7- USP15 & . & . & . \\
\hline rs377621 & 21 & NS & NS & $\begin{array}{c}\text { ADAMTS5- } \\
\text { LOC102724355 }\end{array}$ & rs368477 & $2 b$ & $\begin{array}{c}\text { ADAMTS5- } \\
\text { LOC102724355 }\end{array}$ \\
\hline rs6510588 & 19 & 6 & NS & ZNF420 & rs2562599 & 1f & ZNF568-ZNF420 \\
\hline rs6510588 & 19 & 6 & NS & ZNF420 & rs1402468 & $1 \mathrm{f}$ & ZNF420 \\
\hline rs6510588 & 19 & 6 & NS & ZNF420 & rs11666786 & 1f & ZNF420-ZNF585A \\
\hline
\end{tabular}


Appendix Table 16 continued....

\begin{tabular}{|c|c|c|c|c|c|c|c|}
\hline SNP & Chr & $\begin{array}{c}\text { RegulomeDB } \\
\text { score }^{\dagger}\end{array}$ & F-SNP ${ }^{* *}$ & Gene & $\begin{array}{l}\text { Correlated } \\
\text { SNP }\end{array}$ & $\begin{array}{c}\text { RegulomeDB } \\
\text { score }^{\dagger}\end{array}$ & $\begin{array}{c}\text { Gene } \\
\text { (correlated SNP) }\end{array}$ \\
\hline rs6510588 & 19 & 6 & NS & ZNF420 & rs 7245882 & $1 \mathrm{f}$ & ZNF420 \\
\hline rs10739501 & 9 & NS & NS & TLR4-BRINP1 & . & . & . \\
\hline rs6834625 & 4 & NS & 0.1 & INPP4B-USP38 & rs11306676 & $2 b$ & INPP4B-USP38 \\
\hline rs1209145 & 14 & 6 & NS & C14orf105-SLC35F4 & rs373690 & $1 \mathrm{f}$ & C14orf105-SLC35F4 \\
\hline rs1481046 & 8 & 6 & NS & NCOA2-TRAM1 & rs10096078 & $2 \mathrm{c}$ & NCOA2-TRAM1 \\
\hline rs924615 & 5 & NS & NS & FLJ41309-RPL5P16 & . & . & . \\
\hline rs26784 & 5 & 6 & 0.27 & MSH3 & . & . & . \\
\hline rs11120342 & 1 & NS & 0.24 & PTPN14 & . & . & . \\
\hline rs2830698 & 21 & NS & NS & $\begin{array}{c}\text { ADAMTS5- } \\
\text { LOC102724355 }\end{array}$ & rs 2830715 & $3 a$ & $\begin{array}{c}\text { ADAMTS5- } \\
\text { LOC102724355 }\end{array}$ \\
\hline rs6037033 & 20 & 5 & NS & VSX1- ENTPD6 & rs4815392 & $2 b$ & ENTPD6 \\
\hline rs12073211 & 1 & NS & 0.1 & MYSM1-JUN & rs11207297 & $2 b$ & MYSM1 \\
\hline rs3825594 & 14 & NS & NS & NID2 & . & . & . \\
\hline rs742390 & 1 & 4 & NS & RUNX3-SYF2 & . & . & . \\
\hline rs973358 & 17 & 5 & NS & SHISA6 & . & . & . \\
\hline rs10133677 & 14 & $3 \mathbf{a}$ & 0 & MDGA2 & . & . & . \\
\hline rs1493897 & 16 & 6 & NS & IRX3-CRNDE & . & . & . \\
\hline rs9694342 & 8 & $2 \mathbf{b}$ & 0 & AGO2 & . & . & . \\
\hline rs7952853 & 12 & 6 & 0.4 & OR6C2 & . & . & . \\
\hline rs6943934 & 7 & 5 & NS & C7orf50-ZFAND2A & rs73264941 & $2 b$ & C7orf50-ZFAND2A \\
\hline rs1610315 & 6 & 6 & NS & GNG5P1-PRR18 & . & . & . \\
\hline rs1521244 & 2 & 5 & NS & TRIB2-LOC100506474 & . & . & . \\
\hline rs9897794* & 17 & 1f & 0.37 & EFCAB5 & rs9890205 & 1d & EFCAB5 \\
\hline
\end{tabular}


Appendix Table 16 continued...

\begin{tabular}{|c|c|c|c|c|c|c|c|}
\hline SNP & Chr & $\begin{array}{c}\text { RegulomeDB } \\
\text { score }^{\dagger}\end{array}$ & F-SNP $^{* * *}$ & Gene & $\begin{array}{c}\text { Correlated } \\
\text { SNP }\end{array}$ & $\begin{array}{c}\text { RegulomeDB } \\
\text { score }^{\dagger}\end{array}$ & $\begin{array}{c}\text { Gene } \\
\text { (correlated SNP) }\end{array}$ \\
\hline rs877111 & 6 & 6 & 0.5 & C6orf223-MRPL14 & rs 7770652 & $1 \mathrm{f}$ & MRPL14 \\
\hline rs877111 & 6 & 6 & 0.5 & C6orf223-MRPL14 & rs4714739 & $1 \mathrm{f}$ & C6orf223-MRPL14 \\
\hline rs877111 & 6 & 6 & 0.5 & C6orf223-MRPL14 & rs4714741 & $1 \mathrm{f}$ & C6orf223-MRPL14 \\
\hline rs877111 & 6 & 6 & 0.5 & C6orf223-MRPL14 & rs1321097 & $1 \mathrm{f}$ & C6orf223-MRPL14 \\
\hline rs877111 & 6 & 6 & 0.5 & C6orf223-MRPL14 & rs910616 & $1 \mathrm{f}$ & C6orf223-MRPL14 \\
\hline rs877111 & 6 & 6 & 0.5 & C6orf223-MRPL14 & rs4714745 & 1f & C6orf223-MRPL14 \\
\hline rs877111 & 6 & 6 & 0.5 & C6orf223-MRPL14 & rs4714742 & $1 \mathrm{f}$ & C6orf223-MRPL14 \\
\hline rs877111 & 6 & 6 & 0.5 & C6orf223-MRPL14 & rs6883 & $1 \mathrm{f}$ & MRPL14 \\
\hline rs877111 & 6 & 6 & 0.5 & C6orf223-MRPL14 & rs4711767 & 1f & MRPL14 \\
\hline rs877111 & 6 & 6 & 0.5 & C6orf223-MRPL14 & rs910616 & $1 \mathrm{f}$ & C6orf223-MRPL14 \\
\hline rs877111 & 6 & 6 & 0.5 & C6orf223-MRPL14 & rs1935611 & 1f & MRPL14 \\
\hline rs877111 & 6 & 6 & 0.5 & C6orf223-MRPL14 & rs9462964 & $1 \mathrm{f}$ & TMEM63B \\
\hline rs877111 & 6 & 6 & 0.5 & C6orf223-MRPL14 & rs4714755 & 1f & TMEM63B \\
\hline rs753712 & 10 & 6 & NS & MKI67-MGMT & . & . & . \\
\hline rs1430838 & 8 & NS & NS & TRPA1-KCNB2 & . & . & . \\
\hline rs2116078 & 8 & 5 & NS & TRPA1-KCNB2 & . & . & . \\
\hline rs2615874 & 10 & 5 & NS & ATRNL1 & . & . & . \\
\hline
\end{tabular}

Note: Some associated SNPs showed multiple correlated functional variants

$\mathrm{Chr}=$ Chromosome; NS = No score; SNP = single nucleotide polymorphism; $*$ Missense; ${ }^{\dagger}$ SNPs change or create a binding site for transcription factors

${ }^{\dagger}$ RegulomDb predicts score from 1 to 7 . A score of one suggests SNPs have higher functionality and may play an important role in gene regulation. A score of 7 predicts least functionality

${ }^{* *}$ F-SNP predicts score from 0 to 1 . A score of one suggest higher functionality and a score zero suggests no functional role of a SNP 
Appendix Table 17: Bioinformatic analysis of genetic risk markers for the top one hundred age-specific associations with childhood ALL with the highest effect sizes $(P<0.05)$

\begin{tabular}{|c|c|c|c|c|c|c|c|}
\hline SNP & Chr & $\underset{\text { score }^{\dagger}}{\text { RegulomeDB }}$ & F-SNP ${ }^{* *}$ & Gene & $\begin{array}{c}\text { Correlated } \\
\text { SNP }\end{array}$ & $\underset{\text { score }^{\dagger}}{\text { RegulomeDB }}$ & $\begin{array}{c}\text { Gene } \\
\text { (correlated SNP) }\end{array}$ \\
\hline rs17109582 & 1 & 5 & NS & DIO1 & rs76114672 & $2 b$ & DIO1-HSPB11 \\
\hline rs17109582 & 1 & 5 & NS & DIO1 & rs77840912 & $2 b$ & HSPB11 \\
\hline rs7304215 & 12 & NS & NS & TMEM132D & - & - & . \\
\hline rs10999147* & 10 & 4 & 0.31 & AIFM2 & . & . & . \\
\hline rs7330286 & 13 & 6 & NS & LINC00343-RNA5SP38 & . & . & . \\
\hline rs41309927 & 20 & 7 & NS & SEC23B & • & - & - \\
\hline rs36086854* & 4 & 4 & 0.07 & CFAP99 & . & . & . \\
\hline rs $17409162^{*}$ & 3 & 5 & 0.37 & ANO10 & rs9856765 & $2 a$ & ANO10-ABHD5 \\
\hline rs13129052 & 4 & 5 & 0.1 & RGS12 & rs994425 & $2 b$ & C4orf44-RGS12 \\
\hline rs13129052 & 4 & 5 & 0.1 & RGS12 & rs61211078 & $2 b$ & C4orf44-RGS12 \\
\hline rs9856456 & 3 & NS & 0.1 & LOC101926953-IGSF11 & . & . & . \\
\hline rs6927353 & 6 & 6 & NS & TCP10-LOC401286 & . & . & . \\
\hline rs $11651675^{*}$ & 17 & $2 \mathrm{a}$ & 0.5 & TMC8 & rs117626020 & $2 \mathrm{a}$ & TMC8 \\
\hline rs10109794 & 8 & $\mathbf{3 a}$ & NS & TRIM55 & rs112477862 & $2 b$ & LOC101060153 \\
\hline rs10099957 & 8 & 4 & NS & TRIM55 & rs7846184 & $2 b$ & DNAJC5B-TRIM55 \\
\hline rs10099957 & 8 & 4 & NS & TRIM55 & rs7843038 & $2 b$ & TRIM55 \\
\hline rs56291145* & 13 & 4 & NS & ATP12A & . & . & . \\
\hline rs2909644 & 18 & NS & NS & $\begin{array}{l}\text { RP11-146N18.1- } \\
\text { LOC101927404 }\end{array}$ & . & · & . \\
\hline rs6093579 & 20 & 6 & 0.27 & PTPRT & rs6093581 & $3 a$ & PTPRT \\
\hline rs17039265 & 1 & 5 & 0.27 & HNRNPCL1-PRAMEF2 & rs 138700801 & Missense & HNRNPCL1 \\
\hline
\end{tabular}


Appendix Table 17 continued....

\begin{tabular}{|c|c|c|c|c|c|c|c|}
\hline SNP & Chr & $\begin{array}{c}\text { RegulomeDB } \\
\text { score }^{\dagger}\end{array}$ & F-SNP ${ }^{* *}$ & Gene & $\begin{array}{c}\text { Correlated } \\
\text { SNP }\end{array}$ & $\begin{array}{c}\text { RegulomeDB } \\
\text { score }^{\dagger}\end{array}$ & $\begin{array}{c}\text { Gene } \\
\text { (correlated SNP) }\end{array}$ \\
\hline rs 17039265 & 1 & 5 & 0.27 & HNRNPCL1-PRAMEF2 & rs141872266 & Missense & HNRNPCL1 \\
\hline rs17039265 & 1 & 5 & 0.27 & HNRNPCL1-PRAMEF2 & rs41279492 & Missense & HNRNPCL1 \\
\hline rs17039265 & 1 & 5 & 0.27 & HNRNPCL1-PRAMEF2 & rs9728577 & Missense & PRAMEF2 \\
\hline rs9728577" & 1 & 6 & 0.27 & PRAMEF2 & . & . & . \\
\hline rs75411676 & 1 & NS & NS & PRAMEF2 & . & . & . \\
\hline rs36068434 & 4 & 5 & 0.1 & CDS1 & rs148100862 & $2 b$ & CDS1 \\
\hline rs36068434* & 4 & 5 & 0.1 & CDS1 & rs113914543 & $2 b$ & CDS1 \\
\hline rs11916525 & 3 & 5 & NS & CEP70 & rs12632793 & 1d & CEP70 \\
\hline rs11916525 & 3 & 5 & NS & CEP70 & rs12632794 & 1d & CEP70 \\
\hline rs11916525 & 3 & 5 & NS & CEP70 & rs9049 & 1f & CEP70 \\
\hline rs11916525 & 3 & 5 & NS & CEP70 & rs 1563615 & 1f & CEP70 \\
\hline rs3204798* & 1 & 6 & 0.91 & PRAMEF2 & . & . & . \\
\hline rs $35340237^{*}$ & 3 & NS & NS & CEP70 & rs35537868 & Missense & FAM62C \\
\hline rs8176018 & 7 & 4 & 0 & $K E L$ & rs7810229 & $2 b$ & KEL \\
\hline rs1835154 & 2 & $3 \mathbf{a}$ & NS & GALNT13-KCNJ3 & rs16837893 & $3 a$ & GALNT13-KCNJ3 \\
\hline rs78323957 & 8 & 4 & NS & SLC10A5 & rs16909527 & 1f & CHMP4C \\
\hline rs16909509* & 8 & NS & NS & ZFAND1 & rs16909527 & 1f & CHMP4C \\
\hline rs3729598 & 5 & 6 & 0.27 & LIFR & rs76127780 & $3 a$ & LIFR \\
\hline rs568760 & 15 & 5 & 0 & HCN4 & . & . & . \\
\hline rs1800141 & 12 & 4 & 0.27 & LRP1/MIR1228 & . & . & . \\
\hline rs35384751 ${ }^{* \dagger \dagger}$ & 5 & 4 & 0.64 & $F Y B$ & . & . & . \\
\hline rs11175471 & 12 & NS & 0 & RASSF3 & rs17223244 & $2 b$ & RASSF3 \\
\hline rs2389891 & 4 & NS & NS & $P D E 5 A$ & . & . & . \\
\hline
\end{tabular}


Appendix Table 17 continued....

\begin{tabular}{|c|c|c|c|c|c|c|c|}
\hline SNP & Chr & $\begin{array}{c}\text { RegulomeDB } \\
\text { score }^{\dagger}\end{array}$ & F-SNP ${ }^{* *}$ & Gene & $\begin{array}{l}\text { Correlated } \\
\text { SNP }\end{array}$ & $\begin{array}{c}\text { RegulomeDB } \\
\text { score }^{\dagger}\end{array}$ & $\begin{array}{c}\text { Gene } \\
\text { (correlated SNP) }\end{array}$ \\
\hline rs35424709* & 3 & 5 & 0.1 & ATP13A4 & . & . & . \\
\hline rs12188300 & 5 & 4 & NS & IL12B-LOC285627 & . & . & . \\
\hline rs7303641 & 12 & NS & 0 & RASSF3 & rs7959471 & $2 b$ & RASSF3 \\
\hline rs34139105* & 11 & NS & 0 & PRRG4 & . & . & . \\
\hline rs10500248 & 19 & NS & NS & $\begin{array}{c}\text { ZNF536- } \\
\text { LOC101927254 }\end{array}$ & . & . & . \\
\hline rs2124906 & 19 & NS & NS & $\begin{array}{c}\text { ZNF536- } \\
\text { LOC101927254 }\end{array}$ & . & . & . \\
\hline rs7492685 & 14 & 6 & NS & RPL18P1-ATP5G2P2 & . & . & . \\
\hline rs 276725 & 19 & NS & NS & $\begin{array}{c}\text { ZNF536- } \\
\text { LOC101927254 }\end{array}$ & . & . & . \\
\hline rs6851586 & 4 & 5 & 0.1 & ANAPC4-LOC645433 & . & . & . \\
\hline rs16871434 & 4 & 6 & 0.1 & KCNIP4 & . & . & . \\
\hline rs28421666 & 6 & $3 \mathbf{a}$ & NS & $\begin{array}{c}\text { HLA-DRB1-HLA- } \\
\text { DQA1 }\end{array}$ & rs2395226 & $3 a$ & $\begin{array}{l}\text { HLA-DRB1- } \\
\text { HLA-DQA1 }\end{array}$ \\
\hline rs 17055478 & 13 & NS & NS & $\begin{array}{c}\text { DNAJA1P1- } \\
\text { HMGN2P39 }\end{array}$ & . & . & . \\
\hline rs 1582274 & 7 & NS & NS & IMMP2L- DOCK4 & rs75469463 & $3 a$ & IMMP2L-DOCK4 \\
\hline rs6572051 & 14 & 6 & NS & LOC644919 & . & . & . \\
\hline rs9407838 & 9 & 6 & 0.5 & SH3GL2 & . & . & . \\
\hline rs72838683* & 10 & 4 & NS & PDZD7 & . & . & . \\
\hline rs11250832 & 10 & 6 & NS & ADARB2- LINC00700 & . & . & . \\
\hline rs16827743 & 3 & 5 & 0.1 & $\begin{array}{l}\text { LOC101926953- } \\
\text { LOC101926968 }\end{array}$ & . & . & . \\
\hline rs12705300 & 7 & 4 & NS & SRPK2 & . & . & . \\
\hline rs1915164 & 15 & NS & NS & SQRDL-LOC729316 & . & . & . \\
\hline rs12083795 & 1 & 5 & 0.1 & POU3F1- RRAGC & rs72663642 & $2 a$ & POU3F1- RRAGC \\
\hline
\end{tabular}


Appendix Table 17 continued....

\begin{tabular}{|c|c|c|c|c|c|c|c|}
\hline SNP & Chr & $\begin{array}{c}\text { RegulomeDB } \\
\text { score }^{\dagger}\end{array}$ & F-SNP ${ }^{* * *}$ & Gene & $\begin{array}{l}\text { Correlated } \\
\text { SNP }\end{array}$ & $\begin{array}{c}\text { RegulomeDB } \\
\text { score }^{\dagger}\end{array}$ & $\begin{array}{c}\text { Gene } \\
\text { (correlated SNP) }\end{array}$ \\
\hline rs9861022 & 3 & 4 & 0.1 & LOC101926953-IGSF11 & . & . & . \\
\hline rs16964079 & 15 & 6 & NS & C15orf41/CSNK1A1P1 & rs28659969 & $3 a$ & C15orf41 \\
\hline rs16964079 & 15 & 6 & NS & C15orf41/CSNK1A1P1 & rs28480404 & $3 a$ & C15orf41 \\
\hline rs16964079 & 15 & 6 & NS & C15orf41/CSNK1A1P1 & rs 28629456 & $3 a$ & C15orf41 \\
\hline rs16964079 & 15 & 6 & NS & C15orf41/CSNK1A1P1 & rs12903299 & $3 a$ & C15orf41 \\
\hline rs16964079 & 15 & 6 & NS & C15orf41/CSNK1A1P1 & rs77483951 & $3 \mathrm{a}$ & CSNK1A1P1 \\
\hline rs16964079 & 15 & 6 & NS & C15orf41/CSNK1A1P1 & rs8031162 & $3 a$ & C15orf41 \\
\hline rs16964079 & 15 & 6 & NS & C15orf41/CSNK1A1P1 & rs12595212 & $3 \mathrm{a}$ & CSNK1A1P1 \\
\hline rs 10157510 & 1 & 4 & NS & VAMP4-METTL13 & rs10913576 & $2 b$ & VAMP4 \\
\hline rs10224856 & 7 & NS & NS & VPS41 & rs2214668 & $2 \mathrm{~b}$ & VPS41 \\
\hline rs 1728850 & 15 & 5 & NS & UNC13C-RSL24D1 & rs 183003270 & $3 a$ & UNC13C-RSL24D1 \\
\hline rs 1728850 & 15 & 5 & NS & UNC13C-RSL24D1 & rs139840085 & $3 a$ & UNC13C-RSL24D1 \\
\hline rs17055548 & 13 & 6 & NS & $\begin{array}{l}\text { DNAJA1P1- } \\
\text { HMGN2P39 }\end{array}$ & - & . & • \\
\hline rs10133677 & 14 & $3 \mathbf{a}$ & 0 & MDGA2 & . & . & . \\
\hline rs3130161 & 6 & 5 & 0.5 & HLA-DPB1-COL11A2 & rs3129203 & $2 b$ & $\begin{array}{c}\text { HLA-DPB1- } \\
\text { COL11A2 }\end{array}$ \\
\hline rs 1000521 & 14 & NS & NS & SLC8A3 & rs8011824 & $2 \mathrm{~b}$ & SLC8A3 \\
\hline rs 1000521 & 14 & NS & NS & SLC8A3 & rs17107699 ${ }^{\dagger \dagger}$ & $2 \mathrm{~b}$ & SLC8A3 \\
\hline rs 12257453 & 10 & 6 & NS & ARMC3 & . & . & . \\
\hline rs11833839 & 12 & 6 & NS & EP400 & rs1127023 & $2 b$ & EP400 \\
\hline rs3790126 & 16 & 4 & 0.18 & EMP2 & rs12598331 & $2 a$ & ATF7IP2-EMP2 \\
\hline rs16950438 & 16 & 5 & NS & LINC01571-C16orf97 & . & . & . \\
\hline
\end{tabular}


Appendix Table 17 continued....

\begin{tabular}{|c|c|c|c|c|c|c|c|}
\hline SNP & Chr & $\begin{array}{c}\text { RegulomeDB } \\
\text { score }^{\dagger}\end{array}$ & F-SNP ${ }^{* *}$ & Gene & $\begin{array}{c}\text { Correlated } \\
\text { SNP }\end{array}$ & $\begin{array}{c}\text { RegulomeDB } \\
\text { score }^{\dagger} \\
\end{array}$ & $\begin{array}{c}\text { Gene } \\
\text { (correlated SNP) }\end{array}$ \\
\hline rs6151599 & 5 & 4 & 0.27 & MSH3/DHFR & rs11749051 & $3 a$ & DHFR \\
\hline rs4546429 & 5 & 5 & 0.1 & LINC00603-PTGER4 & . & . & . \\
\hline rs11234604 & 11 & $2 \mathbf{b}$ & NS & C11orf73 & . & . & . \\
\hline rs6708453 & 2 & 5 & 0.1 & TEKT4P3-TRNAE26 & rs55872863 & $3 a$ & IMP4 \\
\hline rs3805114 & 3 & 5 & 0.24 & $A B C C 5$ & . & . & . \\
\hline rs9328207 & 6 & 5 & 0.5 & TDGF1P4-LOC728344 & . & . & . \\
\hline rs 2864680 & 5 & NS & NS & FSTL4 & . & . & . \\
\hline rs4741086 & 9 & NS & NS & DMRT2-RPS27AP14 & . & . & . \\
\hline rs 10514074 & 18 & NS & NS & $\begin{array}{l}\text { LOC100505817- } \\
\text { FBX015 }\end{array}$ & . & . & . \\
\hline rs41285557* & 5 & 4 & NS & MAML1 & . & . & . \\
\hline rs7620914 & 3 & NS & 0.1 & LOC101928135 & . & . & . \\
\hline rs7091901 & 10 & 5 & NS & ZNF438-ZEB1 & rs 10826908 & $2 c$ & $\begin{array}{c}\text { ZNF438- } \\
\text { LOC100129789 }\end{array}$ \\
\hline rs8100295 & 19 & 4 & NS & ZNF331-DPRX & . & . &. \\
\hline rs $17407084^{*}$ & 9 & 5 & 0.56 & $A K 8$ & rs 73550647 & $2 a$ & $A K 8$ \\
\hline rs222314 & 7 & NS & NS & LOC340268-HSPA8P8 & . & . & . \\
\hline rs7770942 & 6 & $2 b$ & NS & $\begin{array}{c}\text { EXOC2- } \\
\text { LOC101927691 }\end{array}$ & rs6900976 & $2 b$ & EXOC2-LOC285768 \\
\hline rs16916188* & 8 & 5 & 0.5 & $R B M 12 B$ & . & . & . \\
\hline rs17140942 & 16 & NS & 0.5 & RBFOX1 & . & . & . \\
\hline rs7172316 & 15 & $2 \mathbf{b}$ & NS & NPM1P42- NEO1 & . & . & . \\
\hline rs1209145 & 14 & 5 & NS & C14orf105- SLC35F4 & rs373690 & 1f & C14orf105-SLC35F4 \\
\hline rs 10902652 & 1 & NS & 0.1 & SNRPEP7-WDTC1 & . & . & . \\
\hline rs 1457759 & 5 & NS & 0.1 & LOC101927041- KCNN2 & . & . & . \\
\hline
\end{tabular}


Appendix Table 17 continued....

\begin{tabular}{|c|c|c|c|c|c|c|c|}
\hline SNP & Chr & $\begin{array}{c}\text { RegulomeDB } \\
\text { score }^{\dagger}\end{array}$ & F-SNP ${ }^{* *}$ & Gene & $\begin{array}{c}\text { Correlated } \\
\text { SNP } \\
\end{array}$ & $\begin{array}{c}\text { RegulomeDB } \\
\text { score }^{\dagger}\end{array}$ & $\begin{array}{c}\text { Gene } \\
\text { (correlated SNP) }\end{array}$ \\
\hline rs10826912 & 10 & 5 & NS & ZNF438-ZEB1 & rs10826908 & $2 \mathrm{c}$ & ZNF438-ZEB1 \\
\hline rs61744122 ${ }^{*}$ & 16 & NS & NS & VWAЗA & . & . & . \\
\hline rs4748309 & 10 & 6 & NS & RSU1 & . & . & . \\
\hline rs8013602 & 14 & 6 & NS & HNRNPUP1-KRT8P2 & . & & . \\
\hline rs9560160 & 13 & 5 & NS & $\begin{array}{l}\text { LOC102724489- } \\
\text { LOC102724510 }\end{array}$ & . & . & . \\
\hline rs 1755588 & 1 & 4 & 0.07 & ACOT11 & rs 1655527 & $2 b$ & ACOT11 \\
\hline rs 1755588 & 1 & 4 & 0.07 & ACOT11 & rs114205182 & $2 b$ & ACOT11 \\
\hline rs 1755588 & 1 & 4 & 0.07 & ACOT11 & rs116752923 & $2 b$ & ACOT11 \\
\hline rs 1755588 & 1 & 4 & 0.07 & ACOT11 & rs41313244 & $2 \mathrm{~b}$ & ACOT11 \\
\hline rs 1755588 & 1 & 4 & 0.07 & ACOT11 & rs60613980 & $2 b$ & ACOT11 \\
\hline rs $10493958^{\dagger \dagger}$ & 1 & $3 \mathbf{a}$ & NS & LOC102723784-OLFM3 & rs12724739 & $3 a$ & $\begin{array}{c}\text { LOC102723784- } \\
\text { OLFM3 }\end{array}$ \\
\hline rs7169431 & 15 & 5 & NS & NEDD4-RFX7 & . & . & . \\
\hline rs $17170286^{\dagger \dagger}$ & 7 & 5 & 0.50 & BBS9 & . & . & . \\
\hline rs11945730 & 4 & $3 \mathbf{a}$ & 0.27 & BST1-CD38 & . & . & . \\
\hline rs 17149645 & 11 & NS & NS & $\begin{array}{c}\text { LOC100506368- } \\
\text { TMEM135 }\end{array}$ & . & . & . \\
\hline
\end{tabular}

Note: Some associated SNPs showed multiple correlated functional variants

$\mathrm{Chr}=$ Chromosome; NS $=$ No score; SNP = single nucleotide polymorphism; $*$ Missense; $* *$ Nonsense; ${ }^{\dagger}$ SNPs change or create a binding site for transcription factors

${ }^{\dagger}$ RegulomDb predicts score from 1 to 7 . A score of one suggests SNPs have higher functionality and may play an important role in gene regulation. A score of 7 predicts least functionality

${ }^{* * *}$ F-SNP predicts score from 0 to 1 . A score of one suggest higher functionality and a score zero suggests no functional role of a SNP 
Appendix Table 18: Expression quantitative trait locus analysis of age-specific associated genetic risk variants

\begin{tabular}{|c|c|c|c|c|}
\hline SNP & Chr & Feature & Gene & eQTL \\
\hline rs $7320982^{*}$ & 13 & intergenic & MIRHG1-GPC5 & ADM, GNB4 \\
\hline rs2212824* & 21 & intergenic & ADAMTS5-GPXP2 & CRIPAK, MTG1 \\
\hline rs $2051713^{*}$ & 12 & intergenic & MRPL2P1-C12orf37 & $H 1 F X$ \\
\hline rs6519091* & 22 & intron & MICALL1 & $L M F 1, M Y D 88$ \\
\hline rs2899293* & 22 & intron & ANKRD54 & LMF1 \\
\hline rs $139866^{*}$ & 22 & intron & POLR2F & CNPY3 \\
\hline rs6510588* & 19 & intron & ZNF420 & ZNF420 \\
\hline rs $1209145^{*}$ & 14 & intergenic & C14orf105-SLC35F4 & CCR10 \\
\hline rs973358* & 17 & intron & SHISA6 & WDTC1 \\
\hline rs $7952853^{*}$ & 12 & intron & OR6C2 & PAG1 \\
\hline rs877111* & 6 & intergenic & C6orf223 & MRPL14 \\
\hline rs $4821723^{*}$ & 22 & 5' upstream & MICALL1 & LMF1 \\
\hline rs $2562599^{*}$ & 19 & intergenic & ZNF568-ZNF420 & SLC13A2, ZNF420 \\
\hline rs735482* & 19 & 3'UTR & ERCC1/CD3EAP & HDGFL1, ERCC1 \\
\hline rs $4714739^{*}$ & 6 & intergenic & C6orf223-MRPL14 & MRPL14 \\
\hline rs $4714755^{*}$ & 6 & intron & TMEM63B & MRPL14 \\
\hline rs1321097* & 6 & intergenic & C6orf223-MRPL14 & MRPL14 \\
\hline rs910616 & 6 & intergenic & C6orf223-MRPL14 & MRPL14 \\
\hline rs4711767* & 6 & intron & MRPL14 & MRPL14 \\
\hline rs9462964* & 6 & intron & TMEM63B & MRPL14 \\
\hline rs $1935611^{*}$ & 6 & intron & MRPL14 & MRPL14 \\
\hline rs $4714741^{*}$ & 6 & intergenic & C6orf223-MRPL14 & MRPL14 \\
\hline rs $7770652^{*}$ & 6 & intron & MRPL14 & MRPL14 \\
\hline rs $7304215^{* *}$ & 12 & intron & TMEM132D & BCR, FLJ42953, LOC728468 \\
\hline rs $10514074^{* *}$ & 18 & intergenic & $\begin{array}{l}\text { LOC100505817- } \\
\quad \text { FBXO15 }\end{array}$ & $\begin{array}{c}\text { ZC3H7A, KIF5B, CORO1A, } \\
\text { LOC606724, APRT, STX10, } \\
\text { ARHGEF1, AGPAT9, CHTF18, } \\
\text { C16orf24 }\end{array}$ \\
\hline rs1209145 ${ }^{* *}$ & 14 & intergenic & C14orf105- SLC35F4 & CCR10 \\
\hline
\end{tabular}

$\mathrm{eQTL}=$ expression quantitative trait loci; $\mathrm{Chr}=$ chromosome

*Age-specific results based on P value; **Age-specific results based on effect size 


\section{Appendix Table 19: Genes showing age-specific associations (ranked by their $P$ values) and their interactions with chemicals in studies curated in the Comparative Toxicological Database}

\begin{tabular}{|c|c|c|c|}
\hline Gene/region & SNP & Chemical interaction & PUBMED ID \\
\hline SOX5 & rs 10505918 & $\begin{array}{c}\text { arsenite, benzo(a)pyrene, benzo(a)pyrene diol epoxide, } \\
\text { bisphenol A, copper sulfate, acetaminophen, carbon } \\
\text { tetrachloride, diethylhexyl phthalate, ethinyl estradiol, } \\
\text { methylmercury compounds, PCB, potassium } \\
\text { dichromate, propiconazole, tetrachlorodibenzodioxin, } \\
\text { tobacco smoke pollution }\end{array}$ & $\begin{array}{c}23974009,22316170,20382639,20170705, \\
19549813,17562736,17484886,19850644, \\
17555576,20020106,20020106,23608068, \\
21570461,17942748,20133372\end{array}$ \\
\hline HNF4G & rs11997355* & $\begin{array}{c}\text { acetaminophen, aflatoxin B1, air pollutants, } \\
\text { benzo(a)pyrene, formaldehyde, } \\
\text { tetrachlorodibenzodioxin, lead acetate, } \\
\text { diethylnitrosamine, chlordane }\end{array}$ & $\begin{array}{l}22230336,20106945,21632981,23649840, \\
21632981,22609695,22129741,20403969\end{array}$ \\
\hline ZFHX4 & rs11997355* & $\begin{array}{l}\text { atrazine, benzo(a)pyrene, benzo(a)pyrene diol epoxide, } \\
\text { carbon tetrachloride, diethylnitrosamine, } \\
\text { propiconazole, tobacco smoke pollution }\end{array}$ & $\begin{array}{c}22378314,22316170,20382639,17484886, \\
22129741,21278054,20133372\end{array}$ \\
\hline $\operatorname{IRX} 3$ & $\begin{array}{l}\text { rs } 2388773^{*} \\
\text { rs } 1151277^{*} \\
\text { rs } 1882591^{*} \\
\text { rs } 1493897^{*}\end{array}$ & $\begin{array}{c}\text { estradiol, coppoer sulfate, formaldehyde, smoke, } \\
\text { tetrachlorodibenzodioxin, aflatoxin B1, } \\
\text { benzo(a)pyrene, caffeine, nicotine, nitrofen }\end{array}$ & $\begin{array}{c}21185374,19549813,20655997,21095227, \\
21632981,19770486,22228805,20230807, \\
20230807,21238641\end{array}$ \\
\hline CHRM3 & rs665159 & $\begin{array}{l}\text { lead, silicon dioxide, testosterone, PCB, aflatoxin B1, } \\
\text { ammonium chloride, carbon tetrachloride, chlorpyrifos, } \\
\text { diazinon, dibutyl phthalate, dichlorvos, dimethoate, } \\
\text { disulfoton, environmental pollutants, chlorinated } \\
\text { hydrocarbons, methyl parathion, potassium dichromate, } \\
\text { progesterone, trinitrotoluene }\end{array}$ & $\begin{array}{c}19921347,23806026,21592394,19114083, \\
23630614,16483693,16644059,17666426, \\
16207942,15620428,19100812,15033006, \\
22872703,22872703,17666426,23608068, \\
15900516,21346803\end{array}$ \\
\hline$R F X 3$ & rs3954950* & $\begin{array}{l}\text { arsenic, atrazine, diazinon, formaldehyde, potassium } \\
\text { chromate(VI), PCB, benzo(a)pyrene }\end{array}$ & $\begin{array}{c}16835338,22378314,22964155,23649840, \\
22079256,19114083,19770486\end{array}$ \\
\hline GLIS3 & rs3954950* & $\begin{array}{c}\text { asbestos, benzo(a)pyrene, estradiol, potassium } \\
\text { chromate(VI), carbon tetrachloride }\end{array}$ & $\begin{array}{c}17331233,20106945,20106945,22079256, \\
17484886\end{array}$ \\
\hline RNF122 & rs $7818844^{*}$ & benzo(a)pyrene, dibutyl phthalate & 22228805,21266533 \\
\hline NPSR1 & $\begin{array}{l}\mathrm{rs} 6972158 \\
\mathrm{rs} 10250709\end{array}$ & $\begin{array}{l}\text { atrazine, cadmium, lead acetate, } \\
\text { tetrachlorodibenzodioxin }\end{array}$ & $22378314,24376830,22641619,21570461$ \\
\hline
\end{tabular}


Appendix Table 19 continued....

\begin{tabular}{|c|c|c|c|}
\hline Gene/region & SNP & Chemical interaction & PUBMED ID \\
\hline GPC5 & rs $7320982 *$ & $\begin{array}{l}\text { PCB, atrazine, benzo(a)pyrene, cocaine, } \\
\text { diethylnitrosamine }\end{array}$ & $\begin{array}{c}23829299,22378314,20106945,18355967, \\
22129741\end{array}$ \\
\hline GPC5 & rs7320982* & $\begin{array}{c}\text { PCB, atrazine, benzo(a)pyrene, cocaine, } \\
\text { diethylnitrosamine }\end{array}$ & $\begin{array}{c}23829299,22378314,20106945,18355967, \\
22129741\end{array}$ \\
\hline KHDRBS3 & $\begin{array}{l}\text { rs7016929* } \\
\text { rs1868368* }\end{array}$ & $\begin{array}{c}\text { acetaminophen, cobaltous chloride, } \\
\text { tetrachlorodibenzodioxin, PCB, aflatoxin B1, } \\
\text { benzo(a)pyrene, carbon tetrachloride }\end{array}$ & $\begin{array}{c}21420995,19320972,22574217,19114083, \\
19770486,19770486\end{array}$ \\
\hline MSH3 & $\begin{array}{l}\text { rs } 10079494^{*} \\
\quad \text { rs } 26784\end{array}$ & $\begin{array}{l}\text { benzo(a)pyrene, copper sulfate, } \\
\text { tetrachlorodibenzodioxin, tobacco smoke pollution, } \\
\text { PCB, aflatoxin B1, cadmium chloride, propiconazole }\end{array}$ & $\begin{array}{l}21632981,19549813,11007951,22623965, \\
19114083,19770486,11958953,21278054\end{array}$ \\
\hline$R A S G R F 2$ & rs $10079494 *$ & $\begin{array}{c}\text { arsenite, copper sulfate, PCB, acetaminophen, } \\
\text { benzo(a)pyrene, dibutyl phthalate, tobacco smoke } \\
\text { pollution }\end{array}$ & $\begin{array}{c}23974009,19549813,19114083,17585979, \\
21839799,18087596,21266533\end{array}$ \\
\hline WNT8A & rs $7720650 *$ & bisphenol A & 21786754 \\
\hline NME5 & rs $7720650 *$ & PCB, tetrachlorodibenzodioxin & 21334430,19933214 \\
\hline GPR85 & $\begin{array}{l}\text { rs2949827* } \\
\text { rs2396653* }\end{array}$ & $\begin{array}{c}\text { carbon tetrachloride, chlorpyrifos, copper sulfate, } \\
\text { tetrachlorodibenzodioxin }\end{array}$ & $17484886,18668222,18579281,21570461$ \\
\hline$P P P 1 R 3 A$ & $\begin{array}{l}\text { rs } 2949827 * \\
\text { rs } 2396653 *\end{array}$ & acetaminophen, bisphenol A, copper sulfate & $17562736,17706920,18579281$ \\
\hline CD97 & rs202050948 & $\begin{array}{l}\text { aflatoxin B1, arsenic trioxide, benzo(a)pyrene, } \\
\text { estradiol, copper sulfate, hydrogen peroxide, lithium } \\
\text { chloride, PCB, 2,6-dinitrotoluene, acetaminophen, } \\
\text { tetrachlorodibenzodioxin, toluene }\end{array}$ & $\begin{array}{l}20106945,19128835,20064835,23019147, \\
19549813,20044591,23527032,19114083, \\
21346803,17562736,24680724,22967744\end{array}$ \\
\hline MATN3 & rs1191818* & $\begin{array}{l}\text { aflatoxin B1, atrazine, benzo(a)pyrene, copper sulfate, } \\
\text { diuron, ethanol, fonofos, hydrogen peroxide, parathion, } \\
\text { terbufos, testosterone, tetrachlorodibenzodioxin }\end{array}$ & $\begin{array}{l}21632981,22378314,21632981,19549813, \\
24172598,20621659,22847954,20044591, \\
22847954,22847954,21592394,21632981\end{array}$ \\
\hline LAPTM4A & rs1191818* & $\begin{array}{c}\text { atrazine, potassium dichromate, lipopolysaccharides, } \\
\text { pentachlorophenol, Tetrachlorodibenzodioxin }\end{array}$ & $\begin{array}{c}22378314,23718831,10975854,23892564, \\
21570461\end{array}$ \\
\hline GNMT & rs3800292 & $\begin{array}{c}\text { aflatoxin B1, benzo(a)pyrene, estradiol, copper sulfate, } \\
\text { dioxins, methylcholanthrene, testosterone, } \\
\text { tetrachlorodibenzodioxin, acetaminophen, carbon } \\
\text { tetrachloride, lipopolysaccharide, } \\
\text { tetrachlorodibenzodioxin }\end{array}$ & $\begin{array}{l}20106945,22100608,20106945,19549813, \\
22100608,22100608,21592394,22298810, \\
17562736,18346771,18346771,16214954\end{array}$ \\
\hline
\end{tabular}


Appendix Table 19 continued....

\begin{tabular}{|c|c|c|c|}
\hline Gene/region & SNP & Chemical interaction & PUBMED ID \\
\hline C7orf50 & rs1881116 & arsenic, benzene, benzo(a)pyrene, cobaltous chloride & $18414638,19162166,21632981,19320972$ \\
\hline GCAT & rs $4821717^{*}$ & $\begin{array}{l}\text { copper sulfate, diazinon, tetrachlorodibenzodioxin, } 2,4- \\
\text { dinitrotoluene, acetaminophen, aflatoxin B1, } \\
\text { benzo(a)pyrene, carbon tetrachloride, dibutyl phthalate, } \\
\text { testosterone, tetrachlorodibenzodioxin }\end{array}$ & $\begin{array}{c}19549813,22964155,21632981,21346803 \\
17562736,19770486,23735875,17484886, \\
17361019,20403060,19770486\end{array}$ \\
\hline GALR3 & rs $4821717^{*}$ & $\begin{array}{l}\text { aflatoxin B1, lead, methylmercuric chloride, PCB, } \\
\text { acetaminophen }\end{array}$ & $\begin{array}{c}22100608,19921347,21385734,19114083 \\
17562736\end{array}$ \\
\hline ADAMTS5 & rs2212824* & $\begin{array}{l}\text { acetaminophen, arsenic trioxide, benzo(a)pyrene diol } \\
\text { epoxide, fonofos, parathion, particulate matter, } \\
\text { potassium chromate(VI), terbufos, } \mathrm{PCB} \text {, aflatoxin } \mathrm{B} 1 \text {, } \\
\text { carbon tetrachloride, diuron, tetrachlorodibenzodioxin }\end{array}$ & $\begin{array}{c}21420995,25258189,20382639,22847954, \\
22847954,23085030,22079256,22847954, \\
23196670,23630614,17484886,25152437, \\
19465110\end{array}$ \\
\hline INSM2 & rs $7157104^{*}$ & atrazine, copper sulfate, tetrachlorodibenzodioxin & $22378314,19549813,21354282$ \\
\hline GFRA2 & rs $2125649^{*}$ & $\begin{array}{l}\text { atrazine, cobaltous chloride, } \mathrm{PCB} \text {, carbon tetrachloride, } \\
\text { chlorpyrifos, cocaine, paraquat, progesterone }\end{array}$ & $\begin{array}{l}22378314,17553155,19114083,17805973 \\
17452286,12358776,18198484,22238285\end{array}$ \\
\hline TRIB2 & rs6739633* & $\begin{array}{c}\text { acetaminophen, arsenic trioxide, asbestos, atrazine, } \\
\text { benzo(a)pyrene diol epoxide, estradiol, formaldehyde, } \\
\text { nicotine, progesterone, smoke, } \\
\text { tetrachlorodibenzodioxin, benzo(a)pyrene, dibutyl } \\
\text { phthalate, lead acetate, paraquat, potassium dichromate }\end{array}$ & $\begin{array}{l}21420995,20458559,17331233,22378314, \\
20382639,14699072,23649840,16949557, \\
20660070,21095227,21296121,22610609, \\
21266533,21829687,21371552,23608068\end{array}$ \\
\hline FAM84A & rs6739633* & $\begin{array}{c}\text { acetaminophen, benzo(a)pyrene, estradiol, dibutyl } \\
\text { phthalate, propiconazole }\end{array}$ & $\begin{array}{c}17562736,19770486,19484750,21266533, \\
21278054\end{array}$ \\
\hline MAP2K4 & rs $237320^{*}$ & $\begin{array}{l}\text { arsenic trioxide, benzo(a)pyrene diol epoxide, } \\
\text { estradiol, cadmium chloride, hydrogen peroxide, } \\
\text { lipopolysaccharides, acetaminophen, cocaine, } \\
\text { diethylnitrosamine, lipopolysaccharides, } \\
\text { methylmercury compounds, tetrachlorodibenzodioxin, } \\
\text { tobacco smoke pollution }\end{array}$ & $\begin{array}{c}14701702,19150397,23094148,18703135, \\
16707465,21112663,17562736,18355967, \\
18691550,21548916,19666049,21570461, \\
15289447\end{array}$ \\
\hline MYOCD & rs $237320^{*}$ & $\begin{array}{l}\text { asbestos, benzo(a)pyrene, benzo(a)pyrene diol epoxide, } \\
\text { fonofos, parathion, progesterone, terbufos, PCB, } \\
\text { tetrachlorodibenzodioxin, tobacco smoke pollution }\end{array}$ & $\begin{array}{c}18687144,22178795,, 20382639,22847954, \\
22847954,20864642,22847954,19114083, \\
17942748,20133372\end{array}$ \\
\hline SOX10 & rs139886 & lead, diethylnitrosamine, progesterone & $19921347,20360939,17478888$ \\
\hline
\end{tabular}


Appendix Table 19 continued....

\begin{tabular}{|c|c|c|c|}
\hline Gene/region & SNP & Chemical interaction & PUBMED ID \\
\hline$A G A$ & rs $13116233^{*}$ & $\begin{array}{c}\text { cobaltous chloride, fonofos, parathion, terbufos, } 2,6- \\
\text { dinitrotoluene, acetaminophen, benzo(a)pyrene, dibutyl } \\
\text { phthalate, vehicle emissions }\end{array}$ & $\begin{array}{c}19376846,22847954,22847954,22847954, \\
21346803,17562736,21715664,21266533, \\
19165385\end{array}$ \\
\hline$M I C A L L 1$ & rs6519091 & $\begin{array}{c}\text { acetaminophen, aflatoxin B1, benzo(a)pyrene, } \\
\text { estradiol, cobaltous chloride, formaldehyde, } \\
\text { methylmercuric chloride }\end{array}$ & $\begin{array}{c}22230336,20106945,21632981,20106945, \\
19320972,23649840,23179753\end{array}$ \\
\hline SPTLC3 & rs6109487* & $\begin{array}{l}\text { acetaminophen, aflatoxin B1, air pollutants, } \\
\text { benzo(a)pyrene, copper sulfate, formaldehyde, } \\
\text { nicotine, PCB, tetrachlorodibenzodioxin }\end{array}$ & $\begin{array}{c}21420995,20106945,21757418,22316170, \\
19549813,23649840,23825647,21334430, \\
21632981\end{array}$ \\
\hline$S L C 23 A 2$ & rs6052937 & $\begin{array}{c}\text { acetaminophen, air pollutants, arsenite, } \\
\text { benzo(a)pyrene, estradiol, cobaltous chloride, } \\
\text { progesterone, tetrachlorodibenzodioxin, carbon } \\
\text { tetrachloride, iron, ozone, paraquat, propiconazole, } \\
\text { toluene }\end{array}$ & $\begin{array}{c}21420995,21757418,23974009,21632981, \\
17092984,19376846,17092984,21632981, \\
17484886,16629162,20417186,22558179, \\
22334560,18653662\end{array}$ \\
\hline NEK1 & rs34099167 & $\begin{array}{c}\text { acetaminophen, arsenic trioxide, benzo(a)pyrene, } \\
\text { cobaltous chloride, copper sulfate, hydrogen peroxide, } \\
\text { potassium chromate(VI) }\end{array}$ & $\begin{array}{c}22230336,22521957,20064835,19376846, \\
19549813,18951874,22079256\end{array}$ \\
\hline ATP6AP1L & rs $7722067 *$ & aflatoxin B1, benzo(a)pyrene & 23630614,21839799 \\
\hline$C L N K$ & rs 1004327 & potassium dichromate, tetrachlorodibenzodioxin & 23608068,21570461 \\
\hline TMTC2 & rs3993401* & $\begin{array}{l}\text { atrazine, benzo(a)pyrene, formaldehyde, hydrogen } \\
\text { peroxide, lead, methylcholanthrene, smoke, } \\
\text { tetrachlorodibenzodioxin, acetaminophen, aflatoxin } \\
\text { B1, arsenic, dibutyl phthalate, tobacco smoke pollution }\end{array}$ & $\begin{array}{c}22378314,22316170,23649840,18951874, \\
19921347,20348232,21095227,22903824, \\
17562736,19770486,19654921,21266533, \\
20133372\end{array}$ \\
\hline ZC3H13 & rs $2806907 *$ & $\begin{array}{l}\text { formaldehyde, acetaminophen, diuron, ethanol, } \\
\text { tetrachlorodibenzodioxin }\end{array}$ & $\begin{array}{c}23649840,17562736,25152437,17618662, \\
21889950\end{array}$ \\
\hline NID2 & $\begin{array}{l}\text { rs2101919 } \\
\text { rs3825594 }\end{array}$ & $\begin{array}{l}\text { air pollutants, arsenates, arsenite, atrazine, } \mathrm{PCB}, \\
\text { benzo(a)pyrene, estradiol, carbon tetrachloride, } \\
\text { diethylnitrosamine, diuron, testosterone, } \\
\text { tetrachlorodibenzodioxin }\end{array}$ & $\begin{array}{c}21757418,18585445,23974009,18585445, \\
23196670,22228805,19484750,17484886, \\
18164116,20032058,21570461\end{array}$ \\
\hline ANKRD54 & rs 2899293 & $\begin{array}{l}\text { copper sulfate, aflatoxin B1, dibutyl phthalate, nickel } \\
\text { monoxide, tetrachlorodibenzodioxin }\end{array}$ & $\begin{array}{c}19549813,19770486,21266533,19167457, \\
21570461\end{array}$ \\
\hline
\end{tabular}


Appendix Table 19 continued....

\begin{tabular}{|c|c|c|c|}
\hline Gene/region & SNP & Chemical interaction & PUBMED ID \\
\hline SPATA13 & rs1028695 & $\begin{array}{l}\text { arsenic trioxide, benzo(a)pyrene diol epoxide, } \\
\text { estradiol, copper sulfate, aflatoxin B1, ciguatoxins, } \\
\text { dibutyl phthalate, propiconazole }\end{array}$ & $\begin{array}{c}20458559,19150397,17962382,19549813, \\
19770486,18353800,21266533,21278054\end{array}$ \\
\hline PCSK5 & rs2185230 & $\begin{array}{l}\text { PCB, arsenic trioxide, benzene, benzo(a)pyrene, } \\
\text { estradiol, copper sulfate, progesterone, } \\
\text { tetrachlorodibenzodioxin, 2,4-dinitrotoluene, } \\
\text { acetaminophen, aflatoxin B1, carbon tetrachloride, } \\
\text { dibutyl phthalate, diuron, propiconazole }\end{array}$ & $\begin{array}{c}19692669,15761015,19162166,22316170, \\
20660070,19549813,20660070,22298810, \\
21346803,17585979,19770486,17484886, \\
21266533,25152437,21278054\end{array}$ \\
\hline ZNF420 & rs4254428 & arsenite, formaldehyde & 23974009,20655997 \\
\hline POLR2F & rs139866 & $\begin{array}{c}\text { cobaltous chloride, copper sulfate, diazinon, hydrogen } \\
\text { peroxide, PCB, 2,6-dinitrotoluene, dibutyl phthalate, } \\
\text { tetrachlorodibenzodioxin }\end{array}$ & $\begin{array}{l}19376846,19549813,22964155,18951874, \\
19114083,21346803,21266533,21570461\end{array}$ \\
\hline$E L F$ & rs5028798* & copper sulfate & 16973896 \\
\hline$E H F$ & rs $5028798 *$ & $\begin{array}{l}\text { arsenite, asbestos, estradiol, formaldehyde, } \\
\text { progesterone, smoke, tetrachlorodibenzodioxin, } \\
\text { aflatoxin B1, furan, tobacco smoke pollution }\end{array}$ & $\begin{array}{c}23974009,17331233,20660070,23649840, \\
20660070,21095227,23152189,23630614, \\
22079235,20133372\end{array}$ \\
\hline TMC5 & rs 1894381 & $\begin{array}{l}\text { air pollutants, arsenic trioxide, benzo(a)pyrene diol } \\
\text { epoxide, copper sulfate, hydrogen peroxide, smoke, } \\
\text { tetrachlorodibenzodioxin, tobacco smoke pollution }\end{array}$ & $\begin{array}{l}21757418,20707922,20018196,19549813, \\
18951874,21095227,21570461,20133372\end{array}$ \\
\hline FGF12 & rs9809647* & $\begin{array}{c}\text { aflatoxin B1, arsenic, arsenic trioxide, arsenite, } \\
\text { fonofos, parathion, potassium chromate(VI), terbufos, } \\
\text { uranium compounds }\end{array}$ & $\begin{array}{c}22100608,24675094,22521957,23974009, \\
22847954,22847954,22079256,22847954, \\
19654044\end{array}$ \\
\hline POU3F1 & rs $898982 *$ & $\begin{array}{c}\text { diazinon, lead, PCB, acetaminophen, arsenite, } \\
\text { diethylnitrosamine, diuron, potassium dichromate, } \\
\text { tetrachlorodibenzodioxin }\end{array}$ & $\begin{array}{c}22964155,19921347,24046277,17562736, \\
18929588,18164116,25152437,23608068, \\
22298810\end{array}$ \\
\hline$R R A G C$ & rs $898982 *$ & $\begin{array}{c}\text { acetaminophen, asbestos, benzene, cobaltous chloride, } \\
\text { copper sulfate, formaldehyde, benzo(a)pyrene, } \\
\text { tetrachlorodibenzodioxin }\end{array}$ & $\begin{array}{c}22230336,18687144,19162166,19376846, \\
19549813,23649840,20504355,21570461\end{array}$ \\
\hline EXOC2 & rs9392857* & $\begin{array}{l}\text { benzo(a)pyrene, potassium chromate(VI), PCB, 2,4- } \\
\text { dinitrotoluene, cadmium chloride, dibutyl phthalate, } \\
\text { diethylnitrosamine, tetrachlorodibenzodioxin }\end{array}$ & $\begin{array}{l}22316170,22079256,19114083,21346803, \\
22457795,21266533,23968726,21570461\end{array}$ \\
\hline
\end{tabular}


Appendix Table 19 continued....

\begin{tabular}{|c|c|c|c|}
\hline Gene/region & SNP & Chemical interaction & PUBMED ID \\
\hline BRMS1L & rs8014810 & $\begin{array}{l}\text { acetaminophen, aflatoxin B1, benzo(a)pyrene, } \\
\text { benzo(a)pyrene diol epoxide, formaldehyde, dibutyl } \\
\text { phthalate, particulate matter, pentachlorophenol }\end{array}$ & $\begin{array}{l}22230336,21641981,22316170,19150397, \\
23649840,21266533,19176365,23892564\end{array}$ \\
\hline RALGAPA1 & $\begin{array}{l}\text { rs } 8021355 \\
\text { rs7144565 } \\
\text { rs2274068 }\end{array}$ & $\begin{array}{c}\text { arsenic trioxide, benzo(a)pyrene, benzo(a)pyrene diol } \\
\text { epoxide, copper sulfate, hydrogen peroxide, mercury, } \\
\text { 2,6-dinitrotoluene, acetaminophen, lead acetate, } \\
\text { sodium arsenate }\end{array}$ & $\begin{array}{c}20458559,20064835,19150397,19549813, \\
21179406,19937285,21346803,17562736, \\
20542052,21795629\end{array}$ \\
\hline ERCC1 & rs762562 & $\begin{array}{c}\text { aflatoxin B1, arsenic, asbestos, benzene, } \\
\text { benzo(a)pyrene diol epoxide, benzo(a)pyrene, } \\
\text { estradiol, cobaltous chloride, copper sulfate, } \\
\text { lipopolysaccharides, paraquat, 2,4-dinitrotoluene, } \\
\text { acetaminophen, benzo(a)pyrene, carbon tetrachloride, } \\
\text { furan, lead nitrate, particulate matter, } \\
\text { tetrachlorodibenzodioxin, vehicle emissions }\end{array}$ & $\begin{array}{l}22100608,16882524,20855422,16188086, \\
23203453,20624995,22082530,19376846, \\
19549813,22777745,23613995,21346803, \\
17562736,22641617,15056808,22079235, \\
11891201,12919963,21570461,12919963\end{array}$ \\
\hline CDЗЕАР & rs 762562 & $\begin{array}{l}\text { aflatoxin B1, cobaltous chloride, formaldehyde, } \\
\text { hydrogen peroxide, acetaminophen, benzo(a)pyrene }\end{array}$ & $\begin{array}{c}22100608,19320972,23649840,20044591, \\
17562736,21715664\end{array}$ \\
\hline GRB10 & rs4948088* & $\begin{array}{l}\text { arsenic trioxide, benzo(a)pyrene, benzo(a)pyrene diol } \\
\text { epoxide, estradiol, cobaltous chloride, copper sulfate, } \\
\text { progesterone, PCB, aflatoxin B1, carbon tetrachloride, } \\
\text { chlorpyrifos, diuron, lead acetate, particulate matter, } \\
\text { testosterone, vehicle emissions }\end{array}$ & $\begin{array}{l}20458559,22316170,20382639,14699072, \\
19320972,19549813,21795739,19114083, \\
19770486,17484886,20350560,25152437, \\
25270620,21222557,20403060,21222557\end{array}$ \\
\hline$C O B L$ & rs4948088* & $\begin{array}{l}\text { acetaminophen, aflatoxin B1, benzo(a)pyrene, } \\
\text { benzo(a)pyrene diol epoxide, estradiol, copper sulfate, } \\
\text { smoke, caffeine, copper sulfate, diuron, } \\
\text { lipopolysaccharides, propiconazole, } \\
\text { tetrachlorodibenzodioxin }\end{array}$ & $\begin{array}{c}22230336,20106945,22316170,20018196, \\
23019147,19549813,21095227,20864626, \\
18579281,21551480,12057914,21278054, \\
19933214\end{array}$ \\
\hline MARK2 & rs3850942 & $\begin{array}{l}\text { copper sulfate, 2,4-dinitrotoluene, 2,6-dinitrotoluene, } \\
\text { acetaminophen, propiconazole }\end{array}$ & $\begin{array}{c}19549813,21346803,21346803,17562736, \\
22228805,16730040\end{array}$ \\
\hline TNFAIPBL & rs2899469* & aflatoxin $\mathrm{B} 1$, benzo(a)pyrene, tetrachlorodibenzodioxin & $22100608,20064835,21889950$ \\
\hline
\end{tabular}


Appendix Table 19 continued....

\begin{tabular}{|c|c|c|c|}
\hline Gene/region & SNP & Chemical interaction & PUBMED ID \\
\hline CYP19A1 & rs2899469* & $\begin{array}{l}\text { PCB, aflatoxin B1, atrazine, benzo(a)pyrene, estradiol, } \\
\text { bisphenol A, cadmium, chlordan, copper sulfate, } \\
\text { dicofol, endosulfan, estrogens, flusilazole, heptachlor, } \\
\text { hexaconazole, Ibuprofen, lipopolysaccharides, } \\
\text { methoxychlor, progesterone, propiconazole, } \\
\text { testosterone, tetrachlorodibenzodioxin }\end{array}$ & $\begin{array}{l}16216300,23402939,11675267,24361490, \\
12839940,22120136,24376830,16996190, \\
19549813,10806373,16996190,16054421, \\
15554355,16996190,12127262,15964185, \\
10657851,22120136,22130322,16996190, \\
12628686,19147701\end{array}$ \\
\hline MDGA2 & $\begin{array}{l}\text { rs } 8011343 \\
\text { rs } 10133677 \\
\text { rs } 10133677\end{array}$ & $\begin{array}{l}\text { sodium arsenite, benzo(a)pyrene, } \\
\text { tetrachlorodibenzodioxin }\end{array}$ & $21925251,22228805,21570461$ \\
\hline SOX1 & rs2031293* & $\begin{array}{c}\text { benzo(a)pyrene, hydralazine, dibutyl phthalate, } \\
\text { tetrachlorodibenzodioxin }\end{array}$ & $22316170,17183730,21266533,24680724$ \\
\hline CPNE8 & rs11171543* & $\begin{array}{l}\text { benzo(a)pyrene, benzo(a)pyrene diol epoxide, } \\
\text { potassium chromate(VI), smoke, } \\
\text { tetrachlorodibenzodioxin, acetaminophen, cocaine, } \\
\text { tetrachlorodibenzodioxin }\end{array}$ & $\begin{array}{l}21632981,20018196,22079256,21095227, \\
21632981,17562736,17898221,21570461\end{array}$ \\
\hline KIF21A & $\begin{array}{l}\text { rs11171543* } \\
\text { rs11172024 }\end{array}$ & $\begin{array}{c}\text { aspirin, benzo(a)pyrene diol epoxide, estradiol, } \\
\text { cobaltous chloride, hydrogen peroxide, 2,6- } \\
\text { dinitrotoluene, benzo(a)pyrene, } \\
\text { tetrachlorodibenzodioxin }\end{array}$ & $\begin{array}{l}11906190,19150397,20404318,19320972, \\
21179406,21346803,22228805,21570461\end{array}$ \\
\hline INPP4B & $\begin{array}{l}\operatorname{rs} 1510136^{*} \\
\operatorname{rs} 6834625^{*}\end{array}$ & $\begin{array}{l}\text { atrazine, benzene, benzo(a)pyrene, benzo(a)pyrene diol } \\
\text { epoxide, estradiol, progesterone, } \\
\text { tetrachlorodibenzodioxin, 2,4-dinitrotoluene, 2,6- } \\
\text { dinitrotoluene, paraquat }\end{array}$ & $\begin{array}{c}22378314,19162166,22316170,20382639, \\
23019147,21795739,20106945,21346803, \\
21346803,18198484\end{array}$ \\
\hline USP38 & $\begin{array}{l}\text { rs } 1510136^{*} \\
\text { rs6834625* }\end{array}$ & $\begin{array}{c}\text { atrazine, benzo(a)pyrene diol epoxide, cobaltous } \\
\text { chloride, copper sulfate, formaldehyde, 2,6- } \\
\text { dinitrotoluene }\end{array}$ & $\begin{array}{c}22378314,20018196,19320972,19549813, \\
20655997,21346803\end{array}$ \\
\hline ADAMTS5 & $\begin{array}{l}\text { rs } 16979806^{*} \\
\text { rs377621* } \\
\text { rs } 2830698^{*}\end{array}$ & $\begin{array}{l}\text { acetaminophen, arsenic trioxide, benzo(a)pyrene diol } \\
\text { epoxide, fonofos, parathion, particulate matter, } \\
\text { potassium chromate(VI), terbufos, PCB, aflatoxin B1, } \\
\text { carbon tetrachloride, diuron, tetrachlorodibenzodioxin }\end{array}$ & $\begin{array}{c}21420995,25258189,20382639,22847954, \\
22847954,23085030,22079256,22847954, \\
23196670,23630614,17484886,25152437, \\
19465110\end{array}$ \\
\hline CCSER1 & rs 17017488 & cocaine & 18438686 \\
\hline
\end{tabular}


Appendix Table 19 continued....

\begin{tabular}{|c|c|c|c|}
\hline Gene/region & SNP & Chemical interaction & PUBMED ID \\
\hline MAMDC2 & rs 10511980 & $\begin{array}{l}\text { benzo(a)pyrene, benzo(a)pyrene diol epoxide, } \\
\text { progesterone, acetaminophen, carbon tetrachloride, } \\
\text { diuron, lead acetate, tetrachlorodibenzodioxin }\end{array}$ & $\begin{array}{c}22316170,20382639,21795739,17562736, \\
17484886,25152437,22641619,19654925\end{array}$ \\
\hline SLC16A7 & rs2620870* & $\begin{array}{l}\text { acetaminophen, air pollutants, benzo(a)pyrene diol } \\
\text { epoxide, estradiol, copper sulfate, progesterone, smoke, } \\
\text { tetrachlorodibenzodioxin, 2,4-dinitrotoluene, } \\
\text { acetaminophen, aflatoxin B1, ethanol, heptachlor, } \\
\text { hydrogen peroxide, tetrachlorodibenzodioxin }\end{array}$ & $\begin{array}{c}21420995,21757418,20018196,19619570, \\
19549813,21795739,21095227,19619570, \\
21346803,17562736,19770486,19167417, \\
23153324,16766717,20702594\end{array}$ \\
\hline USP15 & rs2620870* & $\begin{array}{l}\text { aspirin, cobaltous chloride, formaldehyde, PCB, 2,6- } \\
\text { dinitrotoluene, acetaminophen, benzo(a)pyrene, } \\
\text { tetrachlorodibenzodioxin }\end{array}$ & $\begin{array}{l}11906190,19376846,23649840,21334430, \\
21346803,17942131,15034205,15034205\end{array}$ \\
\hline TLR4 & rs $10739501 *$ & $\begin{array}{c}\text { PCB, aflatoxin B1, arsenic, lipopolysaccharides, } \\
\text { progesterone, smoke, tobacco smoke pollution, } \\
\text { acetaminophen, benzo(a)pyrene, carbon tetrachloride, } \\
\text { ethanol, ozone, paraquat, particulate matter, } \\
\text { tetrachlorodibenzodioxin, tobacco smoke pollution, } \\
\text { vehicle emissions }\end{array}$ & $\begin{array}{c}21703328,22100608,19945496,22014880, \\
22014880,22516759,24269501,17562736, \\
22228805,17484886,16439473,17626835, \\
23969119,12832678,21653731,24612634, \\
20362647\end{array}$ \\
\hline BRINP1 & rs $10739501 *$ & arsenic trioxide, $\mathrm{PCB}$ & 15761015,23196670 \\
\hline C14orf105 & rs $1209145^{*}$ & $\begin{array}{c}\text { acetaminophen, aflatoxin B1, arsenic trioxide, } \\
\text { benzo(a)pyrene, formaldehyde, } \\
\text { tetrachlorodibenzodioxin }\end{array}$ & $\begin{array}{c}21420995,20106945,15761015,20106945, \\
23649840,20106945\end{array}$ \\
\hline SLC35F4 & rs $1209145^{*}$ & acetaminophen, potassium dichromate & 17562736,23608068 \\
\hline NCOA2 & rs $1481046^{*}$ & $\begin{array}{l}\text { aflatoxin B1, air pollutants, arsenic trioxide, } \\
\text { benzo(a)pyrene, estradiol, bisphenol A, } \\
\text { lipopolysaccharides, potassium chromate(VI), } \\
\text { tetrachlorodibenzodioxin, furan, lead acetate, } \\
\text { lipopolysaccharides, progesterone }\end{array}$ & $\begin{array}{c}20106945,21757418,19131511,21632981, \\
17962382,23583298,21370964,22079256, \\
18842620,22079235,22641619,16847310, \\
17556502\end{array}$ \\
\hline TRAM1 & rs $1481046^{*}$ & $\begin{array}{c}\text { benzo(a)pyrene diol epoxide, copper sulfate, PCB, } \\
\text { estradiol }\end{array}$ & $20018196,19549813,19114083,20068009$ \\
\hline
\end{tabular}


Appendix Table 19 continued....

\begin{tabular}{|c|c|c|c|}
\hline Gene/region & SNP & Chemical interaction & PUBMED ID \\
\hline PTPN14 & rs 11120342 & $\begin{array}{l}\text { cobaltous chloride, copper sulfate, } \mathrm{PCB} \text {, } \\
\text { benzo(a)pyrene, dibutyl phthalate, diuron, lead acetate, } \\
\text { ozone, tetrachlorodibenzodioxin }\end{array}$ & $\begin{array}{c}19320972,19549813,19114083,21569818, \\
21266533,25152437,25270620,17095637, \\
21570461\end{array}$ \\
\hline$V S X 1$ & rs6037033* & $\begin{array}{c}\text { acetaminophen, arsenite, copper sulfate, hydrogen } \\
\text { peroxide, aflatoxin B1 }\end{array}$ & $\begin{array}{c}22230336,23974009,19549813,18951874, \\
23630614\end{array}$ \\
\hline ENTPD6 & rs6037033* & acetaminophen & 21420995 \\
\hline MYSM1 & rs12073211* & $\begin{array}{l}\text { formaldehyde, potassium chromate(VI), } \\
\text { tetrachlorodibenzodioxin, 2,4-dinitrotoluene, 2,6- } \\
\text { dinitrotoluene, benzo(a)pyrene, propiconazole, } \\
\text { tetrachlorodibenzodioxin }\end{array}$ & $\begin{array}{l}20655997,22079256,22298810,21346803, \\
21346803,19770486,21278054,21570461\end{array}$ \\
\hline$J U N$ & rs $12073211 *$ & $\begin{array}{l}\text { PCB, aflatoxin B1, air pollutants, arsenic trioxide, } \\
\text { arsenite, asbestos, aspirin, atrazine, benzene, } \\
\text { benzo(a)pyrene, benzo(a)pyrene diol epoxide, } \\
\text { estradiol, cadmium chloride, cocaine, copper sulfate, } \\
\text { diquat, endosulfan, heptachlor, hydrogen peroxide, } \\
\text { lipopolysaccharides, nicotine, paraquat, particulate } \\
\text { matter, potassium dichromate, } \\
\text { tetrachlorodibenzodioxin }\end{array}$ & $\begin{array}{c}16611624,22100608,21757418,18633430, \\
22260869,18687144,16600694,22378314, \\
17119257,16041517,20382639,15930183, \\
11341981,15814102,19549813,21172416, \\
22146149,15888667,8853906,24149798, \\
11592233,16237197,22178795,20493934, \\
15019843\end{array}$ \\
\hline$R U N X 3$ & rs $742390^{*}$ & $\begin{array}{l}\text { arsenite, benzo(a)pyrene, estradiol, diazinon, fonofos, } \\
\text { parathion, terbufos, tetrachlorodibenzodioxin, tobacco } \\
\text { smoke pollution, 4-amino-2,6-dinitrotoluene, } \\
\text { acetaminophen }\end{array}$ & $\begin{array}{l}23974009,20064835,20404318,22964155, \\
22847954,22847954,22847954,22298810, \\
23724145,21346803,17562736\end{array}$ \\
\hline SYF2 & rs 742390* & $\begin{array}{c}\text { atrazine, copper sulfate, formaldehyde, potassium } \\
\text { chromate(VI), PCB, acetaminophen, benzo(a)pyrene, } \\
\text { tetrachlorodibenzodioxin }\end{array}$ & $\begin{array}{c}22378314,19549813,23649840,22079256, \\
17562736,22228805,24680724\end{array}$ \\
\hline SHISA6 & rs973358 & Tretinoin & 21934132 \\
\hline
\end{tabular}


Appendix Table 19 continued....

\begin{tabular}{|c|c|c|c|}
\hline Gene/region & SNP & Chemical interaction & PUBMED ID \\
\hline AGO2 & rs9694342 & $\begin{array}{c}\text { benzo(a)pyrene diol epoxide, estradiol, copper sulfate, } \\
\text { diazinon, formaldehyde, } 2,4 \text {-dinitrotoluene, } \\
\text { acetaminophen, cocaine, mercuric chloride, } \\
\text { tetrachlorodibenzodioxin }\end{array}$ & $\begin{array}{c}20018196,23019147,19549813,22964155, \\
20655997,21346803,17562736,20643829, \\
16507785,21570461\end{array}$ \\
\hline ZFAND2A & rs6943934* & $\begin{array}{l}\text { acetaminophen, benzo(a)pyrene, benzo(a)pyrene diol } \\
\text { epoxide, estradiol, cobaltous chloride, copper sulfate, } \\
\text { diazinon, formaldehyde, potassium chromate(VI), } \\
\text { tobacco smoke pollution, aflatoxin B1, arsenite, } \\
\text { bisphenol A, dibutyl phthalate, } \\
\text { tetrachlorodibenzodioxin }\end{array}$ & $\begin{array}{l}21420995,21632981,19150397,20106945, \\
19320972,19549813,22964155,23649840, \\
22079256,22363418,19770486,16973439, \\
21932408,21266533,21570461\end{array}$ \\
\hline TRIB2 & rs $1521244 *$ & $\begin{array}{c}\text { acetaminophen, arsenic trioxide, asbestos, atrazine, } \\
\text { benzo(a)pyrene diol epoxide, estradiol, formaldehyde, } \\
\text { hydralazine, nicotine, progesterone, smoke, } \\
\text { tetrachlorodibenzodioxin, benzo(a)pyrene, dibutyl } \\
\text { phthalate, lead acetate, paraquat }\end{array}$ & $\begin{array}{l}21420995,20458559,17331233,22378314, \\
20382639,14699072,23649840,17183730, \\
16949557,20660070,21095227,21296121, \\
22610609,21266533,21829687,21371552\end{array}$ \\
\hline EFCAB5 & rs9897794 & potassium dichromate, tetrachlorodibenzodioxin & 23608068,21570461 \\
\hline C6orf223 & rs877111 & acetaminophen, formaldehyde & 22230336,23649840 \\
\hline MKI67 & rs753712 & $\begin{array}{l}\text { acetaminophen, aflatoxin B1, atrazine, benzene, } \\
\text { benzo(a)pyrene diol epoxide, estradiol, copper sulfate, } \\
\text { methylmercuric chloride, PCB, progesterone, } \\
\text { benzo(a)pyrene, bisphenol A, carbon tetrachloride, } \\
\text { diazinon, diethylnitrosamine, propiconazole, asbestos }\end{array}$ & $\begin{array}{c}22230336,20106945,18585445,21843810, \\
21776270,19167446,19549813,23179753, \\
21334430,16888808,21569818,22875908, \\
17484886,22546817,24012840,21278054, \\
17200189\end{array}$ \\
\hline TRPA1 & $\begin{array}{l}\text { rs } 1430838 \\
\text { rs2116078 }\end{array}$ & $\begin{array}{c}\text { benzene, benzo(a)pyrene diol epoxide, coal ash, dibutyl } \\
\text { phthalate, formaldehyde, ozone, particulate matter, } \\
\text { smoke }\end{array}$ & $\begin{array}{l}21591660,20382639,22155782,22935519, \\
21591660,20008466,23541125,23541125\end{array}$ \\
\hline ATRNL1 & rs 2615874 & $\begin{array}{l}\text { atrazine, estradiol, fonofos, parathion, terbufos, } \\
\text { tetrachlorodibenzodioxin }\end{array}$ & $\begin{array}{c}22378314,19619570,22847954,22847954, \\
22847954,19619570\end{array}$ \\
\hline
\end{tabular}

$\mathrm{SNP}=$ single nucleotide polymorphism 


\section{Appendix Table 20: Genes showing age-specific associations (ranked by their OR) and their interactions with chemicals in studies curated in the Comparative Toxicological Database}

\begin{tabular}{|c|c|c|c|}
\hline Gene/region & SNP & Chemical interaction & PUBMED ID \\
\hline DIO1 & rs17109582 & $\begin{array}{c}\text { aflatoxin B1, benzo(a)pyrene, estradiol,copper sulfate, } \\
\text { tetrachlorodibenzodioxin, PCB, 2,4-dinitrotoluene, } \\
\text { acetaminophen, arsenic, cobaltous chloride, } \\
\text { diethylnitrosamine, ethanol, fipronil, furan, } \\
\text { hexachlorobenzene, lead acetate, methoxychlor, } \\
\text { pentachlorophenol }\end{array}$ & $\begin{array}{l}20106945,21871943,20106945, \\
19549813,22298810,15254343, \\
20959002,21346803,17562736, \\
18288313,21139344,17602206, \\
15353170,23962444,22079235, \\
15664263,22609695,7574712\end{array}$ \\
\hline TMEM132D & rs 7304215 & fonofos, parathion, terbufos, potassium dichromate & $\begin{array}{c}22847954,22847954,22847954, \\
23608068\end{array}$ \\
\hline AIFM2 & rs10999147 & $\begin{array}{l}\text { benzo(a)pyrene, cobaltous chloride, copper sulfate, } \\
\text { tetrachlorodibenzodioxin, cadmium, hydrogen peroxide }\end{array}$ & $\begin{array}{l}21632981,19320972,19549813 \\
24067728,14987999\end{array}$ \\
\hline$S E C 23 B$ & rs41309927 & atrazine, cobaltous chloride, 2,6-dinitrotoluene & $22378314,19376846,21346803$ \\
\hline ANO10 & rs 17409162 & $\begin{array}{l}\text { formaldehyde, testosterone, aflatoxin B1, dibutyl phthalate, } \\
\text { tetrachlorodibenzodioxin }\end{array}$ & $\begin{array}{l}23649840,21592394,19770486, \\
21266533,21570461\end{array}$ \\
\hline RGS12 & rs13129052 & $\begin{array}{l}\text { acetaminophen, benzo(a)pyrene, copper sulfate, aflatoxin } \\
\text { B1, benzo(a)pyrene, dibutyl phthalate, diethylnitrosamine, } \\
\text { ethinyl estradiol, propiconazole, tetrachlorodibenzodioxin }\end{array}$ & $\begin{array}{l}22230336,20064835,19549813, \\
19770486,22228805,21266533, \\
18164116,17108234,21278054, \\
21570461\end{array}$ \\
\hline IGSF11 & $\begin{array}{l}\text { rs9856456* } \\
\text { rs9861022* }\end{array}$ & $\begin{array}{l}\text { estradiol, PCB, acetaminophen, benzo(a)pyrene, } \\
\text { chlorpyrifos, diethylnitrosamine, tetrachlorodibenzodioxin }\end{array}$ & $\begin{array}{c}20404318,19114083,17562736, \\
19770486,18668222,17602206, \\
21570461\end{array}$ \\
\hline TMC8 & rs11651675 & arsenic, copper sulfate, tetrachlorodibenzodioxin & $18414638,19549813,21570461$ \\
\hline TRIM55 & $\begin{array}{l}\text { rs } 10109794 \\
\text { rs10099957 }\end{array}$ & methylcholanthrene, acetaminophen, coal ash, & $20348232,17562736,19000753$ \\
\hline ATP12A & rs56291145 & tetrachlorodibenzodioxin, estradiol, lead acetate, smoke & $\begin{array}{c}23152189,19484750,25270620, \\
23693141 \\
\end{array}$ \\
\hline PTPRT & rs6093579 & $\begin{array}{l}\text { fonofos, parathion, terbufos, acetaminophen, aflatoxin B1, } \\
\text { diethylnitrosamine }\end{array}$ & $\begin{array}{l}22847954,22847954,22847954, \\
17562736,23630614,22129741\end{array}$ \\
\hline
\end{tabular}




\begin{tabular}{|c|c|c|c|}
\hline Gene/region & SNP & Chemical interaction & PUBMED ID \\
\hline PRAMEF2 & $\begin{array}{l}\text { rs } 17039265^{*} \\
\text { rs9728577 } \\
\text { rs } 75411676 \\
\text { rs } 3204798\end{array}$ & aflatoxin B1 & 22100608 \\
\hline CDS1 & rs36068434 & $\begin{array}{c}\text { estradiol, nicotine, benzo(a)pyrene, } \\
\text { tetrachlorodibenzodioxin, PCB, 2,4-dinitrotoluene, } \\
\text { tetrachlorodibenzodioxin }\end{array}$ & $\begin{array}{l}19167446,23825647,22228805, \\
17942748,19114083,21346803, \\
21215274\end{array}$ \\
\hline CEP70 & $\begin{array}{l}\text { rs } 11916525 \\
\text { rs35340237 }\end{array}$ & $\begin{array}{l}\text { acetaminophen, aflatoxin B1, air pollutants, arsenic trioxide, } \\
\text { atrazine, Benzo(a)pyrene, benzo(a)pyrene diol epoxide, } \\
\text { copper sulfate, potassium chromate(VI), 3-dinitrobenzene }\end{array}$ & $\begin{array}{c}22230336,22100608,21757418, \\
20458559,22378314,21632981 \\
20018196,19549813,22079256 \\
21983209\end{array}$ \\
\hline KEL & rs8176018 & $\begin{array}{l}\text { formaldehyde, acetaminophen, potassium dichromate, } \\
\text { tetrachlorodibenzodioxin }\end{array}$ & $\begin{array}{c}23649840,17562736,23608068, \\
19465110\end{array}$ \\
\hline GALNT13 & rs $1835154^{*}$ & copper sulfate, PCB, estradiol & $19549813,19114083,19484750$ \\
\hline KCNJ3 & rs $1835154^{*}$ & benzo(a)pyrene, carbon tetrachloride, ethanol & $22316170,16644059,22164246$ \\
\hline SLC10A5 & rs78323957 & arsenic & 21291286 \\
\hline ZFAND1 & rs 16909509 & tetrachlorodibenzodioxin, acetaminophen, dibutyl phthalate & $21632981,17562736,21266533$ \\
\hline LIFR & rs3729598 & $\begin{array}{l}\text { aflatoxin B1, arsenic, asbestos, benzo(a)pyrene, } \\
\text { benzo(a)pyrene diol epoxide, estradiol, methylmercuric } \\
\text { chloride, PCBs, testosterone, tetrachlorodibenzodioxin, 2,6- } \\
\text { dinitrotoluene, acetaminophen, arsenite, carbon } \\
\text { tetrachloride, furan, lipopolysaccharides, maneb, paraquat, } \\
\text { progesterone }\end{array}$ & $\begin{array}{l}22100608,24675094,17297452, \\
21632981,20018196,21185374, \\
23179753,21334430,21592394, \\
20106945,21346803,17585979, \\
18929588,17484886,22079235, \\
17321000,22563483,22563483, \\
21770760\end{array}$ \\
\hline HCN4 & rs568760 & aflatoxin B1, diazinon, methoxychlor & $22100608,22964155,23303685$ \\
\hline$L R P 1$ & rs $1800141^{*}$ & $\begin{array}{l}\text { air pollutants, arsenic trioxide, copper sulfate, hydrogen } \\
\text { peroxide, 2,4-dinitrotoluene, 2,6-dinitrotoluene, } \\
\text { acetaminophen, aflatoxin B1, benzo(a)pyrene, estradiol, } \\
\text { dibutyl phthalate, diethylnitrosamine, lipopolysaccharide, } \\
\text { propiconazole, tetrachlorodibenzodioxin }\end{array}$ & $\begin{array}{l}21757418,22521957,19549813, \\
23410634,21346803,21346803, \\
17585979,19770486,2228805, \\
16977382,21266533,17942915, \\
16977382,21278054,21570461\end{array}$ \\
\hline
\end{tabular}




\begin{tabular}{|c|c|c|c|}
\hline Gene/region & SNP & Chemical interaction & PUBMED ID \\
\hline$F Y B$ & rs35384751 & $\begin{array}{c}\text { arsenic trioxide, benzo(a)pyrene, cobaltous chloride, diuron, } \\
\text { fonofos, parathion, terbufos, acetaminophen, } \\
\text { tetrachlorodibenzodioxin }\end{array}$ & $\begin{array}{l}15761015,20064835,19376846, \\
24172598,22847954,22847954, \\
22847954,17562736,20702594\end{array}$ \\
\hline RASSF3 & $\begin{array}{l}\text { rs } 11175471 \\
\text { rs } 7303641\end{array}$ & $\begin{array}{c}\text { acetaminophen, benzo(a)pyrene, carbon tetrachloride, } \\
\text { tetrachlorodibenzodioxin }\end{array}$ & $\begin{array}{c}17562736,19770486,17484886, \\
21570461\end{array}$ \\
\hline PDE5A & rs $2389891 *$ & $\begin{array}{l}\text { benzo(a)pyrene, benzo(a)pyrene diol epoxide, estradiol, } \\
\text { PCB, caffeine, carbon tetrachloride, chlorpyrifos, diazinon, } \\
\text { methylmercuric chloride, testosterone, } \\
\text { tetrachlorodibenzodioxin, tobacco smoke pollution }\end{array}$ & $\begin{array}{l}22316170,20382639,22574217, \\
23196670,20864626,16644059, \\
17452286,17452286,20864626, \\
17138653,24680724,20133372\end{array}$ \\
\hline ATP13A4 & rs35424709 & $\begin{array}{l}\text { hydralazine, aflatoxin B1, diethylnitrosamine, } \\
\text { tetrachlorodibenzodioxin, tobacco smoke pollution }\end{array}$ & $\begin{array}{c}17183730,19770486,17942915 \\
19770486,20133372\end{array}$ \\
\hline$I L 12 B$ & rs12188300* & $\begin{array}{l}\text { estradiol, lipopolysaccharides, progesterone, } \mathrm{PCB} \text {, } \\
\text { acetaminophen, aflatoxin B1, benzene, benzo(a)pyrene, } \\
\text { bisphenol A, diazinon, diethylnitrosamine, ethanol, } \\
\text { formaldehyde, hydrogen peroxide, methoxychlor, nicotine, } \\
\text { ozone, particulate matter, progesterone, } \\
\text { tetrachlorodibenzodioxin, tobacco smoke pollution, vehicle } \\
\text { emissions }\end{array}$ & $\begin{array}{c}20130130,19211157,20130130, \\
19114083,22575169,22445859, \\
15894107,17275222,18569609, \\
17499902,25051504,20624996, \\
12161283,15723204,22842586, \\
21237301,18929643,19805405, \\
21977998,21097750,20133372, \\
19805405\end{array}$ \\
\hline PRRG4 & rs34139105 & $\begin{array}{l}\text { benzo(a)pyrene diol epoxide, formaldehyde, smoke, } \\
\text { tetrachlorodibenzodioxin, acetaminophen, aflatoxin B1, } \\
\text { benzo(a)pyrene, diethylnitrosamine }\end{array}$ & $\begin{array}{c}22101062,23649840,21095227, \\
22298810,17562736,23630614 \\
19770486,22129741\end{array}$ \\
\hline ANAPC4 & rs6851586* & $\begin{array}{l}\text { arsenite, potassium chromate(VI), 2,4-dinitrotoluene, 2,6- } \\
\text { dinitrotoluene, acetaminophen, benzo(a)pyrene, ethanol, } \\
\text { tetrachlorodibenzodioxin }\end{array}$ & $\begin{array}{l}23974009,22079256,21346803 \\
21346803,17562736,22228805 \\
17618662,21570461\end{array}$ \\
\hline KCNIP4 & rs16871434 & $\begin{array}{c}\text { benzo(a)pyrene, copper sulfate, furan, progesterone, } \\
\text { testosterone, tobacco smoke pollution }\end{array}$ & $\begin{array}{l}22316170,19549813,22079235, \\
22238285,19693291,19969065\end{array}$ \\
\hline HLA-DRB1 & rs28421666* & $\begin{array}{l}\text { arsenic, aspirin, benzo(a)pyrene, estradiol, lead, } \\
\text { progesterone, smoke }\end{array}$ & $\begin{array}{c}18414638,20485159,20106945, \\
20660070,19921347,20660070, \\
21095227\end{array}$ \\
\hline HLA-DQA1 & rs28421666* & $\begin{array}{l}\text { PCB, Arsenic, Benzene, Lipopolysaccharides, Mercury, } \\
\text { Progesterone, Aluminum }\end{array}$ & $\begin{array}{l}20638727,18414638,16188091, \\
22235868,19690047,21298039\end{array}$ \\
\hline
\end{tabular}




\begin{tabular}{|c|c|c|c|}
\hline Gene/region & SNP & Chemical interaction & PUBMED ID \\
\hline$I M M P 2 L$ & rs $1582274 *$ & $\begin{array}{l}\text { aflatoxin B1, benzo(a)pyrene, copper sulfate, formaldehyde, } \\
\text { acetaminophen, estradiol, tetrachlorodibenzodioxin }\end{array}$ & $\begin{array}{c}20106945,22316170,19549813, \\
23649840,17562736,19484750, \\
21570461\end{array}$ \\
\hline DOCK4 & rs $1582274 *$ & $\begin{array}{l}\text { aflatoxin } \mathrm{B} 1 \text {, asbestos, benzo(a)pyrene, benzo(a)pyrene diol } \\
\text { epoxide, diazinon, hydrogen peroxide, progesterone, } \\
\text { potassium dichromate, tetrachlorodibenzodioxin }\end{array}$ & $\begin{array}{l}20106945,18687144,21714911, \\
19150397,22964155,18951874, \\
21795739,23608068,19465110\end{array}$ \\
\hline SH3GL2 & rs9407838 & $\begin{array}{l}\text { aflatoxin B1, arsenic trioxide, benzo(a)pyrene, fonofos, } \\
\text { parathion, terbufos, tetrachlorodibenzodioxin, } \mathrm{PCB} \text {, dibutyl } \\
\text { phthalate, paraquat, potassium dichromate }\end{array}$ & $\begin{array}{l}20106945,15761015,20106945, \\
22847954,22847954,22847954, \\
20106945,21673325,21266533, \\
21371552,23608068\end{array}$ \\
\hline PDZD7 & rs72838683 & arsenic trioxide, copper sulfate & 20458559,19549813 \\
\hline$A D A R B 2$ & rs11250832* & $\begin{array}{c}\text { acetaminophen, aflatoxin B1, PCB, } \\
\text { tetrachlorodibenzodioxin }\end{array}$ & $\begin{array}{l}22230336,22100608,19114083, \\
21570461\end{array}$ \\
\hline SRPK2 & rs 12705300 & $\begin{array}{l}\text { acetaminophen, arsenite, benzo(a)pyrene, benzo(a)pyrene } \\
\text { diol epoxide, paraquat, potassium chromate(VI), 2,4- } \\
\text { dinitrotoluene, } 2,6 \text {-dinitrotoluene, aflatoxin B1, carbon } \\
\text { tetrachloride, chlorpyrifos, lipopolysaccharides, potassium } \\
\text { dichromate, propiconazole, toluene }\end{array}$ & $\begin{array}{l}22230336,23974009,20106945, \\
19150397,23613995,22079256, \\
21346803,21346803,19770486, \\
17484886,18668222,12057914, \\
23608068,21278054,22166486\end{array}$ \\
\hline$S Q R D L$ & rs 1915164* & $\begin{array}{l}\text { acetaminophen, benzo(a)pyrene, estradiol, copper sulfate, } \\
\text { hydrogen peroxide, potassium chromate(VI), progesterone, } \\
\text { tetrachlorodibenzodioxin, propiconazole }\end{array}$ & $\begin{array}{l}21420995,22316170,22574217 \\
19549813,23410634,22079256, \\
21795739,21632981,21278054\end{array}$ \\
\hline POU3F1 & rs12083795* & $\begin{array}{l}\text { diazinon, lead, PCB, acetaminophen, arsenite, } \\
\text { diethylnitrosamine, diuron, potassium dichromate, } \\
\text { tetrachlorodibenzodioxin }\end{array}$ & $\begin{array}{l}22964155,19921347,19114083 \\
17562736,18929588,18164116, \\
25152437,23608068,24046277\end{array}$ \\
\hline RRAGC & rs12083795* & $\begin{array}{l}\text { acetaminophen, asbestos, benzene, copper sulfate, } \\
\text { formaldehyde, benzo(a)pyrene, tetrachlorodibenzodioxin }\end{array}$ & $\begin{array}{c}22230336,18687144,19162166, \\
19549813,23649840,20504355, \\
21570461\end{array}$ \\
\hline C15orf41 & rs16964079 & potassium chromate(VI) & 22079256 \\
\hline VAMP4 & rs $10157510^{*}$ & $\begin{array}{c}\text { arsenic trioxide, cobaltous chloride, copper sulfate, } \\
\text { formaldehyde, aflatoxin B1, benzo(a)pyrene, nicotine, } \\
\text { tetrachlorodibenzodioxin }\end{array}$ & $\begin{array}{c}20458559,19376846,19549813, \\
23649840,19770486,15034205, \\
17997037,15034205 \\
\end{array}$ \\
\hline METTL13 & rs10157510* & $\begin{array}{c}\text { acetaminophen, atrazine, copper sulfate, } \\
\text { tetrachlorodibenzodioxin }\end{array}$ & $\begin{array}{c}21420995,22378314,19549813 \\
19654925\end{array}$ \\
\hline
\end{tabular}


Appendix Table 20 continued....

\begin{tabular}{|c|c|c|c|}
\hline Gene/region & SNP & Chemical interaction & PUBMED ID \\
\hline VPS41 & rs 10224856 & $\begin{array}{c}\text { atrazine, benzo(a)pyrene, bisphenol A, copper sulfate, } \\
\text { hydrogen peroxide, caffeine, dibutyl phthalate, nicotine, } \\
\text { tetrachlorodibenzodioxin }\end{array}$ & $\begin{array}{c}22378314,20064835,22576693, \\
19549813,21179406,21266533, \\
20230807,21570461\end{array}$ \\
\hline UNC13C & rs $1728850^{*}$ & formaldehyde, benzo(a)pyrene, furan, paraquat, testosterone & $\begin{array}{c}23649840,21839799,22079235, \\
22563483,20403060\end{array}$ \\
\hline RSL24D1 & rs $1728850 *$ & acetaminophen, benzo(a)pyrene diol epoxide, copper sulfate & $22230336,20018196,19549813$ \\
\hline MDGA2 & rs 10133677 & sodium arsenite, benzo(a)pyrene, tetrachlorodibenzodioxin & $21925251,22228805,21570461$ \\
\hline HLA-DPB1 & rs3130161* & benzene, benzo(a)pyrene, tetrachlorodibenzodioxin & $16188091,15034205,15034205$ \\
\hline COL11A2 & rs $3130161 *$ & copper sulfate, diuron, tetrachlorodibenzodioxin & $19549813,21551480,21570461$ \\
\hline SLC8A3 & rs 1000521 & PCB, bisphenol A, fonofos, parathion, terbufos, ethanol & $\begin{array}{l}23146750,23146750,22847954, \\
22847954,22847954,20655511\end{array}$ \\
\hline ARMC3 & rs 12257453 & benzo(a)pyrene & 22316170 \\
\hline EP400 & rs11833839 & $\begin{array}{c}\text { cobaltous chloride, copper sulfate, formaldehyde, 2,6- } \\
\text { dinitrotoluene, acetaminophen, benzo(a)pyrene, } \\
\text { propiconazole, tetrachlorodibenzodioxin }\end{array}$ & $\begin{array}{c}19320972,19549813,23649840, \\
21346803,17562736,20504355, \\
21278054,21570461\end{array}$ \\
\hline EMP2 & rs 3790126 & $\begin{array}{c}\text { acetaminophen, benzo(a)pyrene, benzo(a)pyrene diol } \\
\text { epoxide, estradiol, bisphenol A, copper sulfate, } \\
\text { progesterone, tetrachlorodibenzodioxin, PCB, dibutyl } \\
\text { phthalate, furan }\end{array}$ & $\begin{array}{c}22230336,22316170,20382639, \\
16474171,16474171,19549813, \\
20864642,22574217,19114083, \\
21266533,24183702\end{array}$ \\
\hline MSH3 & rs6151599 & $\begin{array}{c}\text { benzo(a)pyrene, copper sulfate, tetrachlorodibenzodioxin, } \\
\text { tobacco smoke pollution, PCB, aflatoxin B1, cadmium } \\
\text { chloride, propiconazole }\end{array}$ & $\begin{array}{c}21632981,19549813,11007951, \\
22623965,19114083,19770486, \\
11958953,21278054\end{array}$ \\
\hline$D H F R$ & rs6151599 & $\begin{array}{l}\text { acetaminophen, air pollutants, benzo(a)pyrene, } \\
\text { benzo(a)pyrene diol epoxide, estradiol, cobaltous chloride, } \\
\text { copper sulfate, formaldehyde, nicotine, potassium } \\
\text { chromate(VI), PCB, aflatoxin B1, arsenite, carbon } \\
\text { tetrachloride, dibutyl phthalate, diethylnitrosamine, } \\
\text { tetrachlorodibenzodioxin }\end{array}$ & $\begin{array}{l}22230336,21757418,20064835, \\
20018196,19167446,19376846, \\
19549813,23649840,12588870, \\
22079256,20005886,19770486, \\
18929588,17484886,21266533, \\
20360939,21570461\end{array}$ \\
\hline PTGER4 & rs4546429* & $\begin{array}{l}\text { benzo(a)pyrene, benzo(a)pyrene diol epoxide, estradiol, } \\
\text { cobaltous chloride, diuron, formaldehyde, particulate matter, } \\
\text { polychlorinated biphenyls, tetrachlorodibenzodioxin, } \\
\text { ammonium chloride, bisphenol A, carbon tetrachloride, } \\
\text { dibutyl phthalate, diuron, hydrazine, progesterone }\end{array}$ & $\begin{array}{l}22316170,20382639,19619570, \\
19320972,24172598,20655997, \\
22178795,21334430,19619570, \\
16483693,20096755,17484886, \\
15620428,21551480,15282401,\end{array}$ \\
\hline
\end{tabular}


Appendix Table 20 continued....

\begin{tabular}{|c|c|c|c|}
\hline Gene/region & SNP & Chemical interaction & PUBMED ID \\
\hline C11orf73 & rs11234604 & copper sulfate, potassium chromate(VI) & 19549813,22079256 \\
\hline$A B C C 5$ & rs3805114 & $\begin{array}{l}\text { acetaminophen, arsenic trioxide, aspirin, benzo(a)pyrene, } \\
\text { estradiol, estrogens, formaldehyde, hydrogen peroxide, } \\
\text { potassium chromate(VI), tetrachlorodibenzodioxin, PCB, } \\
\text { dibutyl phthalate, diethylnitrosamine, testosterone }\end{array}$ & $\begin{array}{l}17627974,20458559,21900887, \\
21871943,15072547,21233418, \\
23649840,21179406,22079256, \\
19619570,15833929,21266533, \\
17602206,20403060\end{array}$ \\
\hline FSTL4 & rs2864680 & arsenic trioxide, benzo(a)pyrene, potassium dichromate & $15894607,22228805,23608068$ \\
\hline DMRT2 & rs4741086* & $\begin{array}{l}\text { arsenite, cobalt, cobaltous chloride, fonofos, parathion, } \\
\text { terbufos, lead acetate, }\end{array}$ & $\begin{array}{c}23974009,20105288,20105288, \\
22847954,22847954,22847954, \\
21829687\end{array}$ \\
\hline FBXO15 & rs10514074* & aflatoxin B1, tetrachlorodibenzodioxin & 22100608,22298810 \\
\hline MAML1 & rs41285557 & $\begin{array}{l}\text { arsenic trioxide, asbestos, acetaminophen, benzo(a)pyrene, } \\
\text { propiconazole, tetrachlorodibenzodioxin }\end{array}$ & $\begin{array}{l}20458559,17331233,17562736, \\
22228805,21278054,21570461\end{array}$ \\
\hline ZNF438 & $\begin{array}{l}\text { rs } 7091901 * \\
\text { rs } 10826912 *\end{array}$ & cobaltous chloride, copper sulfate, potassium chromate(VI) & $20105288,19549813,22079256$ \\
\hline ZEB1 & $\begin{array}{l}\text { rs } 7091901 * \\
\text { rs } 10826912 *\end{array}$ & $\begin{array}{c}\text { acetaminophen, arsenic trioxide, benzo(a)pyrene diol } \\
\text { epoxide, estradiol, cobaltous chloride, copper sulfate, } \\
\text { estrogens, formaldehyde, progesterone, benzo(a)pyrene, } \\
\text { chlorpyrifos, diuron, mercuric chloride, } \\
\text { tetrachlorodibenzodioxin }\end{array}$ & $\begin{array}{l}21420995,20707922,19150397, \\
12154049,19376846,19549813, \\
18622689,23649840,20864642, \\
15034205,20350560,25152437, \\
16507785,15034205\end{array}$ \\
\hline ZNF331 & rs8100295* & arsenic, atrazine, benzene, lead & $\begin{array}{c}16835338,22378314,17119257, \\
19921347\end{array}$ \\
\hline$A K 8$ & rs 17407084 & benzo(a)pyrene, cadmium chloride & 22228805,22457795 \\
\hline EXOC2 & rs $7770942 *$ & $\begin{array}{l}\text { benzo(a)pyrene, potassium chromate(VI), PCB, 2,4- } \\
\text { dinitrotoluene, dibutyl phthalate, tetrachlorodibenzodioxin }\end{array}$ & $\begin{array}{l}22316170,22079256,19114083, \\
21346803,21266533,21570461\end{array}$ \\
\hline$R B M 12 B$ & rs16916188 & $\begin{array}{l}\text { atrazine, formaldehyde, aflatoxin } \mathrm{B} 1, \\
\text { tetrachlorodibenzodioxin }\end{array}$ & $\begin{array}{c}22378314,20655997,19770486, \\
21215274\end{array}$ \\
\hline RBFOX1 & rs 17140942 & PCB, dibutyl phthalate, tetrachlorodibenzodioxin & $19933214,17361019,19933214$ \\
\hline NEO1 & rs $7172316^{*}$ & $\begin{array}{l}\text { acetaminophen, atrazine, benzo(a)pyrene, estradiol, } \\
\text { hydrogen peroxide, progesterone, progesterone, } 2,4- \\
\text { dinitrotoluene, aflatoxin B1, carbon tetrachloride, cocaine, } \\
\text { dibutyl phthalate, formaldehyde, tetrachlorodibenzodioxin }\end{array}$ & $\begin{array}{l}22230336,22378314,21632981, \\
20660070,20044591,20660070, \\
20660070,21346803,19770486, \\
17484886,15056714,21266533, \\
12679049,21570461\end{array}$ \\
\hline
\end{tabular}


Appendix Table 20 continued....

\begin{tabular}{|c|c|c|c|}
\hline Gene/region & SNP & Chemical interaction & PUBMED ID \\
\hline C14orf105 & rs $1209145^{*}$ & $\begin{array}{l}\text { acetaminophen, aflatoxin B1, arsenic trioxide, } \\
\text { benzo(a)pyrene, formaldehyde, tetrachlorodibenzodioxin }\end{array}$ & $\begin{array}{l}21420995,20106945,15761015, \\
20106945,23649840,20106945\end{array}$ \\
\hline SLC35F4 & rs $1209145^{*}$ & acetaminophen, potassium dichromate & 17562736,23608068 \\
\hline WDTC1 & rs10902652* & $\begin{array}{l}\text { acetaminophen, diazinon, tetrachlorodibenzodioxin, } 2,4- \\
\text { dinitrotoluene, propiconazole, tetrachlorodibenzodioxin }\end{array}$ & $\begin{array}{l}22230336,22964155,22298810 \\
21346803,21278054,24680724\end{array}$ \\
\hline KCNN2 & rs1457759* & $\begin{array}{c}\text { arsenite, benzo(a)pyrene, benzo(a)pyrene diol epoxide, } \\
\text { testosterone, tetrachlorodibenzodioxin, PCB, } \\
\text { acetaminophen, aflatoxin B1, ammonium chloride, } \\
\text { bisphenol A, dibutyl phthalate, diethylnitrosamine, } \\
\text { methoxychlor, tobacco smoke pollution }\end{array}$ & $\begin{array}{l}23974009,21802500,20382639, \\
21592394,22298810,19114083, \\
17562736,19770486,16483693, \\
23359474,23359474,15833021, \\
23303685,18087596\end{array}$ \\
\hline VWAЗA & rs61744122 & copper sulfate, tetrachlorodibenzodioxin & 19549813,21570461 \\
\hline$R S U 1$ & rs4748309 & $\begin{array}{c}\text { arsenite, estradiol, PCB, aflatoxin B1, benzo(a)pyrene, } \\
\text { tetrachlorodibenzodioxin }\end{array}$ & $\begin{array}{l}23974009,19167446,19114083 \text {, } \\
19770486,21715664,21570461\end{array}$ \\
\hline ACOT11 & rs 1755588 & $\begin{array}{l}\text { aflatoxin B1, atrazine, benzo(a)pyrene, copper sulfate, } \\
\text { fonofos, hydrogen peroxide, parathion, terbufos, } \\
\text { testosterone }\end{array}$ & $\begin{array}{l}21641981,22378314,22316170, \\
19549813,22847954,20044591, \\
22847954,22847954,21592394\end{array}$ \\
\hline OLFM3 & rs $10493958^{*}$ & atrazine, benzo(a)pyrene, acetaminophen & $22378314,22316170,17562736$ \\
\hline NEDD4 & rs $7169431 *$ & $\begin{array}{c}\text { acetaminophen, arsenite, copper sulfate, potassium } \\
\text { chromate(VI), benzo(a)pyrene, diuron, ethinyl estradiol, } \\
\text { testosterone, tetrachlorodibenzodioxin }\end{array}$ & $\begin{array}{l}21420995,23974009,19549813, \\
22079256,20127859,25152437, \\
17555576,21669218,21570461\end{array}$ \\
\hline$R F X 7$ & rs $7169431^{*}$ & atrazine, estradiol, copper sulfate, formaldehyde & $\begin{array}{c}22378314,22574217,19549813, \\
20655997\end{array}$ \\
\hline BBS9 & rs 17170286 & $\begin{array}{l}\text { aflatoxin B1, air pollutants, arsenic trioxide, benzo(a)pyrene, } \\
\text { cobaltous chloride, formaldehyde, tetrachlorodibenzodioxin }\end{array}$ & $\begin{array}{c}20106945,21757418,20458559, \\
21632981,19320972,23649840, \\
21570461\end{array}$ \\
\hline BST1 & rs11945730* & $\begin{array}{l}\text { aflatoxin B1, benzo(a)pyrene diol epoxide, cobaltous } \\
\text { chloride, tetrachlorodibenzodioxin, acetaminophen, arsenic, } \\
\text { chlorpyrifos, cocaine, diazinon, ethanol, testosterone, } \\
\text { tobacco smoke pollution }\end{array}$ & $\begin{array}{l}22100608,20382639,19320972, \\
17101203,17562736,19654921, \\
21968025,20187946,21968025, \\
15353170,15788153,20133372\end{array}$ \\
\hline
\end{tabular}


Appendix Table 20 continued....

\begin{tabular}{|c|c|c|c|}
\hline Gene/region & SNP & Chemical interaction & PUBMED ID \\
\hline & & arsenic trioxide, parathion, progesterone, terbufos, arsenic, & $18297255,22847954,21768398$, \\
estradiol, paraquat, particulate matter, progesterone, & $22847954,19654921,21840325$, \\
testosterone, tetrachlorodibenzodioxin & $18198484,23638009,21770760$, \\
& rs11945730* & acetaminophen, aflatoxin B1, benzo(a)pyrene, copper & 21840325,21570461 \\
\hline & & sulfate, potassium chromate(VI), PCB, & $19549813,22079256,19114083$, \\
tetrachlorodibenzodioxin & 24680724 \\
\hline
\end{tabular}

$\mathrm{SNP}=$ single nucleotide polymorphism 
Appendix Table 21: Deleterious effect of missense variations associated with gender-specificity in childhood ALL risk

\begin{tabular}{|c|c|c|c|c|c|c|}
\hline $\begin{array}{c}\text { SNP } \\
\text { (missense variations) }\end{array}$ & $\begin{array}{c}\text { Gender- } \\
\text { specific results }\end{array}$ & $\begin{array}{l}\text { SIFT } \\
\text { score }\end{array}$ & $\begin{array}{c}\text { SIFT } \\
\text { prediction }\end{array}$ & $\begin{array}{c}\text { SIFT } \\
\text { confidence }\end{array}$ & $\begin{array}{l}\text { PolyPhen } \\
\text { score }\end{array}$ & $\begin{array}{l}\text { PolyPhen } \\
\text { prediction }\end{array}$ \\
\hline rs9913430 & Gender $\mathrm{P}$ value & 0.74 & tolerated & high & 0 & benign \\
\hline rs61748746 & Gender $\mathrm{P}$ value & 0.25 & tolerated & high & 0.24 & possibly damaging \\
\hline rs61748749 & Gender $\mathrm{P}$ value & 0.43 & tolerated & high & 0.24 & possibly damaging \\
\hline rs35693261 & Gender OR & 0.01 & damaging & high & 0.01 & benign \\
\hline rs4770684 & Gender OR & 0.63 & tolerated & high & 0.03 & benign \\
\hline rs35665085 & Gender OR & 0.13 & tolerated & high & 0.01 & benign \\
\hline rs3847262 & Gender OR & 0.05 & damaging & high & 0.93 & probably damaging \\
\hline rs17080410 & Gender OR & 1 & tolerated & low & 0 & benign \\
\hline rs61742396 & Gender OR & 1 & tolerated & low & 0 & benign \\
\hline rs35672330 & Gender OR & 0 & damaging & high & 1 & probably damaging \\
\hline rs11751765 & Gender OR & 0.16 & tolerated & high & 0.91 & probably damaging \\
\hline rs73022563 & Gender OR & 0.05 & damaging & high & 0.96 & probably damaging \\
\hline rs61731389 & Gender OR & 0.06 & tolerated & high & 0.86 & probably damaging \\
\hline rs143021649 & Gender OR & 0.29 & tolerated & high & 0.01 & benign \\
\hline rs61745456 & Gender OR & 0.97 & tolerated & high & 0.98 & probably damaging \\
\hline rs36041430 & Gender OR & 0.22 & tolerated & high & 0.001 & benign \\
\hline rs7829965 & Gender OR & 0.72 & tolerated & high & 0 & benign \\
\hline rs34022679 & Gender OR & 0 & damaging & high & 1 & probably damaging \\
\hline rs2293624 & Gender OR & 0.11 & tolerated & low & 0.003 & benign \\
\hline rs62640905 & Gender OR & 0.36 & tolerated & high & 0.52 & possibly damaging \\
\hline rs16973890 & Gender OR & 0.08 & tolerated & high & 0.52 & possibly damaging \\
\hline
\end{tabular}




Appendix Table 21 continued...
\begin{tabular}{|c|c|c|c|c|c|c|}
\hline $\begin{array}{c}\text { SNP } \\
\text { (missense variations) }\end{array}$ & $\begin{array}{c}\text { Gender- } \\
\text { specific results }\end{array}$ & $\begin{array}{c}\text { SIFT } \\
\text { score }\end{array}$ & $\begin{array}{c}\text { SIFT } \\
\text { prediction }\end{array}$ & $\begin{array}{c}\text { SIFT } \\
\text { confidence }\end{array}$ & $\begin{array}{c}\text { PolyPhen } \\
\text { score }\end{array}$ & $\begin{array}{c}\text { PolyPhen } \\
\text { prediction }\end{array}$ \\
\hline rs117923468 & Gender OR & 0.5 & tolerated & high & 0.21 & possibly damaging \\
\hline rs182319278 & Gender OR & 0.97 & tolerated & low & 0 & benign \\
\hline rs4645656 & Gender OR & 0.04 & tolerated & high & 0.56 & possibly damaging \\
\hline rs12722039 & Gender OR & 0.5 & tolerated & low & 0 & benign \\
\hline rs12722042 & Gender OR & 0.66 & tolerated & low & 0.36 & possibly damaging \\
\hline rs130069 & Gender OR & 0.47 & tolerated & high & 0.24 & possibly damaging \\
\hline rs78888579 & Gender OR & 0.51 & tolerated & high & 0 & benign \\
\hline rs56278097 & Gender OR & 0.22 & tolerated & high & 0 & benign \\
\hline rs2229528 & Gender OR & 0.05 & tolerated & high & 0.48 & possibly damaging \\
\hline rs 55882716 & Gender OR & 0.43 & tolerated & low & 0 & benign \\
\hline rs34664882 & Gender OR & 0.02 & damaging & high & 0.003 & benign \\
\hline rs80040922 & Gender OR & 0.02 & damaging & high & 0.96 & probably damaging \\
\hline rs61741435 & Gender OR & 0.71 & tolerated & high & 0.91 & probably damaging \\
\hline rs147596194 & Gender OR & 0 & damaging & low & 0 & benign \\
\hline
\end{tabular}

SNP = single nucleotide polymorphisms; SIFT \& PolyPhen: databases for missense SNP prediction 
Appendix Table 22: Deleterious effect of missense variations associated with age-specificity in childhood ALL risk

\begin{tabular}{|c|c|c|c|c|c|c|}
\hline $\begin{array}{c}\text { SNP } \\
\text { (missense variations) }\end{array}$ & $\begin{array}{l}\text { Age-specific } \\
\text { results }\end{array}$ & $\begin{array}{l}\text { SIFT } \\
\text { score }\end{array}$ & $\begin{array}{c}\text { SIFT } \\
\text { prediction }\end{array}$ & $\begin{array}{c}\text { SIFT } \\
\text { confidence }\end{array}$ & $\begin{array}{l}\text { PolyPhe } \\
\text { score }\end{array}$ & PolyPhen prediction \\
\hline rs2274068 & Age $P$ value & 0.45 & Tolerated & High & 0 & Benign \\
\hline rs 762562 & Age $P$ value & 1 & Tolerated & High & 0 & Benign \\
\hline rs735482 & Age $\mathrm{P}$ value & 0.56 & Tolerated & High & 0 & Benign \\
\hline rs9897794 & Age $P$ value & 0.34 & Tolerated & High & 0.003 & Benign \\
\hline rs2101919 & Age $\mathrm{P}$ value & 0.57 & Tolerated & High & 0 & Benign \\
\hline rs6972158 & Age $P$ value & 0.43 & Tolerated & High & 0 & Benign \\
\hline rs56291145 & Age OR & 0.16 & Tolerated & Low & 0.02 & Benign \\
\hline rs35384751 & Age OR & 0 & Damaging & High & 0.63 & Possibly damaging \\
\hline rs10999147 & Age OR & 0.4 & Tolerated & High & 0.24 & Possibly damaging \\
\hline rs17409162 & Age OR & 0.22 & Tolerated & High & 0.97 & Probably damaging \\
\hline rs16916188 & Age OR & 0.09 & Tolerated & High & 0.14 & Benign \\
\hline rs35537868 & Age OR & 0.82 & Tolerated & High & 0.08 & Benign \\
\hline rs35340237 & Age OR & 0.84 & Tolerated & High & 0 & Benign \\
\hline rs17407084 & Age OR & 0 & Damaging & High & 1 & Probably damaging \\
\hline rs41309927 & Age OR & 0.34 & Tolerated & High & 0 & Benign \\
\hline rs36086854 & Age OR & 0.34 & Tolerated & High & 0.05 & Benign \\
\hline rs34139105 & Age OR & 0.29 & Tolerated & High & 0.4 & Possibly damaging \\
\hline rs36068434 & Age OR & 0.02 & Tolerated & Low & 0 & Benign \\
\hline rs11651675 & Age OR & 0.62 & Tolerated & High & 0.001 & Benign \\
\hline rs41279492 & effect size & 0.35 & Tolerated & High & 0 & Benign \\
\hline
\end{tabular}




Appendix Table 22 continued...
\begin{tabular}{|c|c|c|c|c|c|c|}
\hline $\begin{array}{c}\text { SNP } \\
\text { (missense variations) }\end{array}$ & $\begin{array}{c}\text { Age-specific } \\
\text { results }\end{array}$ & $\begin{array}{c}\text { SIFT } \\
\text { score }\end{array}$ & $\begin{array}{c}\text { SIFT } \\
\text { prediction }\end{array}$ & $\begin{array}{c}\text { SIFT } \\
\text { confidence }\end{array}$ & $\begin{array}{c}\text { PolyPhe } \\
\text { score }\end{array}$ & PolyPhen prediction \\
\hline rs 141872266 & Age OR & 0.01 & Damaging & High & 0.04 & Benign \\
\hline rs138700801 & Age OR & 0.19 & Tolerated & High & 0.08 & Benign \\
\hline rs9728577 & Age OR & 0.05 & Damaging & High & 0.006 & Benign \\
\hline rs3204798 & Age OR & 0.11 & Tolerated & High & 0 & Benign \\
\hline rs41285557 & Age OR & 0.68 & Tolerated & High & 0.001 & Benign \\
\hline rs35424709 & Age OR & 0.39 & Tolerated & High & 1 & Probably damaging \\
\hline rs61744122 & Age OR & 0.33 & Tolerated & High & 0.16 & Benign \\
\hline rs78323957 & Age OR & 0.13 & Tolerated & High & 0.001 & Benign \\
\hline rs35384751 & Age OR & 0.02 & Damaging & High & 0.63 & Possibly damaging \\
\hline rs35340237 & Age OR & 1 & Tolerated & High & 0 & Benign \\
\hline
\end{tabular}

SNP = single nucleotide polymorphisms; SIFT \& PolyPhen: databases for missense SNP prediction 
Appendix Table 23: Time-to-event analysis of identified gender- and age-specific childhood ALL risk markers

\begin{tabular}{|c|c|c|c|c|c|c|c|}
\hline & & Overall & & Male & & Female & \\
\hline SNP & $\begin{array}{c}\text { Log rank } \\
\text { P value }\end{array}$ & HR (95\% CI) & $P$ value & HR (95\% CI) & $P$ value & HR (95\% CI) & $P$ value \\
\hline rs10505918 ${ }^{(\mathrm{D})^{*}}$ & $<1 \times 10^{-4}$ & $1.69(1.27-2.24)$ & $<1 \times 10^{-3}$ & $1.55(1.06-2.25)$ & 0.02 & $1.93(1.25-2.96)$ & $3 \times 10^{-3}$ \\
\hline rs2949827 $7^{(\mathrm{D})^{*}}$ & $<1 \times 10^{-4}$ & $0.54(0.38-0.77)$ & $1 \times 10^{-3}$ & $0.72(0.46-1.12)$ & 0.15 & $0.39(0.23-0.66)$ & $<1 \times 10^{-3}$ \\
\hline rs2212824 ${ }^{(\mathrm{D})^{*}}$ & $<1 \times 10^{-4}$ & $1.76(1.30-2.38)$ & $<1 \times 10^{-3}$ & $1.76(1.20-2.59)$ & $4 \times 10^{-3}$ & $1.76(1.09-2.83)$ & 0.02 \\
\hline rs1209145 ${ }^{(\mathrm{D})^{*}}$ & $<1 \times 10^{-4}$ & $1.80(1.31-2.48)$ & $<1 \times 10^{-3}$ & $1.62(1.05-2.51)$ & 0.03 & $2.05(1.28-3.27)$ & $3 \times 10^{-3}$ \\
\hline rs1209145 ${ }^{(\mathrm{D})^{*}}$ & $<1 \times 10^{-4}$ & $1.76(1.28-2.41)$ & $<1 \times 10^{-3}$ & $1.62(1.05-2.51)$ & 0.03 & $2.05(1.28-3.27)$ & $3 \times 10^{-3}$ \\
\hline rs $738027^{(\mathrm{D})^{*}}$ & $<1 \times 10^{-4}$ & $1.62(1.22-2.15)$ & $1 \times 10^{-3}$ & $1.62(1.10-2.38)$ & 0.01 & $1.62(1.06-2.47)$ & 0.03 \\
\hline rs2101919 ${ }^{(\mathrm{D})^{*}}$ & $<1 \times 10^{-4}$ & $0.61(0.46-0.80)$ & $1 \times 10^{-3}$ & $0.64(0.44-0.94)$ & 0.02 & $0.56(0.37-0.86)$ & $8 \times 10^{-3}$ \\
\hline rs9809647 ${ }^{(\mathrm{D})^{*}}$ & $<1 \times 10^{-4}$ & $0.60(0.45-0.78)$ & $1 \times 10^{-3}$ & $0.57(0.39-0.84)$ & $4 \times 10^{-3}$ & $0.64(0.42-0.98)$ & 0.04 \\
\hline rs $10133677^{(\mathrm{D})^{*}}$ & $<1 \times 10^{-4}$ & $1.78(1.28-2.46)$ & $1 \times 10^{-3}$ & $1.65(1.05-2.61)$ & 0.03 & $1.92(1.21-3.06)$ & $6 \times 10^{-3}$ \\
\hline rs10999147 ${ }^{(\mathrm{D})^{* *}}$ & $<1 \times 10^{-4}$ & $2.55(1.59-4.08)$ & $<1 \times 10^{-3}$ & $2.01(1.09-3.71)$ & 0.03 & $3.90(1.85-8.22)$ & $<1 \times 10^{-3}$ \\
\hline rs1882591 ${ }^{(\mathrm{D})^{*}}$ & $1 \times 10^{-4}$ & $0.63(0.48-0.83)$ & $1 \times 10^{-3}$ & $0.55(0.38-0.81)$ & $2 \times 10^{-3}$ & $0.75(0.49-1.13)$ & 0.17 \\
\hline rs $10099957^{(\mathrm{D})^{* *}}$ & $1 \times 10^{-4}$ & $2.00(1.35-2.95$ & $<1 \times 10^{-3}$ & $2.32(1.36-3.95)$ & $2 \times 10^{-3}$ & $1.70(0.95-3.02)$ & 0.07 \\
\hline rs4741086 $6^{(\mathrm{D})^{* *}}$ & $1 \times 10^{-4}$ & $1.75(1.26-2.44)$ & $1 \times 10^{-3}$ & $1.48(0.98-2.24)$ & 0.06 & $2.39(1.40-4.08)$ & $1 \times 10^{-3}$ \\
\hline rs7091901 ${ }^{(\mathrm{D})^{* *}}$ & $1 \times 10^{-4}$ & $2.00(1.33-3.01)$ & $1 \times 10^{-3}$ & $1.76(1.01-3.06)$ & 0.05 & $2.38(1.30-4.35)$ & $5 \times 10^{-3}$ \\
\hline rs $1457759^{(\mathrm{D})^{* *}}$ & $1 \times 10^{-4}$ & $2.05(1.37-3.06)$ & $<1 \times 10^{-3}$ & $2.43(1.43-4.16)$ & $1 \times 10^{-3}$ & $1.67(0.90-3.09)$ & 0.10 \\
\hline rs $10826912^{(\mathrm{D})^{* *}}$ & $1 \times 10^{-4}$ & $2.00(1.32-3.02)$ & $1 \times 10^{-3}$ & $1.74(0.98-3.06)$ & 0.06 & $2.38(1.30-4.35)$ & $5 \times 10^{-3}$ \\
\hline rs $237320^{(\mathrm{D})^{*}}$ & $2 \times 10^{-4}$ & $0.62(0.45-0.84)$ & $2 \times 10^{-3}$ & $0.65(0.42-1.00)$ & 0.05 & $0.58(0.38-0.91)$ & 0.02 \\
\hline rs17109582 ${ }^{(\mathrm{D})^{* *}}$ & $2 \times 10^{-4}$ & $2.05(1.33-3.16)$ & $1 \times 10^{-3}$ & $2.13(1.21-3.74)$ & $9 \times 10^{-3}$ & $1.96(1.00-3.82)$ & 0.05 \\
\hline rs9856456 $6^{(\mathrm{D})^{* *}}$ & $2 \times 10^{-4}$ & $2.27(1.37-3.75)$ & $1 \times 10^{-3}$ & $1.89(1.05-3.40)$ & 0.03 & $4.36(1.68-11.32)$ & $2 \times 10^{-3}$ \\
\hline rs1894381 ${ }^{(\mathrm{D})^{*}}$ & $3 \times 10^{-4}$ & $0.63(0.47-0.85)$ & $2 \times 10^{-3}$ & $0.54(0.36-0.80)$ & $2 \times 10^{-3}$ & $0.78(0.51-1.20)$ & 0.26 \\
\hline rs $10079494^{(\mathrm{D})^{*}}$ & $4 \times 10^{-4}$ & $1.53(1.16-2.03)$ & $3 \times 10^{-3}$ & $1.44(0.99-2.10)$ & 0.06 & $1.69(1.11-2.59)$ & 0.02 \\
\hline
\end{tabular}


Appendix Table 23 continued....

\begin{tabular}{|c|c|c|c|c|c|c|c|}
\hline & & Overall & & Male & & Female & \\
\hline SNP & $\begin{array}{c}\text { Log rank } \\
\text { P value }\end{array}$ & HR (95\% CI) & $P$ value & HR (95\% CI) & $P$ value & HR (95\% CI) & $P$ value \\
\hline rs35424709 $9^{(\mathrm{D})^{* *}}$ & $4 \times 10^{-4}$ & $1.83(1.24-2.68)$ & $2 \times 10^{-3}$ & $1.79(0.98-3.28)$ & 0.06 & $1.85(1.13-3.05)$ & 0.02 \\
\hline rs2388773 ${ }^{(\mathrm{D})^{*}}$ & $5 \times 10^{-4}$ & $0.65(0.49-0.87)$ & $4 \times 10^{-3}$ & $0.58(0.39-0.86)$ & $6 \times 10^{-3}$ & $0.77(0.51-1.17)$ & 0.22 \\
\hline rs9694342 $2^{(\mathrm{D})^{*}}$ & $6 \times 10^{-4}$ & $0.66(0.50-0.87)$ & $3 \times 10^{-3}$ & $0.63(0.43-0.92)$ & 0.02 & $0.69(0.45-1.04)$ & 0.08 \\
\hline rs $16827743^{(\mathrm{D})^{* *}}$ & $6 \times 10^{-4}$ & $2.00(1.27-3.14)$ & $3 \times 10^{-3}$ & $1.81(1.02-3.20)$ & 0.04 & $2.40(1.14-5.06)$ & 0.02 \\
\hline rs8011343 ${ }^{(\mathrm{D})^{*}}$ & $7 \times 10^{-4}$ & $1.57(1.16-2.13)$ & $4 \times 10^{-3}$ & $1.78(1.16-2.74)$ & $9 \times 10^{-3}$ & $1.40(0.90-2.17)$ & 0.13 \\
\hline rs1493897 ${ }^{(\mathrm{D})^{*}}$ & $7 \times 10^{-4}$ & $0.67(0.50-0.88)$ & $5 \times 10^{-3}$ & $0.59(0.40-0.86)$ & $6 \times 10^{-3}$ & $0.79(0.52-1.21)$ & 0.27 \\
\hline rs1915164 $4^{(\mathrm{D})^{* *}}$ & $7 \times 10^{-4}$ & $2.00(1.26-3.19)$ & $3 \times 10^{-3}$ & $1.75(0.84-3.66)$ & 0.13 & $2.21(1.21-4.03)$ & 0.01 \\
\hline rs11014598 ${ }^{(\mathrm{D}) \dagger \dagger}$ & $8 \times 10^{-4}$ & $0.45(0.25-0.81)$ & $8 \times 10^{-3}$ & $0.47(0.24-0.90)$ & 0.02 & $0.38(0.09-1.54)$ & 0.18 \\
\hline rs6972158 ${ }^{(\mathrm{D})^{*}}$ & $8 \times 10^{-4}$ & $1.50(1.14-1.98)$ & $4 \times 10^{-3}$ & $1.59(1.09-2.31)$ & 0.02 & $1.39(0.92-2.10)$ & 0.12 \\
\hline rs7320982 ${ }^{(\mathrm{D})^{*}}$ & $8 \times 10^{-4}$ & $0.66(0.50-0.88)$ & $5 \times 10^{-3}$ & $0.85(0.59-1.23)$ & 0.39 & $0.48(0.31-0.74)$ & $1 \times 10^{-3}$ \\
\hline rs6492498 ${ }^{(\mathrm{D})^{*}}$ & $8 \times 10^{-4}$ & $0.66(0.50-0.88)$ & $4 \times 10^{-3}$ & $0.61(0.42-0.89)$ & 0.01 & $0.74(0.49-1.13)$ & 0.16 \\
\hline rs $10250709^{(\mathrm{D})^{*}}$ & $8 \times 10^{-4}$ & $1.50(1.14-1.98)$ & $4 \times 10^{-3}$ & $1.59(1.09-2.31)$ & 0.02 & $1.39(0.92-2.10)$ & 0.12 \\
\hline rs1191818 ${ }^{(\mathrm{D})^{*}}$ & $8 \times 10^{-4}$ & $0.65(0.48-0.87)$ & $4 \times 10^{-3}$ & $0.65(0.44-0.96)$ & 0.03 & $0.66(0.41-1.05)$ & 0.08 \\
\hline rs2909644 ${ }^{(\mathrm{D})^{* *}}$ & $8 \times 10^{-4}$ & $2.10(1.26-3.48)$ & $4 \times 10^{-3}$ & $1.70(0.89-3.27)$ & 0.11 & $3.14(1.39-7.11)$ & $6 \times 10^{-3}$ \\
\hline rs6708453 $3^{(\mathrm{D})^{* *}}$ & $8 \times 10^{-4}$ & $1.61(1.17-2.22)$ & $4 \times 10^{-3}$ & $1.86(1.23-2.83)$ & $4 \times 10^{-3}$ & $1.31(0.78-2.20)$ & 0.31 \\
\hline rs $222314^{(\mathrm{D})^{*}}$ & $8 \times 10^{-4}$ & $1.56(1.15-2.13)$ & $4 \times 10^{-3}$ & $1.47(0.98-2.20)$ & 0.06 & $1.72(1.06-2.78)$ & 0.03 \\
\hline rs139886 ${ }^{(\mathrm{D})^{*}}$ & $8 \times 10^{-4}$ & $1.51(1.13-2.02)$ & $5 \times 10^{-3}$ & $1.32(0.90-1.93)$ & 0.16 & $1.84(1.16-2.81)$ & $9 \times 10^{-3}$ \\
\hline rs $17409162^{(\mathrm{D})^{* *}}$ & $8 \times 10^{-4}$ & $2.09(1.27-3.44)$ & $4 \times 10^{-3}$ & $2.06(1.03-4.11)$ & 0.04 & $2.13(1.04-4.33)$ & 0.04 \\
\hline rs9560160 ${ }^{(\mathrm{D})^{* *}}$ & $8 \times 10^{-4}$ & $1.73(1.19-2.52)$ & $4 \times 10^{-3}$ & $1.35(0.81-2.23)$ & 0.25 & $2.50(1.43-4.36)$ & $1 \times 10^{-3}$ \\
\hline rs7791037 ${ }^{(\mathrm{R}) \dagger}$ & $1 \times 10^{-3}$ & $1.66(1.17-2.37)$ & $4 \times 10^{-3}$ & $1.78(1.04-3.06)$ & 0.04 & $1.59(1.00-2.51)$ & 0.05 \\
\hline rs1881116 ${ }^{(\mathrm{D})^{*}}$ & $1 \times 10^{-3}$ & $1.48(1.12-1.95)$ & $6 \times 10^{-3}$ & $1.17(0.81-1.68)$ & 0.41 & $2.29(1.46-3.60)$ & $<1 \times 10^{-3}$ \\
\hline
\end{tabular}


Appendix Table 23 continued....

\begin{tabular}{|c|c|c|c|c|c|c|c|}
\hline & & Overall & & Male & & Female & \\
\hline SNP & $\begin{array}{c}\text { Log rank } \\
\text { P value }\end{array}$ & HR (95\% CI) & $P$ value & HR (95\% CI) & $P$ value & HR (95\% CI) & P value \\
\hline rs11997355 (D)* & $1 \times 10^{-3}$ & $0.61(0.43-0.87)$ & $7 \times 10^{-3}$ & $0.62(0.38-0.98)$ & 0.04 & $0.61(0.36-1.04)$ & 0.07 \\
\hline rs7720650 ${ }^{(\mathrm{D})^{*}}$ & $1 \times 10^{-3}$ & $0.65(0.48-0.88)$ & $6 \times 10^{-3}$ & $0.76(0.50-1.16)$ & 0.20 & $0.55(0.35-0.85)$ & $7 \times 10^{-3}$ \\
\hline rs2396653 ${ }^{(\mathrm{D})^{*}}$ & $1 \times 10^{-3}$ & $0.66(0.50-0.89)$ & $6 \times 10^{-3}$ & $0.82(0.55-1.20)$ & 0.31 & $0.52(0.33-0.80)$ & $3 \times 10^{-3}$ \\
\hline rs1151277 ${ }^{(\mathrm{D})^{*}}$ & $1 \times 10^{-3}$ & $0.66(0.49-0.89)$ & $7 \times 10^{-3}$ & $0.55(0.37-0.82)$ & $4 \times 10^{-3}$ & $0.84(0.54-1.31)$ & 0.44 \\
\hline rs12073211 $1^{(\mathrm{D})^{*}}$ & $1 \times 10^{-3}$ & $0.67(0.50-0.90)$ & $7 \times 10^{-3}$ & $0.75(0.51-1.10)$ & 0.14 & $0.58(0.37-0.89)$ & 0.01 \\
\hline rs3825594 ${ }^{(\mathrm{D})^{*}}$ & $1 \times 10^{-3}$ & $0.67(0.51-0.89)$ & $5 \times 10^{-3}$ & $0.75(0.51-1.08)$ & 0.12 & $0.59(0.39-0.89)$ & 0.01 \\
\hline rs1610315 ${ }^{(\mathrm{D})^{*}}$ & $1 \times 10^{-3}$ & $0.68(0.51-0.89)$ & $6 \times 10^{-3}$ & $0.57(0.39-0.84)$ & $4 \times 10^{-3}$ & $0.83(0.55-1.24)$ & 0.36 \\
\hline rs9897794 ${ }^{(\mathrm{D})^{*}}$ & $1 \times 10^{-3}$ & $0.66(0.49-0.89)$ & $6 \times 10^{-3}$ & $0.76(0.52-1.12)$ & 0.17 & $0.49(0.31-0.80)$ & $4 \times 10^{-3}$ \\
\hline rs8014810 ${ }^{(\mathrm{D})^{*}}$ & $1 \times 10^{-3}$ & $0.67(0.50-0.90)$ & $7 \times 10^{-3}$ & $0.69(0.46-1.02)$ & 0.06 & $0.64(0.41-0.98)$ & 0.04 \\
\hline rs3954950 ${ }^{(\mathrm{D})^{*}}$ & $1 \times 10^{-3}$ & $0.68(0.51-0.89)$ & $6 \times 10^{-3}$ & $0.67(0.46-0.98)$ & 0.04 & $0.67(0.44-1.02)$ & 0.06 \\
\hline rs56291145 ${ }^{(\mathrm{D})^{* *}}$ & $1 \times 10^{-3}$ & $2.06(1.24-3.43)$ & $5 \times 10^{-3}$ & $1.90(0.98-3.68)$ & 0.06 & $2.34(1.06-5.20)$ & 0.04 \\
\hline rs9861022 $2^{(\mathrm{D})^{* *}}$ & $1 \times 10^{-3}$ & $1.94(1.23-3.05)$ & $4 \times 10^{-3}$ & $1.73(0.98-3.07)$ & 0.06 & $2.40(1.14-5.06)$ & 0.02 \\
\hline rs $10493958^{(\mathrm{D})^{* *}}$ & $1 \times 10^{-3}$ & $1.99(1.23-3.21)$ & $5 \times 10^{-3}$ & $1.95(0.98-3.87)$ & 0.06 & $2.03(1.04-3.97)$ & 0.04 \\
\hline rs9392857 ${ }^{(\mathrm{D})^{*}}$ & $2 \times 10^{-3}$ & $0.68(0.51-0.90)$ & $8 \times 10^{-3}$ & $0.77(0.52-1.13)$ & 0.18 & $0.55(0.36-0.85)$ & $8 \times 10^{-3}$ \\
\hline rs4948088 ${ }^{(\mathrm{D})^{*}}$ & $2 \times 10^{-3}$ & $0.61(0.42-0.90)$ & $8 \times 10^{-3}$ & $0.72(0.43-1.21)$ & 0.21 & $0.49(0.28-0.86)$ & 0.01 \\
\hline rs7583258 $8^{(\mathrm{D})^{*}}$ & $2 \times 10^{-3}$ & $0.66(0.48-0.91)$ & 0.01 & $0.55(0.36-0.84)$ & $5 \times 10^{-3}$ & $0.85(0.53-1.35)$ & 0.49 \\
\hline rs7722067 ${ }^{(\mathrm{D})^{*}}$ & $2 \times 10^{-3}$ & $0.68(0.51-0.91)$ & $9 \times 10^{-3}$ & $0.74(0.51-1.08)$ & 0.12 & $0.61(0.40-0.94)$ & 0.03 \\
\hline rs2065212 ${ }^{(\mathrm{D})^{*}}$ & $2 \times 10^{-3}$ & $0.68(0.52-0.91)$ & $8 \times 10^{-3}$ & $0.64(0.44-0.93)$ & 0.02 & $0.74(0.49-1.13)$ & 0.17 \\
\hline rs2830698 ${ }^{(\mathrm{R})^{*}}$ & $2 \times 10^{-3}$ & $0.51(0.30-0.87)$ & 0.01 & $0.53(0.25-1.11)$ & 0.09 & $0.49(0.22-1.07)$ & 0.08 \\
\hline rs4748309 $9^{(\mathrm{D})^{* *}}$ & $2 \times 10^{-3}$ & $1.78(1.18-2.65)$ & $6 \times 10^{-3}$ & $1.51(0.87-2.62)$ & 0.14 & $2.18(1.20-3.98)$ & 0.01 \\
\hline rs $11945730^{(\mathrm{D})^{* *}}$ & $2 \times 10^{-3}$ & $1.66(1.15-2.41)$ & $7 \times 10^{-3}$ & $1.42(0.88-2.31)$ & 0.16 & $2.13(1.20-3.81)$ & 0.01 \\
\hline rs7294845 ${ }^{(\mathrm{D}) \dagger}$ & $3 \times 10^{-3}$ & $1.43(1.08-1.89)$ & 0.01 & $1.32(0.90-1.93)$ & 0.15 & $1.57(1.04-2.38)$ & 0.03 \\
\hline
\end{tabular}


Appendix Table 23 continued....

\begin{tabular}{|c|c|c|c|c|c|c|c|}
\hline & & Overall & & Male & & Female & \\
\hline SNP & $\begin{array}{c}\text { Log rank } \\
\text { P value }\end{array}$ & HR $(95 \%$ CI) & $P$ value & HR $(95 \%$ CI) & $P$ value & HR $(95 \%$ CI) & $P$ value \\
\hline rs6739633 ${ }^{(\mathrm{D})^{*}}$ & $3 \times 10^{-3}$ & $1.44(1.08-1.91)$ & 0.01 & $1.23(0.83-1.81)$ & 0.30 & $1.85(1.18-2.88)$ & $7 \times 10^{-3}$ \\
\hline rs $139866^{(\mathrm{D})^{*}}$ & $3 \times 10^{-3}$ & $1.44(1.08-1.92)$ & 0.01 & $1.27(0.87-1.86)$ & 0.22 & $1.73(1.11-2.69)$ & 0.02 \\
\hline rs3800292 $2^{(\mathrm{D})^{*}}$ & $3 \times 10^{-3}$ & $0.55(0.34-0.88)$ & 0.02 & $0.58(0.25-1.32)$ & 0.19 & $0.54(0.30-0.96)$ & 0.04 \\
\hline rs36068434 $4^{(\mathrm{D})^{* *}}$ & $3 \times 10^{-3}$ & $1.93(1.17-3.20)$ & 0.01 & $1.96(1.05-3.69)$ & 0.04 & $1.88(0.81-4.38)$ & 0.14 \\
\hline rs16909509 & $3 \times 10^{-3}$ & $1.86(1.10-3.13)$ & 0.02 & $2.17(1.03-4.55)$ & 0.04 & $1.62(0.78-3.37)$ & 0.20 \\
\hline rs $12705300^{(\mathrm{D})^{* *}}$ & $3 \times 10^{-3}$ & $1.64(1.12-2.40)$ & 0.01 & $1.83(1.10-3.07)$ & 0.02 & $1.44(0.81-2.56)$ & 0.21 \\
\hline rs $4821717^{(\mathrm{D})^{*}}$ & $4 \times 10^{-3}$ & $1.42(1.07-1.88)$ & 0.02 & $1.35(0.92-1.96)$ & 0.12 & $1.51(0.98-2.33)$ & 0.06 \\
\hline rs7157104 $4^{(\mathrm{D})^{*}}$ & $4 \times 10^{-3}$ & $0.71(0.53-0.94)$ & 0.02 & $0.76(0.52-1.12)$ & 0.12 & $0.64(0.42-0.98)$ & 0.04 \\
\hline rs973358 $8^{(\mathrm{D})^{*}}$ & $4 \times 10^{-3}$ & $1.44(1.07-1.92)$ & 0.02 & $1.66(1.12-2.46)$ & 0.01 & $1.18(0.76-1.83)$ & 0.46 \\
\hline rs41309927 $7^{(\mathrm{D})^{* *}}$ & $4 \times 10^{-3}$ & $1.85(1.15-2.99)$ & 0.01 & $1.52(0.84-2.74)$ & 0.17 & $2.93(1.32-6.52)$ & $8 \times 10^{-3}$ \\
\hline rs276725 $5^{(\mathrm{D})^{* *}}$ & $4 \times 10^{-3}$ & $1.75(1.14-2.71)$ & 0.01 & $2.28(1.26-4.15)$ & $7 \times 10^{-3}$ & $1.37(0.72-2.58)$ & 0.33 \\
\hline rs11250832 $2^{(\mathrm{D})^{* *}}$ & $4 \times 10^{-3}$ & $1.64(1.11-2.42)$ & 0.01 & $1.54(0.97-2.46)$ & 0.07 & $1.89(0.94-3.80)$ & 0.08 \\
\hline rs2899293 $3^{(\mathrm{D})^{*}}$ & $5 \times 10^{-3}$ & $1.43(1.06-1.93)$ & 0.02 & $1.26(0.86-1.86)$ & 0.24 & $1.76(1.10-2.83)$ & 0.02 \\
\hline rs7318021 $1^{(\mathrm{D})^{*}}$ & $5 \times 10^{-3}$ & $0.72(0.55-0.94)$ & 0.02 & $0.61(0.42-0.89)$ & 0.01 & $0.86(0.57-1.29)$ & 0.46 \\
\hline rs8021355 $5^{(\mathrm{D})^{*}}$ & $5 \times 10^{-3}$ & $0.71(0.53-0.94)$ & 0.02 & $0.77(0.53-1.13)$ & 0.19 & $0.63(0.41-0.96)$ & 0.02 \\
\hline rs7144565 $5^{(\mathrm{D})^{*}}$ & $5 \times 10^{-3}$ & $0.71(0.53-0.94)$ & 0.02 & $0.77(0.53-1.13)$ & 0.19 & $0.63(0.41-0.96)$ & 0.02 \\
\hline rs2274068 $8^{(\mathrm{D})^{*}}$ & $5 \times 10^{-3}$ & $0.71(0.53-0.94)$ & 0.02 & $0.77(0.53-1.13)$ & 0.19 & $0.63(0.41-0.96)$ & 0.02 \\
\hline rs $17017488^{(\mathrm{D})^{*}}$ & $5 \times 10^{-3}$ & $0.71(0.53-0.94)$ & 0.02 & $0.75(0.50-1.11)$ & 0.15 & $0.66(0.44-1.01)$ & 0.06 \\
\hline rs10511980 ${ }^{(\mathrm{D})^{*}}$ & $5 \times 10^{-3}$ & $0.69(0.52-0.90)$ & $8 \times 10^{-3}$ & $0.66(0.46-0.96)$ & 0.03 & $0.72(0.47-1.09)$ & 0.12 \\
\hline rs11172024 ${ }^{(\mathrm{D})^{*}}$ & $5 \times 10^{-3}$ & $0.66(0.46-0.93)$ & 0.02 & $0.61(0.37-1.00)$ & 0.05 & $0.71(0.42-1.17)$ & 0.18 \\
\hline rs6052937 $7^{(\mathrm{D})^{*}}$ & $5 \times 10^{-3}$ & $0.70(0.52-0.94)$ & 0.02 & $0.73(0.48-1.13)$ & 0.16 & $0.65(0.42-1.01)$ & 0.054 \\
\hline rs $10500248^{(\mathrm{D})^{* *}}$ & $5 \times 10^{-3}$ & $1.70(1.11-2.61)$ & 0.01 & $1.81(1.02-3.21)$ & 0.04 & $1.59(0.84-3.00)$ & 0.16 \\
\hline
\end{tabular}


Appendix Table 23 continued....

\begin{tabular}{|c|c|c|c|c|c|c|c|}
\hline & & Overall & & Male & & Female & \\
\hline SNP & $\begin{array}{c}\text { Log rank } \\
\text { P value }\end{array}$ & HR (95\% CI) & $P$ value & HR (95\% CI) & $P$ value & HR (95\% CI) & P value \\
\hline rs2124906 $6^{(\mathrm{D})^{* *}}$ & $5 \times 10^{-3}$ & $1.70(1.11-2.61)$ & 0.01 & $1.81(1.02-3.21)$ & 0.04 & $1.59(0.84-3.00)$ & 0.16 \\
\hline rs6572051 $1^{(\mathrm{D})^{* *}}$ & $5 \times 10^{-3}$ & $1.75(1.12-2.73)$ & 0.02 & $1.55(0.85-2.84)$ & 0.15 & $2.05(1.05-4.02)$ & 0.04 \\
\hline rs $17149645^{(\mathrm{D})^{* *}}$ & $5 \times 10^{-3}$ & $1.79(1.22-2.62)$ & $3 \times 10^{-3}$ & $2.03(1.13-3.66)$ & 0.02 & $1.68(1.01-2.77)$ & 0.05 \\
\hline rs6037033 ${ }^{(\mathrm{D})^{*}}$ & $6 \times 10^{-3}$ & $0.71(0.53-0.94)$ & 0.02 & $0.63(0.43-0.93)$ & 0.02 & $0.81(0.53-1.23)$ & 0.33 \\
\hline rs $1521244^{(\mathrm{D})^{*}}$ & $6 \times 10^{-3}$ & $1.41(1.06-1.88)$ & 0.02 & $1.18(0.81-1.72)$ & 0.4 & $1.75(1.14-2.70)$ & 0.01 \\
\hline rs1430838 ${ }^{(\mathrm{R})^{*}}$ & $6 \times 10^{-3}$ & $1.56(1.09-2.25)$ & 0.02 & $1.25(0.79-1.97)$ & 0.34 & $2.44(1.36-4.39)$ & 0.19 \\
\hline rs2116078 ${ }^{(\mathrm{R})^{*}}$ & $6 \times 10^{-3}$ & $1.56(1.09-2.25)$ & 0.02 & $1.25(0.79-1.97)$ & 0.34 & $2.44(1.36-4.39)$ & 0.19 \\
\hline rs $10157510^{(\mathrm{D})^{* *}}$ & $6 \times 10^{-3}$ & $1.72(1.10-2.71)$ & 0.02 & $1.61(0.90-2.88)$ & 0.11 & $1.93(0.95-3.92)$ & 0.07 \\
\hline rs $13116233^{(\mathrm{D})^{*}}$ & $7 \times 10^{-3}$ & $1.37(1.04-1.81)$ & 0.02 & $1.41(0.98-2.05)$ & 0.07 & $1.32(0.87-1.99)$ & 0.19 \\
\hline rs6519091 $^{(\mathrm{D})^{*}}$ & $7 \times 10^{-3}$ & $1.51(1.12-2.04)$ & $7 \times 10^{-3}$ & $1.35(0.91-2.00)$ & 0.13 & $1.76(1.10-2.83)$ & 0.02 \\
\hline rs2185230 ${ }^{(\mathrm{A})^{*}}$ & $7 \times 10^{-3}$ & $1.33(1.05-1.68)$ & 0.02 & $1.09(0.81-1.47)$ & 0.58 & $1.93(1.30-2.88)$ & $1 \times 10^{-3}$ \\
\hline rs7620914 ${ }^{(\mathrm{D})^{* *}}$ & $7 \times 10^{-3}$ & $1.50(1.06-2.11)$ & 0.02 & $1.84(1.16-2.90)$ & $9 \times 10^{-3}$ & $1.18(0.70-1.99)$ & 0.53 \\
\hline rs3850942 $2^{(\mathrm{D})^{*}}$ & $8 \times 10^{-3}$ & $0.62(0.40-0.95)$ & 0.03 & $0.65(0.35-1.22)$ & 0.18 & $0.59(0.33-1.07)$ & 0.08 \\
\hline rs3993401 ${ }^{(\mathrm{D})^{*}}$ & $8 \times 10^{-3}$ & $0.73(0.56-0.96)$ & 0.03 & $0.69(0.48-0.99)$ & 0.05 & $0.79(0.52-1.20)$ & 0.28 \\
\hline rs $13129052^{(\mathrm{D})^{* *}}$ & $8 \times 10^{-3}$ & $1.78(1.08-2.91)$ & 0.02 & $1.87(0.93-3.76)$ & 0.08 & $1.69(0.84-3.41)$ & 0.14 \\
\hline rs6093579 $9^{(\mathrm{D})^{* *}}$ & $8 \times 10^{-3}$ & $1.80(1.08-2.98)$ & 0.02 & $2.38(1.21-4.68)$ & 0.01 & $1.34(0.62-2.91)$ & 0.62 \\
\hline rs78323957 $7^{(\mathrm{D})^{* *}}$ & $8 \times 10^{-3}$ & $1.86(1.10-3.13)$ & 0.02 & $2.17(1.03-4.55)$ & 0.04 & $1.62(0.78-3.37)$ & 0.2 \\
\hline rs16950438 $8^{(\mathrm{D})^{* *}}$ & $8 \times 10^{-3}$ & $1.59(1.07-2.37)$ & 0.02 & $1.78(1.03-3.03)$ & 0.04 & $1.42(0.78-2.57)$ & 0.25 \\
\hline rs2125649 $9^{(\mathrm{D})^{*}}$ & $9 \times 10^{-3}$ & $0.69(0.50-0.96)$ & 0.03 & $0.70(0.44-1.10)$ & 0.12 & $0.69(0.43-1.10)$ & 0.12 \\
\hline rs5028798 ${ }^{(\mathrm{D})^{*}}$ & $9 \times 10^{-3}$ & $0.73(0.55-0.96)$ & 0.03 & $0.62(0.43-0.90)$ & 0.01 & $0.90(0.59-1.36)$ & 0.6 \\
\hline rs6151599 $9^{(\mathrm{D})^{* *}}$ & $9 \times 10^{-3}$ & $1.57(1.06-2.32)$ & 0.03 & $1.65(0.96-2.84)$ & 0.07 & $1.49(0.84-2.63)$ & 0.17 \\
\hline rs7492685 ${ }^{(\mathrm{D}) \dagger}$ & 0.01 & $1.72(1.07-2.77)$ & 0.03 & $1.54(0.49-4.89)$ & 0.46 & $1.76(1.04-2.99)$ & 0.04 \\
\hline
\end{tabular}


Appendix Table 23 continued....

\begin{tabular}{|c|c|c|c|c|c|c|c|}
\hline & & Overall & & Male & & Female & \\
\hline SNP & $\begin{array}{c}\text { Log rank } \\
\text { P value }\end{array}$ & HR $(95 \%$ CI $)$ & $P$ value & HR (95\% CI) & $P$ value & HR (95\% CI) & $P$ value \\
\hline rs13387046 $6^{(\mathrm{D}) \dagger \dagger}$ & 0.01 & $0.58(0.34-0.97)$ & 0.04 & $0.57(0.32-1.01)$ & 0.05 & $0.62(0.20-1.98)$ & 0.42 \\
\hline rs $4254428^{(\mathrm{D})^{*}}$ & 0.01 & $0.71(0.53-0.97)$ & 0.03 & $0.73(0.50-1.08)$ & 0.12 & $0.69(0.42-1.16)$ & 0.16 \\
\hline rs6510588 ${ }^{(\mathrm{D})^{*}}$ & 0.01 & $0.71(0.53-0.97)$ & 0.03 & $0.73(0.50-1.08)$ & 0.12 & $0.69(0.42-1.16)$ & 0.16 \\
\hline rs2051713 ${ }^{(\mathrm{D})^{*}}$ & 0.01 & $1.42(1.04-1.98)$ & 0.03 & $1.15(0.75-1.77)$ & 0.52 & $1.74(1.12-2.73)$ & 0.02 \\
\hline rs6109487 ${ }^{(\mathrm{D})^{*}}$ & 0.01 & $1.38(1.03-1.84)$ & 0.03 & $1.39(0.94-2.06)$ & 0.1 & $1.36(0.89-2.08)$ & 0.16 \\
\hline rs16979806 $6^{(\mathrm{R})^{*}}$ & 0.01 & $0.61(0.38-0.99)$ & 0.04 & $0.64(0.34-1.21)$ & 0.14 & $0.57(0.27-1.19)$ & 0.14 \\
\hline rs11120342 $2^{(\mathrm{D})^{*}}$ & 0.01 & $0.73(0.56-0.97)$ & 0.03 & $0.69(0.48-1.01)$ & 0.06 & $0.79(0.52-1.20)$ & 0.27 \\
\hline rs1481046 ${ }^{(\mathrm{D})^{*}}$ & 0.01 & $1.36(1.03-1.79)$ & 0.03 & $1.31(0.91-1.90)$ & 0.15 & $1.43(0.94-2.17)$ & 0.09 \\
\hline rs753712 ${ }^{(\mathrm{D})^{*}}$ & 0.01 & $0.72(0.54-0.97)$ & 0.03 & $0.70(0.47-1.04)$ & 0.07 & $0.75(0.48-1.18)$ & 0.17 \\
\hline rs6927353 ${ }^{(\mathrm{D})^{* *}}$ & 0.01 & $1.68(1.04-2.73)$ & 0.04 & $1.41(0.79-2.53)$ & 0.25 & $2.70(1.15-6.35)$ & 0.02 \\
\hline rs1800141 $1^{(\mathrm{D})^{* *}}$ & 0.01 & $1.76(1.04-2.98)$ & 0.04 & $1.26(0.65-2.43)$ & 0.49 & $4.65(1.91-11.32)$ & $1 \times 10^{-3}$ \\
\hline rs2389891 ${ }^{(\mathrm{D})^{* *}}$ & 0.01 & $1.57(1.05-2.37)$ & 0.03 & $1.92(1.14-3.26)$ & 0.02 & $1.20(0.62-2.33)$ & 0.59 \\
\hline rs $12188300^{(\mathrm{D})^{* *}}$ & 0.01 & $1.57(1.03-2.39)$ & 0.04 & $1.85(1.08-3.19)$ & 0.03 & $1.25(0.64-2.46)$ & 0.52 \\
\hline rs $7303641^{(\mathrm{D})^{* *}}$ & 0.01 & $1.60(1.06-2.42)$ & 0.03 & $1.84(1.06-3.19)$ & 0.03 & $1.35(0.72-2.55)$ & 0.35 \\
\hline rs34139105 $5^{(\mathrm{D})^{* *}}$ & 0.01 & $1.64(1.06-2.55)$ & 0.03 & $2.16(1.11-4.21)$ & 0.02 & $1.38(0.78-2.46)$ & 0.27 \\
\hline rs9328207 $7^{(\mathrm{D})^{* *}}$ & 0.01 & $1.70(1.06-2.73)$ & 0.03 & $1.57(0.86-2.88)$ & 0.15 & $1.94(0.91-4.15)$ & 0.09 \\
\hline rs $10514074^{(\mathrm{D})^{* *}}$ & 0.01 & $1.60(1.07-2.38)$ & 0.02 & $1.66(1.01-2.71)$ & 0.05 & $1.49(0.75-2.98)$ & 0.26 \\
\hline rs61744122 $2^{(\mathrm{D})^{* *}}$ & 0.01 & $1.68(1.05-2.69)$ & 0.03 & $1.63(0.82-3.27)$ & 0.17 & $1.72(0.90-3.28)$ & 0.10 \\
\hline rs $17170286^{(\mathrm{D})^{* *}}$ & 0.01 & $1.60(1.06-2.42)$ & 0.03 & $2.36(1.34-4.16)$ & $3 \times 10^{-3}$ & $1.12(0.61-2.06)$ & 0.72 \\
\hline rs7776264 ${ }^{(\mathrm{D}) \dagger}$ & 0.02 & $1.33(1.01-1.76)$ & 0.04 & $1.23(0.84-1.79)$ & 0.28 & $1.47(0.97-2.24)$ & 0.07 \\
\hline $\mathrm{rs} 468771^{(\mathrm{R}) \dagger}$ & 0.02 & $1.77(1.03-3.05)$ & 0.04 & $1.64(0.91-2.94)$ & 0.10 & $3.32(0.79-13.88)$ & 0.10 \\
\hline rs1713676 ${ }^{(\mathrm{D}) \dagger}$ & 0.02 & $1.56(1.03-2.37)$ & 0.04 & $1.68(1.03-2.74)$ & 0.04 & $1.29(0.56-2.96)$ & 0.55 \\
\hline
\end{tabular}


Appendix Table 23 continued....

\begin{tabular}{|c|c|c|c|c|c|c|c|}
\hline & & Overall & & Male & & Female & \\
\hline SNP & $\begin{array}{c}\text { Log rank } \\
\text { P value }\end{array}$ & HR $(95 \%$ CI $)$ & $P$ value & HR (95\% CI) & $P$ value & HR (95\% CI) & $P$ value \\
\hline rs349714 $4^{(\mathrm{R}) \dagger}$ & 0.02 & $0.70(0.50-0.98)$ & 0.04 & $0.68(0.40-1.14)$ & 0.15 & $0.72(0.47-1.11)$ & 0.14 \\
\hline rs2229528 $8^{(\mathrm{D}) \dagger \dagger}$ & 0.02 & $1.78(1.02-3.12)$ & 0.04 & $1.64(0.90-3.00)$ & 0.11 & $3.32(0.79-13.88)$ & 0.10 \\
\hline rs35693261 & 0.02 & $1.76(1.00-3.08)$ & 0.05 & $1.53(0.84-2.80)$ & 0.17 & $8.41(1.94-36.47)$ & $4 \times 10^{-3}$ \\
\hline rs368390 (D)† & 0.02 & $0.63(0.40-1.01)$ & 0.053 & $0.59(0.36-0.97)$ & 0.04 & $1.06(0.33-3.38)$ & 0.92 \\
\hline rs6936494 ${ }^{(\mathrm{D}) \dagger \dagger}$ & 0.02 & $0.66(0.44-1.00)$ & 0.053 & $0.66(0.42-1.04)$ & 0.07 & $0.69(0.24-1.95)$ & 0.48 \\
\hline rs9842465 & 0.02 & $1.75(1.00-3.06)$ & 0.05 & $1.75(0.95-3.21)$ & 0.07 & $1.77(0.43-7.26)$ & 0.43 \\
\hline rs665159 $9^{(\mathrm{D})^{*}}$ & 0.02 & $1.35(1.00-1.82)$ & 0.05 & $1.19(0.80-1.78)$ & 0.38 & $1.65(1.04-2.63)$ & 0.03 \\
\hline rs924615 ${ }^{(\mathrm{D})^{*}}$ & 0.02 & $0.75(0.57-0.99)$ & 0.04 & $0.73(0.50-1.06)$ & 0.10 & $0.78(0.52-1.19)$ & 0.25 \\
\hline rs877111 ${ }^{(\mathrm{D})^{*}}$ & 0.02 & $1.31(1.00-1.73)$ & 0.052 & $1.30(0.90-1.89)$ & 0.17 & $1.30(0.86-1.96)$ & 0.21 \\
\hline rs742390 $(\mathrm{D})^{*}$ & 0.02 & $1.33(1.01-1.75)$ & 0.04 & $1.36(0.94-1.96)$ & 0.10 & $1.31(0.87-1.98)$ & 0.20 \\
\hline rs2620870 ${ }^{(\mathrm{D})^{*}}$ & 0.02 & $1.35(1.01-1.82)$ & 0.05 & $1.40(0.94-2.08)$ & 0.10 & $1.28(0.82-2.01)$ & 0.28 \\
\hline rs1868368 ${ }^{(\mathrm{D})^{*}}$ & 0.02 & $0.76(0.58-1.01)$ & 0.054 & $0.67(0.46-0.97)$ & 0.04 & $0.89(0.59-1.34)$ & 0.58 \\
\hline rs762562 $2^{(\mathrm{D})^{*}}$ & 0.02 & $0.76(0.58-1.00)$ & 0.05 & $0.78(0.54-1.14)$ & 0.20 & $0.71(0.47-1.07)$ & 0.10 \\
\hline rs11171543 ${ }^{(\mathrm{D})^{*}}$ & 0.02 & $0.73(0.53-1.00)$ & 0.051 & $0.65(0.42-0.99)$ & 0.05 & $0.85(0.53-1.36)$ & 0.50 \\
\hline $\mathrm{rs} 36086854^{(\mathrm{D})^{* *}}$ & 0.02 & $1.65(1.02-2.68)$ & 0.04 & $1.54(0.86-2.78)$ & 0.15 & $1.92(0.82-4.48)$ & 0.13 \\
\hline rs $1835154^{(\mathrm{D})^{* *}}$ & 0.02 & $1.71(1.03-2.84)$ & 0.04 & $2.00(1.03-3.88)$ & 0.04 & $1.42(0.65-3.11)$ & 0.37 \\
\hline rs11175471 ${ }^{(\mathrm{D})^{* *}}$ & 0.02 & $1.50(1.01-2.22)$ & 0.04 & $1.44(0.86-2.41)$ & 0.16 & $1.59(0.86-2.94)$ & 0.14 \\
\hline rs $28421666^{(\mathrm{D})^{* *}}$ & 0.02 & $1.47(1.00-2.15)$ & 0.05 & $1.46(0.87-2.46)$ & 0.15 & $1.48(0.84-2.59)$ & 0.18 \\
\hline rs $17055478^{(\mathrm{D})^{* *}}$ & 0.02 & $1.58(1.01-2.48)$ & 0.05 & $1.97(1.01-3.84)$ & 0.05 & $1.35(0.73-2.48)$ & 0.34 \\
\hline rs3805114 ${ }^{(\mathrm{D})^{* *}}$ & 0.02 & $1.62(1.01-2.58)$ & 0.04 & $1.84(0.99-3.40)$ & 0.05 & $1.37(0.66-2.85)$ & 0.39 \\
\hline rs $17140942^{(\mathrm{D})^{* *}}$ & 0.02 & $1.65(1.02-2.66)$ & 0.04 & $1.46(0.76-2.82)$ & 0.26 & $1.92(0.95-3.85)$ & 0.07 \\
\hline rs6696657 $7^{(\mathrm{D}) \dagger}$ & 0.03 & $1.31(0.99-1.74)$ & 0.06 & $1.32(0.99-1.76)$ & 0.06 & $0.61(0.05-6.73)$ & 0.69 \\
\hline
\end{tabular}


Appendix Table 23 continued....

\begin{tabular}{|c|c|c|c|c|c|c|c|}
\hline & & Overall & & Male & & Female & \\
\hline SNP & $\begin{array}{c}\text { Log rank } \\
\text { P value }\end{array}$ & HR (95\% CI) & $P$ value & HR (95\% CI) & $P$ value & HR (95\% CI) & P value \\
\hline rs3857193 ${ }^{(\mathrm{D}) \dagger}$ & 0.03 & $0.76(0.56-1.01)$ & 0.06 & $0.82(0.56-1.20)$ & 0.30 & $0.68(0.44-1.06)$ & 0.09 \\
\hline rs117923468 $(\mathrm{D}) \dagger$ & 0.03 & $1.71(0.98-3.00)$ & 0.06 & $1.58(0.86-2.90)$ & 0.14 & $3.01(0.73-12.40)$ & 0.13 \\
\hline rs16973890 (D)† & 0.03 & $1.71(0.98-3.00)$ & 0.06 & $1.58(0.86-2.90)$ & 0.14 & $3.01(0.73-12.40)$ & 0.13 \\
\hline rs182319278 (D)† & 0.03 & $1.71(0.98-3.00)$ & 0.06 & $1.58(0.86-2.90)$ & 0.14 & $3.01(0.73-12.40)$ & 0.13 \\
\hline rs7818844 ${ }^{(\mathrm{D})^{*}}$ & 0.03 & $0.74(0.54-1.01)$ & 0.06 & $0.76(0.49-1.16)$ & 0.20 & $0.72(0.45-1.14)$ & 0.16 \\
\hline rs2899469 ${ }^{(\mathrm{D})^{*}}$ & 0.03 & $1.30(0.98-1.73)$ & 0.07 & $1.08(0.74-1.60)$ & 0.68 & $1.71(1.12-2.62)$ & 0.01 \\
\hline rs202050948 $(\mathrm{D})^{*}$ & 0.03 & $0.70(0.49-1.02)$ & 0.06 & $0.67(0.40-1.11)$ & 0.12 & $0.74(0.42-1.31)$ & 0.30 \\
\hline rs7330286 $6^{(\mathrm{D})^{* *}}$ & 0.03 & $1.55(0.97-2.48)$ & 0.07 & $1.18(0.61-2.28)$ & 0.61 & $1.66(0.98-2.82)$ & 0.06 \\
\hline rs11651675 & 0.03 & $1.69(1.00-2.87)$ & 0.05 & $1.41(0.57-3.46)$ & 0.46 & $1.89(0.98-3.65)$ & 0.06 \\
\hline rs $10109794^{(\mathrm{D})^{* *}}$ & 0.03 & $1.60(0.98-2.61)$ & 0.06 & $1.83(0.97-3.46)$ & 0.06 & $1.34(0.62-2.90)$ & 0.46 \\
\hline rs568760 ${ }^{(\mathrm{D})^{* *}}$ & 0.03 & $1.65(0.99-2.74)$ & 0.06 & $1.58(0.90-2.78)$ & 0.11 & $1.98(0.62-6.33)$ & 0.25 \\
\hline rs6851586 $6^{(\mathrm{D})^{* *}}$ & 0.03 & $1.49(0.97-2.29)$ & 0.07 & $1.06(0.60-1.86)$ & 0.85 & $2.93(1.51-5.71)$ & $2 \times 10^{-3}$ \\
\hline rs3790126 $6^{(\mathrm{D})^{* *}}$ & 0.03 & $1.59(1.00-2.53)$ & 0.05 & $2.15(1.07-4.31)$ & 0.03 & $1.30(0.71-2.41)$ & 0.40 \\
\hline rs41285557 $7^{(\mathrm{D})^{* *}}$ & 0.03 & $1.63(0.99-2.69)$ & 0.05 & $1.66(0.67-4.10)$ & 0.28 & $1.63(0.90-2.94)$ & 0.11 \\
\hline rs $17407084^{(\mathrm{D})^{* *}}$ & 0.03 & $1.58(0.98-2.56)$ & 0.06 & $1.43(0.72-2.83)$ & 0.31 & $1.77(0.90-3.47)$ & 0.10 \\
\hline rs7172316 ${ }^{(\mathrm{D})^{* *}}$ & 0.03 & $1.57(0.98-2.51)$ & 0.06 & $1.40(0.75-2.64)$ & 0.29 & $1.82(0.90-3.69)$ & 0.10 \\
\hline rs10902652 $2^{(\mathrm{D})^{* *}}$ & 0.03 & $1.58(0.98-2.56)$ & 0.06 & $1.27(0.64-2.51)$ & 0.50 & $2.07(1.04-4.13)$ & 0.04 \\
\hline rs8013602 $2^{(\mathrm{D})^{* *}}$ & 0.03 & $1.57(0.97-2.52)$ & 0.06 & $1.29(0.67-2.47)$ & 0.45 & $2.06(1.03-4.13)$ & 0.04 \\
\hline rs4680582 $2^{(\mathrm{D}) \dagger}$ & 0.04 & $1.30(0.97-1.75)$ & 0.08 & $1.34(0.88-2.04)$ & 0.17 & $1.26(0.83-1.91)$ & 0.28 \\
\hline rs4792149 & 0.04 & $1.33(0.96-1.84)$ & 0.09 & $1.35(0.92-1.99)$ & 0.13 & $1.27(0.69-2.34)$ & 0.44 \\
\hline rs2874641 ${ }^{(\mathrm{D}) \dagger}$ & 0.04 & $1.30(0.98-1.72)$ & 0.07 & $1.24(0.84-1.83)$ & 0.11 & $1.36(0.90-2.08)$ & 0.15 \\
\hline rs1550017 ${ }^{(\mathrm{A}) \dagger}$ & 0.04 & $1.36(0.99-1.88)$ & 0.06 & $1.36(0.95-1.95)$ & 0.10 & $1.38(0.69-2.77)$ & 0.36 \\
\hline
\end{tabular}


Appendix Table 23 continued....

\begin{tabular}{|c|c|c|c|c|c|c|c|}
\hline & & Overall & & Male & & Female & \\
\hline SNP & $\begin{array}{c}\text { Log rank } \\
\text { P value }\end{array}$ & HR (95\% CI) & P value & HR (95\% CI) & P value & HR $(95 \%$ CI) & $P$ value \\
\hline rs34022679 (D)† & 0.04 & $1.58(0.96-2.59)$ & 0.07 & $1.77(1.03-3.03)$ & 0.04 & $0.90(0.22-3.66)$ & 0.88 \\
\hline rs2031293 $3^{(\mathrm{D})^{*}}$ & 0.04 & $0.76(0.56-1.03)$ & 0.08 & $0.99(0.65-1.53)$ & 0.99 & $0.48(0.30-0.76)$ & $2 \times 10^{-3}$ \\
\hline rs7016929 $9^{(\mathrm{D})^{*}}$ & 0.04 & $0.78(0.59-1.03)$ & 0.08 & $0.84(0.63-1.13)$ & 0.25 & $0.81(0.56-1.17)$ & 0.27 \\
\hline rs $1004327^{(\mathrm{A})^{*}}$ & 0.04 & $1.25(1.01-1.26)$ & 0.04 & $1.19(0.88-1.60)$ & 0.25 & $1.34(0.97-1.85)$ & 0.08 \\
\hline rs37762 $1^{\circledR *}$ & 0.04 & $0.68(0.44-1.05)$ & 0.08 & $0.76(0.44-1.31)$ & 0.32 & $0.57(0.27-1.19)$ & 0.14 \\
\hline rs7304215 ${ }^{(\mathrm{D})^{* *}}$ & 0.04 & $1.53(0.97-2.44)$ & 0.07 & $2.00(1.03-3.88)$ & 0.04 & $1.25(0.66-2.36)$ & 0.50 \\
\hline rs $17039265^{(\mathrm{D})^{* *}}$ & 0.04 & $1.58(0.95-2.63)$ & 0.08 & $1.39(0.74-2.62)$ & 0.31 & $2.08(0.89-4.84)$ & 0.09 \\
\hline rs9728577 ${ }^{(\mathrm{D})^{* *}}$ & 0.04 & $1.58(0.95-2.63)$ & 0.08 & $1.39(0.74-2.62)$ & 0.31 & $2.08(0.89-4.84)$ & 0.09 \\
\hline rs $75411676^{(\mathrm{D})^{* *}}$ & 0.04 & $1.58(0.95-2.63)$ & 0.08 & $1.39(0.74-2.62)$ & 0.31 & $2.08(0.89-4.84)$ & 0.09 \\
\hline rs8176018 ${ }^{(\mathrm{D})^{* * *}}$ & 0.04 & $1.62(0.96-2.72)$ & 0.07 & $0.94(0.38-2.30)$ & 0.89 & $2.51(1.29-4.86)$ & $7 \times 10^{-3}$ \\
\hline rs9407838 $8^{(\mathrm{D})^{* *}}$ & 0.04 & $1.41(0.96-2.08)$ & 0.08 & $1.35(0.81-2.24)$ & 0.25 & $1.51(0.84-2.73)$ & 0.17 \\
\hline rs16964079 $9^{(\mathrm{D})^{* *}}$ & 0.04 & $1.48(0.94-2.32)$ & 0.09 & $1.50(0.87-2.60)$ & 0.15 & $1.44(0.66-3.14)$ & 0.36 \\
\hline rs3130161 ${ }^{(\mathrm{D})^{* *}}$ & 0.04 & $1.52(0.86-2.41)$ & 0.07 & $2.06(1.11-3.82)$ & 0.02 & $1.13(0.56-2.25)$ & 0.73 \\
\hline rs $12257453^{(\mathrm{D})^{* *}}$ & 0.04 & $1.53(0.97-2.43)$ & 0.07 & $1.55(0.86-2.81)$ & 0.15 & $1.51(0.73-3.14)$ & 0.27 \\
\hline rs11833839 $9^{(\mathrm{D})^{* *}}$ & 0.04 & $1.51(0.94-2.42)$ & 0.09 & $1.71(0.89-3.30)$ & 0.11 & $1.34(0.68-2.64)$ & 0.40 \\
\hline rs6904762 $2^{\mathbb{Q} \dagger}$ & 0.05 & $0.70(0.46-1.07)$ & 0.10 & $0.54(0.26-1.11)$ & 0.10 & $0.83(0.49-1.41)$ & 0.48 \\
\hline $\mathrm{rs} 26784^{(\mathrm{A})^{*}}$ & 0.05 & $1.23(1.00-1.50)$ & 0.05 & $1.26(0.93-1.70)$ & 0.14 & $1.20(0.91-1.59)$ & 0.19 \\
\hline rs3204798 ${ }^{(\mathrm{D})^{* *}}$ & 0.05 & $1.57(0.94-2.62)$ & 0.08 & $1.39(0.74-2.62)$ & 0.31 & $2.03(0.87-4.74)$ & 0.10 \\
\hline rs3729598 ${ }^{(\mathrm{D})^{* *}}$ & 0.05 & $1.58(0.94-2.66)$ & 0.08 & $1.26(0.64-2.51)$ & 0.51 & $2.31(1.05-5.13)$ & 0.04 \\
\hline rs35384751 $1^{(\mathrm{D})^{* *}}$ & 0.05 & $1.58(0.94-2.65)$ & 0.08 & $1.99(0.96-4.13)$ & 0.07 & $1.31(0.63-2.71)$ & 0.47 \\
\hline
\end{tabular}


Appendix Table 23 continued...

\begin{tabular}{|c|c|c|c|c|c|c|c|}
\hline & & Overall & & Male & & Female & \\
\hline SNP & $\begin{array}{c}\text { Log rank } \\
\text { P value }\end{array}$ & HR $(95 \%$ CI) & $P$ value & HR $(95 \%$ CI $)$ & $P$ value & HR $(95 \%$ CI) & $P$ value \\
\hline rs11234604 ${ }^{(\mathrm{D})^{* *}}$ & 0.05 & $1.51(0.94-2.40)$ & 0.09 & $1.39(0.74-2.60)$ & 0.31 & $1.68(0.84-3.36)$ & 0.15 \\
\hline rs $2864680^{(\mathrm{D})^{* * *}}$ & 0.05 & $1.53(1.03-2.28)$ & 0.03 & $1.42(0.84-2.40)$ & 0.19 & $1.71(0.95-3.09)$ & 0.08 \\
\hline
\end{tabular}

(D): Dominant genetic model; (A): Additive genetic model; (R): Recessive genetic model

*SNPs yielded age-specific associations results based on the P values; ** SNPs yielded age-specific associations results based on the effect sizes

${ }^{\dagger}$ SNPs yielded gender-specific associations results based on the P values; ${ }^{\dagger}$ SNPs yielded gender-specific associations results based on the effect sizes

$\mathrm{SNP}=$ single nucleotide polymorphism $; \mathrm{HR}=$ hazard ratio $; \mathrm{CI}=$ confidence interval 
Appendix Table 24. Previously identified gender-specific genetic risk markers in childhood ALL and their evidence in the present study

\begin{tabular}{|c|c|c|c|c|}
\hline SNP & Gene & $\begin{array}{c}\text { Previous association } \\
\text { (P interaction) }\end{array}$ & $\begin{array}{c}\text { P value* }^{*} \\
\text { (present study) }\end{array}$ \\
\hline rs10994982 & ARID5B & 0.01 & Male-specific & Male-specific \\
\hline rs10740055 & ARID5B & 0.03 & association differs in direction & 0.91 \\
\hline rs2395185 & $H L A-D R B 9$ & 0.001 & association differs in direction & 0.58 \\
\hline rs7192 & HLA-DRA & 0.03 & association differs in direction & 0.06 \\
\hline rs419788 & SKIV2L & 0.04 & association differs in direction & 0.08 \\
\hline rs224589 & $S L C 11 A 2$ & 0.02 & association differs in direction & 0.47 \\
\hline rs3130342 & TNXB & 0.04 & association differs in direction \\
\hline rs422982 & NRAMP2 & 0.007 & association differs in direction & 0.92 \\
\hline rs807212 & HFE & 0.05 & 0.46 \\
\hline
\end{tabular}

$\mathrm{ALL}=$ acute lymphoblastic leukemia; $\mathrm{SNP}=$ single nucleotide polymorphism

*P value represents statistical interaction 


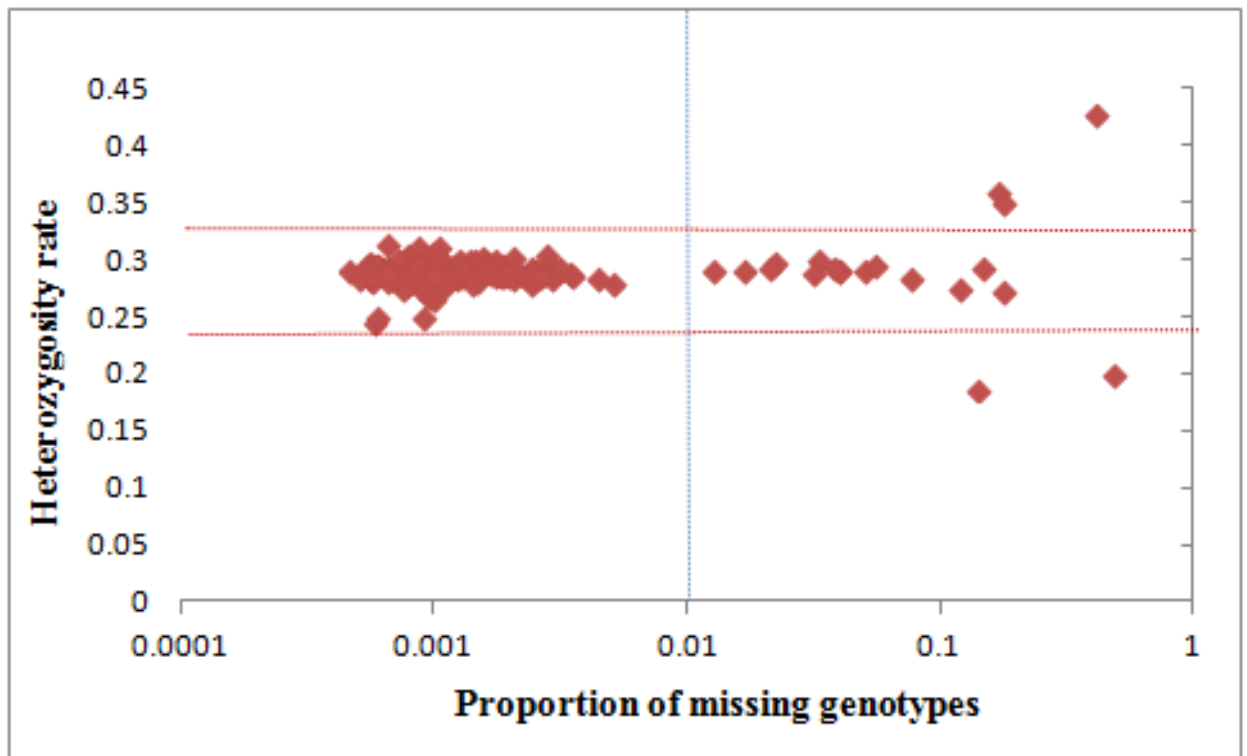

Appendix Figure 1. Genotype failure rate versus heterozygosity rate across all individuals in the study. Shading indicates sample density and dashed line denote quality control threshold. X-axis represents proportion of missing genotypes on log scale and $\mathrm{Y}$-axis represents heterozygosity rates. 
VITA

\section{SANDEEP KUMAR SINGH}

Born, Pratapgarh, Uttar Pradesh, India

2010 - present

Ph.D. Candidate in Public Health (Environmental and

Occupational Health)

Florida International University

Miami, Florida

Jan 2014- Aug 2014

Graduate Assistant

Robert Stempel College of Public Health and Social Work

Florida International University

Aug 2013 - Dec 2013

Graduate Assistant

Department of Epidemiology

Robert Stempel College of Public Health and Social Work

Aug 2010 - Aug 2012

Graduate Assistant

Robert Stempel College of Public Health and Social Work Department of Environmental and Occupational Health

$2007-2009$

MPH, Environmental Health

Western Kentucky University

Bowling Green, Kentucky

\section{PUBLICATIONS AND PRESENTATIONS (ABRIDGED LIST)}

Singh SK, Lupo PJ, Scheurer ME, Saxena A, Kennedy AE, Ibrahimou B, McCauley JL, Okcu M, Dorak MT. Genome-wide association study of childhood acute lymphoblastic leukaemia risk with gender-specificity. Accepted for poster presentation at the European Human Genetics Conference 2015 Glasgow, Scotland, United Kingdom June 6-9, 2015

Singh SK, Lupo PJ, Scheurer ME, Kennedy AE, Okcu MF, Dorak MT. HLA region contributes to the gender differential in childhood leukaemia risk. Accepted for poster presentation at the 29th European Immunogenetics and Histocompatibility Conference, Geneva, Switzerland, April 26-29, 2015

Kekik C, Singh SK, Yavuz C, Oguz FS, Dorak MT. HLA and childhood acute myeloid leukemia. Accepted for poster presentation at the 29th European Immunogenetics and Histocompatibility Conference, Geneva, Switzerland, April 26-29, 2015 
Singh SK. Re: Association of polymorphism in cytochrome P450 2C9 with susceptibility to head and neck cancer and treatment outcome; Pragmatic use of Hardy-Weinberg equilibrium and statistical interaction analysis. App. \& Trans. Gen 2014. 3: 48-49

Kennedy AE, Singh SK, Dorak MT. Re: Genome-wide association study of classical Hodgkin lymphoma and Epstein - Barr virus status-defined subgroups (Scientific Correspondence). Journal of the National Cancer Institute 2012. 104 (11): 884-885

Singh SK, Dorak MT. Alternative Explanations for HLA and Disease Associations. Oral presentation at the $28^{\text {th }}$ European Immunogenetics and Histocompatibility Conference, Stockholm, Sweden, June 25-28, 2014. Tissue Antigens 2014;84(1):9

Oguz FS, Kekik C, Singh SK, Saribeyoglu E, Anak S, Ozturk G, Dorak MT. HLA and age-of-onset in childhood acute lymphoblastic leukaemia. Poster presentation at the $28^{\text {th }}$ European Immunogenetics and Histocompatibility Conference, Stockholm, Sweden, June 25-28, 2014. Tissue Antigens 2014;84(1):146

$\underline{\text { Singh SK}}$, Ben Taleb Z, Kennedy AE, Dorak MT. X-Linked Immune Regulatory Genes Polymorphisms, Childhood Acute Lymphoblastic Leukemia Risk and Male Disadvantage. Presented at the 14th Annual Biomedical \& Comparative Immunology Symposium, Florida International University, Miami, FL, Feb 13-14, 2014.

Singh SK, Ben Taleb Z, Kennedy AE, Villalba K, Dorak MT. Further exploration of $H L A$ region associations with lung cancer risk. Presented at the 39th Annual Meeting of the American Society for Histocompatibility and Immunogenetics. Chicago, IL, Nov 1721, 2013.

Kennedy AK, Singh SK, Villalba K, Dorak MT. Analysis of HLA region polymorphism associated with cancer. Presented at the 39th Annual Meeting of the American Society for Histocompatibility and Immunogenetics. Chicago, IL, Nov 17-21, 2013.

Singh SK, Kennedy AE, Dorak MT. DDX3X gene polymorphism and childhood acute lymphoblastic leukemia risk. Presented at the 15th Annual Biomedical \& Comparative Immunology Symposium, Florida International University, Miami, FL, March 7th - 8th, 2013.

Singh SK, Saxena A, Das S, Dorak MT. NOTCH4 polymorphisms, functional assessment and associations with breast cancer susceptibility. Presented at the 38th Annual Meeting of the American Society for Histocompatibility and Immunogenetics. San Juan, PR, Oct 8-12, 2012.

Kennedy AE, Singh SK, Scheurer ME, Okcu MF, Dorak MT. Association of HLA-linked lymphoma risk markers with childhood lymphoblastic leukemia. Presented at the 38th Annual Meeting of the American Society for Histocompatibility and Immunogenetics,. San Juan, PR, Oct 8-12, 2012. 